\begin{abstract}
Title of dissertation: Leveraged Lévy Processes as Models for Stock Prices

Yue Xiao, Doctor of Philosophy, 2005

\section{Dissertation directed by: Professor Dilip B. Madan Robert H. Smith School of Business}

We entertain the hypothesis that leverage considerations are relevant in describing the evolution of asset returns both statistically and risk neutrally. Adopting a constant elasticity of variance formulation in the context of a general Lévy process as the driving uncertainty we show that the presence of leverage effects in this form has the implication that asset pricing satisfy a scaling hypothesis. Examples of continuous and pure jump Lévy cases are constructed and explicit forms for the semigroups are obtained with empirical investigations.

In our study, we build in the leverage effect by introducing a time change dependent on the level of asset and hence affect the expected local volatility in an explicit manner. This is a fairly direct approach in the context of Lévy processes. The continuous case in our study coincides with the development of the constant elasticity of variance models. We however, conduct our investigation in the continuous case through our incorporation of BESQ process as the semi-stable Markov process. In the pure jump case with underlying time changed Lévy process being specified as CGMY process, we hope to engage the leverage effect as well as the 
ability of explaining long-tailedness and skewness as already being provided by using such pure jump Lévy process with infinite activity. We show how to implement Generalized Method of Moments in this case to estimate parameters without the assumption of knowing the law of the process.

The development of forward Partial Integro-Differential Equations is under a general setup and shows great advantage over the backward ones. In both the continuous case and the pure jump case, we show how to calibrate our model parameters by solving such forward PIDEs and compare model prices to the market data. Although the numerical approach used in the pure jump case is discussed in the context of CGMY process, it is evident that the approach can be extended to a general frame work indifferent of the choice of Lévy process and shall be similarly carried out where other Lévy processes are specified in our model. 


\title{
Leveraged Lévy Processes as Models for Stock Prices
}

\author{
by
}

Yue Xiao

Dissertation submitted to the Faculty of the Graduate School of the University of Maryland, College Park in partial fulfillment of the requirements for the degree of Doctor of Philosophy 2005

Advisory Commmittee:

Professor Dilip B. Madan, Chair/Advisor

Professor David Levermore

Professor Michael Fu

Professor Konstantina Trivisa

Professor Nagpurnanand Prabhala 
(c) Copyright by

Yue Xiao

2005 


\section{ACKNOWLEDGMENTS}

First I would like to thank my parents for always being so supportive of all my endeavors. No matter how far I am from home and no matter how much or little I achieve, they have always given me endless encouragement, comfort, and most importantly, unconditional love and support. The weekly phone calls with my parents refreshes my whole week of work. Telling them what I do and summarizing my progress each week surprisingly detangles my thoughts. Their advice to me, though not always necessarily useful, keeps me motived and makes me aware of how much I am loved by them. I would like to dedicate my dissertation especially to my mother as she has always been so concerned with my study and for her great effort of listening to my progress, and even trying to understand the subject that I am focusing on. Without the help of my mother and the opportunity of education she has presented to me, I would never have had the chance to come here to pursue my goals. Her understanding of my situation and support of my abnormal two Ph.D. ambition always surprises me and sometimes even outbraves my own.

I would also like to give thanks to my fiancee Aaron, as being the closest person in my life to share with me my experience of research as well as my experience of life. Besides the emotional support that has kept me going through the often frustrating time of graduate life, Aaron has also given me repeated help with $\mathrm{LT}_{\mathrm{E}} \mathrm{X}$, computers and much much more. Without his help and being in my life, I would not have 
done this so quickly. The numerous conversations we had in the situation of my frustration always renewed my confidence to continue working towards my goal and gave me new perception of academic research.

I also thank all of my friends at the University of Maryland, both from the Department of Mathematics and from the School of Business for helping me from time to time on either research or personal needs, and keeping me entertained in my spare time. I feel so lucky that I have met so many good friends, this has made graduate life so much more exciting and beautiful. I would like to give special thanks to Andy Kebo for patiently discussing my research and many other issues of school and life. I am also grateful for the many research discussions with my colleagues and friends Qin Xia, Bing Zhang, and Xianfang Wu.

I would like to thank all of my committee members, Dr. Dilip B. Madan, Dr. David Levermore, Dr. Michael Fu, Dr. Konstantina Trivisa and Dr. Nagpurnanand Prabhala, to be so kind to me and so helpful in my research. I greatly appreciate the help and patience that Dr. Levermore has given me, as well as his wise advice on many aspects of research and school life. I thank Dr. Fu for advising me on my Master's degree in Applied Math and on other research related questions that I had throughout graduate school. Again, I would like to thank Dr. Prabhala for being in my committee and coming all the way from Princeton to attend my defense.

Last but not least, I would like to thank my advisor, Dr. Dilip B. Madan, for his kindness, encouragement, repeated help and advice, and for sharing his broad knowledge of mathematical finance. Without his effort and being a good advisor, this dissertation would not have been finished so soon. His broad knowledge gave me 
good direction of areas suitable for me to work on and made my research possible. Through his step by step progress plan, it was easy for me to follow and concentrate on one issue at a time. His insights allowed us to approach problems at many different angles and resulted in this thesis. I also thank him for his advice and suggestions on my choice of career and future goals. His example of achieving two Ph.D. degrees has led to my ultimate ambition. 


\section{TABLE OF CONTENTS}

List of Figures vii

1 INTRODUCTION 1

2 LÉVY PROCESSES IN FINANCE 11

2.1 The Black-Scholes Model . . . . . . . . . . . . . . . . . 12

2.1.1 Stochastic Integrals and SDEs . . . . . . . . . . . . . . 12

2.1.2 Geometric Brownian Motion . . . . . . . . . . . . . . 13

2.1.3 The Black-Scholes Option Pricing Model . . . . . . . . . . . . 14

2.2 Lévy Processes . . . . . . . . . . . . . . . . . . . . . . 20

2.2.1 Introduction . . . . . . . . . . . . . . . . 20

2.2.2 Definitions and Theorems ................ 21

2.2.3 The CGMY Process . . . . . . . . . . . . . . . . . 26

2.3 Semigroups and Their Generators . . . . . . . . . . . . . . 28

3 SEMI-STABLE MARKOV PROCESSES 34

3.1 Semi-Stable Stochastic Processes . . . . . . . . . . . . . . . . 35

3.1.1 Definitions and Theorems .............. 35

3.1.2 Examples of Semi-Stable Processes . . . . . . . . . . . . . . . 40

3.2 Semi-Stable Markov Processes . . . . . . . . . . . . . . . . . . . . . 42

3.2.1 General Preliminaries . . . . . . . . . . . . . . . . . . 42

3.2.2 The Theorem . . . . . . . . . . . . . . . . . 46

3.2.3 The Diffusion Case . . . . . . . . . . . . . . . . . . . . . . . 48

3.2.4 The generator of a SSMP . . . . . . . . . . . . 51

3.2.5 Bessel Squared Processes . . . . . . . . . . . . . . . 53

3.3 Exponential Functionals of Brownian Motion and Related Processes . 59

4 LEVERAGING LÉVY VIA LAMPERTI 62

4.1 Leveraging Lévy . . . . . . . . . . . . . . . . . . . . . . . . . . . . . . . . . . . . . . . . . . 62

4.2 The General Model . . . . . . . . . . . . . . . . . 63

4.2.1 Leveraging Lévy . . . . . . . . . . . . . . . . . . 63

4.2.2 Lamperti and Leveraged Lévy . . . . . . . . . . . . . . . . . . 65

4.3 Stock Price Modeling Using Leveraged Lévy Processes . . . . . . . . 68

4.3.1 Martingale Leveraged Lévy Models . . . . . . . . . . . . . . . 69

4.3.2 Normalized Leveraged Lévy Models . . . . . . . . . . . . . . . 70

4.3.3 The Absolute Value of a Cauchy . . . . . . . . . . . . . . . 71

4.3.4 Exponential Tilting of a Lévy Process in General . . . . . . . 75

5 THE DIFFUSION CASE WITH SQUARED BESSEL 78

5.1 The Formulation . . . . . . . . . . . . . . . . . . . 78

5.2 Take Bessel Squared Process as the Lamperti . . . . . . . . . . . . . 81

5.3 Maximum Likelihood Estimation of Parameters . . . . . . . . . . . . 82

5.3.1 Data and Results . . . . . . . . . . . . . . . . . . 82 
5.4 Forward PDE in The Diffusion Case . . . . . . . . . . . . . . . 92

5.4 .1 Discretization . . . . . . . . . . . . . . . . . 95

5.4.2 Risk-Neutral Estimation by Solving PDE . . . . . . . . . . 97

6 THE DISCRETE CASE 102

6.1 The Model . . . . . . . . . . . . . . . . . . . . . . . . . . . 102

6.2 Deriving the Infinitesimal Generator for $S_{t} \ldots \ldots \ldots \ldots$. . . . 105

6.3 GMM Estimation . . . . . . . . . . . . . . . . . . . . . 108

6.3.1 Introduction of GMM . . . . . . . . . . . . . . . . 109

6.3.2 Using GMM in CGMY case . . . . . . . . . . . . . . . 116

6.3 .3 Estimation Results . . . . . . . . . . . . . . . . . . . . . 119

6.4 Backward Partial Integro-Differential Equations for Option Prices . . 127

6.5 Forward Partial Integro-Differential Equations . . . . . . . . . . . . 129

6.6 Using CGMY process as the underlying Lévy . . . . . . . . . . . . 136

6.6.1 Backward PIDE . . . . . . . . . . . . . . . . . . . . 138

6.6 .2 Forward PIDE . . . . . . . . . . . . . . . . . . . . . . 139

6.6 .3 Discretization . . . . . . . . . . . . . . . . . . . 145

6.6.4 The Evaluation of $f_{1}(a)$ and $f_{2}(a) \ldots \ldots \ldots \ldots$

6.6.5 The System . . . . . . . . . . . . . . . . . . . . 154

6.6.6 An Alternative Scheme for Faster Computations . . . . . . . . 160

6.6.7 Numerical Experiments . . . . . . . . . . . . . . . . . . . 163

7 CONCLUSION 167

$\begin{array}{lll}\text { A Evaluation of } \gamma+\omega & 170\end{array}$

B Integral Evaluation in the CGMY Case 175

B.0.8 Evaluation of Integral (6.41), when $\hat{\beta} \neq 1 \ldots \ldots . \ldots 175$

B.0.9 Evaluation of Integral (6.42), when $\hat{\beta} \neq 1 \ldots \ldots$

B.0.10 Evaluation of Integral (6.43), when $\hat{\beta} \neq 1 \ldots \ldots . \ldots 182$

B.0.11 Evaluation of Integral (6.44), when $\hat{\beta} \neq 1 \ldots \ldots . \ldots . . .184$

B.0.12 Evaluation of Integrals (6.41-6.44) when $\hat{\beta}=1 \ldots \ldots$. . . 187

Bibliography

193 


\section{LIST OF FIGURES}

2.1 One Sample Path of Geometric Brownian Motion . . . . . . . . . . . 15

5.1 S\&P500 Daily Prices from 09/21/1983 to 09/20/2004 . . . . . . . . 85

5.2 Data Fitting For Calls of All Strikes and Maturities on 12-31-2003.

Strikes vs. Option prices, $\star$ - Model prices, o - Market prices . . . . . 100

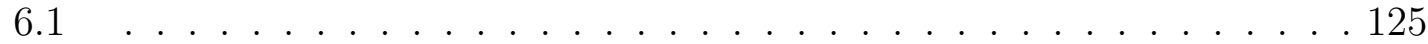

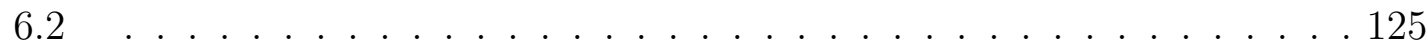

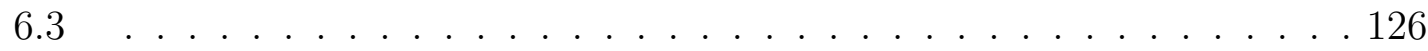

6.4 Calculated values of $f$ versus $\nu$ using FFT in Matlab with $G=10$, $M=10$ and $Y=0.5 \ldots \ldots . \ldots . \ldots . \ldots 154$

6.5 Data Fitting For Calls of All Strikes and Maturities on 12-31-2003.

Strikes vs. Option prices, $\star$ - Model prices, o - Market prices . . . . . 166 


\section{Chapter 1}

\section{INTRODUCTION}

The objective of this thesis is to enhance the applicability of Lévy processes as models for stock prices. We entertain the hypothesis that leverage considerations are relevant in describing the evolution of asset returns both statistically and risk neutrally. Adopting a constant elasticity of variance formulation in the context of a general Lévy process as the driving uncertainty we show that the presence of leverage effects in this form has the implication that asset pricing satisfy a scaling hypothesis. Examples of continuous and pure jump Lévy cases are constructed and explicit forms for the semigroups are obtained with empirical investigations.

Lévy processes have long been used in mathematical finance. In fact, the best known of all Lévy processes, Brownian motion, was originally introduced as a stock price model (see Bachelier (1900, [4]).) Osborne (1959, [54]) refined Bachelier's model by proposing the $\operatorname{exponential~} \exp \left(B_{t}\right)$ of Brownian motion as a stock price model. In a more systematic manner, the same process $\exp \left(B_{t}\right)$, which is called exponential, or geometric Brownian motion, was introduced as a stock price model by Samuelson (1965, [59]). Eight years later, in 1973, Black, Scholes and Merton demonstrated how to price European options based on the geometric Brownian model. This stock-price model is now called the Black-Scholes model, for which Scholes and Merton received the Nobel Prize for Economics in 1997 (Black had 
already died).

Despite of the success of Black-Scholes model, however, the analysis of univariate time series data on financial market asset returns has long confirmed their long-tailedness relative to the normal distribution. Analysis of such returns using data on option prices confirms this phenomenon on a risk neutral basis, with the addition now of a marked negative skewness as well. These observations have led to an increased adoption of alternative processes for describing these returns. Many researchers have begun to employ purely discontinuous Lévy processes with infinite activity (i.e. with infinitely many jumps in any interval of time). Lévy processes are now increasingly employed in both the analysis of value at risk and the pricing of options.

A Lévy process $X=(X(t), t \geq 0)$ is a right continuous process with independent stationary increments and can be decomposed as the sum of a linear drift, a Brownian motion and a pure jump process: $X(t)=\mu t+\sigma W(t)+\sum_{0 \leq u \leq t} \Delta Y(u)$. The jump process $Y=(Y(t), t \geq 0)$ is independent of $W(t)$ and the rate at which jumps of size $d z$ occur is governed by a positive measure $\nu(d z)$ which summarizes the distribution as well as the arrival intensity of jumps. The only restriction on $\nu(d z)$ is that $\int_{\mathbb{R}^{d} \backslash\{0\}} \frac{|z|^{2}}{1+|z|^{2}} \nu(d z)<\infty$ which ensures that the resulting process is a semimartingale. The jumps allowed under the Lévy process definition are slightly more general than Poisson jumps since the arrival rate can be infinite when $\int \nu(d z)=\infty$, but not much more so since such jumps can always be obtained as the uniform limit of Poisson jumps. A Lévy process is thus uniquely determined by its drift, its volatility and the measure $\nu(d z)$. The triple $(\mu, \sigma, \nu(d z))$ is often referred to as 
the semimartingale characteristics of $X(t)$ and Lévy models can conveniently be parameterized in terms of these quantities. Chapter 2 will focus on Lévy processes in Finance in more detail.

A classical result, due to John Lamperti, establishes a one-to-one correspondence between a class of strictly positive Markov processes that are self-similar or semi-stable (according to John Lamperti), and the class of one-dimensional Lévy processes. This correspondence is obtained by suitably time-changing the exponential of the Lévy process. In Chapter 3 Lamperti's 1962 and 1972 papers ([44], [45]) are studied, and we introduce the definitions and properties of the class of semistable Markov processes. Some important theorems and proofs are summarized as well.

The objective of this thesis is to enhance the applicability of Lévy processes by addressing some shortcomings associated with these processes. The particular issue we address here is that of the presence of leverage effects. Many researchers have documented a negative relationship between market volatilities and the level of asset prices. It is argued that this negative relation reflects greater risk taking by management, induced by a fall in the asset price, with a view of maximizing the option value of equity shareholders. This leverage effect is modeled in the context of diffusion by allowing the volatility to be a deterministic function of the spot price and has led to the development of the constant elasticity of variance models and the local volatility models. The analogs in the context of Lévy processes are absent. Stochastic volatility models in the context of Lévy processes have introduced correlation terms to capture this leverage effect but such an approach is relatively 
indirect. Recently, Carr, Geman, Madan and Yor (2003) have extended the local volatility models to allow for local Lévy models. In this thesis we address leverage directly in the context of a Lévy process.

In Chapter 4, We construct the Leveraged Lévy model for stock prices. We allow for a direct dependence of volatility for a Lévy process, measured by the speed at which the Lévy process is locally proceeding, on the price of asset and seek to determine probability laws of such a leveraged process. We show that such processes are closely linked to processes introduced by Lamperti $([45])$, and we term them the Lamperti processes associated with Lévy processes. We discover the probability laws and characteristics of the associated Lamperti process from those of the Lévy process and construct the Leveraged Lévy process as precisely the associated Lamperti process. This precise association explains our title.

We anticipate that the use of such leveraged Lévy processes will shed better light on the exact role of leverage in financial markets both statistically and risk neutrally. Our reasoning is that since Lévy processes can internally explain both long-tailedness and skewness without the addition of leverage, the estimation of a significant leverage effect is probably just that and not a proxy for other well known and stylized features of the return density. In the context of diffusions, given their documented inability to address these aspects of the density it is possible that estimated risk neutral leverage for example is just a reflection of well documented skewness and has little to do with volatilities actually moving with a market drop.

The development of the models here will also enable an assessment of the impact of leverage on the valuation of claims otherwise analyzed in a zero leverage 
context. We expect that subsequent developments will allow for both stochastic volatility and leverage by incorporating stochastic volatility into Lamperti processes.

In this dissertation we shall make the assumption that the process of interest, $S=(S(t), t \geq 0)$, is of Lévy type rather than literally being a Lévy process. This means that the characteristics $(\mu, \sigma, \nu)$ are allowed to be state dependent, which is of key importance for capturing essential features of financial data. Time homogeneous diffusions as well as processes with state dependent jump intensities and jump distributions are special cases. This expands the set of models to include most popular time homogeneous strongly Markovian semimartingales. As in the Lévy case, these processes can conveniently be parameterized by parameterizing the triplet of semimartingale characteristics which now uniquely define the process in terms of its conditional drift, its conditional volatility, its conditional intensity and distribution of its jumps.

Chapter 5 is an example of Leveraged-Lévy model in the diffusion case. Brownian motion is the only uncertainty in this situation. Since the family of Squared Bessel processes (BESQ) is the only family of continuous semi-stable Markov processes, we study our model by incorporating BESQ processes as the Lamperti processes. The knowledge of BESQ processes enables us to derive the transition densities of the stock price process. The Maximum Likelihood Estimation as estimation of parameters in the model is carried out on time series data of $S \& P 500$ Index. In this chapter, we also study how to price options assuming that the underlying stock price follows this Leverage-Lévy process. We derive the forward Partial IntegroDifferential Equations (PIDE) and solve the problem numerically by implementing 
a finite difference algorithm which allows to price European options. Forward PIDE is preferred to backward PIDE since it obtains option prices on the whole surface of all strikes and maturities after one run of solving. By numerically solving the forward PIDE, the model parameters are calibrated to actual market data of option prices.

In Chapter 6, we consider only the pure jump Lévy processes, that is the ones without exposure to Brownian motions. It is argued in [11] that the use of a jump process with infinite activity, i.e. one allowing infinitely many jumps in any time interval, effectively subsumes the need for an additional diffusion component. We therefore replace the local diffusive risk neutral dynamics in local volatility models (Dupire 1994, [25]) (Derman and Kani 1994, [23]) by a local exposure to a Lévy process. Essentially our idea is to replace Brownian motion with a Lévy process running at what we call the local speed function. This local speed function is still a deterministic function of the level of the stock price and time. The Lévy process involved in this procedure is fixed through time, with only its speed that is space time dependent. In analogy with the local volatility function, we introduce a local speed function $A(S, t)$ that measures the speed at which the Lévy process is running at time $t$ when the stock price is at the level $S$. In the case of Brownian motion, scaling and time changing are equivalent operations by the scaling property of Brownian motion, but for general Lévy processes these are different operations. Time changing leads to tractable results while scaling is much more complicated. We study the case where the underlying Lévy process is taken to be CGMY process, which is introduced by Carr, Geman, Madan and Yor (2002, [11]). 
We implement the Generalized Methods of Moments (GMM) estimation in order to estimate the parameters in the model. Generalized Methods of Moment (GMM) estimation is one of two developments in econometrics in the 80 s that revolutionized empirical work in macroeconomics. (The other being the understanding of unit roots and cointegration). The path breaking articles on GMM were those of Hansen (1982, [33]) and Hansen and Singleton (1982, [35]). The GMM procedure chooses parameters so as to match the moments of the model to those of the data as closely as possible. Consider a set of competing models $(\mu(\cdot ; \theta), \sigma(\cdot ; \theta), \nu(d z, \cdot ; \theta))_{\theta \in \Theta}$ indexed by a finite dimensional compact set $\Theta$. Our goal is to infer which model $\theta \in \Theta$ most likely generated the observed data. For all but the most trivial examples the transition functions of the Markov process are unknown and thus classic Maximum Likelihood Estimation is infeasible. Consequently we instead consider a method of moments approach that requires knowledge only of the drift, volatility and jump characteristics of the process in terms of which the model is given. This leads us to study the relationship between the conditional expectation operator and the infinitesimal generator of time homogeneous Markov Processes.

In this thesis we also explore the precise link between option prices in Leveraged Lévy models and the related Partial Integro-Differential Equations in the case European options in exponential Lévy models. The Markov property of the price allows us to express option prices as solutions of Partial Integro-Differential Equations (PIDEs) which involve, in addition to a (possibly degenerate) second-order differential operator, a non-local integral term which requires specific treatment both at 
the theoretical and numerical level. Such partial integro-differential equations have been used by several authors to price options in model with jumps ([1], [15], [24], [50]). For example, in the paper by Hirsa and Madan (2003, [37]), PIDE for pricing American options was derived and put to numerical experiments when the log price dynamics of the underlying asset is given by the variance gamma (VG) law.

Before ending this introduction, I would like to briefly review some basic economic concepts that matter to this thesis. First is the concept of "Arbitrage". Arbitrage is any trading strategy requiring no cash input that has some probability of making profits, without any risk of a loss ([42], pg. 33). Another more formal definition: "an arbitrage opportunity is a consumption plan that is nonnegative always and strictly positive in at least one event, and has a non-positive initial cost" ([28], pg. 226). The definitions are equivalent. However, every investor wishes to earn money without risk. Whenever arbitrage opportunities are available, no investor will be satisfied with any unbounded portfolio, and no equilibrium can be obtained. Hence if this opportunity exists for some time, the investors actions (the arbitrageurs) entering in this under-priced portfolio will press the prices levels, so that prices will be adjusted until arbitrage is no longer possible. On the other hand, if there are no-arbitrage opportunities in the set of returns, then these returns can be supported in some equilibrium. Prices that do not allow arbitrage opportunities are in equilibrium.

Another important concept is "risk-neutral" pricing. We show its essence as only in the discrete state space. Every investor believes, by definition, that the subjective expected rate of return on each asset is the appropriate discount rate. 
Every investor must also agree that assets can be priced in a "risk-neutral" manner if the subjective probabilities are suitably adjusted. Furthermore, they all must agree on these adjusted probabilities, or, to the extent that the probabilities are not unique, they must agree on the possible sets. We know from Theorem 2 and 3 on pg. $55-57([43])$ that a positive pricing vector exists whenever no arbitrage condition is met, and that all investors regardless of beliefs agree on these prices or possible sets of prices. Choose a particular pricing vector $\mathbf{p}$ and define $\pi=\mathbf{p} R$, and $R=\left(\mathbf{1}^{\prime} \mathbf{p}\right)^{-1}$. We denote the vector of values of assets $\mathbf{v}$, and the state space tableau of payoffs $\mathbf{Y}$, e.g. $Y_{s, i}$ is the payoff in state $s$ on asset $i$. Since $\mathbf{v}=\mathbf{Y}^{\prime} \mathbf{p}$, we have $\mathbf{v}=\frac{\mathbf{Y}^{\prime} \pi}{R}$, or $v_{i}=\frac{\hat{E}\left(\tilde{Y}_{i}\right)}{R} . \hat{E}$ is the expectations operator with respect to the "probabilities" $\pi$. The elements of $\pi$ are called the risk-neutral probabilities, or martingale probabilities. $R$ is the corresponding risk-neutralized risk-free rate. Note that $\pi>\mathbf{0}$ ( since $\mathbf{p}>\mathbf{0}$ ), and $\mathbf{1}^{\prime} \pi=1$, we also have $\hat{E}(\tilde{\mathbf{z}})=\mathbf{Z}^{\prime} \pi=R \mathbf{1}$, where $\mathbf{Z}$ is the state space tableau of returns and by definition $\mathbf{Z}^{\prime} \mathbf{p}=\mathbf{1}$. This result is saying that, under the risk-neutral probabilities the expected rate of return on each asset is the (risk-neutralized) risk-free rate. If a risk-less asset exists with risk-free rate $r$, then $R$ must equal $r$ for any choice of risk-neutral probabilities since all pricing vectors must price the risk-less asset correctly, that is $\mathbf{p}^{\prime}(r \mathbf{1})=1$.

The Fundamental Theorem of Asset Pricing states the equivalence of the following statements:

1. Existence of a positive supporting price vector $q$.

2. The absence of arbitrage opportunities. 
3. Existence of an optimal demand for some agent who prefers more to less (i.e. an individual who has strictly positive marginal utility $u^{\prime}(\cdot)>0$.

4. Existence of positive risk-neutral probabilities and an associated risk-less rate (the Martingale property).

5. Existence of a positive state price density. 


\section{Chapter 2}

\section{LÉVY PROCESSES IN FINANCE}

Lévy processes have long been used in mathematical finance. Lévy processes can be thought of as random walks in continuous time, that is they are stochastic processes with independent and stationary increments. The best known and most important examples are the Poisson process, Brownian motion, the Cauchy process, and more generally stable processes.

Lévy processes concern many aspects of probability theory and its applications. In particular, they are prototypes of Markov Processes (actually, they form the class of space-time homogeneous Markov processes) and of semimartingales; they are also used as models in the study of queues, insurance risks, dams, and more recently in mathematical finance. From the viewpoint of functional analysis, they appear in connection with potential theory of convolution semigroups. Several books contain sections or chapters on Lévy processes, e.g. Lévy (1954, [47]), Itô (1961, [38]), Gihman and Skorohod (1975, [30]), Jacod and Shiryaev (1987, [41]), Sato (1990, [60]) (1995, [61]), Skorohod (1991, [64]) Rogers and Williams (1994, [58]),... See also surveys by Taylor (1973, [66]), Fristedt (1974, [27]) and Bingham (1975, [5]).

In this Chapter we review aspects of Lévy processes that apply to modeling financial securities and are used in later Chapters. We first introduce the well known Black-Scholes Model; then in Section 2.2 Lévy processes are studied. We introduce 
the CGMY process as an example of pure jump Lévy process. We conclude with a review of semigroups and generators for Lévy processes which are of important use in this thesis.

\subsection{The Black-Scholes Model}

\subsubsection{Stochastic Integrals and SDEs}

Stochastic integration was introduced by Itô in 1941, hence the name Itô calculus. It gives meaning to

$$
\int_{0}^{t} X_{u} d Y_{u}
$$

for suitable stochastic processes $X_{t}=(X(u), u \geq 0)$ and $Y_{t}=(Y(u), u \geq 0)$, the integrand and the integrator. Because we will take as integrators processes of infinite (unbounded) variation on every interval (e.g. Brownian motion), the first thing to note is that stochastic integrals can be quite different from classical deterministic integrals. The SDEs we encounter always have a unique solution and are of the following form:

$$
d X_{t}=a\left(X_{t}, t\right) d t+b\left(X_{t}, t\right) d Y_{t}, \quad X_{0}=x_{0}
$$

The solution to such an SDE is a stochastic process $X_{t}=(X(t), t \geq 0)$, which satisfies

$$
X(t)=\int_{0}^{t} a(X(u), u) d u+\int_{0}^{t} b(X(u), u) d Y(u), \quad X(0)=x_{0}
$$

We refer to Jacod (1979, [40]), Protter (1990, [57]) and Jacod and Shiryaev (1987, [41]) for detailed expositions on stochastic calculus. 


\subsubsection{Geometric Brownian Motion}

In the Black-Scholes model, the time evolution of a stock price $S_{t}=\left(S_{t}, t \geq 0\right)$ is modeled as follows. Consider how $S$ will change in some small time interval from the present time $t$ to a time $t+\Delta t$ in the near future. Writing $\Delta S_{t}$ for the change $S_{t+\Delta t}-S_{t}$, the return in this interval is $\Delta S_{t} / S_{t}$. It is economically reasonable to expect this return to decompose into two components, a systematic part and a random part.

Let us first look at the systematic part of the expected return. We assume that the expected return of stock over a period is proportional to the length of the period considered. This means that in a short interval of time $\left[S_{t}, S_{t+\Delta t}\right]$ of length $\Delta t$, the expected increase in $S$ is given by $\mu S_{t} \Delta t$, where $\mu$ is some parameter representing the mean rate of the return of the stock. In other words, the deterministic part of the stock return is modeled by $\mu \Delta t$.

To consider the random part of the expected return we look at the stochastic fluctuations in stock price. A reasonable assumption is that the variance of the return over the interval of time $\left[S_{t}, S_{t+\Delta t}\right]$ is proportional to the length of the interval. So, the random part of the return can be modeled by $\sigma \Delta W_{t}$, where $\Delta W_{t}$ represents the (Normally distributed) noise term (with variance $\Delta t$ ) driving the stock-price dynamics, and $\sigma>0$ is the parameter that describes how much effect the noise has, i.e. how much the stock price fluctuates. In total, the variance of the return equals $\sigma^{2} \Delta t$. Thus $\sigma$ governs how volatile the price is, and is called the volatility of the stock. 
Putting them together, we have

$$
\Delta S_{t}=S_{t}\left(\mu \Delta t+\sigma \Delta W_{t}\right), \quad S_{0}>0
$$

In the limit, as $\Delta t \rightarrow 0$, we obtain the stochastic differential equation:

$$
d S_{t}=S_{t}\left(\mu d t+\sigma d W_{t}\right), \quad S_{0}>0
$$

The above stochastic differential equation has the unique solution

$$
S_{t}=S_{0} \exp \left(\left(\mu-\frac{1}{2} \sigma^{2}\right) t+\sigma W_{t}\right)
$$

which is the functional of Brownian motion called geometric Brownian motion. Note that

$$
\ln S_{t}-\ln S_{0}=\left(\mu-\frac{1}{2} \sigma^{2}\right) t+\sigma W_{t}
$$

has a Normal $\left(t\left(\mu-\frac{1}{2} \sigma^{2}\right), \sigma^{2} t\right)$ distribution. Thus $S_{t}$ itself has a log-normal distribution. This geometric Brownian motion model and the log-normal distribution which it entails form the basis for the Black-Scholes model for stock-price dynamics in continuous time.

In Figure 2.1, the realization of a sample path of the geometric Brownian motion in $T=1$ year and with $S_{0}=100, \mu=0.05, \sigma=0.4$ is shown.

\subsubsection{The Black-Scholes Option Pricing Model}

In the early 1970s, Fisher Black, Myron Scholes ([9]) and Robert Merton ([51]) made a major breakthrough in the pricing of stock options by developing what has 
Figure 2.1: One Sample Path of Geometric Brownian Motion

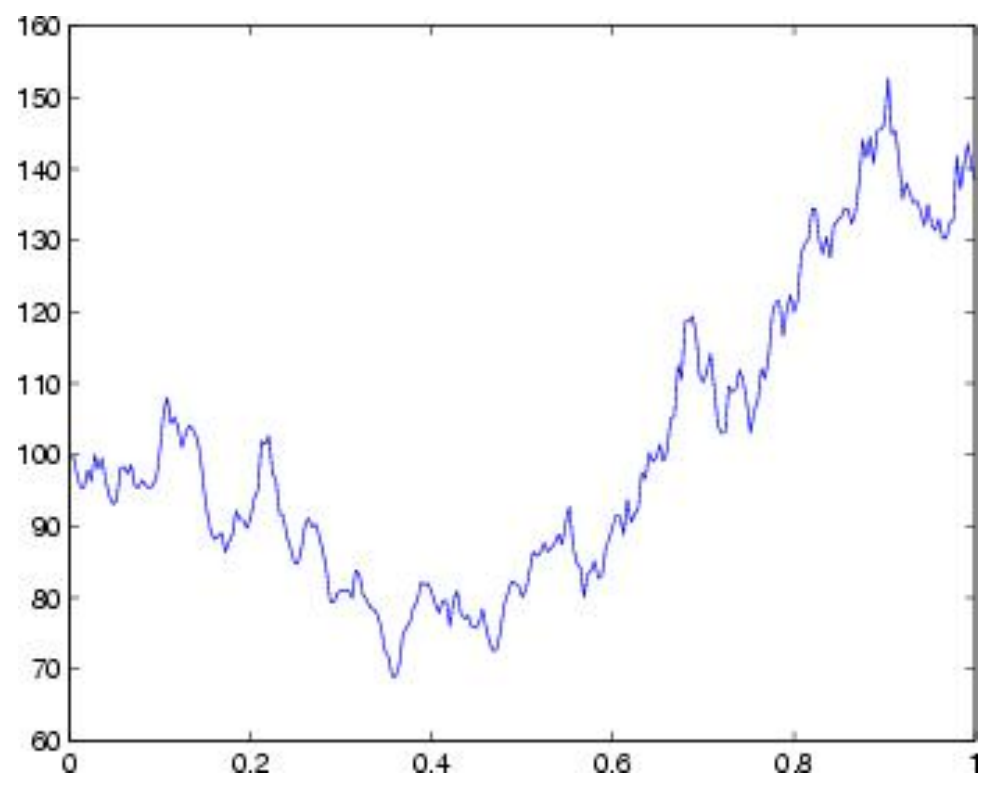

become known as the Black-Scholes model. The model has had huge influence on the way that traders price and hedge options. In 1997, the importance of the model was recognized when Myron Scholes and Robert Merton were awarded the Nobel Prize for economics.

We show how the Black-Scholes model for valuing European call and put options on a stock works.

Investors are allowed to trade continuously up to some fixed finite planning horizon $T$. The uncertainty is modeled by a filtered probability space $(\Omega, \mathcal{F}, P)$. We assume a frictionless market with two assets.

The first asset is one without risk (the bank account). Its price process is given by $B_{t}=(B(t)=\exp (r t), 0 \leq t \leq T)$. The second asset is a risky asset, usually referred to as a stock, which pays a continuous dividend yield $q \geq 0$. The price process of this stock, $S_{t}=(S(t), 0 \leq t \leq T)$, is modeled by the geometric Brownian 
motion,

$$
B(t)=\exp (r t), \quad S(t)=S(0) \exp \left(\left(\mu-\frac{1}{2} \sigma^{2}\right) t+\sigma W(t)\right)
$$

where $W_{t}=(W(t), t \geq 0)$ is a standard Brownian motion.

Note that under $P, W(t)$ has a $\operatorname{Normal}(0, t)$ and that $S_{t}=(S(t), t \geq 0)$ satisfies the SDE (2.2). The parameter $\mu$ reflects the drift and $\sigma$ models the volatility; $\mu$ and $\sigma$ are assumed to be constant over time.

We assume, as underlying filtration, the natural filtration $\mathbb{F}=\left(\mathcal{F}_{t}\right)$ generated by $W$. Consequently, the stock-price process $S_{t}=(S(t), 0 \leq t \leq T)$ follows a strictly positive adapted process. We call this market model the Black-Scholes model. It was shown by Itô (1951) that Brownian motion possesses the Predictable Representation Property. The economic relevance of the representation theorem is that it shows that the Black-Sholes model is complete, that is, that every contingent claim can be replicated by a dynamic trading strategy.

Since the Black-Scholes market model is complete, there exists only one equivalent martingale measure $Q$. It is not hard to see that under $Q$, the stock price is following a geometric Brownian motion again (Girsanov theorem). This risk-neutral stock-price process has the same volatility parameter $\sigma$, but the drift parameter $\mu$ is changed to the continuously compounded risk-free rate $r$ minus the dividend yield $q$ :

$$
S_{t}=S_{0} \exp \left(\left(r-q-\frac{1}{2} \sigma^{2}\right) t+\sigma W_{t}\right)
$$

Equivalently, we can say that under $Q$ our stock-price process $S_{t}=(S(t), 0 \leq t \leq T)$ 
satisfies the following SDE:

$$
d S_{t}=S_{t}\left((r-q) d t+\sigma d W_{t}\right), \quad S_{0}>0 .
$$

This SDE tells us that in a risk-neutral world the total return from the stock must be $r$; the dividends provide a return of $q$, the expected growth rate of the stock price, therefore, must be $r-q$.

By the risk-neutral valuation principle, the price $V_{t}$ at time $t$ of a contingent claim with payoff function $G(S(u))$ is given by

$$
V_{t}=\exp (-(T-t) r) E_{Q}\left[G(S(u)) \mid \mathcal{F}_{t}\right], \quad t \in[0, T] .
$$

Furthermore, if the payoff function depends only on the time $\mathrm{T}$ value of the stock, i.e. $G((S(u), 0 \leq u \leq T))=G(S(T))$, then formula (2.5) can be written as (for simplicity, set $t=0)$

$$
\begin{aligned}
V_{0} & =\exp (-T r) E_{Q}[G(S(T))] \\
& =\exp (-T r) E_{Q}\left[G\left(S_{0} \exp \left(\left(r-q-\frac{1}{2} \sigma^{2}\right) T+\sigma W_{T}\right)\right)\right] \\
& =\exp (-T r) \int_{-\infty}^{+\infty} G\left(S_{0} \exp \left(\left(r-q-\frac{1}{2} \sigma^{2}\right) T+\sigma x\right)\right) f_{\text {normal }}(x ; 0, T) d x .
\end{aligned}
$$

Moreover, if $G(S(T))$ is a sufficiently integrable function, then the price is also given by $V_{t}=F\left(t, S_{t}\right)$, where $F$ solves the Black-Scholes partial differential equation,

$$
\frac{\partial}{\partial t} F(t, s)+(r-q) s \frac{\partial}{\partial s} F(t, s)+\frac{1}{2} \sigma^{2} s^{2} \frac{\partial^{2}}{\partial s^{2}} F(t . s)-r F(t, s)=0, \quad F(T, s)=G(s) .
$$


This follows from the Feynman-Kac representation for Brownian motion (see for example, Bingham and Kiesel 1998, [7]).

Solving the Black-Scholes partial differential equation (2.6) is not always easy. However, in some cases it is possible to evaluate explicitly the above expected value in the risk-neutral pricing formula (2.5).

Take, for example, a European call on the stock with strike $K$ and maturity $T$, so that $G(S(T))=\left(S_{T}-K\right)^{+}$, the Black-Scholes formulas for the price $C(K, T)$ at time zero of this European call option on the stock (with dividend yield $q$ ) is given by

$$
C(K, T)=C=\exp (-q t) S_{0} N\left(d_{1}\right)-K \exp (-r T) N\left(d_{2}\right)
$$

where

$$
\begin{gathered}
d_{1}=\frac{\ln \left(S_{0} / K\right)+\left(r-q+\frac{1}{2} \sigma^{2}\right) T}{\sigma \sqrt{T}}, \\
d_{2}=\frac{\ln \left(S_{0} / K\right)+\left(r-q-\frac{1}{2} \sigma^{2}\right) T}{\sigma \sqrt{T}}=d_{1}-\sigma \sqrt{T},
\end{gathered}
$$

and $N(x)$ is the cumulative distribution function for a variable that is standard Normally distributed.

From (2.7) via put-call parity we can easily obtain the price $P(K, T)$ of the European put option on the same stock with same strike $K$ and same maturity $T$ :

$$
P(K, T)=P=-\exp (-q t) S_{0} N\left(-d_{1}\right)+K \exp (-r T) N\left(-d_{2}\right)
$$


For the call, the probability (under $Q$ ) of finishing in the money corresponds to $N\left(d_{2}\right)$. Similarly, the delta ${ }^{1}$ of the option corresponds to $N\left(d_{1}\right)$.

The Black-Scholes model has turned out to be very popular. One should bear in mind, however, that this elegant theory hinges on several crucial assumptions. We assumed that there was no market friction, such as taxes and transaction costs, and that there were no constraints on the stock holding, etc.

Moreover, empirical evidence suggests that the classical Black-Scholes model does not describe the statistical properties of financial time series very well. The two main problems are:

1. The log returns do not behave according to a Normal distribution. Instead, the analysis of univariate time series data on financial market asset returns has confirmed their long-tailedness relative to the normal distribution. Analysis of such returns using data on option prices confirms this phenomenon on a risk neutral basis, with the addition now of a marked negative skewness as well.

2. It has been observed that the volatilities or the estimated parameters of uncertainty change stochastically over time and are clustered. Many researchers have documented a negative relationship between market volatilities and the level of asset prices.

\footnotetext{
${ }^{1}$ That is, the change in the value of the option compared with the change in the value of the underlying asset.
} 


\subsection{Lévy Processes}

\subsubsection{Introduction}

One of the main problems with the Black-Scholes model is that the data suggest that the log returns of stocks are not Normally distributed. This has led to an increasing adoption of alternative processes for describing these returns. Looking at the definition of Brownian motion, we would like to have a similar process (i.e. a process with independent and stationary increments), based on a more general distribution than the Normal. However, in order to define such a stochastic process with independent and stationary increments, the distribution has to be infinitely divisible. Such processes are called Lévy processes, in honor of Paul Lévy, the pioneer of the theory.

One of the first to propose an exponential non-normal Lévy process was Mandelbrot $(1963,[49])$. He observed that the logarithm of relative price changes on financial and commodities markets exhibit a long-tailed distribution. His conclusion was that Brownian motion in $\exp \left(B_{t}\right)$ should be replaced by symmetric $\alpha$-stable Lévy motion with index $\alpha<2$. This yields a pure jump stock price process. Roughly speaking, one may envisage this process as changing its values only by jumps. Normal distributions are $\alpha$-stable distributions with $\alpha=2$, so Mandelbrot's model may be seen as a complement of the Osborne (1959, [54]) and Samuelson (1965, [59]) models. A few years after Mandelbrot's proposal, an exponential Lévy process model with a non-stable distribution was proposed by Press (1967, [56]). His log price process is a superposition of a Brownian motion and an independent com- 
pound Poisson process with normally distributed jumps. Again, the motivation was to find a model that better fit the empirically observed distribution of changes in the logarithm of stock prices. Examples of more complicated distributions, which take into account skewness and excess kurtosis, are the Variance Gamma (VG), the Normal Inverse Gaussian (NIG), the CGMY, the (Generalized) Hyperbolic Model and the Meixner distributions.

A review of financial modeling with jump processes may be found in [17]. Now let us begin to introduce Lévy processes.

\subsubsection{Definitions and Theorems}

Suppose $\phi(u)$ is the characteristic function of a distribution. If, for every positive integer $n, \phi(u)$ is also the $n$th power of a characteristic function, we say that the distribution is infinitely divisible.

Let $X_{t}=(X(t), t \geq 0)$ be a stochastic process defined on a probability space $(\Omega, \mathcal{F}, \mathcal{P})$. We say that it has independent increments if for each $n \in \mathbb{N}$ and each $0 \leq t_{1}<t_{2} \leq \cdots<t_{n+1}<\infty$ the random variables $\left(X\left(t_{j+1}\right)-X\left(t_{j}\right), 1 \leq j \leq n\right)$ are independent and that it has stationary increments if each $X\left(t_{j+1}\right)-X\left(t_{j}\right) \stackrel{d}{=} X\left(t_{j+1}-\right.$ $\left.t_{j}\right)-X(0)$

We can define for every such infinitely divisible distribution a stochastic process, $X_{t}=(X(t), t \geq 0)$, called a Lévy process, which satisfies:

(L1) $X(0)=0$ (a.s);

(L2) $X$ has independent and stationary increments; 
(L3) $X$ is stochastically continuous, i.e. for all $a>0$ and for all $s \geq 0$

$$
\lim _{t \rightarrow s} P(|X(t)-X(s)|>a)=0,
$$

such that the distribution of an increment over $[s, s+t], s, t \geq 0$, i.e. $X(t+s)-X(s)$ has $(\phi(u))^{t}$ as its characteristic function. Note that in the presence of $(L 1)$ and $(L 2)$, (L3) is equivalent to the condition

$$
\lim _{t \downarrow 0} P(|X(t)|>a)=0
$$

for all $a>0$.

We are now going to explore the relationship between Lévy processes and infinite divisibility.

Proposition 2.2.1. If $X$ is a Lévy process, then $X(t)$ is infinitely divisible for each $t \geq 0$

Proof. For each $n \in \mathbb{N}$, we can write

$$
X(t)=Y_{1}^{(n)}(t)+\cdots+Y_{n}^{(n)}(t),
$$

where each

$$
Y_{k}^{(n)}(t)=X\left(\frac{k t}{n}\right)-X\left(\frac{(k-1) t}{n}\right) .
$$

The $Y_{k}^{(n)}(t)$ are i.i.d. by $(L 2)$.

By Proposition 2.2.1, we can write $\phi_{X(t)}(u)=e^{\eta(t, u)}$ for each $t \geq 0, u \in \mathbb{R}^{d}$, where each $\eta(t, \cdot)$ is a Lévy symbol. We will see below that $\eta(t, u)=t \eta(1, u)$ for each $t \geq 0, u \in \mathbb{R}^{d}$, but first we need the following lemma. 
Lemma 2.2.2. If $X_{t}=(X(t), t \geq 0)$ is stochastically continuous, then the map $t \rightarrow \phi_{X(t)}(u)$ is continuous for each $u \in \mathbb{R}^{d}$.

Proof. For each $s, t \geq 0$ with $t \neq s$, write $X(s, t)=X(t)-X(s)$. Fix $u \in \mathbb{R}^{d}$. Since the map $y \rightarrow e^{i(u, y)}$ is continuous at the origin, given any $\epsilon>0$ we can find $\delta_{1}>0$ such that

$$
\sup _{0 \leq|y|<\delta_{1}}\left|e^{i(u, y)}-1\right|<\frac{\epsilon}{2}
$$

By stochastic continuity, we can find $\delta_{2}>0$ such that whenever $0<|t-s|<\delta_{2}$, $P\left(|X(s, t)|>\delta_{1}\right)<\frac{\epsilon}{4}$. Hence, for all $0<|t-s|<\delta_{2}$ we have

$$
\begin{aligned}
& \left|\phi_{X(t)}(u)-\phi_{X(s)}(u)\right| \\
= & \left|\int_{\Omega} e^{i(u, X(s)(\omega))}\left[e^{i(u, X(s, t)(\omega))}-1\right] P(d \omega)\right| \\
\leq & \int_{\mathbb{R}^{d}}\left|e^{i(u, y)}-1\right| p_{X(s, t)}(d y) \\
= & \int_{B_{\delta_{1}(0)}}\left|e^{i(u, y)}-1\right| p_{X(s, t)}(d y)+\int_{B_{\delta_{1}(0) c}}\left|e^{i(u, y)}-1\right| p_{X(s, t)}(d y) \\
\leq & \sup _{0 \leq|y|<\delta_{1}}\left|e^{i(u, y)}-1\right|+2 P\left(|X(s, t)|>\delta_{1}\right) \\
< & \epsilon .
\end{aligned}
$$

The result follows.

Theorem 2.2.3. If $X$ is a Lévy process, then

$$
\phi_{X(t)}(u)=e^{t \eta(u)}
$$

for each $u \in \mathbb{R}^{d}, t \geq 0$, where $\eta$ is the Lévy symbol of $X(1)$.

Proof. Suppose that $X$ is a Lévy process and that, for each $u \in \mathbb{R}^{d}, t \geq 0$. Define 
$\phi_{u}(t)=\phi_{X(t)}(u)$; then by $(L 2)$ we have for all $s \geq 0$

$$
\begin{aligned}
\phi_{u}(t+s) & =E\left(e^{i(u, X(t+s))}\right) \\
& =E\left(e^{i(u, X(t+s)-X(s))} e^{i(u, X(s))}\right) \\
& =E\left(e^{i(u, X(t+s)-X(s))}\right) E\left(e^{i(u, X(s))}\right) \\
& =\phi_{u}(t) \phi_{u}(s) .
\end{aligned}
$$

Now $\phi_{u}(0)=1$ by $(L 1)$, and from (L3) and Lemma 2.2.2 we have that the map $t \rightarrow \phi_{u}(t)$ is continuous. However, the unique continuous solution to (2.8) and initial condition $\phi_{u}(0)=1$ is given by $\phi_{u}(t)=e^{t \alpha(u)}$, where $\alpha: \mathbb{R}^{d} \rightarrow \mathbb{C}$ (see e.g. Bingham et al. [6], pg. 4-6). Now by Proposition 2.2.1 X(1) is infinitely divisible, hence $\alpha$ is a Lévy symbol and the result follows.

We now have the Lévy-Khinchine formula for a Lévy process $X_{t}=(X(t), t \geq$ $0)$,

$E\left(e^{i(u, X(t))}\right)=\exp \left(t\left\{i(b, u)-\frac{1}{2}(u, A u)+\int_{\mathbb{R}^{d}-\{0\}}\left[e^{i(u, x)}-1-i(u, x) \chi_{\hat{B}}(x)\right] \nu(d x)\right\}\right)$

for each $t \geq 0, u \in \mathbb{R}^{d}$, where $(b, A, \nu)$ are the characteristics of $X(1)$. In the one dimensional case, Lévy symbol $\eta(u)$ is

$$
\eta(u)=i \gamma u-\frac{1}{2} \sigma^{2} u^{2}+\int_{-\infty}^{+\infty}\left[e^{i u x}-1-i u x \chi_{\hat{B}}(x)\right] \nu(d x)
$$

where $\gamma \in \mathbb{R}, \sigma^{2} \geq 0$ and $\nu$ is a measure on $\mathbb{R} \backslash\{0\}$ with

$$
\int_{-\infty}^{+\infty} \inf \left\{1, x^{2}\right\} \nu(d x)=\int_{-\infty}^{+\infty}\left(1 \wedge x^{2}\right) \nu(d x)<\infty .
$$

This infinitely divisible distribution has a triplet of Lévy characteristics, or Lévy triplet in short, $\left(\gamma, \sigma^{2}, \nu(d x)\right)$, and the measure $\nu$ is called the Lévy measure of $X$. 
If the Lévy measure is of the form $\nu(d x)=u(x) d x$, we call $u(x)$ the Lévy density. The Lévy density has the same mathematical requirement as a probability density, except that it does not need to be integrable and must have zero mass at the origin.

From the Lévy-Khintchine formula, we see that in general, a Lévy process consists of three independent parts: a linear deterministic part, a Brownian part and a pure jump part. The Lévy measure $\nu(d x)$ dictates how the jumps occur. Jumps of sizes in the set $A$ occur according to a Poisson process with intensity parameter $\int_{A} \nu(d x)$.

A subordinator is a nonnegative nondecreasing Lévy process. It is not hard to see that as such a subordinator has no Brownian part $\left(\sigma^{2}=0\right)$, a nonnegative drift and a Lévy measure which is zero on the negative half-line. Note that a subordinator is nondecreasing and always of finite variation.

Let us now discuss more about the path properties of Lévy processes. If $\sigma^{2}=0$ and $\int_{-1}^{+1}|x| \nu(d x)<\infty$, it follows from standard Lévy process theory that the process is of finite variation. In that case the Lévy symbol can be re-expressed as

$$
\eta(u)=i \gamma^{\prime} u+\int_{-\infty}^{+\infty}(\exp (i u x)-1) \nu(d x)
$$

for some $\gamma^{\prime}$, which we call the drift coefficient. In the finite-variation case, we can decompose the process into the difference of two increasing processes.

If $\sigma^{2}=0$ and $\int_{-1}^{+1} \nu(d x)<\infty$, there are finitely many jumps in any finite interval. We say that the process is of finite activity.

Because the Brownian motion is of infinite variation, a Lévy process with a Brownian component is of infinite variation. A pure jump Lévy process, i.e. 
one with no Brownian component $\left(\sigma^{2}=0\right)$, is of infinite variation if and only if

$\int_{-1}^{+1}|x| \nu(d x)=\infty$. In that case special attention has to be paid to the small jumps. Basically, the sum of all jumps smaller than some $\epsilon>0$ does not converge. However, the sum of the jumps compensated by their mean does converge. This peculiarity leads to the necessity of the compensator term $i u x \chi_{\hat{B}}(x)$ in $(2.9)$.

There are many examples of Lévy processes and many of them are popular as building blocks of stock price models. A list of Lévy processes together with their density functions, their characteristic functions and their Lévy triplets and other important properties can be found in [62]. We now turn our focus to an example of pure jump Lévy processes, CGMY processes, that we use later as the underlying Lévy processes when constructing our stock price model.

\subsubsection{The CGMY Process}

In order to obtain a more flexible process than the Variance Gamma (VG) process, one allowing finite activity, infinite activity and infinite variation, the additional parameter $Y$ was introduced by Carr, Madan, Geman and Yor in 2002 $([11])$.

The $\operatorname{CGMY}(C, G, M, Y)$ distribution is a four-parameter distribution, with characteristic function

$$
\phi_{C G M Y}(u ; C, G, M, Y)=\exp \left(C \Gamma(-Y)\left((M-i u)^{Y}-M^{Y}+(G+i u)^{Y}-G^{Y}\right)\right) .
$$

The CGMY distribution is infinitely divisible and we can define the CGMY Lévy 
process

$$
X_{t}^{(C G M Y)}=\left(X(t)^{(C G M Y)}, t \geq 0\right)
$$

as the process which starts at zero with independent and stationary increments and whose increment over a time interval of length $s$ follows a $\operatorname{CGMY}(s C, G, M, Y)$ distribution; in other words, the characteristic function of $X_{t}^{(C G M Y)}$ is given by

$$
\begin{aligned}
E\left[\exp \left(i u X_{t}^{(C G M Y)}\right)\right] & =\phi_{C G M Y}(u ; t C, G, M, Y) \\
& =\left(\phi_{C G M Y}(u ; C, G, M, Y)\right)^{t} \\
& =\exp \left(C t \Gamma(-Y)\left((M-i u)^{Y}-M^{Y}+(G+i u)^{Y}-G^{Y}\right)\right) .
\end{aligned}
$$

The Lévy measure for the CGMY process is give by

$$
\nu_{C G M Y}(d x)= \begin{cases}C \exp (G x)(-x)^{-1-Y} d x, & x<0, \\ C \exp (-M x) x^{-1-Y} d x, & x>0 .\end{cases}
$$

The first parameter of the Lévy triplet equals

$$
\gamma=C\left(\int_{0}^{1} \exp (-M x) x^{-Y} d x-\int_{-1}^{0} \exp (G x)|x|^{-Y} d x\right)
$$

The range of the parameters is restricted to $C, G, M>0$ and $Y<2$. Choosing the $Y$ parameters greater than or equal to two does not yield a valid Lévy measure.

The CGMY process is a pure jump process, that is, it contains no Brownian part. The path behavior is determined by the $Y$ parameters. If $Y<0$, the paths have finite jumps in any finite interval; if not, the paths have infinitely many jumps in any finite time interval, i.e. the process has infinite activity. Moreover, if the $Y$ parameters lie in the interval $[1,2)$, the process is of infinite variation. 
The Variance Gamma distribution (process) is a special case of the CGMY distribution (process). When $Y=0$, the CGMY reduces to VG.

\subsection{Semigroups and Their Generators}

There are many good books on semigroup theory and we have followed Davies [21] very closely. Let $B$ be a real Banach space and $L(B)$ be the algebra of all bounded linear operators on $B$. A one-parameter semigroup of contractions on $B$ is a family of bounded, linear operators $\left(T_{t}, t \geq 0\right)$ on $B$ for which

(1) $T_{s+t}=T_{s} T_{t}$ for all $s, t \geq 0$,

(2) $T_{0}=I$,

(3) $\left\|T_{t}\right\| \leq 1$ for all $t \geq 0$,

(4) the map $t \rightarrow T_{t}$ from $\mathbb{R}^{+}$to $L(B)$ is strongly continuous at zero, i.e. $\lim _{t \downarrow} \| T_{t} \psi-$ $\psi \|=0$ for all $\psi \in B$

From now on we will say that $\left(T_{t}, t \geq 0\right)$ is a semigroup whenever it satisfies the above conditions.

In the context of Markov process, we associate each Markov process $X$ a family of operators $\left(T_{t}, t \geq 0\right)$ on $B$ by the prescription

$$
\left(T_{t} f\right)(x)=E(f(X(t)) \mid X(0)=x)
$$

for each $t \geq 0, f \in B, x \in \mathbb{R}$. If $X$ admits transition probabilities $p_{s, t}$ defined as

$$
p_{s, t}(x, A)=\left(T_{s, t} \chi_{A}\right)(x)=P(X(t) \in A \mid X(s)=x),
$$


we then have

$$
\left(T_{t} f\right)(x)=\int_{\mathbb{R}} f(y) p_{0, t}(x, d y)
$$

for each $t \geq 0, f \int B, x \in \mathbb{R}$.

Now let $\left(T_{t}, t \geq 0\right)$ be an arbitrary semigroup in a Banach space $B$. We define

$$
D_{A}=\left\{\psi \in B ; \exists \phi_{\psi} \in B \text { such that } \lim _{t \downarrow 0}\left\|\frac{T_{t} \psi-\psi}{t}-\phi_{\psi}\right\|=0\right\} .
$$

It is easy to verify that $D_{A}$ is a linear space and thus we may define a linear operator $A$ in $B$, with domain $D_{A}$, by the prescription

$$
A \psi=\phi_{\psi}
$$

so that, for each $\psi \in D_{A}$,

$$
A \psi=\lim _{t \downarrow 0} \frac{T_{t} \psi-\psi}{t}
$$

We call $A$ the infinitesimal generator, or sometimes just the generator, of the semigroup $\left(T_{t}, t \geq 0\right)$. In the case where $\left(T_{t}, t \geq 0\right)$ is the Feller semigroup associated with a Feller process $X_{t}=(X(t), t \geq 0)$, we sometimes call $A$ the generator of $X$. Before we focus on the semigroups and their generators for Lévy processes, we review some important technical results about semigroups and their generators in the general framework.

Lemma 2.3.1. $\psi(t) \in D_{A}$ for each $t \geq 0, \psi \in B$ and

$$
A \psi(t)=T_{t} \psi-\psi
$$


Proof. Apply the continuity of $T_{s}$ together with the semigroup condition (1) to write

$$
T_{s} \psi(t)=\int_{0}^{t} T_{s+u} \psi d u
$$

Using this and the fundamental theorem of calculus, the fact that $T_{0}=I$ and a standard change of variable, we find for each $t \geq 0$,

$$
\begin{aligned}
\lim _{h \downarrow 0} \frac{1}{h}\left[T_{h} \psi(t)-\psi(t)\right] & =\lim _{h \downarrow 0}\left(\frac{1}{h} \int_{0}^{t} T_{h+u} \psi d u-\frac{1}{h} \int_{0}^{t} T_{u} \psi d u\right) \\
& =\lim _{h \downarrow 0}\left(\frac{1}{h} \int_{h}^{t+h} T_{u} \psi d u-\frac{1}{h} \int_{0}^{t} T_{u} \psi d u\right) \\
& =\lim _{h \downarrow 0}\left(\frac{1}{h} \int_{t}^{t+h} T_{u} \psi d u-\frac{1}{h} \int_{0}^{h} T_{u} \psi d u\right) \\
& =T_{t} \psi-\psi,
\end{aligned}
$$

and the required result follows.

\section{Theorem 2.3.2.}

(1) $D_{A}$ is dense in $B$.

(2) $T_{t} D_{A} \subseteq D_{A}$ for each $t \geq 0$.

(3) $T_{t} A \psi=A T_{t} \psi$ for each $t \geq 0, \psi \in D_{A}$.

Proof. (1) By Lemma 2.3.1, $\psi(t) \in D_{A}$ for each $t \geq 0, \psi \in B$, but by the fundamental theorem of calculus, $\lim _{t \downarrow 0}(\psi(t) / t)=\psi$; hence $D_{A}$ is dense in $B$ as required. For (2) and (3), suppose that $\psi \in D_{A}$ and $t \geq 0$; then, by the definition of $A$ and the continuity of $T_{t}$, we have

$$
\begin{aligned}
A T_{t} \psi & =\left[\lim _{h \downarrow 0} \frac{1}{h}\left(T_{h}-I\right)\right] T_{t} \psi=\lim _{h \downarrow 0} \frac{1}{h}\left(T_{t+h}-T_{t}\right) \psi \\
& =T_{t}\left[\lim _{h \downarrow 0} \frac{1}{h}\left(T_{h}-I\right)\right] \psi=T_{t} A \psi .
\end{aligned}
$$


The strong derivative in $B$ of the mapping $t \rightarrow T_{t} \psi$, where $\psi \in D_{A}$, is given by

$$
\frac{d}{d t} T_{t} \psi=\lim _{h \downarrow 0} \frac{T_{t+h} \psi-T_{t} \psi}{h}
$$

From the proof of Theorem 2.3.2, we deduce that

$$
\frac{d}{d t} T_{t} \psi=A T_{t} \psi
$$

More generally, it can be shown that $t \rightarrow T_{t} \psi$ is the unique solution of the following initial-value problem in Banach space:

$$
\frac{d}{d t} u(t)=A u(t), \quad u(0)=\psi
$$

see e.g. Davies [21], pg. 5. This justifies the notation $T_{t}=e^{t A}$.

We now can investigate the application of the analytical concepts of semigroups and their generators to Lévy processes. To begin, we introduce a Lévy process $X_{t}=(X(t), t \geq 0)$ that is adapted to a given filtration $\left(\mathcal{F}_{t}, t \geq 0\right)$ in a probability space $(\Omega, \mathcal{F}, \mathcal{P})$. The mapping $\eta$ is the Lévy symbol of $X$, so that

$$
E\left(e^{i u X(t)}\right)=e^{t \eta(u)}
$$

for all $u \in \mathbb{R}$. We know that $\eta$ is a continuous, hermitian, conditionally positive mapping from $\mathbb{R}$ to $\mathbb{C}$ that satisfies $\eta(0)=0$ and whose precise form is given by the Lévy-Khintchine formula. For each $t \geq 0, q_{t}$ will denote the law of $X(t)$. Since every Lévy process is a Feller process ( see proof in e.g. [3] pg. 126-127) and if $\left(T_{t}, t \geq 0\right)$ is the associated Feller semigroup then 


$$
\left(T_{t} f\right)(x)=\int_{\mathbb{R}} f(x+y) q_{t}(d y)
$$

for each $f \in B_{b}(\mathbb{R}), x \in \mathbb{R}, t \geq 0$, i.e.

$$
\left(T_{t} f\right)(x)=E(f(X(t)+x)) .
$$

This is not hard to see since by definition $T_{t} f(x)=E(f(X(t)) \mid X(0)=x)=$ $\int f(y) p_{0, t}(x, d y)$, where $p_{s, t}(x, A)$ are transition probabilities defined as

$$
p_{s, t}(x, A)=\left(T_{s, t} \chi_{A}\right)(x)=P(X(t) \in A \mid X(s)=x),
$$

and moreover, Lévy process $X$ admits the relation

$$
p_{s, t}(x, A)=q_{t-s}(A-x),
$$

for each $0 \leq 0<t<\infty$,

We now turn our attention to the infinitesimal generators of Lévy processes. The next theorem is of great importance in the analytic study of Lévy processes and of their generalizations. Let $f$ be in the Schwartz space of rapidly decreasing functions $S\left(\mathbb{R}^{d}\right)$. We recall that $f$ has its Fourier transform $\hat{f} \in S\left(\mathbb{R}^{d}, \mathbb{C}\right)$, where

$$
\hat{f}(u)=(2 \pi)^{-d / 2} \int_{\mathbb{R}^{d}} e^{-i(u, x)} f(x) d x
$$

for all $u \in \mathbb{R}^{d}$, and the Fourier inversion formula yields

$$
f(x)=(2 \pi)^{-d / 2} \int_{\mathbb{R}^{d}} e^{i(u, x)} \hat{f}(u) d u
$$

for each $x \in \mathbb{R}^{d}$. 
Theorem 2.3.3. Let $X$ be a Lévy process with Lévy symbol $\eta$ and characteristics $(b, a, \nu)$. Let $\left(T_{t}, t \geq 0\right)$ be the associated Feller semigroup and $A$ be its infinitesimal generator.

(1) For each $t \geq 0, f \in S\left(\mathbb{R}^{d}\right), x \in \mathbb{R}^{d}$,

$$
\left(T_{t} f\right)(x)=(2 \pi)^{-d / 2} \int_{\mathbb{R}^{d}} e^{i(u, x)} e^{\operatorname{t\eta }(u)} \hat{f}(u) d u,
$$

so that $T_{t}$ is a pseudo-differential operator with symbol $e^{t \eta}$.

(2) For each $f \in S\left(\mathbb{R}^{d}\right), x \in \mathbb{R}^{d}$,

$$
(A f)(x)=(2 \pi)^{-d / 2} \int_{\mathbb{R}^{d}} e^{i(u, x)} \eta(u) \hat{f}(u) d u,
$$

so that $A$ is a pseudo-differential operator with symbol $\eta$.

(3) For each $f \in S\left(\mathbb{R}^{d}\right), x \in \mathbb{R}^{d}$,

$$
(A f)(x)=b^{i} \partial_{i} f(x)+\frac{1}{2} a^{i j} \partial_{i} \partial_{j} f(x)+\int_{\mathbb{R}^{d}-\{0\}}\left[f(x+y)-f(x)-y^{i} \partial_{i} f(x) \chi_{\hat{B}}(y)\right] \nu(d y) .
$$

Proof of this theorem can be found in [3] pg. 139-140.

As finishing this chapter, we shall emphasize that the knowledge of Lévy processes is very rich and their applications in many areas has drawn more and more attention in to the study of Lévy processes. Our study here in this chapter has only focused on the aspects of Lévy processes which matter to this thesis the most. The next chapter will be dedicated to the study of semi-stable stochastic processes and will eventually lead us to the important relation between semi-stable Markov processes and Lévy processes, established by Lamperti ([45]). 


\section{Chapter 3}

\section{SEMI-STABLE MARKOV PROCESSES}

A classical result, due to John Lamperti, establishes a one-to-one correspondence between a class of strictly positive Markov processes that are semi-stable, and the class of one-dimensional Lévy processes. This correspondence is obtained by suitably time-changing the exponential of the Lévy process. This relation also makes our attempt to "leverage" the Lévy processes become possible as in Chapter 4 we show that our stock model constructed by time change the Lévy process can in fact be expressed as some semi-stable Markov process raised to a power.

In this chapter Lamperti's 1962 and 1972 papers ([44], [45]) are studied. We first introduce the definitions and properties of the class of semi-stable processes. Then we focus on the study of Markov processes which are semi-stable. The bijection between exponentials of Lévy processes and semi-stable Markov processes will be stated as the main theorem in Section 3.2.2. We also include an introduction of Bessel squared processes since the family of Bessel squared processes is the only

family of continuous semi-stable Markov processes. Because of this special role of Bessel squared processes, they are exclusively included in the construction of our diffusion model of leveraged Lévy in Chapter 5. 


\subsection{Semi-Stable Stochastic Processes}

An interesting chapter in modern probability theory began with the search for all the possible limit distributions for sums of independent, identically-distributed random variables. The result, the theory of the stable laws, generalizes and illuminates the original examples of normal convergence with which the problem originated. In [44], John Lamperti formalized and studied an analogous situation in the theory of stochastic processes. It is natural to raise the following question: Which processes can similarly occur as limits upon subjecting a fixed stochastic process to infinite contractions of its time and space scales? It is essentially this class of processes which Lamperti terms semi-stable. The name is intended to suggest the analogy with the theory of stable laws, and in fact is rendered more appropriate by the fact that a semi-stable process, if it is assumed to have independent increments, must actually be a stable one.

\subsubsection{Definitions and Theorems}

All the processes considered in this section have states in Euclidean space of $s$ dimensions, non-negative time parameter, and are continuous, i.e.

$$
\lim _{h \rightarrow 0} P\left(\left\|x_{t+h}-x_{t}\right\|>\epsilon\right)=0
$$

for every $t \geq 0, \epsilon>0$. We shall speak of a proper process if $x_{t}$ has a non-degenerate distribution for every $t>0$.

Definition 3.1.1. Two processes $\left\{x_{t}\right\}$ and $\left\{y_{t}\right\}$ belong to the same type (denoted 
$\left.\left\{x_{t}\right\} \sim\left\{y_{t}\right\}\right)$ if there exist constants $b, c$, respectively a positive number and an $s$-vector, such that $\left\{x_{t}\right\} \approx\left\{b y_{t}+c\right\}$ (this indicates $\left\{x_{t}\right\}$ and $\left\{b y_{t}+c\right\}$ have the same state space and finite-dimensional distribution functions).

It is easily verified that this is an equivalence relation, and that the Markov property, stationary transition probabilities, and the continuity condition (3.1) hold either for all processes of a given type or for none.

Definition 3.1.2. A process $\left\{x_{t}\right\}$ is semi-stable if it obeys the condition (3.1) and if for every $a>0,\left\{x_{a t}\right\} \sim\left\{x_{t}\right\}$.

The semi-stable property can be rephrased by saying there exist functions $b(a)$ with positive real values, and $c(a)$ with values in $\mathbb{R}_{s}$, such that for every $a>0$, $\left\{x_{a t}\right\}$ and $\left\{b(a) x_{t}+c(a)\right\}$ are the same process. This property also holds for all or none of the members of a give type, so that one can speak of a semi-stable type of processes. The first theorem which we state without proof (proof can be found in [44]), illuminates these notions somewhat.

Theorem 3.1.1. If $\left\{x_{t}\right\}$ is a proper semi-stable process, then

$$
b(a)=a^{\alpha} \text { for some } \alpha \geq 0 \text {. }
$$

If $\alpha \neq 0$, the distribution of $x_{0}$ is concentrated at a point $\omega$ and

$$
c(a)=\omega\left(1-a^{\alpha}\right) .
$$

If $\alpha=0$, then $c(a) \equiv 0$ and the process is trivial in the sense that $x_{t}=x_{0}$ a.s. for each $t$. 
Remark 3.1.1. The constant $\alpha$ is easily seen to be the same for all processes of the same type; we accordingly can and will speak of a semi-stable process or type of order $\alpha$. We observe that each nontrivial semi-stable type of order $\alpha$ contains a process with $x_{0}=0$ and such that $\left\{x_{a t}\right\} \approx\left\{a^{\alpha} x_{t}\right\}$. Thus in trying to find or classify semi-stable processes these assumptions may be imposed without loss of generality.

Remark 3.1.2. It is also easy to establish a one-to-one correspondence between the processes of one order and those of another; in fact, if $\left\{x_{t}\right\}$ is semi-stable of order $\alpha>0$, and $x_{0}=0$, then the process $\left\{y_{t}\right\}$ defined by

$$
y_{t}=\left\|x_{t}\right\|^{\beta / \alpha-1} x_{t}
$$

is semi-stable of order $\beta>0$.

Proof.

$$
\begin{aligned}
\left\{y_{a t}\right\} & \approx\left\{\left\|x_{a t}\right\|^{\beta / \alpha-1} x_{a t}\right\} \\
& \approx\left\{a^{\beta-\alpha}\left\|x_{t}\right\|^{\beta / \alpha-1} a^{\alpha} x_{t}\right\} \\
& \approx\left\{a^{\beta}\left\|x_{t}\right\|^{\beta / \alpha-1} x_{t}\right\} \\
& \approx\left\{a^{\beta} y_{t}\right\}
\end{aligned}
$$

The point of all this is that the semi-stable processes defined above are the only processes which can result from certain limiting operations. Let $\left\{X_{t}\right\}$ be a discrete or continuous time, $s$-dimensional stochastic process. We next assume that there exist real and vector valued functions $0<f(\xi) \nearrow \infty, g(\xi)$ and a proper 
$s$-dimensional process $\left\{x_{t}\right\}$ satisfying assumption (3.1) such that

$$
\lim _{\xi \rightarrow \infty}\left\{\frac{X_{\xi t}+g(\xi)}{f(\xi)}\right\}=\left\{x_{t}\right\}
$$

in the sense of convergence of finite-dimensional joint distributions, i.e.,

$$
\lim _{\xi \rightarrow \infty} P\left(\frac{X_{\xi t_{1}}+g(\xi)}{f(\xi)} \leq x_{1}, \ldots, \frac{X_{\xi t_{n}}+g(\xi)}{f(\xi)} \leq x_{n}\right)=P\left(x_{t_{1}} \leq x_{1}, \ldots, x_{t_{n}} \leq x_{n}\right)
$$

The main result of [44] (see proof of this theorem in pg.71-73, [44]), is contained in Theorem 3.1.2.

Theorem 3.1.2. If (3.6) holds, then process $\left\{x_{t}\right\}$ is semi-stable, and

$$
f(\xi)=\xi^{\alpha} L(\xi), \quad g(\xi)=\omega(\xi) \xi^{\alpha} L(\xi)
$$

where $\alpha>0, L(\xi)$ is a slowly varying function ${ }^{1}$, and the vector valued function $\omega(\xi)$ has a limit $\omega$ as $\xi \rightarrow \infty$. The order of $\left\{x_{t}\right\}$ is then $\alpha$, and $x_{0}=\omega$. Conversely, every semi-stable process of positive order is such a limit for some process $\left\{X_{t}\right\}$.

Remark 3.1.3. It is obvious that every semi-stable process arises as a limit of this sort. (The process $\left\{X_{t}\right\}$ may be chosen to be $\left\{x_{t}\right\}$ itself.) In other words, the semi-stable processes form exactly the class of possible "asymptotes" which can be obtained by taking some fixed random process and expanding indefinitely the unites in which space and time are measured. This is the basic reason, why such processes play a very large role in many aspects of probability theory and its applications.

\footnotetext{
${ }^{1}$ That is, $L(\xi)$ is positive and satisfies $L(c \xi) / L(\xi) \rightarrow 1$ as $\xi \rightarrow \infty$ for any $c>0$.
} 
Remark 3.1.4. If $\left\{y_{\tau}\right\}$ is a strictly stationary process, $-\infty<\tau<\infty$, continuous in the sense (3.1), and if for some $\alpha>0$

$$
x_{t}=t^{\alpha} y_{\ln t} \quad \text { for } t>0, x_{0}=0 \text {, }
$$

then $\left\{x_{t}\right\}$ is semi-stable of order $\alpha$. Conversely, every nontrivial semi-stable process with $x_{0}=0$ is obtained in this way from some stationary process $\left\{y_{\tau}\right\}$.

The proof simply consists of noticing that if $\left\{y_{\tau}\right\}$ is stationary, then

$$
\left\{x_{a t}\right\} \approx\left\{a^{\alpha} t^{\alpha} y_{\ln t+\ln a}\right\} \approx\left\{a^{\alpha} t^{\alpha} y_{\ln t}\right\} \approx\left\{a^{\alpha} x_{t}\right\}
$$

while if $\left\{x_{a t}\right\} \approx\left\{a^{\alpha} x_{t}\right\}$, then

$$
\left\{y_{\tau+\sigma}\right\} \approx\left\{e^{-\alpha \tau} e^{-\alpha \sigma} x_{e^{\tau} e^{\sigma}}\right\} \approx\left\{e^{-\alpha \tau} x_{e^{\tau}}\right\} \approx\left\{y_{\tau}\right\}
$$

which in short proves: if $\left\{x_{t}\right\}$ is semi-stable of order $\alpha>0$, then $\left\{y_{\tau}=e^{-\alpha \tau} x_{e^{\tau}}\right\}$ is strictly stationary.

One instance of (3.9) has been used by Doob to deduce properties of the (stationary) Ornstein-Uhlenbeck velocity process from those of the Wiener process.

The most interesting class of semi-stable processes is the class of Markov processes with stationary transition probabilities. The correspondence (3.9) does preserve the Markov property but not stationarity. In the example of Doob mentioned above, for instance, only $\alpha=1 / 2$ gives and $\left\{x_{t}\right\}$ with stationary transition probabilities, although $\left\{x_{t}\right\}$ is semi-stable and Markov for all values of $\alpha>0$. Section 3.2 will discuss semi-stable Markov processes in more detail. 


\subsubsection{Examples of Semi-Stable Processes}

\section{Example 1. Independent increments.}

A remark about terminology is first called for. In [46], P. Lévy defines a stable distribution function as one such that if $X_{1}$ and $X_{2}$ are independent random variables with the given distribution, then for every $a_{1}>0, a_{2}>0$ there exists $a_{3}>0$ satisfying

$$
a_{1} X_{1}+a_{2} X_{2}=a_{3} X_{3}
$$

where $X_{3}$ again has the given distribution. Lévy calls the distribution quasi-stable if (3.10) holds with a constant (depending on $a_{1}$ and $a_{2}$ ) added to the right-hand side. This distribution is not usually maintained today, and all of these laws are simply called stable. However, we will now show that a process $\left\{x_{t}\right\}$ with stationary independent increments and with $x_{0}=0$ is semi-stable if and only if the increments have distributions which are stable in P. Lévy's sense. If the stable distribution is of index $\gamma$, the order of $\left\{x_{t}\right\}$ is $1 / \gamma$.

To prove this, notice that if the process is semi-stable of order $\alpha$ and $x_{0}=0$, then

$$
\phi_{t}(\lambda)=E\left(e^{i\left(\lambda, x_{t}\right)}\right)=E\left(e^{i t^{\alpha}\left(\lambda, x_{1}\right)}\right)=\phi_{1}\left(t^{\alpha} \lambda\right)
$$

Since it also has stationary independent increments, we find that

$$
\phi_{1}\left(t^{\alpha} \lambda\right) \phi_{1}\left(s^{\alpha} \lambda\right)=\phi_{t}(\lambda) \phi_{s}(\lambda)=\phi_{t+s}(\lambda)=\phi_{1}\left[(t+s)^{\alpha} \lambda\right]
$$

This, if $\alpha \neq 0$, implies that the distribution of $x_{1}$ (and so of all increments) satisfies 
(3.10) and so is stable in Lévy's sense. The case $\alpha=0$ is trivial, for all increments are then 0 by Theorem 3.1.1.

In one dimension the general form of a characteristic function which is stable of index $\gamma$ in the sense of (3.10) is as follows:

$$
\ln \phi(t)=\left\{\begin{array}{cc}
-B|\lambda|^{\gamma}\left(1+\operatorname{sgn}(\lambda) i C \tan \frac{\pi \gamma}{2}\right), & 0<\gamma<2, \gamma \neq 1, \\
-B|\lambda|+i A \lambda, & \gamma=1, \\
-B \lambda^{2}, & \gamma=2 .
\end{array}\right.
$$

It is easily checked that each of these laws lead to an additive process which is semi-stable of order $1 / \gamma$, proving the converse part of the above statement when $s=1$. The same approach of direct verification, coupled with knowledge of the form of a stable law in several dimensions [46], suffices also for the multidimensional cases.

Remark 3.1.5. s-dimensional Brownian motion is semi-stable of order $1 / 2$, and more generally any direct product of semi-stable processes of order $\alpha$ is itself semi-stable of order $\alpha$.

Example 2. Zeros of a semi-stable process.

Suppose that $\left\{x_{t}\right\}$ is a measurable strong Markov process with $x_{0}=0$, and define

$$
y_{t}=t-\sup \left\{\tau \mid \tau \leq t, x_{\tau}=0\right\}
$$

Then $\left\{y_{t}\right\}$ is a Markov process whose paths consist of a collection of straight-line segments of slope 1 with left ends on the $t$-axis, plus the remaining points of the 
axis itself. It is very easy to see that if $\left\{x_{t}\right\}$ is semi-stable of any order, $\left\{y_{t}\right\}$ is semi-stable of order one.

\subsection{Semi-Stable Markov Processes}

As introduced in 3.1, a real valued stochastic process $\left\{x_{t}\right\}$, continuous in probability and with $x_{0}=0$, is called semi-stable if there is a constant $\alpha>0$ (called the order of the process) such that for every $\alpha>0$ the random functions $\left\{x_{a t}\right\}$ and $\left\{a^{\alpha} x_{t}\right\}$ have the same joint distributions. If $\left\{x_{t}\right\}$ is Markovian with the stationary transition function $P_{t}(x, E)$, it is obvious that this condition holds provided that $x_{0}=0$ and that

$$
P_{a t}(x, E)=P_{t}\left(a^{-\alpha} x, a^{-\alpha} E\right)
$$

for all $a>0, t>0, x \in \mathbb{R}$, and all measurable sets $E$. Markov processes whose transition probabilities satisfy (3.12), and which fulfill certain regularity conditions stated in [45] section 2, are the object of study in the rest of this chapter.

\subsubsection{General Preliminaries}

Definition 3.2.1. A function $P_{t}(x, E)$ is a semi-stable transition function provided that (i) it is a conservative Markov transition function in the sense of ([26], pg. 47) with respect to the state space $\left(\mathbb{R}^{+}, \mathcal{B}^{+}\right)$consisting of $[0, \infty]$ and its Borel subsets; that (ii) the semi-stable condition

$$
P_{a t}(x, E)=P_{t}\left(a^{-\alpha} x, a^{-\alpha} E\right)
$$


holds for some $\alpha>0$, all $a>0, t>0, x \in \mathbb{R}^{+}, E \in \mathcal{B}^{+}$; and that (iii) $P_{t}$ is uniformly stochastically continuous in some neighborhood of $x=0$. The constant $\alpha$ is called the order of the function (or associated process).

Associated with such a transition function is the semi-group of operators $\left\{Q_{t}\right\}$ defined by

$$
Q_{t} f(x)=\int_{0}^{\infty} P_{t}(x, d y) f(y)
$$

for any bounded, measurable function $f$. For properties and facts about the semistable transition function, please refer to ([45], pg. 208-210).

Definition 3.2.2. A semi-stable Markov process (SSMP) is a strong Markov process with right-continuous paths having no discontinuities except jumps, whose transition probabilities are give by a semi-stable transition function.

For the rest of this chapter, $\left\{x_{t}\right\}$ will always denote a Markov process with a semi-stable transition function and with the "nice paths" ${ }^{2}$ guaranteed by Lemma 2.3 in [45]. We denote the space of right-continuous functions with left limits everywhere by $\Omega$; the Borel field generated by the cylinder sets by $\mathcal{F}$; and the probability measures of the process $\left\{x_{t}\right\}$, with $x_{0}=x$, by $P_{x}$. Let us now define the first passage time for the process $\left\{x_{t}\right\}$

$$
\xi=\inf \left\{t>0: x_{t}=0 \text { or } x_{t-}=0\right\}
$$

it is quite possible that $\xi=\infty$. If $\left\{x_{t}\right\}$ is strong Markov and so quasi-left continuous,

\footnotetext{
${ }^{2}$ That is, paths that are almost surely right-continuous for all $t$ and have no discontinuities other than jumps, and if $\left\{x_{t}\right\}$ is strong Markov, its paths are also quasi-continuous from the left.
} 
then $x_{t-}=0$ implies $x_{t}=0$, but this might not hold in general. In any event, as we have defined $\xi$ the events $\{\xi>t\}$ belong to the Borel field $\mathcal{F}$, and so the law of $\xi$ is determined by the transition function $P_{t}$. Now we prove a fact used often later:

Lemma 3.2.1. Either $P_{x}(\xi<\infty)=1$ for all $x>0$, or else $P_{x}(\xi<\infty)=0$ for all $x>0$.

Proof. The set $F=\left\{x_{t}: \exists \tau \in(0, \infty)\right.$ such that $x_{\tau}=0$ or $\left.x_{\tau-}=0\right\}$ belongs to $\mathcal{F}$. Also $F$ has the property that $x_{t} \in F$ implies $a^{-\alpha} x_{a t} \in F$ for all $a>0$. Then $P_{x}(F)$ is independent of $x, x>0$. We prove this assertion briefly before moving on. Since from the semi-stable condition (3.13), it is very easy to see that

$$
P_{x}(F)=P_{a^{\alpha} x}\left(\left\{x_{(\cdot)}: a^{-\alpha} x_{a t} \in F\right\}\right)
$$

for any cylinder set $F$. Then by the uniqueness of the extension of measure to $\mathcal{F}$, (3.16) must also hold for all sets $F \in \mathcal{F}$. But if $F$ satisfies the property that $x_{t} \in F$ implies $a^{-\alpha} x_{a t} \in F$ for all $a>0$, the right side of (3.16) equal $P_{a^{\alpha} x}(F)$. Since $a>0$ is arbitrary, then we have that $P_{x}(F)$ is independent of $x, x>0$.

$$
\begin{aligned}
& \text { Now, set } P_{x}(F)=p \text {. For any } t>0 \\
& P_{x}(t<\xi<\infty)=E_{x}\left(\chi_{\{t<\xi\}} P_{x}\left(x_{\tau}=0 \text { or } x_{\tau-}=0 \text { for some } \tau \in(t, \infty) \mid \mathcal{F}_{t}\right)\right)
\end{aligned}
$$

where $\chi_{A}$ is the indicator function of $A$ and $\mathcal{F}_{t}$ is the subfield of $\mathcal{F}$ generated by $\left\{x_{s} ; s \leq t\right\}$. However, by the (simple) Markov property, with probability one $\left(P_{x}\right)$ we have (when $x_{t}>0$ )

$$
P_{x}\left(x_{\tau}=0 \text { or } x_{\tau-}=0 \text { for some } \tau \in(t, \infty) \mid \mathcal{F}_{t}\right)=P_{x_{t}}(\xi<\infty)=p
$$


and so for all $t$

$$
P_{x}(t<\xi<\infty)=p P_{x}(t<\xi)
$$

But then

$$
p=P_{x}(\xi \leq t)+P_{x}(t<\xi<\infty)=P_{x}(\xi \leq t)+p P_{x}(t<\xi)
$$

so that $(1-p) P_{x}(\xi \leq t)=0$. Thus, unless $p=1$, we have $P_{x}(\xi \leq t)=0$ for all $x>0$ and all $t$ so that there is a.s. no zero; this proves the lemma.

Now, we define

$$
\phi_{\tau}(\omega)=\int_{0}^{\tau} x_{s}(\omega)^{-1 / \alpha} d s
$$

which is obviously a continuous and strictly increasing function of $\tau$ as long as $\tau<\xi$. In view of the quasi-left continuity of $\left\{x_{t}\right\}$, we now can use the simpler definition

$$
\xi=\min \left\{t: x_{t}=0\right\}
$$

Let $T(t)$ be the inverse function to $\phi$, or in other words, define the random variables $T(t)=T(t, \omega)$ as the (unique) solutions of the equation

$$
t=\int_{0}^{T} x_{s}(\omega)^{-1 / \alpha} d s=\phi_{T}
$$

which exist when $t<\phi_{\xi-}$. We assume $x_{0}=x>0$, so that $\xi>0$ a.s. Next, we define

$$
y_{t}(\omega)=x_{T(t, \omega)(\omega)},
$$

provided $t<\phi_{\xi-}$, and $y_{t}$ is not defined otherwise. According to a theorem first published by Volkonski ([26], Chapter 10) the random variables $\left\{y_{t}\right\}$ constitute a new strong Markov process whose paths again have the properties that they are 
right continuous and have no discontinuity other than jumps. We also note that $y_{t}>0$ as long as it is defined, so that we can set $z_{t}=\ln y_{t}$; clearly $\left\{z_{t}\right\}$ will be a strong Markov process on $(-\infty, \infty)$ with nice paths.

\subsubsection{The Theorem}

The following theorem relates semi-stable Markov processes to the well studied Lévy processes and is truly the heart of 1972 paper [45]. More importantly, it is this theorem that makes the idea of leveraging Lévy processes possible, which is the main focus of this thesis. Because the proof involves many other theorems and lemmas, we state the theorem without proof. The proof of this theorem can be found in [45] pg. 215-218.

Theorem 3.2.2. The process $z_{t}=\ln x_{T(t)}$ has stationary independent increments. The lifetime of $\left\{z_{t}\right\}$ is a.s. infinite either if $\left\{x_{t}\right\}$ does not reach 0 , or if it does so by continuous approach. If $\left\{x_{t}\right\}$ jumps to 0 , then $\left\{z_{t}\right\}$ may be considered to be an additive process with infinite lifetime which has been killed by an exponentially distributed random variable independent of the process.

We make a couple of remarks to emphasize the equations that relate semistable Markov processes (SSMP) to Lévy processes (or vise versa).

Remark 3.2.1. Writing $x_{t}$ (SSMP of order $\alpha$ ) and $z_{t}$ (Lévy) together in one equation is simply

$$
e^{z_{t}}=x\left(\int_{0}^{t}\left(e^{z_{s}}\right)^{\frac{1}{\alpha}} d s\right)
$$

Here, please forgive the change in notation from $x_{t}$ to $x(t)$, for the sake of clarity. 
To see why (3.24) is true, remember

$$
e^{z_{t}}=y_{t}:=x_{T(t)},
$$

and $T(t)$ is the inverse function to $\phi_{\tau}=\int_{0}^{\tau} x_{s}{ }^{-1 / \alpha} d s$ and is the unique solution to equation (3.22). Differentiating both sides of (3.22), we get

$$
d t=x_{T(t)}^{-1 / \alpha} d T(t)
$$

therefor

$$
d T(t)=x_{T(t)}^{1 / \alpha} d t
$$

hence,

$$
T(t)=\int_{0}^{t} x_{T(s)}^{1 / \alpha} d s
$$

Thus (3.25) then gives the relation

$$
\left.e^{z_{t}}=x\left(\int_{0}^{t} x_{T(s)}^{1 / \alpha}\right) d s\right)=x\left(\int_{0}^{t}\left(e^{z_{s}}\right)^{\frac{1}{\alpha}} d s\right) .
$$

Remark 3.2.2. Consider the case when the order $\alpha$ is one, and change notations to $\xi_{t}$ as the Lévy process; $L_{t}^{\left(\xi_{t}\right)}$ as SSMP (or Lamperti processes consistent with later use in Chapter 4), termed as the Lamperti associated with the Lévy. We have the so-called Lamperti Representation of equation (3.24) as

$$
e^{\xi_{t}}=L^{\left(\xi_{t}\right)}\left(\int_{0}^{t} e^{\xi_{s}} d s\right)
$$

Remark 3.2.3. In the paper by Jacobsen and Yor (2002, [39]), the concept of selfsimilar processes, together with the Lamperti Representation has been generalized to processes in $n$ dimensions. 
Remark 3.2.4. Suppose $e^{\xi_{t}}=L\left(A_{t}\right), A_{t}=\int_{0}^{t} e^{\xi(s)} d s=\int_{0}^{t} L(A(s)) d s$, and denote $\mathcal{L}$ the IG of $\xi_{t}$, and $L$ the IG of $L_{t}$, we have the relation between infinitesimal generators:

$$
\begin{aligned}
L f(l) & =\frac{1}{l} \mathcal{L}(f \circ \exp )(\ln l) \\
\mathcal{L} f(\xi) & =e^{\xi} L(f \circ \ln )\left(e^{\xi}\right) .
\end{aligned}
$$

Proof. Suppose $Y_{t}=L(A(t))=e^{\xi_{t}}$, and let $B$ be the generator of $\left\{Y_{t}\right\}$. It's easy to see that a function $f$ on $(0, \infty)$ is in the domain of $B$ iff $g(\xi)=f\left(e^{\xi}\right)$ is in the domain of $\mathcal{L}$, and that then $B f\left(e^{\xi}\right)=\mathcal{L} g(\xi)$. Also notice that $B=l L$ (obtained by random time substitution), so

$$
\begin{aligned}
l L f\left(e^{\xi}\right) & =B f\left(e^{\xi}\right)=\mathcal{L} g(\xi) \\
L f(l) & =\frac{1}{l} \mathcal{L}(f \circ \exp )(\ln l)
\end{aligned}
$$

The proof of the second equation similarly follows. With these relations, we can go back and forth between Lévy and Lamperti. The next two sections discuss this in more detail.

\subsubsection{The Diffusion Case}

We assume that the semi-stable process $\left\{x_{t}\right\}$ has (a.s.) trajectories which are continuous for all $t$. According to Theorem 3.2.2, the process $\left\{z_{t}\right\}$ has independent increments; it cannot have finite lifetime since $\left\{x_{t}\right\}$ does not jump to 0 . Since $\left\{x_{t}\right\}$ 
has continuous paths, so does $\left\{z_{t}\right\}$; it follows that $\left\{z_{t}\right\}$ can be nothing other than Brownian motion, possibly degenerate and possibly with a constant drift superimposed. In the degenerate case, $\left\{z_{t}\right\}$ is the deterministic motion $z_{t}=b t+z_{0}$. It is not hard to reverse the transformations embodied in Theorem 3.2 .2 in this case. Specifically

$$
\phi(t)=\int_{0}^{t} x(s)^{-1 / \alpha} d s
$$

and $z_{\phi(t)}=\ln x_{T(\phi(t))}=\ln x_{t}$, so then

$$
z_{\phi(t)}=b \int_{0}^{t} x(s)^{-1 / \alpha} d s+z_{0}=\ln x_{t}
$$

or

$$
\int_{0}^{t} x(s)^{-1 / \alpha} d s=\frac{1}{b}\left(\ln x_{t}-z_{0}\right)
$$

and the result is

$$
x_{t}=\left(x_{0}^{1 / \alpha}+\frac{b t}{\alpha}\right)^{\alpha}
$$

as long as $x_{t}>0$, where $\alpha>0$ is the order of the process and $x_{0}=e^{z_{0}}>0$. In case $b<0$, after reaching 0 the process can only be continued by sticking at 0 if path continuity is to be maintained. When $b>0$ and $x_{0}=0$, either $x_{t} \equiv 0$ or $x_{t}=(b t / \alpha)^{\alpha}$ are possible choices for completing the definition of $\left\{x_{t}\right\}$.

The description of $\left\{x_{t}\right\}$ in all other cases is stated in the following theorem:

Theorem 3.2.3. Any non-degenerate semi-stable Markov process on $\mathbb{R}^{+}$with (a.s.) 
continuous paths has a generator of the form

$$
A f(x)=b x^{1-1 / \alpha} f^{\prime}(x)+d x^{2-1 / \alpha} f^{\prime \prime}(x), \quad x>0
$$

where $d>0$ and $b$ are constants.

Proof. For the process $\left\{x_{t}\right\}$ considered here the corresponding additive process $\left\{z_{t}\right\}$ must be simply a Wiener process with a constant drift. Thus the generator $C$ of $\left\{z_{t}\right\}$ is a differential operator with constant coefficients

$$
C g(z)=c g^{\prime}(z)+d g^{\prime \prime}(z),
$$

$d>0$ since the deterministic case is now excluded.

Let us now return to $(0, \infty)$ via the transformation $y_{t}=\exp \left(z_{t}\right)$, and let $B$ denote the generator of $\left\{y_{t}\right\}$. It is easy to see that a function $f$ on $(0, \infty)$ is in the domain of $B$ if and only if $g(z)=f\left(e^{z}\right)$ is in the domain of $C$, so that $B f\left(e^{z}\right)=C g(z)$. We change the variable by letting $e^{z}=x$, which implies that

$$
g^{\prime}(z)=f^{\prime}\left(e^{z}\right) e^{z}=f^{\prime}(x) x
$$

and

$$
g^{\prime \prime}(z)=f^{\prime \prime}\left(e^{z}\right) e^{2 z}+f^{\prime}\left(e^{z}\right) e^{z}=f^{\prime \prime}(x) x^{2}+f^{\prime}(x) x .
$$

Thus

$$
\begin{aligned}
B f(x) & =B f\left(e^{z}\right)=C g(z) \\
& =c g^{\prime}(z)+d g^{\prime \prime}(z) \\
& =c f^{\prime}(x) x+d\left(f^{\prime \prime}(x) x^{2}+f^{\prime}(x) x\right) \\
& =(c+d) x f^{\prime}(x)+d x^{2} f^{\prime \prime}(x) .
\end{aligned}
$$


Eventually, we come back to $\left\{x_{t}\right\}$. Since the relation $B=x^{1 / \alpha} A$ holds between the generator $A$ of $\left\{x_{t}\right\}$ and that of the process $\left\{y_{t}\right\}$ obtained by random time substitution, (3.30) follows at once with $b:=c+d$.

\subsubsection{The generator of a SSMP}

Let us now generalize Theorem 3.2.3 to obtain a formula for the generator of any SSMP on $\mathbb{R}^{+}$when $x>0$. Let $\left\{x_{t}\right\}$ be an SSMP of order $\alpha$, and let $\left\{z_{t}\right\}$ be that additive process which corresponds to $\left\{x_{t}\right\}$ according to Theorem 3.2.2. By the theorem of Lévy and Khintchine, we have

$$
E\left(e^{i \lambda\left(z_{t}-z_{0}\right)}\right)=\exp \left\{i \mu t \lambda+t \int_{-\infty}^{\infty}\left(e^{i \lambda y}-1-\frac{i \lambda y}{1+y^{2}}\right) \frac{1+y^{2}}{y^{2}} d G(y)\right\}
$$

where $d G$ is a finite measure uniquely determined by $\left\{z_{t}\right\}$. According to a theorem due to Ito and Neveu (see [48], pg. 628-630), the generator $C$ of $\left\{z_{t}\right\}$ has the form

$$
C g(z)=\mu g^{\prime}(z)+\int_{-\infty}^{\infty} h(z, y) d G(y)
$$

where

$$
h(z, y)=\left\{\begin{array}{cc}
{\left[g(z+y)-g(z)-\frac{y}{1+y^{2}} g^{\prime}(z)\right] \frac{1+y^{2}}{y^{2}}} & \text { for } y \neq 0 \\
g^{\prime \prime}(z) / 2 & \text { for } y=0
\end{array}\right.
$$

The domain of $C$ contains at least all functions $g$ such that $g, g^{\prime}$ and $g^{\prime \prime}$ are continuous on $[-\infty, \infty]$. 
Theorem 3.2.4. Let $\left\{x_{t}\right\}$ be a SSMP of order $\alpha$, whose corresponding additive process $\left\{z_{t}\right\}$ satisfies (3.32). Then the characteristic operator of $\left\{x_{t}\right\}$ has for $x>0$ the form

$$
\mathcal{A} f(x)=\mu x^{1-1 / \alpha} f^{\prime}(x)+x^{-1 / \alpha} \int_{0}^{\infty} h^{*}(x, u) d G^{*}(u)-\beta x^{-1 / \alpha} f(x),
$$

where $G^{*}(u)=G(\ln u)$ and

$$
h^{*}(x, u)=\left\{\begin{array}{cl}
{\left[f(x u)-f(x)-\frac{\ln u}{1+(\ln u)^{2}} x f^{\prime}(x)\right] \frac{1+(\ln u)^{2}}{\ln u^{2}}} & \text { for } u \neq 1 \\
x^{2} f^{\prime \prime}(x) / 2 & \text { for } u=1
\end{array}\right.
$$

Formula (3.35) holds at least for all $f$ such that $f, x f^{\prime}$ and $x^{2} f^{\prime \prime}$ are continuous on $[0, \infty]$, and uniquely determines the process $\left\{x_{t}\right\}$ for all $t<\xi$. Conversely, give $\left\{x_{t}\right\}, \mu, G^{*}$ and $\beta$ are determined.

Proof. Formulas (3.35) and (3.36) follow formally from (3.33) and (3.34) in exactly the same way that (3.30) was derived from (3.31); indeed, the latter is a special case. Since $g \in \mathcal{D}_{C}$ when $g, g^{\prime}, g^{\prime \prime}$ are continuous on $[-\infty, \infty]$, we find that $f(x)=g(\ln x) \in \mathcal{D}_{B}\left(B\right.$ being the generator of $\left.\left\{y_{t}\right\}=\left\{e^{z_{t}}\right\}\right)$ when $f, x f^{\prime}$ and $x^{2} f^{\prime \prime}$ are continuous on $[0, \infty]$. For at least these functions, therefore, the characteristic operator of $\left\{x_{t}\right\}$ is given by $\mathcal{A}=x^{-1 / \alpha} B$. But the formula for $B f$ is obtained from (3.33) and (3.34) by a change of variable; combining the result with $\mathcal{A}=x^{-1 / \alpha} B$ leads to (3.35) and (3.36). 


\subsubsection{Bessel Squared Processes}

Bessel processes possess two major features: first, besides the Ornstein-Uhlenbeck processes, they are essentially the only diffusion processes in addition to Brownian motion (with drift), for which a relatively simple expression for the transition probability is known; second, they appear naturally in a number of interesting problems in finance and insurance. For instance, hypergeometric functions, which are related to Bessel processes, are used for the pricing of options on zero coupon bonds in the Cox-Ingersoll-Ross general equilibrium model of interest rates. Another key point is that the standard hypothesis in most financial papers assumes stock price dynamics driven by the exponential of a Brownian motion with drift, which is in turn the square of a time-changed Bessel process. Especially, this reminds us that Bessel squared processes, BESQ, plays one of the two roles of the bijection between exponentials of Lévy processes (in this case, the diffusion case, standard Brownian motion) and semi-stable Markov processes introduced by Lamperti. In fact, the family of BESQ processes is the only family of continuous semi-stable Markov processes.

Let us now introduce the Bessel squared process of dimension $\delta, \mathrm{BESQ}^{\delta}$. A Bessel process BES is the square root of a BESQ.

Definition 3.2.3. For any $\delta \geq 0$, the $\delta$-dimensional Bessel squared process BESQ $\delta$ is a continuous diffusion process $Z_{t}^{\delta}=\left(Z^{\delta}(t), t \geq 0\right)$ taking its values in $\mathbb{R}_{+}$and satisfying the stochastic differential equation

$$
d Z_{t}^{\delta}=\delta d t+2 \sqrt{Z_{t}^{\delta}} d W_{t}, \quad Z_{0}=z_{0} \geq 0
$$


or the stochastic integral form

$$
Z_{t}^{\delta}=z_{0}+\delta t+2 \int_{0}^{t} \sqrt{Z_{s}^{\delta}} d W_{s}
$$

where $W_{t}$ is a standard one-dimensional Brownian motion.

When $\delta \geq 2, \mathrm{BESQ}^{\delta}$ will never reach 0 for $t>0$; for $0 \leq \delta<2, \mathrm{BESQ}^{\delta}$ will reach 0 a.s.; when $\delta<0, \mathrm{BESQ}^{\delta}$ hits 0 a.s. The real $\nu=\frac{\delta}{2}-1$ is called the index of the process $\mathrm{BESQ}^{\delta}$. As a consequence of the stochastic differential equation satisfied by $\left(Z^{\delta}(t), t \geq 0\right)$, the process

$$
\left\{f\left(Z_{t}^{\delta}\right)-\int_{0}^{t}\left(I^{\delta} f\right)\left(Z_{s}^{\delta}\right) d s, t \geq 0\right\}
$$

is a local martingale as long as $f$ belongs to $C^{2}((0, \infty))$, where

$$
I^{\delta} f(z)=2 z \frac{d^{2} f}{d z^{2}}+\delta \frac{d f}{d z}
$$

and $I^{\delta}$ is the infinitesimal generator associated with $\mathrm{BESQ}^{\delta}$.

We denote by $Q_{z_{0}}^{\delta}$ the distribution of the process $\mathrm{BESQ}^{\delta}$ starting at $z_{0} \geq 0$; this distribution is defined on the canonical space of continuous functions $C\left(\mathbb{R}_{+}, \mathbb{R}_{+}\right)$on which we consider the coordinate process $(R(t), t \geq 0)$ which is defined by $R_{t}(f)=$ $f(t)$ for every $f \in C\left(\mathbb{R}_{+}, \mathbb{R}_{+}\right)$and the $\sigma$-field $\mathcal{G}=\sigma\left(R_{t}, t \geq 0\right)$.

The following important property of the Bessel squared processes was obtained by Shiga and Watanabe (1973, [63]).

Proposition 3.2.5. For every $\delta, \delta^{\prime}, x, x^{\prime} \geq 0, Q_{x}^{\delta} \otimes Q_{x^{\prime}}^{\delta^{\prime}}=Q_{x+x^{\prime}}^{\delta+\delta^{\prime}}$, where $P \otimes Q$ denotes the distribution of the process $\left(X_{t}+Y_{t}, t \geq 0\right)$, for $X_{t}$ and $Y_{t}$, two independent processes with respective distributions $P$ and $Q$. 
This property permits, whether $\delta$ is an integer or not, the reduction of a number of problems involving $\mathrm{BESQ}^{\delta}$ to the case $\delta=1$, where $\mathrm{BESQ}^{1}$ is precisely the square of one-dimensional Brownian motion.

Recall that if $W_{t}^{x}$ is a Brownian motion started at $x$, then for any $c>0$, the processes $W_{c^{2} t}^{x}$ and $c B_{t}^{x / c}$ have the same law.

Proposition 3.2.6. If $Z$ is a $B E S Q^{\delta}$ started at $z$ and $c>0$, then $Z_{c t} / c$ is a $B E S Q^{\delta}$ started at $z / c$.

Proposition 3.2.6 can be easily proved by a change of variable in the stochastic integral in 3.37

$$
Z_{c t} / c=z_{0} / c+\delta t+2 \int_{0}^{t} \sqrt{Z_{c s} / c} d\left(W_{c s} / \sqrt{c}\right)
$$

and $W_{c s} / \sqrt{c}$ is a Brownian motion.

Remark 3.2.5. We should realize here Proposition 3.2.6 implies that $\left\{Z_{c t}\right\} \approx\left\{c Z_{t}\right\}$. Hence $Z_{t}$ is a semi-stable Markov process of order $\alpha=1$.

Bessel (squared) processes are, by definition, Markov processes and their transition functions have the following form.

Proposition 3.2.7. For $\delta>0$, the semigroup of $B E S Q^{\delta}$ has a density in y equal to

$$
q_{t}^{\delta}(x, y)=\frac{1}{2 t}\left(\frac{y}{x}\right)^{\nu / 2} \exp \left(-\frac{x+y}{2 t}\right) I_{\nu}\left(\frac{\sqrt{x y}}{t}\right), \quad t>0, x, y>0
$$

where $I_{\nu}$ is the modified Bessel function of first kind with index $\nu$.

The density of the semigroup of the Bessel process $\mathrm{BES}^{\delta}$ can be obtained from (3.38) by a straightforward change of variable and is found equal, for $\delta>0$, to

$$
p_{t}^{\delta}(x, y)=\frac{1}{t}\left(\frac{y}{x}\right)^{\nu} y \exp \left(-\frac{x^{2}+y^{2}}{2 t}\right) I_{\nu}\left(\frac{x y}{t}\right), \quad t>0, x, y>0,
$$


The key result for our applications is due to Williams (1974, [70]).

Proposition 3.2.8. The exponential of Brownian motion with drift is a timechanged Bessel process; more specifically,

$$
\exp (W(t)+\nu t)=R^{(\nu)}\left(\int_{0}^{t} \exp 2(W(s)+\nu s) d s\right), \quad t \geq 0
$$

where $\left(R^{(\nu)}(u), u \geq 0\right)$ is a Bessel process with index $\nu$.

We can give two extensions of Proposition 3.2.8.

1. A similar result holds more generally for $(\exp (a W(t)+\nu t), t \geq 0)$. Thanks to the scaling property of Brownian motion, we can write

$$
\begin{aligned}
\exp (a W(t)+\nu t) & =\exp \left(\hat{W}\left(t a^{2}\right)+\frac{\nu}{a^{2}} t a^{2}\right. \\
& =\hat{R}^{\left(\nu / a^{2}\right)}\left(\int_{0}^{t a^{2}} \exp 2\left(\hat{W}(s)+\frac{\nu s}{a^{2}}\right) d s\right) \\
& =\hat{R}^{\left(\nu / a^{2}\right)}\left(a^{2} \int_{0}^{t} \exp 2\left(\hat{W}\left(a^{2} u\right)+\nu u\right) d u\right)
\end{aligned}
$$

(with $\hat{W}$ denoting another Brownian motion, and corresponding $\hat{R}$ from Proposition 3.2.8) so that

$$
\exp (a W(t)+\nu t)=\hat{R}^{\left(\nu / a^{2}\right)}\left(a^{2} \int_{0}^{t} \exp 2(a W(u)+\nu u) d u\right)
$$

2. The exponential of Brownian motion with drift is a time-changed Bessel squared process. This property is straightforward since, from (3.41), we deduce 


$$
\begin{aligned}
\exp (a W(t)+\nu t) & =\exp 2\left(\frac{a}{2} W(t)+\frac{\nu t}{2}\right) \\
& =\left[\hat{R}^{\left(2 \nu / a^{2}\right)}\left(\frac{a^{2}}{4} \int_{0}^{t} \exp 2\left(\frac{a}{2} W(s)+\frac{\nu}{2} s\right) d s\right)\right]^{2}
\end{aligned}
$$

There are a couple of important transformations which relate the squared Bessel processes with other well known processes. We here look at three examples which start with the CIR (Cox-Ingersoll-Ross) process.

1. The Cox-Ingersoll-Ross (CIR) processes

$$
X_{t}=x+\int_{0}^{t}\left(a+b X_{s}\right) d s+\sigma \int_{0}^{t} \sqrt{X_{s}} d W_{s}
$$

for $t \geq 0, a \geq 0, \sigma \geq 0$, were introduced in $([20])$ as in a model for interest rates. The CIR process is a space-time transformed BESQ process, more explicitly: A $\mathrm{BESQ}^{\delta}$ process $Z$ can be transformed to the CIR process $X$ by

$$
X_{t}=e^{b t} Z\left(\frac{\sigma^{2}}{4 b}\left(1-e^{-b t}\right)\right)
$$

where $\delta=4 a / \sigma^{2}$.

2. A squared radial Ornstein-Uhlenbeck process is the solution to the equation

$$
Y_{t}=y+\int_{0}^{t}\left(\delta-2 \lambda Y_{s}\right) d s+2 \int_{0}^{t} \sqrt{Y_{s}} d W_{s}
$$

for $t \geq 0, \delta \geq 0$. A squared radial Ornstein-Uhlenbeck process can be transformed by the time transformation $g(t)=\sigma^{2} t / 4$ to the CIR process $X$, where $\delta=4 a / \sigma^{2}$, $\lambda=-2 b / \sigma^{2}$. Hence it can also be related to squared Bessel process. 
3. Consider the stock price process $S_{t}$ has independent increments with constant elasticity of variance. That is:

$$
d S_{t}=\mu S_{t} d t+\sigma S_{t}^{\rho} d W_{t}, \quad S_{0}=s \geq 0
$$

where $\rho$ means the elasticity of variance. This model was first considered by Cox ([19]) where it was called the constant elasticity of variance model (CEV model). For $\rho<1$ is a well known model that many have studied. Here we draw on Delbaen and Shirakawa (1996, [22]). We may define the BESQ process of dimension $\delta$ by $X^{(\delta)}$ with the SDE

$$
d X_{t}^{(\delta)}=\delta d t+2 \sqrt{X_{t}^{(\delta)}} d W_{t}
$$

For the parameters $\nu>0$ and $\delta<2$ consider the deterministic time change

$$
\tau_{t}^{(\delta, \nu)}=\frac{\sigma^{2}}{2 \nu(2-\delta)}\left(1-\exp \left(-\frac{2 \nu}{2-\delta} t\right)\right)
$$

then define the process

$$
Y_{t}^{\delta, \nu}=e^{\nu t}\left(X_{\tau_{t}^{(\delta, \nu)} \wedge \zeta}^{(\delta)}\right)^{1-\frac{\delta}{2}}
$$

where

$$
\zeta=\inf \left\{t \mid X_{t}^{(\delta)}=0\right\}
$$

We have the result that

$$
\left\{S_{t}, t \geq 0\right\} \stackrel{\text { law }}{=}\left\{Y_{t}^{\left(\delta_{\rho}, \mu\right)}, t \geq 0\right\}
$$


where

$$
\delta_{\rho}=\frac{1-2 \rho}{1-\rho}
$$

Hence such price processes are deterministic time change of BESQ raised to a power where the dimension relates to the power $\rho$ as does the power of the BESQ which is

$$
\frac{1}{2(1-\rho)}
$$

We also know that $X_{t}^{(\delta)}$ is the Lamperti process (or $S S M P$ ) for the Lévy process

$$
2 W_{t}+(\delta-2) t
$$

and this relates the solution of CEV to Lamperti for

$$
2 W_{t}-\frac{1}{1-\rho} t
$$

\subsection{Exponential Functionals of Brownian Motion and Related Pro- cesses}

In computing the price of Asian options, the study of the variable $\int_{0}^{t} S(u) d u$ comes to interest. We assume the asset price driven under the risk-neutral probability measure $Q$ by the dynamics described as

$$
d S(t)=r S(t) d t+\sigma S(t) d W(t)
$$

We also assume that the number of values whose average is computed is large enough 
to allow the representation of the average $A(T)$ over $[0, T]$ by the integral

$$
A(T)=\frac{1}{T} \int_{0}^{T} S(u) d u
$$

This quantity is needed for calculating the option price, say call option, as in

$$
C_{t, T}(K)=e^{-r(T-t)} E_{Q}\left[(A(T)-K)^{+} \mid \mathcal{F}_{t}\right]
$$

In general we are interested in computing integral

$$
\int_{0}^{t} \exp (a s+\sigma W(s)) d s
$$

where here $a$ may be equal to $r-\frac{1}{2} \sigma^{2}$. This exponential function of Brownian motion has recently been a subject of common interest for mathematicians and for physicists. The Lamperti Representation stated in Proposition 3.2.8, and more specifically in equation (3.41), exhibits the importance of the functional 3.44. After the study of of Lamperti's 1972 paper $([45])$, we now know that this representation is a particular instance of the Lamperti relation which expresses $\left(\exp \left(\xi_{t}\right), t \geq 0\right)$ as

$$
e^{\xi_{t}}=X\left(\int_{0}^{t} e^{2 \xi_{s}} d s\right)
$$

where $\left(\xi_{t}, t \geq 0\right)$ is a Lévy process and $(X(u), u \geq 0)$ is a semi-stable Markov process.

In the article by Carmona, Petit and Yor (1994, [71]), they generalize the work of M. Yor concerning the law of $A=\int_{0}^{T} \exp \left(\xi_{s}\right) d s$ where $\xi$ is a Brownian motion with drift and $T$ an independent exponential time, to the case where $\xi$ belongs to a certain class of Lévy processes. Their method again hinges on a bijection, introduced by Lamperti, between exponentials of Lévy processes and semi-stable 
Markov processes. A collection of articles dealing with the exponential functionals of Brownian motion and related processes during 1992 and 1998, is in a book by Marc Yor $([72])$.

In this chapter, we introduce the semi-stable stochastic processes as limits upon subjecting a fixed stochastic process to infinite contractions of its time and space scales. We also establish the lamperti relation which connects semi-stable Markov processes to Lévy processes. This important relation will be put into use as we attempt to build leverage into Lévy processes as models for stock prices. Chapter 4 starts with the general set up and from then on our study of leveraging Lévy processes via Lamperti has begun. 


\section{Chapter 4}

\section{LEVERAGING LÉVY VIA LAMPERTI}

\subsection{Leveraging Lévy}

The leverage effect is where negative return sequences are associated with increases in the volatility of stock returns. The leverage effect was studied in some early work by Black $(1976,[8])$, while it motivated the introduction of the EGARCH model of Nelson (1991, [52]) and the threshold ARCH model of Glosten, Jagannathan, and Runkle (1993, [31]). An economic theory behind such effects is dis cussed by Campbell and Kyle (1993, [10]).

Models for the stock price process typically arrange for the limited liability feature and attain positivity of the price process by explicitly modeling the logarithm of the price. The literature now contains many examples of log prices modeled as Lévy processes. These processes are processes of independent and identically distributed increments with constant volatilities and as a consequence are devoid of a leverage effect. To incorporate leverage into these models we follow the local Lévy development of Carr, Geman, Madan and Yor (2003, [13]) by introducing a time change that depends on the level of the asset price. This dependence affects the expected local quadratic variation in an explicit manner and hence builds in desired levels of leverage. We begin now with the general model to set a foundation for understanding how semi-stable Markov processes [45] come into play. 
From now on, we will always term semi-stable Markov processes as Lamperti processes associated with some Lévy processes, or in short Lamperti processes.

\subsection{The General Model}

\subsubsection{Leveraging Lévy}

Let the stock price process be denoted by $S_{t}=(S(t), t \geq 0)$ and let $X_{t}=$ $(X(t), t \geq 0)$ be the $\log$ price process with $X_{t}=\ln S_{t}$. Additionally let $\xi_{t}=(\xi(t), t \geq$ 0) be a Lévy process that we wish to see leveraged. To begin with, we focus our attention on the case of constant elasticity of variance and define the desired local speed adjustment $A\left(S_{t}\right)$, at spot price $S$, for the lévy process as $A\left(S_{t}\right)=S_{t}{ }^{a}$.

It follows that the desired model for the log price is the following time changed Lévy process,

$$
X(t)=\xi\left(\int_{0}^{t} S(u)^{a} d u\right)=\xi\left(\int_{0}^{t} e^{a X(u)} d u\right)
$$

Here the specific time change for the Lévy process is defined as $T_{t}=(T(t), t \geq 0)$ where

$$
T(t)=\int_{0}^{t} e^{a X(u)} d u
$$

We note that if $(k(x), x \in \mathbb{R})$ is the Lévy density for the Lévy process $\xi$ then the process $X(t)$ has a Lévy system with jump compensation measure $\nu_{X}(d x, d u)$ given by

$$
\nu_{X}(d x, d u)=e^{a X(u)} k(x) d x d u=S(u)^{a} k(x) d x d u
$$

and the compensator splits into a product of two functions. The first depends on 
the asset price and incorporates leverage while the second addresses the specific jump sizes. For the expected negative relation between volatility and spot price, we expect to estimate the parameter $a$ to be less than unity.

The risk neutral dynamics for the stock price are now given by

$d S(t)=(r-l) S(t-) d t+\sigma(S(t-), t) d W(t)+\int_{-\infty}^{\infty} S(t-)\left(e^{x}-1\right)\left(m(d x, d u)-\nu_{S}(d x, d u)\right)$ where $m(d x, d u)$ is the counting measure associated with the jumps in the logarithm of the stock price.

We term the class of processes $S_{t}$ for which $X_{t}=\ln S_{t}$ is defined by (4.1) as a $\left(\xi_{t}, a\right)$-Leveraged Lévy processes in recognition of specific space dependence of the Lévy system observed in (4.2). Leveraging Lévy processes are defined with reference to the specific form of the time change employed and they may or may not be martingales. When modeling the historical or true process, martingale restrictions do not arise. From a risk neutral perspective, however, we are interested in asset price processes being discounted martingales. We may accommodate this requirement by writing a model directly for the forward price as a martingale with leverage depending on the level of the forward price. The spot price is then defined as the discounted forward price. To get the forward price to be a martingale we choose the Lévy process such that its exponential is a martingale. The incorporation of our suggested time change leaves it a martingale. 


\subsubsection{Lamperti and Leveraged Lévy}

The class of Leveraged Lévy processes is closely tied to the processes studied by Lamperti [45] and we determine the laws of the process $X_{t}$ by relating the leveraged Lévy processes to the Lamperti processes. We first review how Lamperti processes are defined and the relation between a Lamperti process (SSMP, details in Chapter 4) and a Lévy process.

Given any one-dimensional Lévy process $\xi=\xi_{t}=(\xi(t), t \geq 0)$, Lamperti associated with such a Lévy process a positive Markov process that we denote by $L_{t}^{(\xi)}=\left(L_{t}^{(\xi)}, t \geq 0\right)$ and call it the Lamperti process associated with $\xi$. The process $L^{(\xi)}$ is implicitly defined by the time change

$$
\tau_{t}^{(\xi)}=\int_{0}^{t} e^{\xi(s)} d s
$$

whereby $L^{(\xi)}$ is defined as satisfying

$$
e^{\xi(t)}=L^{(\xi)}\left(\tau_{t}^{(\xi)}\right)=L^{(\xi)}\left(\int_{0}^{t} e^{\xi(s)} d s\right)
$$

One may deduce from the independence and stationarity of $\xi$ that $L^{(\xi)}$ satisfies the scaling property

$$
\left(L_{a t}^{(\xi)}, 0 \leq t ; P_{x}\right)=\left(a L_{t}^{(\xi)}, t \geq 0 ; P_{x / a}\right)
$$

where $P_{x}$ is the law of $L^{(\xi)}$ starting at $x$. In [45] Lamperti calls such positive processes as semi-stable Markov processes and then shows that there is a one-to-one correspondence between such semi-stable processes and Lévy processes via equation (4.3). This is precisely what was introduced in Section 3.2.2. 
This result leads to the following theorem.

Theorem 4.2.1. Every $\left(\xi_{t}, a\right)$-Leveraged Lévy processes $\left\{S_{t}\right\}$ is a Lamperti process raised to a power. Specifically,

$$
S_{t}=\left[L_{t}^{(-a \xi)}\right]^{(-1 / a)}
$$

Proof. The relationship between Lamperti processes and leveraged Lévy processes follows on considering the exponential of $a X(t)$ for a leveraged Lévy process $S(t)$. From equation (4.1) we see that

$$
\exp (a X(t))=\exp \left(a \xi\left(\int_{0}^{t} e^{a X(u)} d u\right)\right)
$$

Defining

$$
Z(t)=\exp (a X(t))
$$

we deduce that

$$
Z(t)=\exp \left(a \xi\left(\int_{0}^{t} Z(u) d u\right)\right)
$$

By defining the inverse time change $\zeta(t)$ of $T(t)$ by $T(\zeta(t))=t$, precisely

$$
\int_{0}^{\zeta(t)} e^{a X(u)} d u=t
$$

and evaluating both (4.6) and (4.7) at $\zeta(t)$, we obtain

$$
Z(\zeta(t))=\exp (a X(\zeta(t)))
$$

and

$$
Z(\zeta(t))=\exp (a \xi(t))
$$


hence,

$$
\exp (a \xi(t))=\exp (a X(\zeta(t))) .
$$

The time change $\zeta(t)$ is identified by differentiating (4.8) to obtain

$$
d t=e^{a X(\zeta(t))} d \zeta(t)=e^{a \xi(t)} d \zeta(t),
$$

or equivalently

$$
d \zeta(t)=e^{-a \xi(t)} d(t) .
$$

It follows that

$$
\zeta(t)=\int_{0}^{t} e^{-a \xi(s)} d(s)
$$

From (4.10) we have

$$
\exp (-a \xi(t))=Z^{-1}\left(\int_{0}^{t} e^{-a \xi(s)} d s\right)
$$

and we observe $Z^{-1}$ is the Lamperti process $L^{-a \xi}$ associated with $-a \xi(t)$.

But as $Z(t)=\exp (a X(t))$ we have that

$$
X(t)=\frac{1}{a} \ln (Z(t))=-\frac{1}{a} \ln \left(L^{-a \xi}\right),
$$

recalling that $S(t)=\exp (X(t))$ we see (4.4) holds.

Given the close connection between leveraged Lévy processes and the Lamperti processes, we review the connections between the infinitesimal generator of the Lévy process and that for the associated Lamperti process. Denote by $A$ the infinitesimal 
generator for the Lamperti process associated with a Lévy process $\xi$ and let $B$ denote the infinitesimal generator for $\xi$. We have the following relations [71]:

$$
\begin{aligned}
A f(l) & =\frac{1}{l} B(f \circ \exp )(\ln l), \\
B f(\xi) & \left.=e^{\xi} A(f \circ \ln )\right)\left(e^{\xi}\right) .
\end{aligned}
$$

We shall use this relations to go back and forth between Lévy processes and the associated Lamperti processes.

\subsection{Stock Price Modeling Using Leveraged Lévy Processes}

The model for the price of a stock suggested by leverage considerations in the context of local uncertainties characterized by Lévy processes in light of Theorem 4.2.1, is to employ powers of Lamperti processes. The specific power is related to the Lévy process used in constructing the Lamperti process.

From the perspective of risk neutral modeling we may be interested in either martingale models consistent with particular forms of no dynamic arbitrage, or if we focus attention on just the absence of static arbitrage following [13], we may wish to just impose particular levels for the term structure of forward prices as determined by market data. We term the former class of models, martingale leveraged Lévy models, while the latter are referred to as normalized leveraged Lévy models.

We present in several subsections the procedure for constructing both classes of models, given the prior choice of the Lévy process $\xi$ and the leverage coefficient $a$. 


\subsubsection{Martingale Leveraged Lévy Models}

Given a Lévy process $\xi$, consider a stock price model in the suggested form

$$
S(t)=\left[L^{(\xi / \alpha)}\right]^{\alpha}(t)
$$

where we have $\alpha=-\frac{1}{a}$. For a martingale model we need this stock price process to be a martingale. Note from equation (4.13) that

$$
\exp (-a \xi(t))=\left[L^{(\xi / \alpha)}\right]\left(\int_{0}^{t} e^{-a \xi(s)} d s\right)=\left[L^{(\xi / \alpha)}\right](\zeta(t))
$$

Evaluating this expression at the inverse of $\zeta, T(t)$ we get

$$
\exp (-a \xi(T(t)))=\left[L^{(\xi / \alpha)}\right](t)
$$

and by raising both sides to the power $\alpha$ we obtain

$$
\left[L^{(\xi / \alpha)}\right]^{\alpha}=\exp (\xi(T(t)))
$$

Recall that the original stock price model in Theorem 4.2.1 is $S(t)=\left[L_{t}^{(-a \xi)}\right]^{(-1 / a)}=$ $\left[L^{(\xi / \alpha)}\right]^{\alpha}$. So our original stock price model is now written as the exponential of a time changed Lévy process,

$$
S(t)=\exp (\xi(T(t)))
$$

To obtain a martingale process for the stock price we only have to choose the original Lévy process such that its exponential is a martingale. This may be easily accomplished by an adjustment of the drift term.

On the other hand, if it is discounted stock prices that are to be martingales then for a continuously compounded interest rate of $r$ and a dividend yield of $q$ we 
suggest the model

$$
S(t) e^{-(r-q) t}=\left[L^{(\xi / \alpha)}\right]^{\alpha}(t)
$$

Leverage is here formulated homogeneously in terms of the forward price, with the appropriate inhomogeneity when working with the asset spot price, but the essential dependence has been incorporated.

\subsubsection{Normalized Leveraged Lévy Models}

For particular Lévy processes that one associates with a Lamperti process it may be difficult to identify the laws of the Lamperti process after one has made the drift correction in the Lévy process to get a martingale as discussed in the last section. One may still be interested in models based on a leveraged Lévy process that is consistent with the absence of static arbitrage as studied in [13]. The approach here is to normalize a power of the Lamperti process to unit expectation and adjust the spot price to re-price correctly the forward contracts.

Specifically, let $L_{t}=(L(t), t \geq 0)$ be a Lamperti process. We define the stock price process by

$$
S(t)=S(0) e^{(r-q) t} \frac{L(t)^{\alpha}}{E\left[L(t)^{\alpha}\right]}
$$

The Lamperti process here may be obtained from a specific Lévy process or alternatively we may start with a Lamperti process from which we may construct a Lévy process $\xi$ that is consistent with it through the Lamperti equation (4.3). Taking the Lévy process as $\xi / \alpha$ or equivalently $-a \xi$, we recover the Lamperti as $L^{(-a \xi)}$ and 
thus

$$
\frac{S(t) e^{-(r-q) t} E\left[L(t)^{\alpha}\right]}{S(0)}=L(t)^{\alpha}
$$

is a $(\xi, a)$-leveraged Lévy process. We observe that a deterministic perturbation of the stock price is a leveraged Lévy process and so the stock price would inherit these leverage properties.

\subsubsection{The Absolute Value of a Cauchy}

We show one example of exact solutions for leveraged Lévy processes. Our approach is to begin with equation (4.16), recognizing that we need only to consider the power of a Lamperti process for the stock price process. The specific Lévy measure may then be deduced by application of (4.15). For a start we concern ourselves only with the absence of static arbitrage and develop processes for the stock price that when mean corrected, yield a measure on the space of paths that we employ for pricing.

We begin with any Lamperti process, which is any positive Markov process that satisfies the scaling property. A classic example of a Lamperti process is the absolute value of a Cauchy process ${ }^{1}$. Let $(C(u), u \geq 0)$ be the value of a Cauchy process and define by

$$
S(t)=S(0) e^{(r-q) t} \frac{|C(t)|^{-\beta}}{E\left[|C(t)|^{-\beta}\right]} .
$$

\footnotetext{
${ }^{1}$ Recall that the Cauchy process is a one-dimensional Brownian motion with random time evolution as the Lévy subordinator.
} 
where we take $\beta>1$ and hence as $a=1 / \beta$, we have $0<a<1$. This process allows for an elastic leverage effect. We organize $S(t)$ to approach $S(0)$ as $t$ tends to 0 by starting $C(t)$ at 1 . Defining

$$
\omega(t)=-\ln \left(E\left[|C(t)|^{-\beta}\right]\right)
$$

we may then write $\ln S(t)$ as

$$
\ln S(t)=\ln S(0)+(r-q) t+\omega(t)-\beta \ln (|C(t)|) .
$$

For the estimation of return densities we will need the semigroup of the Cauchy process while for risk neutral pricing we need to determine option prices.

Let us now derive the semigroup for absolute value of Cauchy. Let the candidate Lamperti process be

$$
L(u)=|C(u)|
$$

where $C(u)$ is the Cauchy process. Suppose that $L(u)=l$, and let $t>u$, we are interested in the density for $L(t)=y$ given that $L(u)=l$. By the homogeneous Markov property for the process $L$ we know that this density is of the form $q_{t-u}(l, y)$. Noting that $C(t)-C(u)$ is a Cauchy process started at zero, and taking $C(u)=c$, $t-u=h$, then for a test function $f(y)$, we may write 


$$
\begin{aligned}
E[f(L(t)) \mid C(u)=c] & =\frac{1}{\pi} \int_{-\infty}^{\infty} \frac{h}{h^{2}+x^{2}} f(|c+x|) d x \\
& =\frac{1}{\pi} \int_{-\infty}^{\infty} \frac{h}{h^{2}+(w-c)^{2}} f(|w|) d w \\
& =\frac{1}{\pi} \int_{0}^{\infty} \frac{h}{h^{2}+(w-c)^{2}} f(w) d w+\frac{1}{\pi} \int_{0}^{\infty} \frac{h}{h^{2}+(w+c)^{2}} f(w) d w \\
& =\frac{1}{\pi} \int_{0}^{\infty} \frac{2 h\left(h^{2}+w^{2}+c^{2}\right)}{\left(h^{2}+w^{2}+c^{2}\right)^{2}-4 w^{2} c^{2}} f(w) d w .
\end{aligned}
$$

It follows that the semigroup of $L(u)$ when starting at $l$ has density

$$
q_{h}(l, y)=\frac{2 h}{\pi} \frac{h^{2}+y^{2}+l^{2}}{\left(h^{2}+y^{2}+l^{2}\right)^{2}-4 y^{2} l^{2}} .
$$

For the normalization factor over $h$ units of time, noting that we start the Cauchy process at unity, we obtain from (4.17) that

$$
\begin{aligned}
e^{-\omega(h)} & =\int_{0}^{\infty} y^{-\beta} q_{h}(1, y) d y \\
& =\frac{2 h}{\pi} \int_{0}^{\infty} y^{-\beta} \frac{1+h^{2}+y^{2}}{\left(1+y^{2}+h^{2}\right)^{2}-4 y^{2}} d y
\end{aligned}
$$

The density for the stock price at time $t$ follows by the change of variable and we have the risk neutral density

$$
p_{t}(S)=q_{h}\left(1,\left(\frac{S}{S(0) e^{(r-q) t+\omega(t)}}\right)^{-\frac{1}{\beta}}\right)\left[\frac{1}{\beta S}\left(\frac{S}{S(0) e^{(r-q) t+\omega(t)}}\right)^{-\frac{1}{\beta}}\right]
$$

For a model that is to be useful in calibration we need to introduce parameters that allow for flexibility. In this regard we may introduce a volatility parameter by 
considering a time change whereby we let $h \rightarrow \sigma^{2} h$ and we write

$$
q_{h}(l, y)=\frac{2 \sigma^{2} h}{\pi} \frac{\sigma^{4} h^{2}+y^{2}+l^{2}}{\left(\sigma^{4} h^{2}+y^{2}+l^{2}\right)^{2}-4 y^{2} l^{2}} .
$$

One may also introduce a scale parameter so that the variable of interest is $c^{-1} L(t)$. This leads to

$$
\begin{aligned}
q_{h}(l, y) & =\frac{2 c \sigma^{2} h}{\pi} \frac{\sigma^{4} h^{2}+c^{2} y^{2}+c^{2} l^{2}}{\left(\sigma^{4} h^{2}+c^{2} y^{2}+c^{2} l^{2}\right)^{2}-4 c^{4} y^{2} l^{2}} \\
& =\frac{2 \sigma^{2} h}{\pi c} \frac{\frac{\sigma^{4}}{c^{2}} h^{2}+y^{2}+l^{2}}{\left(\frac{\sigma^{4}}{c^{2}} h^{2}+y^{2}+l^{2}\right)^{2}-4 y^{2} l^{2}} .
\end{aligned}
$$

This transformation is equivalent to the time change $h \rightarrow \sigma^{2} c^{-1} h$ and hence the scale transformation is absorbed by the time transformation. We therefor have a two parameter density for the stock price, with parameters $\sigma, \beta$. We have a volatility parameter and a shape parameter but as yet no skew parameter. The risk neutral density for the stock may be written with positive parameters $\sigma, \gamma$ with $\beta=1+\gamma$ as

$$
\begin{aligned}
p_{t}(S ; \sigma, \gamma) & =q_{\sigma^{2} h}\left(1,\left(\frac{S}{S(0) e^{(r-q) t+\omega(t)}}\right)^{-\frac{1}{1+\gamma}}\right)\left[\frac{1}{(1+\gamma) S}\left(\frac{S}{S(0) e^{(r-q) t+\omega(t)}}\right)^{-\frac{1}{1+\gamma}}\right] \\
e^{-\omega(h)} & =\frac{2 h}{\pi} \int_{0}^{\infty} y^{-(1+\gamma)} \frac{1+h^{2}+y^{2}}{\left(1+y^{2}+h^{2}\right)^{2}-4 y^{2}} d y .
\end{aligned}
$$

Finally, we come to the Lévy process when absolute value of Cauchy is the Lamperti process. It is shown in [71] proposition 2.3 that the L'evy process $\xi(t)$ yielding the absolute value of the Cauchy process as the Lamperti process has characteristic function 


$$
E[\exp (i u \xi(t))]=\exp \left(-t u \tanh \left(\frac{\pi u}{2}\right)\right)
$$

with Lévy measure

$$
k_{\xi}(x)=\frac{1}{\pi} \frac{\cosh (x)}{\sinh (x)^{2}} .
$$

Furthermore, we may see the process $\xi(t)$ as

$$
(\xi(t) ; t \geq 0) \stackrel{d}{=}\left(\int_{0}^{\tau_{t}} I_{\left\{\left|B_{s}\right| \leq \frac{\pi}{2}\right\}} d W_{s} ; t \geq 0\right)
$$

where $B$ and $W$ are independent Brownian motions and $\tau_{t}$ is the inverse of the local time at 0 of $B$.

We could attempt to build in skew by exponentially tilting the Lévy measure of this Lévy process. This is shown in the following section.

\subsubsection{Exponential Tilting of a Lévy Process in General}

Suppose we have a Lévy process $\xi(t)$ with Lévy measure $k(x)$ and characteristic exponent $\psi(u)$ defined as

$$
E[\exp (i u \xi(t))]=\exp (t \psi(u))
$$

Define the martingale

$$
M(t)=\exp (a \xi(t)-t \psi(-i a)))
$$

and let the law of $\xi^{(a)}$ be defined by

$$
E\left[F\left(\xi^{(a)}(u), u>0\right)\right]=E[F(\xi(u), u>0) M(T)]
$$


The characteristic function of $\xi^{(a)}$ follows from

$$
\begin{aligned}
E\left[e^{i u \xi^{(a)}(t)}\right] & =E\left[e^{i u \xi(t)} e^{a \xi(t)-t \psi(-i a)}\right] \\
& =\exp (t \psi(u-i a)-t \psi(-i a)) \\
& =\exp (t[\psi(u-i a)-\psi(-i a)]) .
\end{aligned}
$$

Now suppose

$$
\psi(u)=i u m-\frac{c^{2} u^{2}}{2}+\int_{-\infty}^{+\infty}\left(e^{i u x}-1-i u x I_{|x| \leq 1}\right) k(x) d x
$$

We then have

$$
\begin{gathered}
\psi(u-i a)=i(u-i a) m-\frac{c^{2}(u-i a)^{2}}{2}+\int_{-\infty}^{+\infty}\left(e^{i(u-i a) x}-1-i(u-i a) x I_{|x| \leq 1}\right) k(x) d x, \\
\psi(-i a)=a m+\frac{c^{2} a^{2}}{2}+\int_{-\infty}^{+\infty}\left(e^{a x}-1-a x I_{|x| \leq 1}\right) k(x) d x .
\end{gathered}
$$

We thereby obtain

$$
\begin{aligned}
\psi(u-i a)-\psi(-i a)= & i u\left(m+a c^{2}\right)-\frac{c^{2} u^{2}}{2}+\int_{-\infty}^{+\infty}\left(e^{i u x} e^{a x}-e^{a x}-i u x I_{|x| \leq 1}\right) k(x) d x \\
= & i u\left(m+a c^{2}\right)-\frac{c^{2} u^{2}}{2}+\int_{-\infty}^{+\infty}\left(e^{i u x}-1-i u x I_{|x| \leq 1}\right) e^{a x} k(x) d x \\
& +i u \int_{-1}^{1}\left(e^{a x}-1\right) x k(x) d x \\
= & i u\left(m+a c^{2}+\int_{-1}^{1}\left(e^{a x}-1\right) x k(x) d x\right)-\frac{c^{2} u^{2}}{2} \\
& +\int_{-\infty}^{+\infty}\left(e^{i u x}-1-i u x I_{|x| \leq 1}\right) e^{a x} k(x) d x .
\end{aligned}
$$

We see that the process $\xi^{(a)}(t)$ has the same diffusion coefficient, but an altered drift coefficient given by 


$$
m+a c^{2}+\int_{-1}^{1}\left(e^{a x}-1\right) x k(x) d x
$$

and exponentially tilted Lévy measure

$$
e^{a x} k(x) d x
$$

Skew may then be introduced by working with $\xi^{(a)}(t)$ in place of $\xi(t)$. 


\section{Chapter 5}

\section{THE DIFFUSION CASE WITH SQUARED BESSEL}

In this chapter, we construct a leveraged diffusion model. Void of jumps, the Brownian component is the only uncertainty that drives the model. A formulation similar to the one used in Chapter 4 is implemented. A natural choice of Bessel Squared process as the "Lamperti" is made since the family of Bessel Squared processes is the only family of continuous semi-stable Markov processes. We also estimate the model parameters both statistically and risk-neutrally.

\subsection{The Formulation}

We wish to maintain the growth rate of the stock and allow for an additional parameter in the volatility structure relative to what we were considering in the general formulation of Chapter 4. The growth rate for the stock is set to be $\mu(r-q$

in the risk-neutral world), and we suggest that the local absolute volatility be of the form

$$
\sigma S_{t}^{1+\alpha} e^{-\alpha \mu t},
$$

whereby the relative volatility is of the form

$$
\sigma\left(S_{t} e^{-\mu t}\right)^{\alpha},
$$

so that we have with $\alpha=0$, a constant volatility, while for $\alpha \neq 0$ we do not engineer any trend into the volatility, given the stock price is assumed to be trending at rate 
$\mu$. To expect the leverage effect, we would like to have $\alpha<0$. Noticing the change of parameter notation here, and in fact $\alpha+1$ is equivalent to the parameter $a$ in earlier general model (Section 4.2).

We would like $S_{t} e^{-\mu t}$ to be a positive martingale and hence take as a generic model the stochastic exponential of a martingale. $\exp \left(W_{t}-\frac{1}{2} t\right)$ is such Martingale in this case. We also incorporate the change in volatility using time changes and begin with the formulation

$$
S_{t} e^{-\mu t}=\exp \left(W\left(\int_{0}^{t} \sigma^{2} S_{u}^{2 \alpha} e^{-2 \mu \alpha u} d u\right)-\frac{1}{2} \int_{0}^{t} \sigma^{2} S_{u}^{2 \alpha} e^{-2 \mu \alpha u} d u\right)
$$

We now relate to Lamperti process by defining the time change

$$
\zeta(t)=\int_{0}^{t} \sigma^{2} S_{u}^{2 \alpha} e^{-2 \mu \alpha u} d u
$$

and let

$$
t=\int_{0}^{\eta(t)} \sigma^{2} S_{u}^{2 \alpha} e^{-2 \mu \alpha u} d u
$$

We note that

$$
\eta^{\prime}(t)=\frac{1}{\sigma^{2}} S_{\eta(t)}^{-2 \alpha} e^{2 \mu \alpha \eta(t)}
$$

Evaluating expression (5.1) at $\eta(t)$ we get

$$
S_{\eta(t)} e^{-\mu \eta(t)}=\exp \left(W(t)-\frac{t}{2}\right)
$$

It follows that 


$$
S_{\eta(t)}^{-2 \alpha}=\exp \left(-2 \alpha W_{t}+\alpha t\right) e^{-2 \alpha \mu \eta(t)}
$$

SO

$$
\eta^{\prime}(t)=\frac{1}{\sigma^{2}} \exp \left(-2 \alpha W_{t}+\alpha t\right)
$$

Integrating we get that

$$
\eta(t)=\frac{1}{\sigma^{2}} \int_{0}^{t} e^{-2 \alpha W_{s}+\alpha s} d s .
$$

Let

$$
\xi^{(\alpha)}(t)=-2 \alpha W_{t}+\alpha t
$$

we have from Lamperti's relation

$$
\begin{aligned}
\exp \left(-2 \alpha W_{t}+\alpha t\right) & =L^{\left(\xi^{(\alpha)}\right)}\left(\int_{0}^{t} e^{-2 \alpha W_{s}+\alpha s} d s\right) \\
& =L^{\left(\xi^{(\alpha)}\right)}\left(\sigma^{2} \eta(t)\right)
\end{aligned}
$$

But we also have

$$
S_{\eta(t)}^{-2 \alpha} e^{2 \alpha \mu \eta(t)}=\exp \left(-2 \alpha W_{t}+\alpha t\right)
$$

together we obtain the relation

$$
S_{\eta(t)}^{-2 \alpha} e^{2 \alpha \mu \eta(t)}=L^{\left(\xi^{(\alpha)}\right)}\left(\sigma^{2} \eta(t)\right)
$$

Using the scaling property of Lamperti processes, the general model for the stock price in diffusion case is 


$$
S_{t}=S_{0}\left\{e^{-2 \alpha \mu t} \sigma^{2} L^{\left(\xi^{(\alpha)}\right)}(t)\right\}^{-\frac{1}{2 \alpha}}
$$

It is a Lamperti process raised to a power.

\subsection{Take Bessel Squared Process as the Lamperti}

Bessel Squared Process of dimension $\delta\left(\mathrm{BESQ}^{\delta}\right) Z_{t}^{(\delta)}$ has SDE

$$
d Z_{t}^{(\delta)}=\delta d t+2 \sqrt{Z_{t}^{(\delta)}} d W_{t}
$$

Since BESQ is the only family of continuous Lamperti processes, we replace the Lamperti process in (5.2) with $Z_{t}^{(\delta)}$, where by Martingale property

$$
\delta=\frac{1}{\alpha}+2
$$

We have for stock price model:

$$
S_{t}=S_{0} e^{\mu t}\left\{\sigma^{2} Z_{t}^{(\delta)}\right\}^{\frac{2-\delta}{2}} .
$$

where $Z_{t}^{(\delta)}$ is BESQ process of dimension $\delta$. Knowing the SDE for $Z_{t}^{(\delta)}$ and (5.3), the SDE of $S_{t}$ is easily derived by applying Ito's Lemma

$$
d S_{t}=\mu S_{t} d t+\sigma(2-\delta)\left(\frac{S_{t}}{S_{0} e^{\mu t}}\right)^{\frac{1}{\delta-2}} S_{t} d W_{t} .
$$

In the risk-neutral setting, $\mu=r$ the interest rate. Use $l$ (to avoid confusion with $q$ as density function in the following sections) to denote the dividend yield, our generalized SDE in the risk-neutral world is

$$
d S_{t}=(r-l) S_{t} d t+\sigma(2-\delta)\left(\frac{S_{t}}{S_{0} e^{(r-l) t}}\right)^{\frac{1}{\delta-2}} S_{t} d W_{t} .
$$




\subsection{Maximum Likelihood Estimation of Parameters}

We want to estimate the parameters $\mu, \sigma(\sigma>0)$ and $\delta$. Parameter $\delta$ is the dimension parameter (we only consider positive dimension here) from the BESQ process and it is related to the power parameter $\alpha$ by $\delta=\frac{1}{\alpha}+2$. For the leverage effect, we expect $0<\delta<2$.

The transition density of BESQ process $Z_{t}^{(\delta)}$ is:

$$
q_{h}^{\delta}(x, y)=\frac{1}{2 h}\left(\frac{y}{x}\right)^{\nu / 2} \exp \left(-\frac{x+y}{2 h}\right) I_{\nu}\left(\frac{\sqrt{x y}}{h}\right)
$$

where $\nu=\frac{\delta}{2}-1$ and $I_{\nu}$ is the modified Bessel function of the first kind with index $\nu$. According to the relation in (5.3), and with a simple variable change, the transition density of $S_{t}$ is given by:

$$
q_{h}^{S_{t}}\left(S_{t}, S_{t+h}\right)=q_{h}^{\delta}\left(Z_{t}, Z_{t+h}\right)\left|\frac{\partial Z_{t+h}}{\partial S_{t+h}}\right|
$$

Assuming the time series of stock prices $S_{t_{0}}, S_{t_{1}}, S_{t_{2}}, \ldots, S_{t_{n}}$, corresponding to time $t_{0}=0, t_{1}, t_{2}, \ldots, t_{n}$, and change of time $\Delta_{i}:=t_{i+1}-t_{i}(i=1,2, \ldots, n-1)$, and the log likelihood function is defined as

$$
L(\mu, \sigma, \delta)=\sum_{i=1}^{n-1} \ln q_{\Delta_{i}}^{S_{t}}\left(S_{t_{i}}, S_{t_{i+1}}\right)
$$

We want to maximize $L$ with respect to $\mu, \sigma$ and $\delta$ according the sample.

\subsubsection{Data and Results}

We implement the Maximum Likelihood Estimation using the optimization tool in Matlab. Before going into the MLE for real data, we first test our optimiza- 
tion routine on simulated stock prices. Since we know the transition density of $S_{t}$ is given explicitly by (5.6), at each time point $t$, knowing the value of $S$ at time $t, x$, we could generate the conditional cdf of $y$, the value of $S$ at $t+h$, by specifying the range of movement of $S$ from $t$ to $t+h$. Specifically, we pre-specify $T$, number of years, $h$ the step size in time, hence $M=T / h$ the number of observations. The initial value $S_{0}$ is set to be 0.5 and the parameters $\mu, \sigma$ and $\delta$ are set to be $0.32,0.6$ and 1.5 respectively. At each time $t=[0, h, 2 h, \ldots, T]$, knowing value $S_{t}, x$ and the specified possible values of $S_{t+h}$, for example $y=[x-$ movement $:$ stepsize $: x+$ movement $]$ for some chosen "movement" and "step size", we use (5.6) to calculate $q$ values. After normalizing the $q^{\prime} s$, the cdf of $y$ given $x$ is simply obtained by command "cumsum" in Matlab. In the end, we use the Inverse Transform simulation technique to obtain a random value $y$ at time $t+h$ with the help of cubic spline interpolation and a randomly generated number. The simulated data will then be put into the test of Maximum Likelihood Estimation to see if the estimated values for parameters $\mu$, $\sigma$ and $\delta$ are indeed the true values $0.32,0.6$ and 1.5 . We selectively show some results of MLE on simulated data in Table 5.1. $N$ is the number of replications of simulation.

We observe from Table 5.1 that as we increase the number of observations and number of replications of simulation, the estimated parameters are convergent to the true values. The optimization routine is ready to be used on market data to provide trustable results.

Next, the MLE is implemented on $S \& P 500$ Index daily data from September 21, 1983 to September 20, 2004. In these 21 years, there are 5298 observations 
Table 5.1: Maximum Likelihood Estimation Results on Simulated Data. True Value: $\mu=0.32, \sigma=0.6, \delta=1.5$

\begin{tabular}{|c|c|c|}
\hline$h=0.01$ & $T=1$ & $N=100$ \\
\hline$\hat{\mu}$ & 0.2799 & $0.1231(s t d)$ \\
$\hat{\sigma}$ & 0.6566 & $0.3007(s t d)$ \\
$\hat{\delta}$ & 1.3982 & $0.7613(s t d)$ \\
\hline$h=0.01$ & $T=2$ & $N=100$ \\
\hline$\hat{\mu}$ & 0.3108 & $0.0540(s t d)$ \\
$\hat{\sigma}$ & 0.5886 & $0.1398(s t d)$ \\
$\hat{\delta}$ & 1.4862 & $0.1185(s t d)$ \\
\hline$h=0.01$ & $T=4$ & $N=200$ \\
\hline$\hat{\mu}$ & 0.3169 & $0.0161(s t d)$ \\
$\hat{\sigma}$ & 0.5913 & $0.0587(s t d)$ \\
$\hat{\delta}$ & 1.4954 & $0.0503(s t d)$ \\
\hline
\end{tabular}

excluding the initial $S_{0}=168.41$. A plot of the data is shown in Figure 5.1. The estimated $\mu, \sigma$ and $\delta$ are respectively:

$$
\hat{\mu}=0.1389 ; \quad \hat{\sigma}=0.0777 ; \quad \hat{\delta}=0.0024 \text {. }
$$

which suggests the model of price as

$$
d S_{t} / S_{t}=0.14 d t+2 e^{0.07 t} S_{t}^{-0.5} d W_{t}
$$

Here $\hat{\delta}$ is between 0 and 2 which implies a "Leverage" effect in this model. 
Figure 5.1: $S \& P 500$ Daily Prices from 09/21/1983 to 09/20/2004

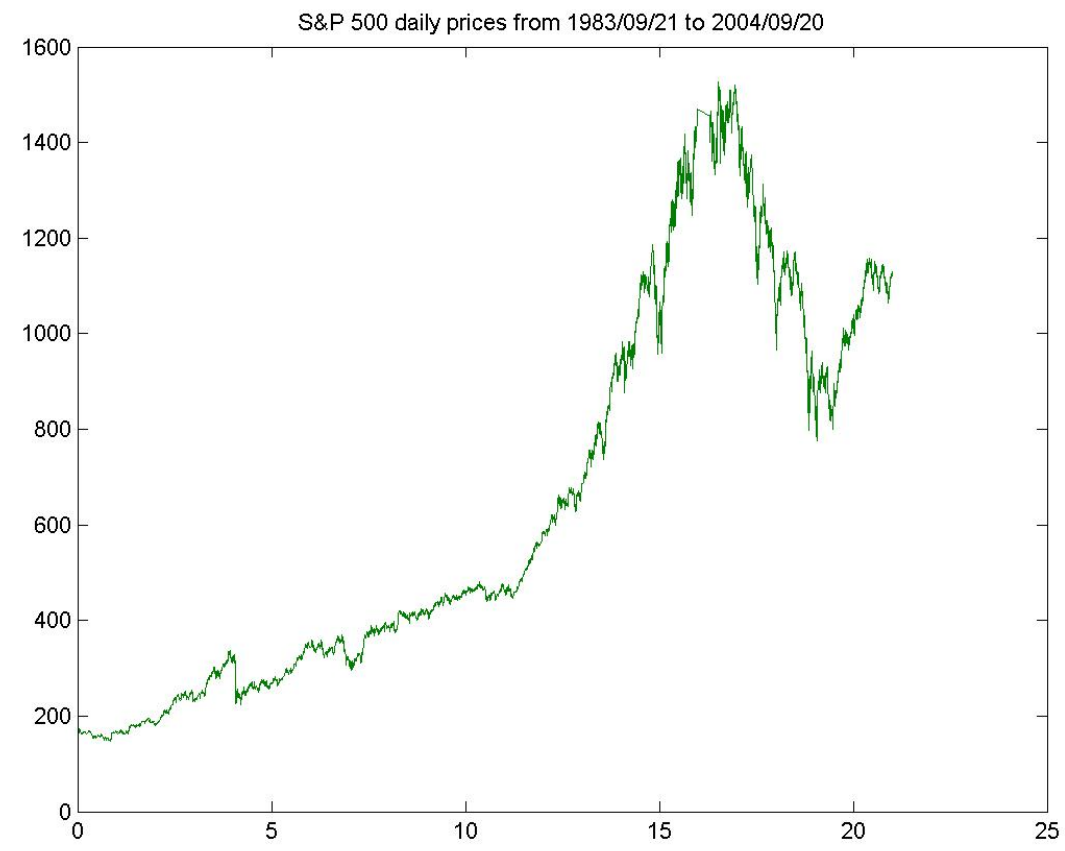

Now, we implement the same Maximum Likelihood Estimation on individual stocks and hope to see leverage effect prevails as well. All data is drawn from CRSP daily stock prices, available for a 10-year period from December 1, 1993 to December 31, 2003. We order the $S \& P 500$ component companies by the estimated Market Capitalization percentage. The top 60 companies are chosen to be examined. The estimated Market Capitalization is calculated by multiplying the number of shares outstanding times previous close price of the last day of this 10-year period. This number reflects the total dollar value of a company's outstanding shares.

We also implement a hypothesis test of our leveraged Lévy model (5.4) against the Geometric Brownian Model. In order for the GBM case to nest in our model here, we define 


$$
V_{t}=S_{t} e^{-\mu t}
$$

and derive our hypothesis:

$H_{0}$ : the GBM model $\left(d S_{t}=\mu S_{t} d t+\sigma S_{t} d W_{t}\right)$

$$
d V_{t}=\sigma V_{t} d W_{t}
$$

$H_{1}$ : leveraged Lévy model $\left(d S_{t}=\mu S_{t} d t+\sigma(2-\delta)\left(\frac{S_{t}}{S_{0} e^{\mu t}}\right)^{\frac{1}{\delta-2}} S_{t} d W_{t}\right)$

$$
d V_{t}=\sigma(2-\delta)\left(\frac{V_{t}}{V_{0}}\right)^{\frac{1}{\delta-2}} V_{t} d W_{t}
$$

Let $\frac{\Delta V_{t}}{V_{t}}=R_{t}$, so we see that in $H_{0}, R_{t}$ is Normally distributed as $N\left(0, \sigma^{2} \Delta_{i}\right)$ and in $H_{1}, R_{t}$ is Normally distributed as $N\left(0, \gamma \Delta_{i}\right)$, where

$$
\gamma=\sigma^{2}(2-\delta)^{2}\left(\frac{V_{t}}{V_{0}}\right)^{\frac{2}{\delta-2}}
$$

Let

$$
L_{0}=-\ln \left(\prod_{i=1}^{n-1} \frac{e^{-R_{t}^{2}(i) / 2 \sigma^{2} \Delta_{i}}}{\sqrt{2 \pi \sigma^{2} \Delta_{i}}}\right)=-\sum_{i=1}^{n-1} R_{t}^{2}(i) / 2 \sigma^{2} \Delta_{i}-(n-1) \ln \sqrt{2 \pi \sigma^{2} \Delta_{i}}
$$

where $R_{t}(i)=\frac{V(i+1)-V(i)}{V(i)}, i=1, \ldots, n-1$, and

$$
L_{1}=-\ln \left(\prod_{i=1}^{n-1} \frac{e^{-R_{t}^{2}(i) / 2 \gamma \Delta_{i}}}{\sqrt{2 \pi \gamma \Delta_{i}}}\right)=-\sum_{i=1}^{n-1} R_{t}^{2}(i) / 2 \gamma \Delta_{i}-(n-1) \ln \sqrt{2 \pi \gamma \Delta_{i}}
$$

We set the significance level at $0.05=5 \%$. Since the degree of freedom is 1 , our critical value for the chi-squared distribution is 3.84 . Compare the ratio $-2 \frac{L_{0}}{L_{1}}$ 
with this critical value, a ratio larger than 3.84 leads to rejecting hypothesis $H_{0}$ and is favorable to us. The results are shown in Table 5.2, Table 5.3 and Table 5.4.

In these two tables, the leverage effect can be observed when $\frac{1}{\delta-2}$ is negative. Only 7 out of 60 tested cases have this effect. We see that the Geometric Brownian Model is 100 percent rejected which supports our "leveraged" model being better. However, the relation between volatility and the spot price is seemingly more "positive" than "negative" in these top 60 companies. Although the observation here deviates from our expectation, it confirms the existence of leverage. The results are in fact consistent with another rising argument, which argues that volatilities rise when stock prices go up. This is because they reach bubble territory or levels unsupported by earnings and thus there is increasing uncertainty about their ability to maintain such levels.

Being curious, we explore the same tests on another 19 companies, but this time the companies are chosen to be the ones of lowest credit rating. The Reuters credit report for $S \& P 500$ companies is used. Interestingly, 8 out of the 19 cases have parameters indicating leverage. This is a much higher percentage than the leverage result observed from the top 60 companies, which is only 7 out of 60 . Table 5.5 shows a summary of MLE result for these 19 companies. This result confirms our leverage consideration and is consistent with the argument of reaching bubble territory. For these companies that are not "doing so well", they are further away from reaching the bubble territory so that the market fear of price drop is more of the driving effect to the movement of the volatility. 
Table 5.2: Summary of MLE Result of Top 60 Companies in $S \& P 500$, Part One

\begin{tabular}{|c|c|c|c|c|c|c|c|c|}
\hline Ticker & Data & $\hat{\mu}$ & $\hat{\sigma}$ & $\hat{\delta}$ & $\frac{1}{\delta-2}$ & Leveraged? & $-2 \frac{L_{0}}{L_{1}}$ & $H_{1} ?$ \\
\hline MSFT & 2540 & 0.0257 & 0.05 & 8.2759 & 0.1593 & No & 134.8534 & Yes \\
INTC & 2540 & -0.5175 & 0.0322 & 13.0514 & 0.0905 & No & 260.3315 & Yes \\
CSCO & 2540 & -0.0243 & 0.0424 & 13.7732 & 0.0849 & No & 89.4885 & Yes \\
GE & 2540 & -0.2626 & 0.034 & 8.0783 & 0.1645 & No & 288.5841 & Yes \\
ABC & 2203 & 0.2177 & 0.3687 & 0.7076 & -0.7737 & Yes & 309.3126 & Yes \\
PFE & 2540 & 0.0832 & 0.0604 & 6.5447 & 0.2200 & No & 185.9992 & Yes \\
DELL & 2540 & 0.7661 & 0.054 & 13.9961 & 0.0834 & No & 82.7444 & Yes \\
C & 2540 & 0.0434 & 0.0476 & 9.372 & 0.1356 & No & 69.7444 & Yes \\
XOM & 2540 & 0.0131 & 0.0926 & 3.8939 & 0.5280 & No & 238.2123 & Yes \\
AMAT & 2540 & -0.0944 & 0.0448 & 15.2958 & 0.0752 & No & 105.9012 & Yes \\
IBM & 2540 & 0.0291 & 0.0462 & 9.0602 & 0.1416 & No & 116.9723 & Yes \\
YHOO & 1943 & 0.6803 & 0.0501 & 20 & 0.0556 & No & 46.3246 & Yes \\
MRK & 2540 & -0.0539 & 0.0414 & 8.0521 & 0.1652 & No & 125.986 & Yes \\
CAH & 2540 & 0.2697 & 0.199 & 0.3627 & -0.6107 & Yes & 132.5642 & Yes \\
WMT & 2540 & 0.0772 & 0.0449 & 9.8492 & 0.1274 & No & 125.4873 & Yes \\
BMGN & 2540 & 0.062 & 0.063 & 8.0882 & 0.1643 & No & 162.254 & Yes \\
ORCL & 2540 & -0.2135 & 0.0402 & 15.3509 & 0.0749 & No & 153.6999 & Yes \\
\hline
\end{tabular}


Table 5.3: Summary of MLE Result of Top 60 Companies in S\&P500, Part Two

\begin{tabular}{|c|c|c|c|c|c|c|c|c|}
\hline BRCM & 1435 & 0.3471 & 0.0554 & 20.0108 & 0.0555 & No & 42.1781 & Yes \\
JNJ & 2540 & 0.003 & 0.0378 & 8.3314 & 0.1579 & No & 95.4829 & Yes \\
GS & 1172 & 0.719 & 0.2041 & 5.4658 & 0.2885 & No & 187.0127 & Yes \\
KLAC & 2540 & -0.0552 & 0.0434 & 18.0188 & 0.0624 & No & 66.0579 & Yes \\
TWX & 2540 & 0.656 & 0.053 & 15.0244 & 0.0768 & No & 72.693 & Yes \\
QCOM & 2540 & 0.3045 & 0.0501 & 16.5568 & 0.0687 & No & 134.5756 & Yes \\
AIG & 2540 & -0.0909 & 0.0529 & 6.0518 & 0.2468 & No & 253.3821 & Yes \\
LLY & 2540 & -0.0419 & 0.0459 & 8.4642 & 0.1547 & No & 173.0266 & Yes \\
MO & 2540 & 0.2068 & 0.1679 & $2.48 \mathrm{E}-04$ & -0.5001 & Yes & 121.876 & Yes \\
CVX & 2540 & 0.0531 & 0.1331 & 3.5587 & 0.6416 & No & 156.3164 & Yes \\
FNM & 2540 & 0.0973 & 0.0424 & 9.4846 & 0.1336 & No & 78.1516 & Yes \\
NXTL & 2540 & -0.9658 & 0.0387 & 17.4686 & 0.0646 & No & 179.1065 & Yes \\
VZ & 2540 & -0.3458 & 0.0357 & 8.5309 & 0.1531 & No & 231.2349 & Yes \\
JPM & 2540 & -0.4458 & 0.0341 & 9.8419 & 0.1275 & No & 239.4546 & Yes \\
PG & 2540 & -0.0559 & 0.0562 & 6.5346 & 0.2205 & No & 160.0144 & Yes \\
MCK & 2287 & -0.111 & 0.1185 & 4.0868 & 0.4792 & No & 276.7385 & Yes \\
NEM & 2540 & -0.0523 & 0.0484 & 8.9672 & 0.1435 & No & 105.1398 & Yes \\
\hline
\end{tabular}


Table 5.4: Summary of MLE Result of Top 60 Companies in S\&P500, Part Three

\begin{tabular}{|c|c|c|c|c|c|c|c|c|}
\hline WFC & 2540 & 0.2054 & 0.0523 & 8.0183 & 0.1662 & No & 94.241 & Yes \\
CAT & 2540 & -0.039 & 0.0421 & 9.9601 & 0.1256 & No & 60.841 & Yes \\
TXN & 2540 & -0.4109 & 0.0361 & 14.6575 & 0.0790 & No & 203.8517 & Yes \\
HD & 2540 & -0.3925 & 0.0349 & 10.3468 & 0.1198 & No & 271.1829 & Yes \\
KO & 2540 & -0.1586 & 0.0386 & 7.7848 & 0.1729 & No & 146.9029 & Yes \\
MXIM & 2540 & -0.1797 & 0.0408 & 15.8084 & 0.0724 & No & 133.2019 & Yes \\
PEP & 2540 & 0.0254 & 0.0415 & 9.4295 & 0.1346 & No & 85.2386 & Yes \\
SBC & 2540 & -0.3796 & 0.0341 & 9.1148 & 0.1406 & No & 260.6096 & Yes \\
ERTS & 2540 & 0.5452 & 0.0577 & 15.6207 & 0.0734 & No & 75.5943 & Yes \\
MWD & 2540 & -0.3702 & 0.0353 & 12.0341 & 0.0997 & No & 225.9111 & Yes \\
CIEN & 1734 & 0.751 & 0.0449 & 29.936 & 0.0358 & No & 69.228 & Yes \\
ABT & 2540 & -0.0939 & 0.042 & 8.3587 & 0.1573 & No & 130.4067 & Yes \\
TYC & 2540 & 0.5017 & 0.1135 & 0.0092 & -0.5023 & Yes & $1.06 E+03$ & Yes \\
MER & 2540 & -0.0302 & 0.0581 & 8.6304 & 0.1508 & No & 131.5425 & Yes \\
FDC & 2540 & 0.1777 & 0.3782 & 1.1962 & -1.2441 & Yes & 445.1442 & Yes \\
COP & 2540 & 0.1073 & 0.2022 & 0.449 & -0.6447 & Yes & 66.5387 & Yes \\
WYE & 2540 & -0.2977 & 0.0515 & 5.6907 & 0.2710 & No & 488.7357 & Yes \\
HUD & 2540 & -0.0698 & 0.0395 & 7.5176 & 0.1812 & No & 180.543 & Yes \\
\hline
\end{tabular}


Table 5.5: Summary of MLE Result of Low 19 Companies in S\&P500

\begin{tabular}{|c|c|c|c|c|c|c|c|c|}
\hline Ticker & Data & $\hat{\mu}$ & $\hat{\sigma}$ & $\hat{\delta}$ & $\frac{1}{\hat{\delta}-2}$ & Leveraged? & $-2 \frac{L_{0}}{L_{1}}$ & $H_{1} ?$ \\
\hline GLK & 2540 & -0.0415 & 0.2211 & 0.8984 & -0.9078 & Yes & 179.7616 & Yes \\
RBK & 2540 & 0.0349 & 0.2359 & 0.4761 & -0.6562 & Yes & 665.1394 & Yes \\
HPC & 2540 & 0.0162 & 0.1565 & $4.02 \mathrm{E}-04$ & -0.5001 & Yes & 451.7621 & Yes \\
GTW & 2535 & -0.4139 & 0.0398 & 17.6878 & 0.0637 & No & 88.5499 & Yes \\
PLL & 2540 & 0.0482 & 0.1917 & 0.0869 & -0.5227 & Yes & 98.9453 & Yes \\
PKI & 2540 & -0.6418 & 0.0395 & 9.2756 & 0.1374 & No & 472.6433 & Yes \\
AW & 2540 & 0.1289 & 0.0452 & 16.3807 & 0.0695 & No & 36.1774 & Yes \\
FSH & 2540 & -0.1693 & 0.0401 & 11.7491 & 0.1026 & No & 118.9657 & Yes \\
TMK & 2540 & -0.0944 & 0.0423 & 8.061 & 0.16499 & No & 75.1405 & Yes \\
PWER & 1571 & -0.3288 & 0.0518 & 20.1252 & 0.0552 & No & 69.1856 & Yes \\
MIL & 2540 & -0.325 & 0.037 & 10.1312 & 0.1230 & No & 179.7226 & Yes \\
MEP & 2540 & 0.1373 & 0.0492 & 7.9746 & 0.16734 & No & 63.6244 & Yes \\
R R & 2540 & 0.0521 & 0.2953 & 1.0796 & -1.0865 & Yes & 328.2285 & Yes \\
BLI & 2540 & -0.2109 & 0.0468 & 12.0394 & 0.0996 & No & 64.481 & Yes \\
MYG & 2540 & -0.3555 & 0.0381 & 9.6224 & 0.1312 & No & 216.7474 & Yes \\
PMTC & 2540 & 0.2714 & 0.052 & 7.8122 & 0.1721 & No & 66.6923 & Yes \\
\hline MKC & 2540 & 0.1091 & 0.21 & 0.9159 & -0.9224 & Yes & 78.3922 & Yes \\
\hline
\end{tabular}




\subsection{Forward PDE in The Diffusion Case}

In Sections 6.4 and 6.5 we derive in the general framework the Backward Partial Integro-Differential Equations and Forward Partial Integro-Differential Equations for option pricing. In these sections, the processes are general Markov processes without any further assumptions. Here we use the result in Section 6.5 and develop the forward equations in our BESQ case.

We consider the log price process $X_{t}=\ln \left(S_{t}\right)$, and then apply Ito's lemma to (5.4) to obtain

$$
d X_{t}=\left[r-l-\frac{1}{2} \sigma^{2}(2-\delta)^{2}\left(\frac{e^{X_{t}}}{S_{0} e^{(r-l) t}}\right)^{\frac{2}{\delta-2}}\right] d t+\sigma(2-\delta)\left(\frac{e^{X_{t}}}{S_{0} e^{(r-l) t}}\right)^{\frac{1}{\delta-2}} d W_{t}
$$

We know that in this case, we have a diffusion model without the jump component.

The generator of $X_{t}$ is

$$
I_{X}(f)(x, u)=a(x, u) \frac{\partial f}{\partial x}+\frac{1}{2} b(x, u) \frac{\partial^{2} f}{\partial x^{2}}
$$

where

$$
\begin{gathered}
a(x, u)=r-l-\frac{1}{2} \sigma^{2}(2-\delta)^{2}\left(\frac{e^{X_{t}}}{S_{0} e^{(r-l) t}}\right)^{\frac{2}{\delta-2}} \\
b(x, u)=\sigma^{2}(2-\delta)^{2}\left(\frac{e^{x}}{S_{0} e^{(r-l) u}}\right)^{\frac{2}{\delta-2}} .
\end{gathered}
$$

Hence, by the result in $(6.26)$, we have 


$$
\begin{aligned}
q_{T}(y, T)= & -\frac{\partial}{\partial y}[a(y, T) q(y, T)]+\frac{1}{2} \frac{\partial^{2}}{\partial y^{2}}[b(y, T) q(y, T)] \\
= & -(r-l) \frac{\partial}{\partial y} q(y, T)+\frac{\sigma^{2}(2-\delta)^{2}}{2\left(S_{0} e^{(r-l) T}\right)^{\frac{2}{\delta-2}}} \frac{\partial}{\partial y}\left[e^{\frac{2 y}{\delta-2}} q(y, T)\right] \\
& +\frac{\sigma^{2}(2-\delta)^{2}}{2\left(S_{0} e^{(r-l) T}\right)^{\frac{2}{\delta-2}}} \frac{\partial^{2}}{\partial y^{2}}\left[e^{\frac{2 y}{\delta-2}} q(y, T)\right] .
\end{aligned}
$$

For European Call option, we know that

$$
C(K, T)=e^{-r T} \int_{\ln K}^{\infty} q(y, T)\left(e^{y}-K\right) d y .
$$

Again $q(t, x, T, y)$ denotes the transition density for the process $X(t)$ to be at level $y$ for time $T>t$, given it is at level $x$ at time $t$. We keep the dependence of $q$ on $y$ and $T$, and suppress the dependence on $x$ and $t$.

It is easy to see that

$$
\begin{array}{r}
C_{K}=-e^{-r T} \int_{\ln K}^{\infty} q(y, T) d y, \\
e^{-r T} \int_{\ln K}^{\infty} e^{y} q(y, T) d y=C-K C_{K}, \\
K C_{K K}(K, T)=e^{-r T} q(\ln K, T),
\end{array}
$$

and

$$
C_{T}=-r C+e^{-r T} \int_{\ln K}^{\infty} q_{T}(y, T)\left(e^{y}-K\right) d y .
$$


Putting $q_{T}(y, T)$ into (5.8), we have

$$
\begin{aligned}
C_{T}= & -r C-(r-l) e^{-r T} \int_{\ln K}^{\infty}\left(e^{y}-K\right) \frac{\partial}{\partial y} q(y, T) d y \\
& +\frac{e^{-r T} \sigma^{2}(2-\delta)^{2}}{2\left(S_{0} e^{(r-l) T}\right)^{\frac{2}{\delta-2}}} \int_{\ln K}^{\infty}\left(e^{y}-K\right) \frac{\partial}{\partial y}\left[e^{\frac{2 y}{\delta-2}} q(y, T)\right] d y \\
& +\frac{e^{-r T} \sigma^{2}(2-\delta)^{2}}{2\left(S_{0} e^{(r-l) T}\right)^{\frac{2}{\delta-2}}} \int_{\ln K}^{\infty}\left(e^{y}-K\right) \frac{\partial^{2}}{\partial y^{2}}\left[e^{\frac{2 y}{\delta-2}} q(y, T)\right] d y \\
= & -r C+(r-l) e^{-r T} \int_{\ln K}^{\infty} e^{y} q(y, T) d y \\
& +\frac{e^{-r T} \sigma^{2}(2-\delta)^{2}}{2\left(S_{0} e^{(r-l) T}\right)^{\frac{2}{\delta-2}}}\left[-\int_{\ln K}^{\infty} e^{\frac{\delta y}{\delta-2}} q(y, T) d y\right] \\
& +\frac{e^{-r T} \sigma^{2}(2-\delta)^{2}}{2\left(S_{0} e^{(r-l) T}\right)^{\frac{2}{\delta-2}}}\left[K K^{\frac{2}{\delta-2}} q(\ln K, T)+\int_{\ln K}^{\infty} e^{\frac{\delta y}{\delta-2}} q(y, T) d y\right] \\
= & -r C+(r-l)\left(C-K_{K}\right)+\frac{\sigma^{2}(2-\delta)^{2} K^{\frac{\delta}{\delta-2}}}{2\left(S_{0} e^{(r-l) T}\right)^{\frac{2}{\delta-2}}} K C_{K K}(K, T) \\
= & -l C-(r-l) K C_{K}+\frac{\sigma^{2}(2-\delta)^{2} K^{\frac{2 \delta-2}{\delta-2}}}{2\left(S_{0} e^{(r-l) T}\right)^{\frac{2}{\delta-2}}} C_{K K}(K, T)
\end{aligned}
$$

We switch to the log strike space by defining

$$
k=\ln K .
$$

This transform will benefit the discretization of the system. This is because strike prices collected from real market data are often sparsely distributed in a large range, which brings difficulty to the discretization of the system when we always require the spacing of $K$ be small enough to achieve convergence. Because of the large range of strike prices, small spacing means too many points which substantially slow down the calculation, although most of these points are not even traded strikes of our interest. By the transform from strike space to log strike space, we can achieve the small spacing without making the system huge. We define 


$$
c(k, T)=C\left(e^{k}, T\right)=C(K, T)
$$

so that

$$
K C_{K}(K, T)=c_{k}(k, T) \quad C_{K K}(K, T)=e^{-2 k}\left[c_{k k}(k, T)-c_{k}(k, T)\right] .
$$

then, the forward PDE becomes

$$
\begin{aligned}
c_{T}(k, T)= & -l c-(r-l) c_{k}(k, T) \\
& +\frac{\sigma^{2}(2-\delta)^{2} e^{\frac{2 k}{\delta-2}}}{2\left(S_{0} e^{(r-l) T}\right)^{\frac{2}{\delta-2}}}\left[c_{k k}(k, T)-c_{k}(k, T)\right]
\end{aligned}
$$

\subsubsection{Discretization}

We discretize this system with $N+1$ mesh points in $k$-direction and $M+1$ mesh points in $T$-direction. So:

$$
\Delta k=\frac{k_{\max }-k_{\min }}{N}, \quad \Delta T=\frac{T_{\max }-T_{\min }}{M} .
$$

Hence $T_{1}=T_{\min }, T_{M+1}=T_{\max }, T_{j}=T_{1}+(j-1) \Delta T$ for all $j=1, \ldots, M+1$, and $k_{1}=k_{\text {min }}, k_{N+1}=k_{\max }, k_{i}=k_{1}+(i-1) \Delta k$ for all $i=1, \ldots, N+1$, where $k_{\min }=\ln K_{\min }, k_{\max }=\ln K_{\max }$.

Using

$$
c_{i, j} \approx c\left(k_{i}, T_{j}\right)
$$

we approximate the derivatives in $k$ using centered differences:

$$
c_{T}\left(k_{i}, T_{j}\right) \approx \frac{c_{i, j+1}-c_{i, j}}{\Delta T}
$$




$$
\begin{gathered}
c_{k}\left(k_{i}, T_{j}\right) \approx \frac{c_{i+1, j}-c_{i-1, j}}{2 \Delta k} ; \\
c_{k k}\left(k_{i}, T_{j}\right) \approx \frac{c_{i+1, j}+c_{i-1, j}-2 c_{i, j}}{(\Delta k)^{2}} .
\end{gathered}
$$

Initial condition

$$
c\left(k_{i}, T_{1}\right)=\left[S_{T_{1}}-\exp \left(k_{i}\right)\right]^{+} \text {for all } i=1, \ldots, N+1 .
$$

Boundary conditions

$$
\begin{gathered}
c\left(k_{1}, T_{j}\right)=S_{T_{1}} e^{-l T_{j}}-\exp \left(k_{1}\right) e^{-r T_{j}} \text { for all } j=1, \ldots, M+1, \\
c\left(k_{N+1}, T_{j}\right)=0 \text { for all } j=1, \ldots, M+1 .
\end{gathered}
$$

Using these notations, now we discretize equation (5.9) using implicit scheme.

$$
\begin{array}{r}
\frac{c_{i, j+1}-c_{i, j}}{\Delta T}=-l c_{i, j+1}-(r-l) \frac{c_{i+1, j+1}-c_{i-1, j+1}}{2 \Delta K} \\
+\frac{\sigma^{2}(2-\delta)^{2} e^{\frac{2 k_{i}}{\delta-2}}}{2\left(S_{0} e^{(r-l) T_{j+1}}\right)^{\frac{2}{\delta-2}}}\left[\frac{c_{i+1, j+1}+c_{i-1, j+1}-2 c_{i, j+1}}{(\Delta k)^{2}}-\frac{c_{i+1, j+1}-c_{i-1, j+1}}{2 \Delta k}\right]
\end{array}
$$

we set $\lambda=\frac{\Delta T}{2 \Delta k}, \gamma=\frac{\sigma^{2}(2-\delta)^{2}}{2 S_{0} \frac{2}{\delta-2}}$, and rearrange (5.10) to find the following discretized system. Indeed, the interest rates and dividend yields are not constants as what we have presented in the previous formulation. In fact they vary across time. According to the data we obtain together with strikes and maturities, we calculate all the needed interest rates and dividend rates by assuming piece wise constant forward rates. In the following discretized system, we will see $r$ and $l$ both have time index. 


$$
\begin{aligned}
& {\left[(l-r) \lambda-\frac{\left.(2+\Delta k) \Delta T \gamma e^{\frac{2}{\delta-2}\left[k_{i}-\left(r_{j+1}-l_{j+1}\right) T_{j+1}\right]}\right] c_{i-1, j+1}}{2(\Delta k)^{2}}\right] c_{i, j+1} } \\
+ & {\left[1+l \Delta T+\frac{2 \Delta T \gamma e^{\frac{2}{\delta-2}\left[k_{i}-\left(r_{j+1}-l_{j+1}\right) T_{j+1}\right]}}{(\Delta k)^{2}}\right] c_{i+1, j+1} } \\
+ & {\left[\left(r_{j+1}-l_{j+1}\right) \lambda+\frac{(\Delta k-2) \Delta T \gamma e^{\frac{2}{\delta-2}\left[k_{i}-\left(r_{j+1}-l_{j+1}\right) T_{j+1}\right]}}{2(\Delta k)^{2}}\right.} \\
= & c_{i, j} .
\end{aligned}
$$

During each time step, we will then solve the tri-diagonal matrix system (5.11).

\subsubsection{Risk-Neutral Estimation by Solving PDE}

The parameters employed in our study are obtained by calibrating our leveraged Lévy model prices to market data. The forward PIDE formulation allows us to receive option prices for all maturities and all strikes after one execution of the PDE solver. The prices used in the calibration are those of all exchange traded strikes lying within $20 \%$ of the forward price on either side. The data is drawn from CRSP daily option data on $S \& P 500$ for December 31, 2003. The criterion for selection of the parameters is the minimization over the parameter space, $(\delta, \sigma)$, of the root mean square error on an equally weighted basis between market prices and model prices, specifically:

$$
\min _{\delta, \sigma} z=\sqrt{\frac{1}{n} \sum_{i=1}^{n}\left(\text { marketprice }_{i}-\text { modelprice }_{i}(\delta, \sigma)\right)^{2}} .
$$

The S\&P500 spot price on December 31, 2003 was 1110.595285. Market prices used in parameter estimation are for out-of-money options on account of 
their relative liquidity. More exactly, for strikes below the forward price we use put prices and for strikes above the forward price we use call prices. There are total 32 calls and 66 puts of 5 maturities. A summary of maturities we choose and the corresponding interest and dividend rates can be found in Table 5.6.

Table 5.6: Summary of $T, r$ and $q$ for $S \& P 500$ on December 31, 2003. Strikes vs. Option prices, $\star$ - Model prices, o - Market prices

\begin{tabular}{|c|c|c|c|}
\hline$S_{0}$ & $T$ & $r$ & $q$ \\
\hline 1110.595285 & 0.139350188 & 0.010861433 & 0.01615076 \\
1110.595285 & 0.215852921 & 0.011020496 & 0.016844507 \\
1110.595285 & 0.464372957 & 0.011821679 & 0.01599182 \\
1110.595285 & 0.713006837 & 0.012776774 & 0.015944081 \\
1110.595285 & 0.96175456 & 0.014074942 & 0.01647996 \\
\hline
\end{tabular}

The model prices are calculated on a fine mesh of both maturities and strikes and the ones that correspond to the strikes and maturities of the market prices are calculated using cubic spline interpolation. Table 5.7 contains the calibrated parameters. The fitted data plot is shown in Figure 5.2.

Table 5.7: Calibrated Parameters for S\&P500 on December 31, 2003

\begin{tabular}{|c|c|c|}
\hline$\delta$ & $\sigma$ & $z$ \\
\hline 1.6729 & 0.4849 & 1.4203 \\
\hline
\end{tabular}

The calibrated value for parameter $\delta$ is 1.6729 , which implies that $\alpha=-3.0572$ 
$\left(\delta=\frac{1}{\alpha}+2\right)$, negative $\alpha$ value indicates the leverage effect. However, in the context of diffusions, given their documented inability to address certain aspects of the density (e.g. long-tailedness and skewness), it is possible that estimated risk neutral leverage for example is just a reflection of well documented skewness and has little to do with volatilities actually moving with a market drop.

We now show some convergence results demonstrating numerical stability. In Table 5.8, we illustrate some examples of convergence as $\Delta T$ and $\Delta k$ approach zero. For all the options in the table, the maturity is $T=0.464372957$ and the prices shown there are all for call options. This result shows that the scheme is stable and convergent. 
Figure 5.2: Data Fitting For Calls of All Strikes and Maturities on 12-31-2003.

Strikes vs. Option prices, $\star$ - Model prices, o - Market prices

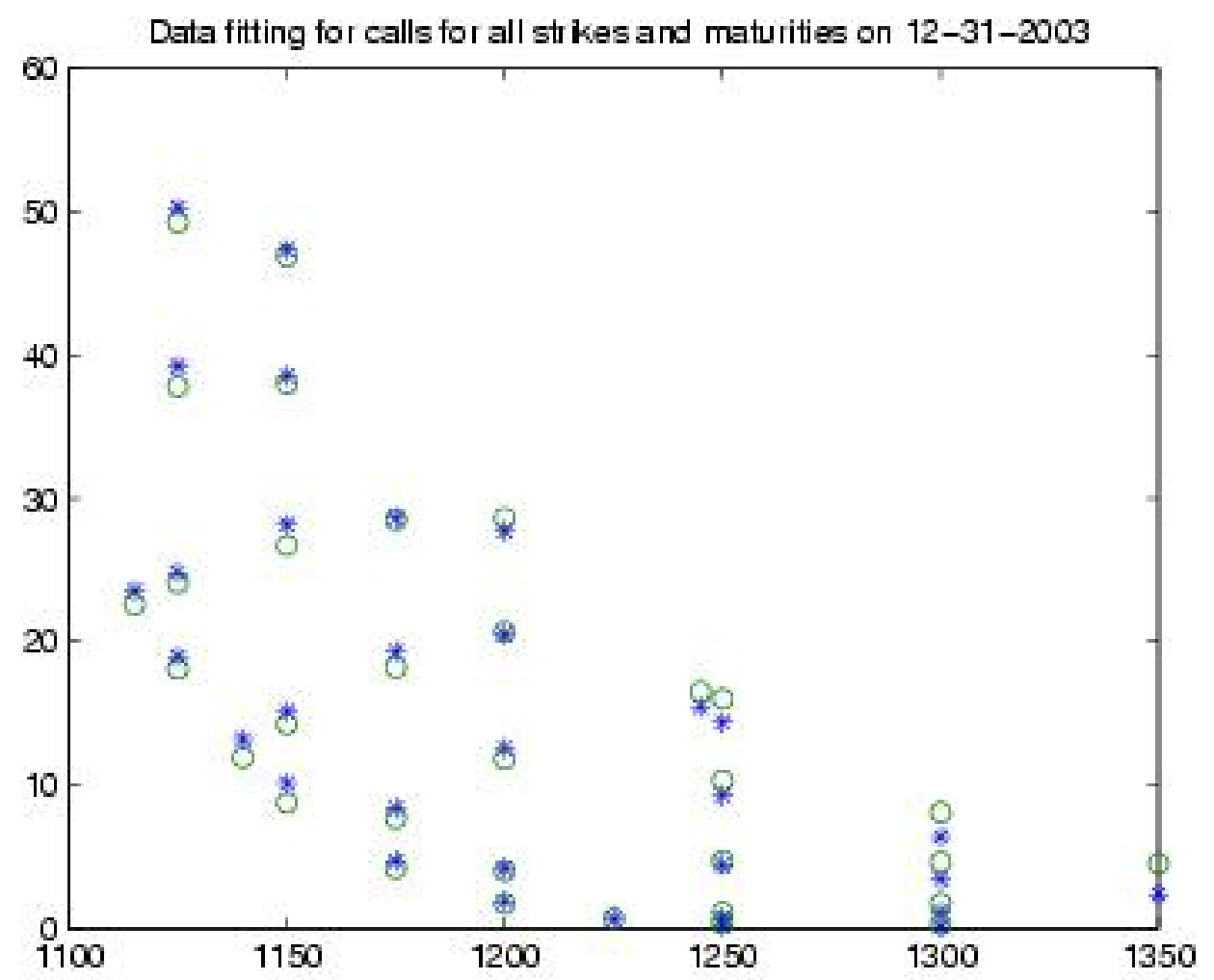


Table 5.8: Convergence results for maturity $T=0.464372957$ with various strikes.

\begin{tabular}{|c|c|c|c|c|c|}
\hline$K / M, N$ & 365,99 & 365,199 & 365,299 & 365,399 & 365,799 \\
\hline 1125 & 39.1534 & 39.1617 & 39.1637 & 39.1645 & 39.1679 \\
\hline 1150 & 28.1146 & 28.1253 & 28.1276 & 28.1285 & 28.1319 \\
\hline 1175 & 19.2731 & 19.2828 & 19.2848 & 19.2857 & 19.2887 \\
\hline 1200 & 12.5320 & 12.5377 & 12.5390 & 12.5395 & 12.5418 \\
\hline 1250 & 4.3969 & 4.3912 & 4.3904 & 4.3901 & 4.3908 \\
\hline 1300 & 1.1450 & 1.1345 & 1.1326 & 1.1319 & 1.1317 \\
\hline$K / M, N$ & 500,99 & 500,199 & 500,299 & 500,399 & 500,799 \\
\hline 1125 & 39.2099 & 39.2182 & 39.2202 & 39.2210 & 39.2244 \\
\hline 1150 & 28.1664 & 28.1770 & 28.1793 & 28.1803 & 28.1836 \\
\hline 1175 & 19.3170 & 19.32678 & 19.3289 & 19.3297 & 19.3326 \\
\hline 1200 & 12.5663 & 12.5721 & 12.5734 & 12.5739 & 12.5762 \\
\hline 1250 & 4.4121 & 4.4066 & 4.4057 & 4.4054 & 4.4061 \\
\hline 1300 & 1.1487 & 1.1382 & 1.1363 & 1.1356 & 1.1354 \\
\hline$K / M, N$ & 1000,99 & 1000,199 & 1000,299 & 1000, 399 & 1000,799 \\
\hline 1125 & 39.2228 & 39.2311 & 39.2330 & 39.2338 & 39.2372 \\
\hline 1150 & 28.1772 & 28.1878 & 28.1901 & 28.1911 & 28.1944 \\
\hline 1175 & 19.3244 & 19.3342 & 19.3363 & 19.3371 & 19.3401 \\
\hline 1200 & 12.5697 & 12.5755 & 12.5768 & 12.5773 & 12.5796 \\
\hline 1250 & 4.4090 & 4.4035 & 4.4026 & 4.4024 & 4.4031 \\
\hline 1300 & 1.1444 & 1.1339 & 1.1320 & 1.1313 & 1.1311 \\
\hline
\end{tabular}




\section{Chapter 6}

\section{THE DISCRETE CASE}

In this chapter we consider pure jump Lévy processes as our building blocks for constructing the Leveraged Lévy model. It is argued in [11] that the use of a jump process with infinite activity, i.e. one allowing infinitely many jumps in any time interval, effectively subsumes the need for an additional diffusion component. We replace Brownian motion with a Lévy process running at what we call the local speed function and this local speed function is still a deterministic function of the level of the stock price and time. The Lévy process involved in this chapter is the CGMY process which was introduced in the 2002 paper by Carr, Geman, Madan and Yor $([11])$. The parameters in the model are estimated using the Generalized Methods of Moment (GMM). A more complicated forward PIDE for options pricing under risk-neutral condition is developed and used to calibrate the parameters to the same option data used in Chapter 5. We conclude that this model with only exposure to pure jumps fits the data much better than the model in Chapter 5 where the uncertainty is modeled by Brownian motion.

\subsection{The Model}

The growth rate for the stock is $\mu$ and we suggest that the local absolute

volatility be of the form $\sigma S_{t}^{1+\alpha} e^{-\alpha \mu t}$. We would also like $S_{t} e^{-\mu t}$ to be a positive 
martingale. We incorporate the change in volatility using time changes and begin with a base Lévy process (here we are considering the pure jump case) with the property that $\exp (Z(t)+\omega t)$ is a martingale. Our proposed model for the stock price process is

$$
S_{t} e^{-\mu t}=\exp \left(Z\left(\int_{0}^{t} \sigma^{2} S_{u}^{2 \alpha} e^{-2 \alpha \mu u} d u\right)+\omega \int_{0}^{t} \sigma^{2} S_{u}{ }^{2 \alpha} e^{-2 \alpha \mu u} d u\right) .
$$

We now relate to Lamperti processes by defining the time change

$$
\zeta(t)=\int_{0}^{t} \sigma^{2} S_{u}^{2 \alpha} e^{-2 \alpha \mu u} d u
$$

Let $\eta(t)=\inf \{s \mid \zeta(s) \geq t\}$, and we have

$$
t=\int_{0}^{\eta(t)} \sigma^{2} S_{u}^{2 \alpha} e^{-2 \alpha \mu u} d u
$$

Evaluating equation (6.1) at $\eta(t)$ we obtain that

$$
S_{\eta(t)} e^{-\mu \eta(t)}=\exp (Z(t)+\omega t) .
$$

We also recognize from differentiating (6.2) that

$$
\begin{aligned}
\eta^{\prime}(t) & =\frac{1}{\sigma^{2}} S_{\eta(t)}^{-2 \alpha} e^{2 \alpha \mu \eta(t)} \\
& =\frac{1}{\sigma^{2}}\left[S_{\eta(t)} e^{-\mu \eta(t)}\right]^{-2 \alpha} \\
& =\frac{1}{\sigma^{2}} \exp (-2 \alpha Z(t)-2 \alpha \omega t) .
\end{aligned}
$$

Integrating both sides we have that (noticing $\eta(0)=0$ )

$$
\eta(t)=\frac{1}{\sigma^{2}} \int_{0}^{t} \exp (-2 \alpha Z(s)-2 \alpha \omega s) d s .
$$


Let $\beta=-\frac{1}{2 \alpha}^{1}$, and obviously $\beta \neq 0$ which we shall be aware throughout the rest of the chapter. Let the Lévy process $\xi^{(\beta)}(t)$ be

$$
\xi^{(\beta)}(t)=\frac{1}{\beta}(Z(t)+\omega t)
$$

Also now

$$
\sigma^{2} \eta(t)=\int_{0}^{t} \exp \left(\frac{1}{\beta}(Z(s)+\omega s) d s\right.
$$

From the Lamperti representation we have that

$$
\exp \left(\xi^{(\beta)}(t)\right)=L^{\left(\xi^{(\beta)}(t)\right)}\left(\sigma^{2} \eta(t)\right)
$$

But from (6.3) we have

$$
\begin{aligned}
S_{\eta(t)}^{1 / \beta} e^{-\mu \eta(t) / \beta} & =\exp \left(\frac{1}{\beta}(Z(t)+\omega t)\right. \\
& =\exp \left(\xi^{(\beta)}(t)\right) \\
& =L^{\left(\xi^{(\beta)}(t)\right)}\left(\sigma^{2} \eta(t)\right) .
\end{aligned}
$$

It follows that

$$
S_{t} e^{-\mu t}=\left[L^{\left(\xi^{(\beta)}(t)\right)}\left(\sigma^{2} t\right)\right]^{\beta}
$$

Noticing the scaling property of Lamperti processes, hence the general model for the stock price is

$$
S_{t}=S_{0} e^{\mu t} \sigma^{2 \beta}\left[L^{\left(\xi^{(\beta)}(t)\right)}(t)\right]^{\beta} .
$$

In the next section, we derive the infinitesimal generator of $S_{t}$ which will be of important use in our empirical investigation.

\footnotetext{
${ }^{1}$ Note that we exclude $\alpha=0$ which is the constant relative volatility case, without loss of generality under our emphasis of the setup of the Model
} 


\subsection{Deriving the Infinitesimal Generator for $S_{t}$}

Our model for the stock price is basically presenting $S_{t}$ as a Lamperti process to some power:

$$
S_{t}=S_{0} e^{\mu t} \sigma^{2 \beta}[L(t)]^{\beta}
$$

where $L(t)$ is the Lamperti process associated with a Lévy process, which we now denote as $\xi(t)$. However, in this pure jump case we no longer know what the Lamperti process is specifically ${ }^{2}$, our knowledge of the model is solely based on the knowledge of the underlying Lévy and what is given in the model construction. Now let us begin to derive the infinitesimal generator of $S$ from the generator of $\xi$. The infinitesimal generator will then be used in the context of conditional expectation to help set up both the GMM estimation and the forward equation for option pricing. Although the process is nontrivial, it is accessible given the knowledge of the underlying Lévy and the model connection.

Assume the Lévy triplet for $\xi(t)$ is $\left(a, 0, k_{\xi}(x) d x\right)$, where $a$ is the drift coefficient associated with a certain truncation function. According to a theorem due to Ito and Neveu (through applying results about Fourier and Fourier inversion transformation, and applying the Lévy-Khinchine formula, see [3] Page 139 for detail),

\footnotetext{
${ }^{2}$ In Chapter 5 we incorporate the BESQ process as the continuous Lamperti processes since BESQ is the only family of such processes and we know very well the law and other properties of BESQ process. Here in the pure jump case, we have no longer this knowledge. Dr. Madan and Dr. Yor attempted to study the characteristics of the associated Lamperti, but only came to an unsolved iterative equation.
} 
the infinitesimal generator of $\xi(t)$ is

$$
I_{\xi} f(\xi)=a f^{\prime}(\xi)+\int_{R-\{0\}}\left[f(\xi+\eta)-f(\xi)-f^{\prime}(\xi) h(\eta)\right] k_{\xi}(\eta) d \eta .
$$

From the result in 1994 paper $([71])$ we know that $I_{L}$, the generator of the associated Lamperti $L(t)$ is related to $I_{\xi}$ in the following way

$$
I_{L} f(x)=\frac{1}{x} I_{\xi}(f \circ \exp )(\ln x)
$$

Hence, we see that

$$
I_{L} f(x)=\frac{1}{x}\left[a x f^{\prime}(x)+\int_{R-\{0\}}\left[f\left(x e^{\eta}\right)-f(x)-x f^{\prime}(x) h(\eta)\right] k_{\xi}(\eta) d \eta\right] .
$$

We can now calculate the generator of $S_{t}$ via the following computation:

$$
\begin{aligned}
& E\left[f\left(S_{t}\right)-f\left(S_{0}\right)\right] \\
= & E\left[f\left(S_{0} e^{\mu t} \sigma^{2 \beta} L_{t}^{\beta}\right)-f\left(S_{0}\right)\right] \\
= & E\left[\int_{0}^{t} f^{\prime}\left(S_{u-}\right) \mu S_{u-} d u+\int_{0}^{t} I_{L}\left(f\left(S_{0} e^{\mu u} \sigma^{2 \beta} L_{u}^{\beta}\right)\right) d u\right] .
\end{aligned}
$$

We now work on the second term

$$
\begin{aligned}
& I_{L}\left(f\left(S_{0} e^{\mu u} \sigma^{2 \beta} L_{u}^{\beta}\right)\right) \\
= & \frac{1}{L}\left[a L \frac{\partial}{\partial L} f\left(S_{0} e^{\mu u} \sigma^{2 \beta} L_{u}^{\beta}\right)+\int_{R-\{0\}}\left[f\left(S_{0} e^{\mu u} \sigma^{2 \beta}\left(L_{u} e^{\eta}\right)^{\beta}\right)\right.\right. \\
& \left.\left.-f\left(S_{0} e^{\mu u} \sigma^{2 \beta} L_{u}^{\beta}\right)-L \frac{\partial}{\partial L} f\left(S_{0} e^{\mu u} \sigma^{2 \beta} L_{u}^{\beta}\right) h(\eta)\right] k_{\xi}(\eta) d \eta\right],
\end{aligned}
$$

the term

$$
\begin{aligned}
\frac{\partial}{\partial L} f\left(S_{0} e^{\mu u} \sigma^{2 \beta} L_{u}^{\beta}\right) & =f^{\prime}\left(S_{u}\right) S_{0} e^{\mu u} \sigma^{2 \beta} \beta L_{u}^{\beta-1} \\
& =f^{\prime}\left(S_{u}\right) \frac{S_{u} \beta}{L}
\end{aligned}
$$


or equivalently,

$$
L \frac{\partial}{\partial L} f\left(S_{0} e^{\mu u} \sigma^{2 \beta} L_{u}^{\beta}\right)=\beta S_{u} f^{\prime}\left(S_{u}\right)
$$

We also have from $S_{u}=S_{0} e^{\mu u} \sigma^{2 \beta} L_{u}^{\beta}$, that $L_{u}=\left(\frac{S_{u}}{S_{0} e^{\mu u} \sigma^{2 \beta}}\right)^{1 / \beta}$. We may now write (6.7) in terms of $S$

$$
\begin{aligned}
& I_{L}\left(f\left(S_{0} e^{\mu u} \sigma^{2 \beta} L_{u}^{\beta}\right)\right) \\
= & \left(\frac{S_{u}}{S_{0} e^{\mu u} \sigma^{2 \beta}}\right)^{-1 / \beta}\left[a \beta S_{u} f^{\prime}\left(S_{u}\right)\right. \\
& \left.+\int_{R-\{0\}}\left[f\left(S_{u-} e^{\beta \eta}\right)-f\left(S_{u-}\right)-\beta S_{u-} f^{\prime}\left(S_{u-}\right) h(\eta)\right] k_{\xi}(\eta) d \eta\right] .
\end{aligned}
$$

Substituting back in (6.6), we get

$$
\begin{aligned}
& E\left[f\left(S_{t}\right)-f\left(S_{0}\right)\right] \\
= & E\left[\int_{0}^{t} \mu S_{u-} f^{\prime}\left(S_{u-}\right) d u+\int_{0}^{t} d u\left(\frac{S_{u}}{S_{0} e^{\mu u} \sigma^{2 \beta}}\right)^{-1 / \beta}\left[a \beta S_{u} f^{\prime}\left(S_{u}\right)+\right.\right. \\
& \left.\left.\int_{R-\{0\}}\left[f\left(S_{u-} e^{\beta \eta}\right)-f\left(S_{u-}\right)-\beta S_{u-} f^{\prime}\left(S_{u-}\right) h(\eta)\right] k_{\xi}(\eta) d \eta\right]\right] .
\end{aligned}
$$

We know it is also true that

$$
E\left[f\left(S_{t}\right)-f\left(S_{0}\right)\right]=E\left[\int_{0}^{t} I_{S}(f)\left(S_{u}\right) d u\right]
$$

so it follows that

$$
\begin{aligned}
I_{S}(f)(x)= & \mu x f^{\prime}(x)+\left(\frac{x}{S_{0} e^{\mu u} \sigma^{2 \beta}}\right)^{-1 / \beta}\left[a \beta x f^{\prime}(x)+\right. \\
& \left.\int_{R-\{0\}}\left[f\left(x e^{\beta \eta}\right)-f(x)-\beta x f^{\prime}(x) h(\eta)\right] k_{\xi}(\eta) d \eta\right] .
\end{aligned}
$$

By rearranging the terms, we write the infinitesimal generator of $S_{t}$ as 


$$
\begin{aligned}
I_{S}(f)(x, u)= & {\left[\mu+\left(\frac{x}{S_{0} e^{\mu u} \sigma^{2 \beta}}\right)^{-1 / \beta} a \beta\right] x f^{\prime}(x) } \\
& +\left(\frac{x}{S_{0} e^{\mu u} \sigma^{2 \beta}}\right)^{-1 / \beta} \int_{R-\{0\}}\left[f\left(x e^{\beta \eta}\right)-f(x)-\beta x f^{\prime}(x) h(\eta)\right] k_{\xi}(\eta) d \eta .
\end{aligned}
$$

With $q$ denoting the dividend yield, and changing $\mu$ to $\mu-q$ in $I_{S}$, we have the more general form

$$
\begin{aligned}
& I_{S}(f)(x, u) \\
= & {\left[(\mu-q)+\left(\frac{x}{S_{0} e^{(\mu-q) u} \sigma^{2 \beta}}\right)^{-1 / \beta} a \beta\right] x f^{\prime}(x) } \\
+ & \left(\frac{x}{S_{0} e^{(\mu-q) u} \sigma^{2 \beta}}\right)^{\frac{-1}{\beta}} \int_{R-\{0\}}\left[f\left(x e^{\beta \eta}\right)-f(x)-\beta x f^{\prime}(x) h(\eta)\right] k_{\xi}(\eta) d \eta .
\end{aligned}
$$

\subsection{GMM Estimation}

Generalized Methods of Moment (GMM) estimation is one of two developments in econometrics in the 80 s that revolutionized empirical work in macroeconomics. (The other being the understanding of unit roots and cointegration). The

path breaking articles on GMM were those of Hansen (1982, [33]) and Hansen and Singleton (1982, [35]).

GMM, is an econometric procedure for estimating the parameters of a model. Hansen (1982, [33]) developed GMM as an extension to the classical method of moments estimators dating back more than a century. The basic idea is to choose parameters of the model so as to match the moments of the model to those of the data as closely as possible. The moment conditions are chosen by the analyst based on the problem at hand. A weighting matrix determines the relative importance 
of matching each moment. Most common estimation procedures can be couched in this framework, including ordinary least squares, instrumental variables estimators, two-stage least squares, and in some cases maximum likelihood.

A key advantage to GMM over other estimation procedures is that the statistical assumptions required for hypothesis testing are quite weak. Of course, nothing comes for free. The cost is a loss of efficiency over methods such as Maximum Likelihood (MLE). One can view MLE as a limiting case of GMM: under MLE the distribution of errors is specified so in a sense all of the moments are incorporated. The trouble with MLE is often that the errors may not follow a known distribution (such as the Normal, which is almost the universal standard in MLE). Thus, GMM offers a compromise between the efficiency of MLE and robustness to deviations from normality (or other distributional forms). Also note that, except for some special cases, the GMM results are asymptotic. These are the reasons we are to use GMM to estimate the parameters in our model.

Due to the lack of knowledge of the law or characteristic function of our leveraged Lévy model, we implement the Generalized Methods of Moments (GMM) estimation to estimate our parameters.

\subsubsection{Introduction of GMM}

GMM chooses the parameters which minimize the quadratic

$$
J_{T}=\mathbf{m}(\theta)^{\prime} \mathbf{W} \mathbf{m}(\theta),
$$


where $\theta$ is a $k$-vector of parameters, $\mathbf{m}(\theta)$ is an $L$-vector of orthogonality conditions, and $\mathbf{W}$ is an $L \times L$ positive definite weighting matrix. The objective function has a least-squares flavor.

The moment conditions $\mathbf{m}(\theta)$ set means of functions of the data and parameters to zero. One simple restriction estimates the mean $\mu$ of data $y_{t}$

$$
E\left[y_{t}\right]=\mu,
$$

given the population orthogonality condition

$$
E\left[y_{t}-\mu\right]=0
$$

and sample counterpart

$$
\mathbf{m}(\theta)=\frac{1}{T} \sum_{t=1}^{T} y_{t}-\mu .
$$

Another restriction, on the variance $\left(\sigma^{2}\right)$, is

$$
E\left[\left(y_{t}-\mu\right)^{2}\right]=\sigma^{2},
$$

giving the system

$$
E\left[\begin{array}{c}
y_{t}-\mu \\
\left(y_{t}-\mu\right)^{2}-\sigma^{2}
\end{array}\right]=\left[\begin{array}{l}
0 \\
0
\end{array}\right] .
$$

Note that the moment condition for the mean is needed to estimate the variance.

Similarly, a covariance restriction would be

$$
E\left[\left(x_{t}-\mu_{x}\right)\left(y_{t}-\mu_{y}\right)\right]=\sigma_{x, y},
$$


giving the system

$$
E\left[\begin{array}{c}
x_{t}-\mu_{x} \\
y_{t}-\mu_{y} \\
\left(x_{t}-\mu_{x}\right)\left(y_{t}-\mu_{y}\right)-\sigma_{x, y}
\end{array}\right]=\left[\begin{array}{l}
0 \\
0 \\
0
\end{array}\right] .
$$

The terms $\mu_{x}, \mu_{y}, \sigma_{x}, \sigma_{y}$ and $\sigma_{x, y}$ are parameters we wish to estimate, whereas $x_{t}$ and $y_{t}$ are data.

A key ingredient to GMM is the specification of the moment, or orthogonality, conditions $\mathbf{m}(\theta)$. The moment conditions are commonly based on the error terms from an economic model. Consider a general model of the form

$$
\mathbf{y}_{[T \times 1]}=\mathbf{f}\left(\mathbf{X}_{[T \times k]} ; \theta\right)+\epsilon,
$$

where $\mathbf{f}$ can be a nonlinear function. We then will need $L \geq k$ (independent) restrictions in order to identify the $k$-vector of parameters, $\theta$. The moment conditions restrict unconditional means of the data to be zero. The population version of each of these restrictions $(l=1, \ldots, L)$ is of the form

$$
E\left[m_{l}(\mathbf{y}, \mathbf{X} ; \theta)\right]=0
$$

The sample analog is

$$
m_{l}(\mathbf{y}, \mathbf{X} ; \hat{\theta})=\frac{1}{T} \sum_{t=1}^{T} m_{l, t}\left(\mathbf{y}_{t}, \mathbf{x}_{t} ; \hat{\theta}\right)
$$

where $\mathbf{y}_{t}$ and $\mathbf{x}_{t}$ denote row $t$ of the matrices $\mathbf{y}$ and $\mathbf{X}$, transposed to be column vectors. Note that $\mathbf{m}_{t}$ (with the time subscript) indicates an observation-by-observation 
set of values, while $\mathbf{m}$ (no time subscript) indicates the moment (average) of the $\mathbf{m}_{t}^{\prime}$ s.

The moment conditions utilized, though somewhat arbitrary, are often guided by economic principles and the model of interest. For example, in finance the return on an asset of this period is generally modeled as unpredictable by (orthogonal to) information in prior periods, so moment conditions often incorporate past returns, interest rates, etc.

Note that there must be at least as many moment conditions as there are parameters to achieve identification. If you have too few restrictions, you can "create" more by using instruments. Returning to (6.10), suppose $E\left[\epsilon_{t} \mathbf{x}_{\mathbf{t}}\right] \neq \mathbf{0}$, but that $E\left[\epsilon_{t} \mathbf{z}_{\mathbf{t}}\right]=\mathbf{0}$. The $\mathbf{z}_{\mathbf{t}}$ 's are referred to as instruments. In sample, the model errors are

$$
\mathbf{e}(\hat{\theta})=\mathbf{y}-\mathbf{f}(\mathbf{X} ; \hat{\theta})
$$

giving the moment conditions

$$
\mathbf{m}(\theta)=\frac{1}{T} \sum_{t=1}^{T} \mathbf{z}_{\mathbf{t}} e\left(\mathbf{y}_{t}, \mathbf{x}_{t} ; \theta\right)=\frac{1}{T} \mathbf{Z}^{\prime} \mathbf{e}(\mathbf{y}, \mathbf{X} ; \hat{\theta})
$$

This can actually be generalized for simultaneous equations by letting $e_{t}$ represent the vector of residuals for each equation at time $t$, giving $\mathbf{m}_{t}=\epsilon_{t} \otimes \mathbf{z}_{t}$. The notation $\otimes$ indicates the Kroneker product, which multiplies every element of $\epsilon_{t}$ by $\mathbf{z}_{t}$.

This approach with the instruments changes the question of "which moments" to "which Z". It is common to include a constant as an instrument to restrict the 
model errors to have mean zero.

\section{Illustration of a simple example:}

Suppose we have a simple model $y_{t}=\alpha+x_{t} \beta+\epsilon_{t}$ and three observations of $\left\{x_{t}, y_{t}\right\}:\{0,1\},\{1,3\},\{2,5\}$. We need (at least) two moment conditions to identify $\alpha$ and $\beta$. The natural ones to choose are $E\left[\epsilon_{t}\right]=0$ and $E\left[x_{t} \epsilon_{t}\right]=0$, the normal equations from OLS. In this case, $z_{t}=\left[1 x_{t}\right]$. In sample,

$$
\mathbf{m}=\left[\begin{array}{c}
m_{1} \\
m_{2}
\end{array}\right]=\frac{1}{3} \sum_{t=1}^{3} \mathbf{z}_{t}^{\prime} e_{t}=\frac{1}{3} \sum_{t=1}^{3}\left[\begin{array}{c}
1 e_{t} \\
x_{t} e_{t}
\end{array}\right]=\frac{1}{3} \sum_{t=1}^{3}\left[\begin{array}{c}
1\left(y_{t}-\alpha-x_{t} \beta\right) \\
x_{t}\left(y_{t}-\alpha-x_{t} \beta\right)
\end{array}\right] .
$$

In this case, the objective function is minimized when $m=0$, or

$$
\begin{aligned}
m_{1} & =\frac{1}{3}[(1-\alpha-0 \beta)+(3-\alpha-1 \beta)+(5-\alpha-2 \beta)] \\
& =3-\alpha-\beta=0, \\
m_{2} & =\frac{1}{3}[0(1-\alpha-0 \beta)+1(3-\alpha-1 \beta)+2(5-\alpha-2 \beta)] \\
& =\frac{1}{3}(13-3 \alpha-5 \beta)=0 .
\end{aligned}
$$

Equation (6.13) gives $\alpha=3-\beta$, which can be substituted into (6.14) to get $\beta=2$, implying $\alpha=1$.

The above analysis ignored the presence of the weighting matrix $\mathbf{W}$ in the minimization of the objective function (assuming it is equal to the identity matrix). We will now consider the role of the weighting matrix.

If there are as many moment conditions as parameters, the moments will all be 
perfectly matched and the objective function $J_{T}$ in (6.9) will have a value of zero. This is referred to as the "just-identified" case. In the situation where there are more moment conditions than parameters ("over-identified") not all of the moment restrictions will be satisfied so a weighting matrix $\mathbf{W}$ determines the relative importance of the various moment conditions. An important contribution of Hansen (1982, [33]) is to point out that setting $\mathbf{W}=\mathbf{S}^{-1}$, the inverse of an asymptotic covariance matrix, is optimal in the sense that it yields $\hat{\theta}$ with the smallest asymptotic variance. Intuitively, more weight is given to the moment conditions with less uncertainty. $\mathbf{S}$ is also known as the spectral density matrix evaluated at frequency zero. There are many approaches for estimating $\mathbf{S}$ which can account for various forms of heteroskedasticity and/or serial correlation, including White (1980, [69]), the Bartlett kernel using by Newey and West (1987, [53]), the Parzen kernel of Gallant (1987, [29]), the truncated kernel of Hansen (1982, [33]) and Hansen and Hodrick (1980, [34]), or the "automatic" bandwidth selection from Andrews and Monahan (1992, [2]) with Quadratic-Spectral or Tukey-Hanning kernels. More details of theory of Generalized Method of Moments can be found in e.g. [32].

The Spectral Density Matrix for the kernel-based estimators ( White, Hansen, Newey-West and Gallant) is given by

$$
\hat{\mathbf{S}}=\hat{\mathbf{S}}_{0}+\sum_{j=1}^{J} w(j)\left[\hat{\mathbf{S}}_{j}+\hat{\mathbf{S}}_{j}^{\prime}\right]
$$

where 


$$
\begin{aligned}
\hat{\mathbf{S}}_{j} & =\frac{T}{T-k} \frac{1}{T} \sum_{t=j+1}^{T} \mathbf{m}_{t}(\hat{\theta}) \mathbf{m}_{t-j}(\hat{\theta})^{\prime} \\
& =\frac{1}{T-k} \sum_{t=j+1}^{T}\left[\epsilon_{t} \otimes \mathbf{z}_{t}\right]\left[\epsilon_{t-j} \otimes \mathbf{z}_{t-j}\right]^{\prime} \\
& =\frac{1}{T-k} \sum_{t=j+1}^{T}\left[\epsilon_{t} \epsilon_{t-j}^{\prime} \otimes \mathbf{z}_{t} \mathbf{z}_{t-j}^{\prime}\right] .
\end{aligned}
$$

The $\frac{T}{T-k}$ term is a small sample degrees of freedom correction. The term $w(j)$ is the kernel weight, and it is what distinguishes the various estimators. Terms beyond the lag truncation parameter $J$ are given weights of zero in kernel other than the Quadratic-Spectral and Tukey-Hanning.

In general, and "optimal" weighting matrix requires an estimate of the parameter vector, yet at the same time, estimating the parameters requires a weighting matrix. To solve this dependency, common practice is to set the initial weighting matrix to the identity, and then calculate the parameter estimates. A new weighting matrix is calculated with the last parameter estimates, then new parameter estimates with the updated weighting matrix.

$$
\begin{aligned}
\mathbf{W}_{0} & =\mathbf{I} \\
\hat{\theta}_{1} & =\operatorname{argmin}\left[\mathbf{m}(\theta)^{\prime} \mathbf{W}_{0} \mathbf{m}(\theta)\right], \\
\mathbf{W}_{1} & =f\left(\hat{\theta}_{1}\right), \\
\hat{\theta}_{2} & =\operatorname{argmin}\left[\mathbf{m}(\theta)^{\prime} \mathbf{W}_{1} \mathbf{m}(\theta)\right] .
\end{aligned}
$$

The process can then be iterated further by calculating $\mathbf{W}_{2}$ then minimizing to find 
$\hat{\theta}_{3}$ and so on. In general, iterating to end with $\hat{\theta}_{n}$ is called n-stage GMM. You can also iterate until the change in objective function is sufficiently small.

\subsubsection{Using GMM in CGMY case}

We set $X_{t}=\ln S_{t}$ to be the log price. We know that for any function $f$

$$
\begin{aligned}
E_{t}\left[f\left(X_{t+h}\right)-f\left(X_{t}\right)\right] & =E_{t}\left[\int_{t}^{t+h} I_{X}(f)\left(X_{u}, u\right) d u\right] \\
& \approx E_{t}\left[h I_{x}(f)\left(X_{t}, t\right)\right]
\end{aligned}
$$

where $I_{X}(f)\left(X_{t}, t\right)$ is the infinitesimal generator of $X_{t}$ which, from Section 6.5 we know as

$$
I_{X}(f)(x, u)=a(x, u) \frac{\partial}{\partial x} f+\int_{R-\{0\}}\left[f(x+\eta)-f(x)-\eta \frac{\partial}{\partial x} f\right] k(x, u, \eta) d \eta
$$

where

$$
\begin{gathered}
a(x, u)=\mu+a \beta\left(\frac{e^{x}}{S_{0} e^{\mu u} \sigma^{2 \beta}}\right)^{-\frac{1}{\beta}}, \\
k(x, u, \nu)=\left(\frac{e^{x}}{S_{0} e^{\mu u} \sigma^{2 \beta}}\right)^{-\frac{1}{\beta}} k_{C G M Y}(\nu) .
\end{gathered}
$$

Recall $a=\frac{\gamma+\omega}{\beta}$, and $\gamma+\omega=-\int_{R-\{0\}}\left(e^{\nu}-1-\nu\right) k_{C G M Y}(\nu) d \nu$.

To use the GMM procedure, we provide the orthogonality conditions for different choices of $Z_{t}$ as

$$
E\left[\left(f\left(X_{t+h}\right)-f\left(X_{t}\right)-h I_{X}(f)\left(X_{t}, t\right)\right) Z_{t}\right]=0 .
$$


which will be replaced in the GMM procedure by its sample counterpart. $Z_{t}$ are taken to be instruments known at time $t$, we use constant C, and $S_{t}, S_{t}-S_{t-1}$, $\left(S_{t}-S_{t-1}\right)^{2}, S_{t}-2 S_{t-1}+S_{t-2},\left(S_{t}-2 S_{t-1}+S_{t-2}\right)^{2}$

We take the function $f$ to be

$$
f(x)=e^{-\lambda\left(x-\ln S_{0}\right)}=S_{0}^{\lambda} e^{-\lambda x}
$$

for some chosen $\lambda$. So then

$$
f^{\prime}(x)=-\lambda S_{0}^{\lambda} e^{-\lambda x}
$$

The function $f$, by construction, acts like a Laplace transform on scaled log price of stock. Since $\ln \frac{S}{S_{0}}$ will be in a range of 1 , we choose $\lambda$ to be between 0.25 and 2 with step-size 0.25 . We have 8 functions and 6 instruments yielding 48 orthogonalities to be tested simultaneously.

Now we should compute $\left.I_{X}(f)\left(X_{t}, t\right)\right)$ :

$$
\begin{aligned}
& I_{X}(f)\left(X_{t}, t\right) \\
= & (-\lambda) S_{0}^{\lambda}\left[\mu+(\gamma+\omega)\left(\frac{S_{t}}{S_{0} e^{\mu t} \sigma^{2 \beta}}\right)^{-\frac{1}{\beta}}\right] e^{-\lambda X_{t}} \\
& +C S_{0}^{\lambda}\left(\frac{S_{t}}{S_{0} e^{\mu t} \sigma^{2 \beta}}\right)^{-\frac{1}{\beta}} \int_{R-\{0\}}\left[e^{-\lambda\left(X_{t}+\eta\right)}-e^{-\lambda X_{t}}+\lambda \eta e^{-\lambda X_{t}}\right] \frac{e^{A \eta-B|\eta|}}{|\eta|^{1+Y}} d \eta \\
= & (-\lambda) S_{0}^{\lambda}\left[\mu+(\gamma+\omega)\left(\frac{S_{t}}{S_{0} e^{\mu t} \sigma^{2 \beta}}\right)^{-\frac{1}{\beta}}\right] e^{-\lambda X_{t}}+C S_{0}^{\lambda}\left(\frac{S_{t}}{S_{0} e^{\mu t} \sigma^{2 \beta}}\right)^{-\frac{1}{\beta}} e^{-\lambda X_{t}} \phi(\lambda),
\end{aligned}
$$

where $\phi(\lambda)$ is

$$
\phi(\lambda)=\int_{R-\{0\}}\left[e^{-\lambda \eta}-1+\lambda \eta\right] \frac{e^{A \eta-B|\eta|}}{|\eta|^{1+Y}} d \eta
$$


We see that $\phi(\lambda)$ is related to $\gamma+\omega=-\int_{R-\{0\}}\left(e^{\nu}-1-\nu\right) k_{C G M Y}(\nu) d \nu$. In fact, $\gamma+\omega=-C \phi(-1)$. Analogous to the evaluation of $\gamma+\omega$ in the Appendix and by knowing the value

$$
\begin{aligned}
& \gamma+\omega= \\
& \left\{\begin{array}{c}
C\left[\Gamma(1-Y)\left(M^{Y-1}-G^{Y-1}\right)-\Gamma(-Y)\left[(M-1)^{Y}-M^{Y}+(G+1)^{Y}-G^{Y}\right]\right] \\
Y<0 ; \\
C \Gamma(1-Y)\left[M^{Y-1}-G^{Y-1}+\frac{(M-1)^{Y}}{Y}-\frac{M^{Y}}{Y}+\frac{(G+1)^{Y}}{Y}-\frac{G^{Y}}{Y}\right] \\
0<Y<1 ; \\
C \frac{\Gamma(2-Y)}{Y(1-Y)}\left[(M-1)^{Y}-M^{Y}+(G+1)^{Y}-G^{Y}+Y M^{Y-1}-Y G^{Y-1}\right], \\
1<Y<2 .
\end{array}\right.
\end{aligned}
$$

as well as

$$
\begin{aligned}
\gamma & =\int_{R-\{0\}} h(x) k_{C G M Y}(x) d x \\
& =\int_{R-\{0\}} x k_{C G M Y}(x) d x \\
& =\left\{\begin{array}{c}
C \Gamma(1-Y)\left(M^{Y-1}-G^{Y-1}\right), Y<1, \\
\frac{C \Gamma(2-Y)}{1-Y}\left(M^{Y-1}-G^{Y-1}\right), Y>1 .
\end{array}\right.
\end{aligned}
$$

We can evaluate $\phi(\lambda)$ as: 


$$
\begin{aligned}
& \phi(\lambda)= \\
& \left\{\begin{array}{c}
\lambda \Gamma(1-Y)\left(M^{Y-1}-G^{Y-1}\right)+\Gamma(-Y)\left[(M+\lambda)^{Y}-M^{Y}+(G-\lambda)^{Y}-G^{Y}\right], \\
Y<0 ; \\
\lambda \Gamma(1-Y)\left(M^{Y-1}-G^{Y-1}\right)-\frac{\Gamma(1-Y)}{Y}\left[(M+\lambda)^{Y}-M^{Y}+(G-\lambda)^{Y}-G^{Y}\right], \\
0<Y<1 ; \\
\frac{\lambda \Gamma(2-Y)}{(1-Y)}\left(M^{Y-1}-G^{Y-1}\right)-\frac{\Gamma(2-Y)}{Y(1-Y)}\left[(M+\lambda)^{Y}-M^{Y}+(G-\lambda)^{Y}-G^{Y}\right], \\
1<Y<2 .
\end{array}\right.
\end{aligned}
$$

Putting the results together, in GMM condition (6.15) we have,

$$
\begin{aligned}
& f\left(X_{t+h}\right)-f\left(X_{t}\right)-h I_{X}(f)\left(X_{t}, t\right) \\
= & S_{0}{ }^{\lambda} e^{-\lambda X_{t+h}}-S_{0}{ }^{\lambda} e^{-\lambda X_{t}} \\
& -h S_{0}{ }^{\lambda} e^{-\lambda X_{t}}\left[-\lambda \mu+\left(\frac{S_{t}}{S_{0} e^{\mu t} \sigma^{2 \beta}}\right)^{-\frac{1}{\beta}}(C \phi(\lambda)-\lambda(\gamma+\omega))\right] .
\end{aligned}
$$

\subsubsection{Estimation Results}

We take our data from the CRSP indices daily data of S\&P500 from Jan. 4th, 1993 to Dec. 31st, 2004. The GMM experiment is carried out in Matlab using a package written by Dr. Michael T. Chiff from Krannert Graduate School of Management. In the "just-identified" case, where there are as many moment conditions as parameters, we use $\lambda=0.25,0.5,0.75,1.0,1.25,1.5,1.75$ as the seven values to account for the variation of function $f$ and only the constant as the instrument. In this situation the choice of weighting matrix is irrelevant and so we simply use the identity matrix, since the moments will all be perfectly matched and the objective 
function $J_{T}$ in $(6.9)$ will have a value of zero. In fact, the weighting matrix is set to be $\left(\mathbf{I}_{N} \otimes \mathbf{Z}^{\prime} \mathbf{Z}\right)^{-1}$ to incorporate the magnitudes of the instruments, in order to deal with the issue of estimation being sensitive to scaling of the data when using identity matrix as the weighting matrix. The weights attached to moments are shown in Table 6.1. The result of parameter estimation is shown in Table 6.2.

Table 6.1: Weights Attached to Moments When $\mathbf{W}=\left(\mathbf{I}_{N} \otimes \mathbf{Z}^{\prime} \mathbf{Z}\right)^{-1}$ in the Justidentified Case

\begin{tabular}{|c|c|c|c|c|c|c|c|}
\hline Moment & 1 & 2 & 3 & 4 & 5 & 6 & 7 \\
\hline $\mathrm{C}$ & 0.0310 & 0.0657 & 0.1025 & 0.1407 & 0.1798 & 0.2197 & 0.2606 \\
$\mathrm{G}$ & 0.0305 & 0.0649 & 0.1016 & 0.1400 & 0.1797 & 0.2206 & 0.2627 \\
$\mathrm{M}$ & 0.0315 & 0.0665 & 0.1034 & 0.1413 & 0.1798 & 0.2189 & 0.2586 \\
$\mathrm{Y}$ & 0.0310 & 0.0657 & 0.1025 & 0.1407 & 0.1798 & 0.2197 & 0.2606 \\
$\mu$ & 0.0353 & 0.0755 & 0.1150 & 0.1511 & 0.1825 & 0.2092 & 0.2313 \\
$\sigma$ & 0.0310 & 0.0657 & 0.1025 & 0.1407 & 0.1798 & 0.2197 & 0.2606 \\
$\beta$ & 0.0452 & 0.0877 & 0.1248 & 0.1553 & 0.1793 & 0.1974 & 0.2103 \\
\hline
\end{tabular}

Table 6.2: GMM Estimation of Parameters in Just-identified Case

\begin{tabular}{|c|c|c|c|c|c|c|c|}
\hline & $C$ & $G$ & $M$ & $Y$ & $\mu$ & $\sigma$ & $\beta$ \\
\hline Estimates & 20.0417 & 36.3878 & 36.8605 & 1.1987 & 0.0299 & 0.6747 & 1.0042 \\
\hline S.E. & 0.1090 & 0.0477 & 0.0498 & 0.0121 & 0.0010 & 0.0080 & 0.0066 \\
\hline
\end{tabular}

In the "over-identified" situation, where there are more moment conditions 
than parameters, we use eight equations setting $\lambda=0.25,0.5,0.75,1.0,1.25$, 1.5, 1.75, 2.0, and the instruments $Z_{t}$ are taken to be constant C, $S_{t}, S_{t}-S_{t-1}$, $\left(S_{t}-S_{t-1}\right)^{2}, S_{t}-2 S_{t-1}+S_{t-2},\left(S_{t}-2 S_{t-1}+S_{t-2}\right)^{2}$. Hence there are 48 moment conditions and 7 parameters. The weighting matrix of initial iteration is set to be $\mathbf{W}_{0}=\left(\mathbf{I}_{N} \otimes \mathbf{Z}^{\prime} \mathbf{Z}\right)^{-1}$. In the following iterations the "Newey-West" method ([53]) is used to calculate the spectral density matrix. The final minimized objective function has value of 0.0141 . The estimation result is summarized in Table 6.3 and other output is as following.

We gladly observe that in both cases the estimates $\beta$ show leverage effect as we expect. In the just-identified case, $\beta=1.0042$ implies $\alpha=-0.4979$ while in the over-identified case, $\beta=3.5047$ which implies $\alpha=-0.1427$. Both values are negative hence admits a negative relation between local volatility and spot price.

\section{GMM ESTIMATION PROGRAM}

7 Parameters, 48 Moment Conditions

8 Equation Model, 6 Instruments

3021 Observations

2 Passes, Max., 100 Iterations/Pass

Search Direction: $\quad$ Marquardt (lambda $>=0.01$ ) 
Derivatives: $\quad$ Numerical

Initial Weighting Matrix: inv(Z'Z)

Weighting Matrix: Optimal

Spectral Density Matrix: Newey-West (12 lags)

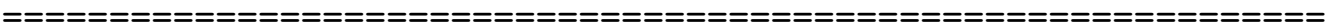

\begin{tabular}{|c|c|c|c|c|c|}
\hline & & Moment & Std Err & t-stat & $\mathrm{p}-\mathrm{val}$ \\
\hline Moment & 1 & 0.000041 & 0.000038 & 1.06 & 0.2892 \\
\hline Moment & 2 & 0.059542 & 0.039329 & 1.51 & 0.1300 \\
\hline Moment & 3 & 0.000276 & 0.000476 & 0.58 & 0.5618 \\
\hline Moment & 4 & -0.035500 & 0.024291 & -1.46 & 0.1439 \\
\hline Moment & 5 & -0.000421 & 0.000763 & -0.55 & 0.5814 \\
\hline Moment & 6 & -0.011596 & 0.048000 & -0.24 & 0.8091 \\
\hline Moment & 7 & 0.000060 & 0.000063 & 0.95 & 0.3441 \\
\hline Moment & 8 & 0.087927 & 0.062781 & 1.40 & 0.1614 \\
\hline Moment & 9 & 0.000430 & 0.000768 & 0.56 & 0.5750 \\
\hline Moment & 10 & -0.056959 & 0.038882 & -1.46 & 0.1429 \\
\hline Moment & 11 & -0.000624 & 0.001217 & -0.51 & 0.6080 \\
\hline Moment & 12 & -0.018815 & 0.073939 & -0.25 & 0.7991 \\
\hline Moment & 13 & 0.000066 & 0.000078 & 0.84 & 0.4029 \\
\hline
\end{tabular}




\begin{tabular}{|c|c|c|c|c|c|}
\hline Moment & 14 & 0.097007 & 0.075440 & 1.29 & 0.1985 \\
\hline Moment & 15 & 0.000499 & 0.000933 & 0.53 & 0.5930 \\
\hline Moment & 16 & -0.068758 & 0.046892 & -1.47 & 0.1426 \\
\hline Moment & 17 & -0.000694 & 0.001462 & -0.47 & 0.6349 \\
\hline Moment & 18 & -0.023223 & 0.085789 & -0.27 & 0.7866 \\
\hline Moment & 19 & 0.000064 & 0.000087 & 0.73 & 0.4645 \\
\hline Moment & 20 & 0.094686 & 0.080877 & 1.17 & 0.2417 \\
\hline Moment & 21 & 0.000508 & 0.001013 & 0.50 & 0.6163 \\
\hline Moment & 22 & -0.074010 & 0.050487 & -1.47 & 0.1427 \\
\hline Moment & 23 & -0.000687 & 0.001571 & -0.44 & 0.6618 \\
\hline Moment & 24 & -0.025804 & 0.088897 & -0.29 & 0.7716 \\
\hline Moment & 25 & 0.000058 & 0.000091 & 0.63 & 0.5277 \\
\hline Moment & 26 & 0.086141 & 0.081590 & 1.06 & 0.2911 \\
\hline Moment & 27 & 0.000477 & 0.001035 & 0.46 & 0.6451 \\
\hline Moment & 28 & -0.074914 & 0.051164 & -1.46 & 0.1431 \\
\hline Moment & 29 & -0.000638 & 0.001590 & -0.40 & 0.6883 \\
\hline Moment & 30 & -0.027174 & 0.086798 & -0.31 & 0.7542 \\
\hline Moment & 31 & 0.000050 & 0.000093 & 0.54 & 0.5911 \\
\hline Moment & 32 & 0.074685 & 0.079331 & 0.94 & 0.3465 \\
\hline Moment & 33 & 0.000421 & 0.001021 & 0.41 & 0.6800 \\
\hline Moment & 34 & -0.073018 & 0.049961 & -1.46 & 0.1439 \\
\hline Moment & 35 & -0.000569 & 0.001554 & -0.37 & 0.7141 \\
\hline Moment & 36 & -0.027719 & 0.081798 & -0.34 & 0.7347 \\
\hline
\end{tabular}




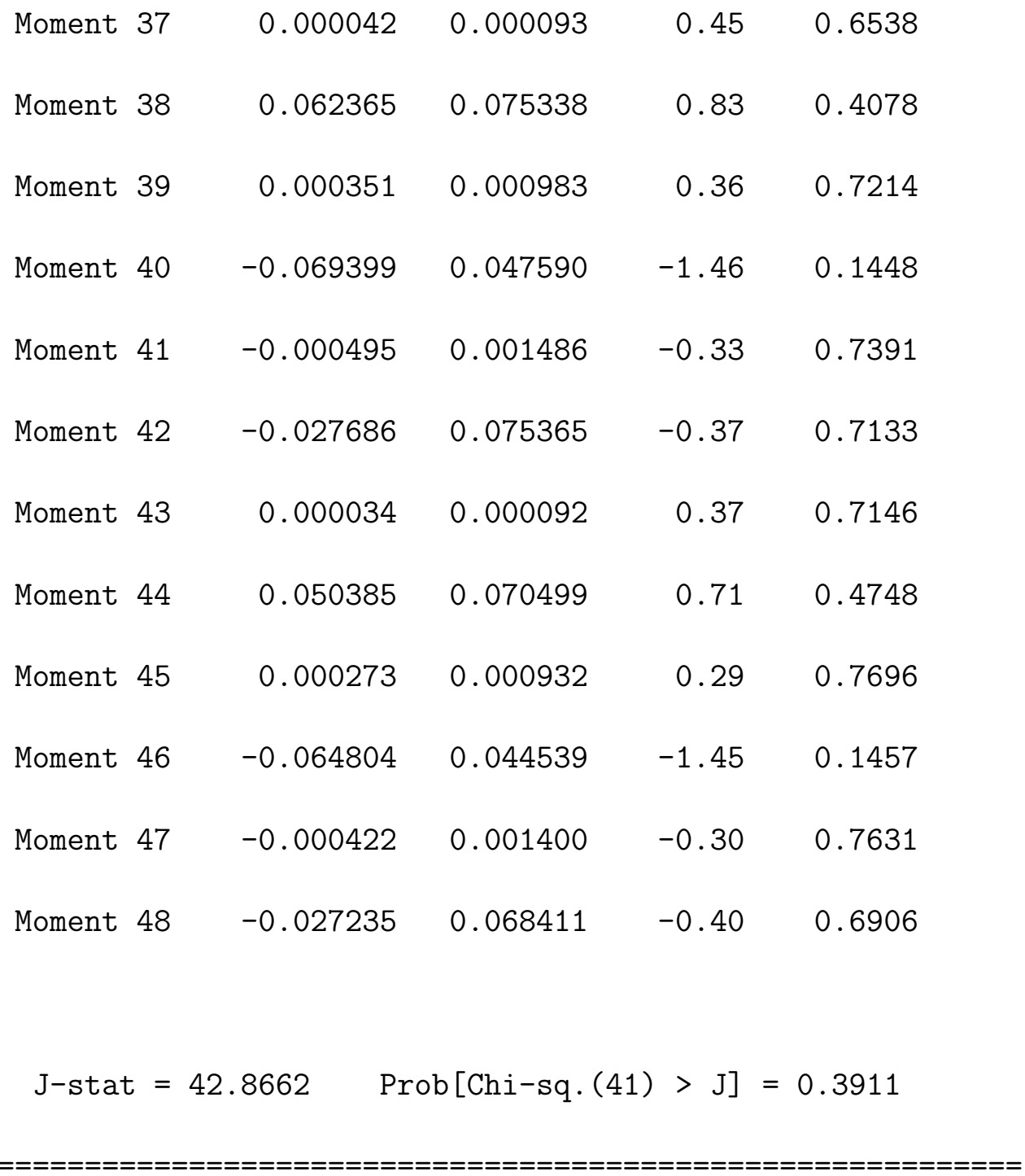

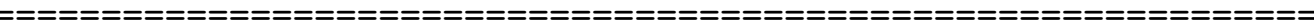

Table 6.3: GMM Estimation of Parameters in Over-identified Case

\begin{tabular}{|c|c|c|c|c|c|c|c|}
\hline & $C$ & $G$ & $M$ & $Y$ & $\mu$ & $\sigma$ & $\beta$ \\
\hline Estimates & 20.0372 & 36.5764 & 36.6558 & 0.4810 & 0.0626 & 0.4953 & 3.5047 \\
S.E. & 0.3840 & 0.0308 & 0.0306 & 0.0016 & 0.0100 & 0.0035 & 0.0051 \\
\hline
\end{tabular}


Figure 6.1:

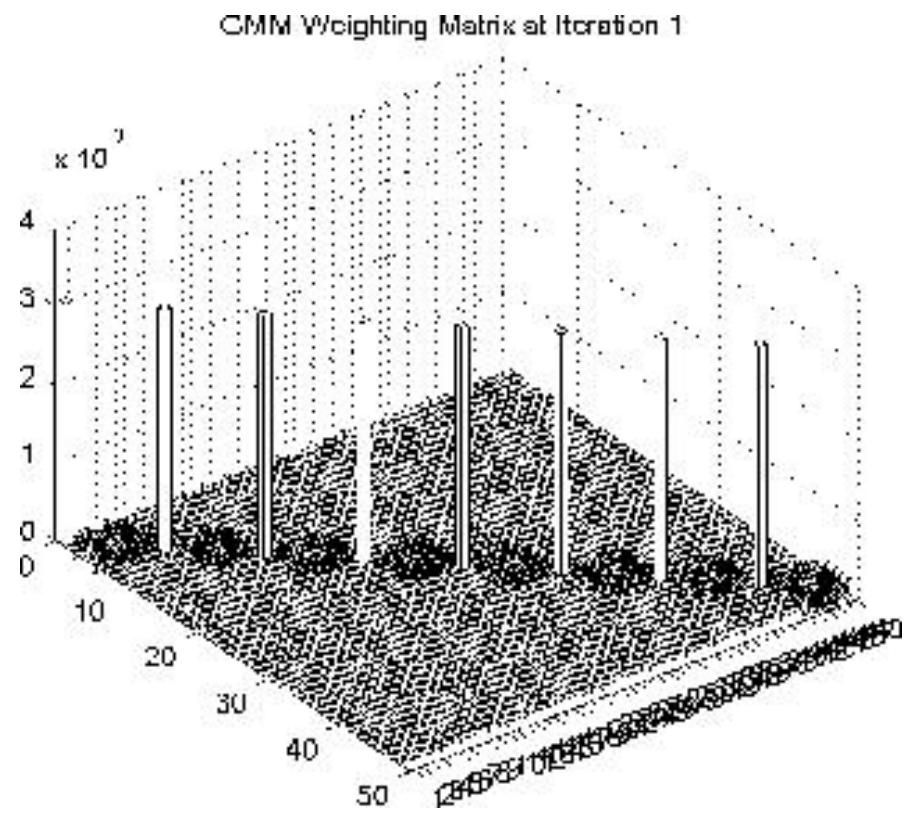

Figure 6.2:

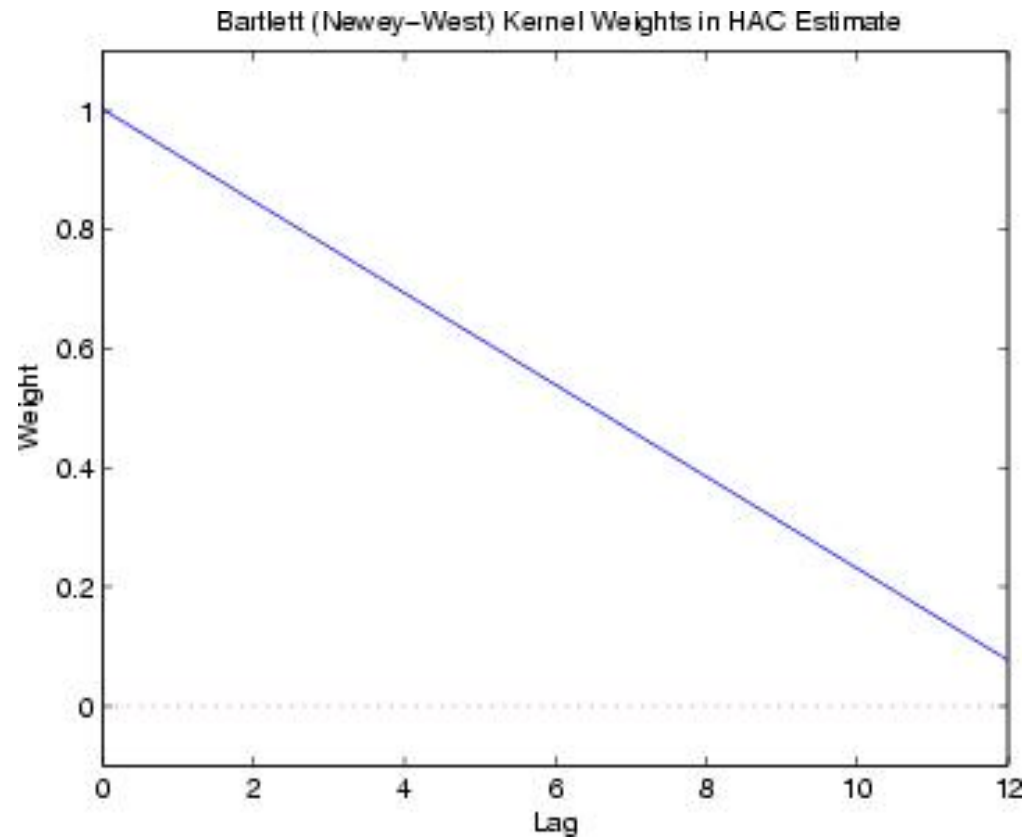


Figure 6.3:

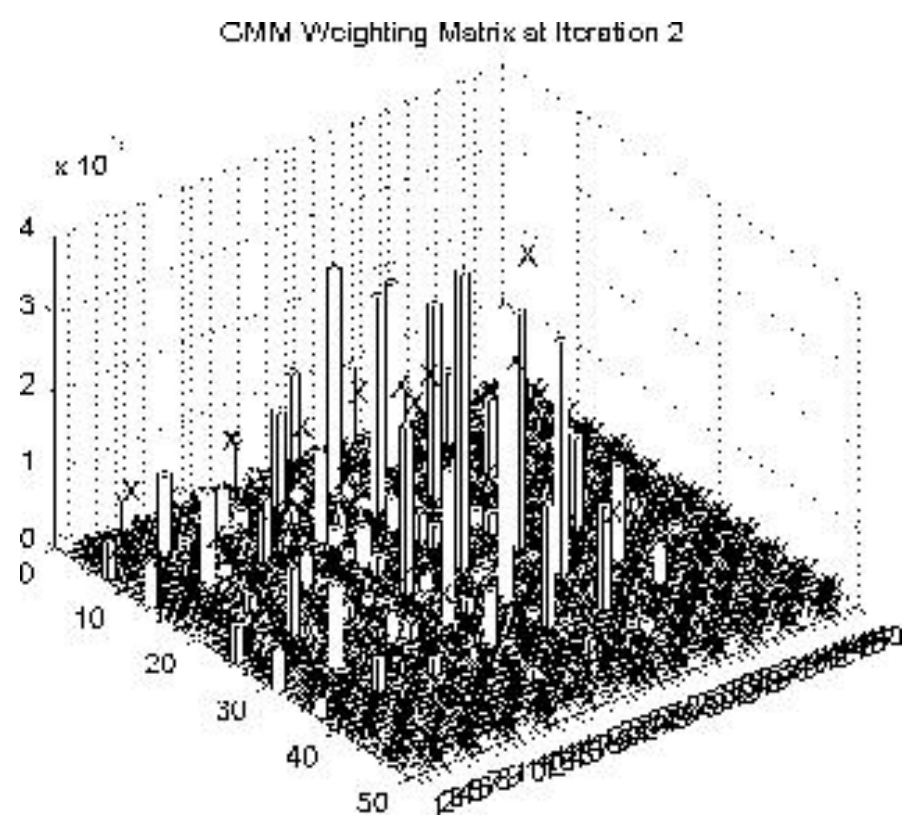




\subsection{Backward Partial Integro-Differential Equations for Option Prices}

Under the Risk-Neutral probability $Q, \mu=r$, the interest rate, and the value of a European option is defined as the discounted conditional expectation of its terminal payoff $H\left(S_{T}\right)$

$$
C_{t}=E\left[e^{-r(T-t)} H\left(S_{T}\right) \mid \mathcal{F}_{t}\right]
$$

From Markov property and set $C_{t}=C(S, t)$ we have

$$
C(S, t)=E\left[e^{-r(T-t)} H\left(S_{T}\right) \mid S_{t}=S\right] .
$$

Define $Y_{t}=r t+\beta \ln L_{t}$, hence $S_{t}=S_{0} \sigma^{2 \beta} e^{Y_{t}}$. We switch to the log space and time to maturity by letting $\tau=T-t$ and $y=\ln \left(S / S_{0}\right)-2 \beta \ln \sigma$, then

$$
e^{r \tau} C\left(S_{0} \sigma^{2 \beta} e^{y}, T-\tau\right)=E\left[H\left(S_{0} \sigma^{2 \beta} e^{y+Y_{\tau}}\right)\right]
$$

Let $c(y, \tau)=e^{r \tau} C\left(S_{0} \sigma^{2 \beta} e^{y}, T-\tau\right)$ and $g(y)=H\left(S_{0} \sigma^{2 \beta} e^{y}\right)$, then

$$
c(y, \tau)=E\left[g\left(y+Y_{\tau}\right)\right] .
$$

By the definition of infinitesimal generator of a Markov process $([3])$ ( notice here Lamperti $L_{t}$ is Markovian, so that $Y_{t}$ is also Markovian), if we denote the generator of $Y_{t}$ by $I_{Y}$, we would have

$$
I_{Y} f(x)=\lim _{t \rightarrow 0} \frac{E\left[f\left(Y_{t}+y\right)\right]-f(y)}{t} .
$$

So, if $g$ is in the domain of $I_{Y}$, and then we can differentiate with respect to $\tau$, hence obtain the integro-differential equation

$$
\frac{\partial c}{\partial \tau}=I_{Y} g
$$


on $R \times(0, T], c(y, 0)=g(y), y \in R$. Similarly, if $c$ is smooth then using a change of variable we obtain a similar equation for $C(S, t)$

$$
\frac{\partial C}{\partial t}(S, t)+I_{S}(C(S, t))(S, t)-r C(S, t)=0 \quad \text { with } C(S, T)=H(S) .
$$

This equation is similar to the Black-Scholes partial differential equation, except that the second-order differential operator is replaced by the integro-differential operator $I_{S}$.

The characterization of option prices in terms of solutions of partial integrodifferential equations allows us to use efficient numerical methods for pricing options on a single asset in presence of jumps. However, the above reasoning is heuristic: the payoff function $g$ is usually not in the domain of $I_{Y}$ and in fact it is usually not even differentiable. For example $g(y)=\left(K-S_{0} \sigma^{2 \beta} e^{y}\right)^{+}$for a put option. This lack of smoothness prevents the value function from being a classical solution of the pricing PIDE: we are led to use a notion of viscosity solution. Refer to Rama and Voltchkova 2004 ([18]) for a more detailed discussion.

Since the relation in

$$
c(y, \tau)=e^{r \tau} C\left(S_{0} \sigma^{2 \beta} e^{y}, T-\tau\right),
$$

we have

$$
\begin{gathered}
\frac{\partial c}{\partial \tau}=r C e^{r \tau}-e^{r \tau} \frac{\partial C}{\partial t}, \\
\frac{\partial c}{\partial y}=e^{r \tau} \frac{\partial C}{\partial S} S_{0} e^{y} \sigma^{2 \beta}=e^{r \tau} S \frac{\partial C}{\partial S}
\end{gathered}
$$


so we have from (6.16) that

$$
e^{r \tau} \frac{\partial C}{\partial t}(S, t)+e^{r \tau} I_{S}(C(S, t))(S, t)-r e^{r \tau} C(S, t)=0
$$

or

$$
\frac{\partial c}{\partial \tau}=e^{r \tau} I_{S}(C(S, t))(S, t)=e^{r \tau} I_{S}\left(e^{-r \tau} c(y, \tau)\right)(S, t)
$$

We use the expression of the generator $I_{S}$ in (6.8) and write

$$
\begin{gathered}
\frac{\partial c}{\partial \tau}=\left[(r-q)+a \beta e^{-y / \beta+(r-q)(T-\tau) / \beta}\right] \frac{\partial c}{\partial y} \\
+e^{\frac{-y+(r-q)(T-\tau)}{\beta}} \int_{R-\{0\}}\left[c(y+\beta \eta, \tau)-c(y, \tau)-\beta \frac{\partial c}{\partial y} h(\eta)\right] k_{\xi}(\eta) d \eta
\end{gathered}
$$

This is a partial integro-differential equation (PIDE) of $c$ in $y$ and $\tau$. It is usually referred to as the backward equation as opposed to the forward equation of $C$ in $K$ and $T$, strikes and maturities. The forward PIDE once derived is practically more attractive since it allows us, in the numerical calculation of the discretized solution, to calculate the option prices of all maturities and all strikes in relatively less calculation. In the next section we detail the forward PIDE.

\subsection{Forward Partial Integro-Differential Equations}

In this section we derive the forward PIDE for models of Markov processes in a general setup. We have a real valued Markov process $(X(t), t>0)$ with infinitesimal generator $I_{X}$ acting on functions $f$ given by

$$
I_{X}(f)=a(x, t) \frac{\partial}{\partial x} f+\frac{1}{2} b(x, t) \frac{\partial^{2}}{\partial x^{2}} f+\int_{-\infty}^{\infty}\left[f(x+\nu)-f(x)-\nu \frac{\partial}{\partial x} f\right] k(x, t, \nu) d \nu .
$$


We suppose that one may take the truncation function $h(\nu)$ to be the identity function $h(\nu)=\nu$. This merely requires that we have a special semimartingale structure for the jump and the integrability condition

$$
E\left[\int_{0}^{t} \int_{-\infty}^{\infty}\left(\nu^{2} \wedge|\nu|\right) k(X(u), u, \nu) d \nu d u\right]<\infty
$$

This condition is satisfied for a large class of models that we shall work with. In fact we shall consider locally square integrable semimartingales that satisfy the stronger condition

$$
E\left[\int_{0}^{t} \int_{-\infty}^{\infty} \nu^{2} k(X(u), u, \nu) d \nu d u\right]<\infty
$$

Let $q(t, x, T, y)$ be the transition density for the process at level $y$ at time $T>t$, given it is at level $x$ at time $t$. We are interested in first developing the forward equation for $q$ in the arguments $T, y$ and shall suppress the dependence on $t, x$. We then apply this result to European options values and develop the forward equation for option price values.

We shall also make use in our derivation of the double tail of the Lévy system defined by

$$
\tilde{\tilde{k}}(x, t, \nu)= \begin{cases}\int_{-\infty}^{\nu} \int_{-\infty}^{u} k(x, t, w) d w d u & \nu<0, \\ \int_{\nu}^{\infty} \int_{u}^{\infty} k(x, t, w) d w d u & \nu>0 .\end{cases}
$$

The double tail integrates the tail of the Lévy measure in both directions twice and hence we refer it to as the double tail. It is important as it measures quadratic variation, which may be observed by applying integration by parts two times to get

$$
\int_{-\infty}^{\infty} \tilde{\tilde{k}}(x, t, \nu) d \nu=\frac{1}{2} \int_{-\infty}^{\infty} \nu^{2} k(x, t, \nu) d \nu<\infty
$$


for locally square integrable semimartingales. In particular the double tail is itself well defined at all points of space $x$ and times $t$.

For a test function $f(y)$ we begin by defining

$$
\begin{aligned}
V_{f}(T) & =E[f(X(T)) \mid X(t)=a] \\
& =\int_{-\infty}^{\infty} q(y, T) f(y) d y .
\end{aligned}
$$

Via differentiation with respect to $T$ we get

$$
\frac{\partial}{\partial T} V_{f}(T)=\int_{-\infty}^{\infty} q_{T}(y, T) f(y) d y .
$$

In terms of the generator we have that

$$
\begin{aligned}
V_{f}(T)= & E[f(X(T)) \mid X(t)=a] \\
= & f(a)+E\left[\int_{t}^{T} I_{X}(f)(X(u), u) d u \mid X(t)=a\right] \\
= & f(a)+\int_{t}^{T} d u \int_{-\infty}^{\infty} d w q(w, u) I_{X}(f)(w, u) \\
= & f(a)+\int_{t}^{T} d u \int_{-\infty}^{\infty} d w q(w, u)\left[a(w, u) \frac{\partial}{\partial w} f+\frac{1}{2} b(w, u) \frac{\partial^{2}}{\partial w^{2}} f\right. \\
& \left.+\int_{-\infty}^{\infty}\left[f(w+\nu)-f(w)-\nu \frac{\partial}{\partial w} f\right] k(w, u, \nu) d \nu\right] .
\end{aligned}
$$

Taking the partials with respect to $T$ we obtain

$$
\begin{aligned}
\frac{\partial}{\partial T} V_{f}(T)= & \int_{-\infty}^{\infty} d w q(w, T)\left[a(w, T) \frac{\partial}{\partial w} f+\frac{1}{2} b(w, T) \frac{\partial^{2}}{\partial w^{2}} f\right. \\
& \left.+\int_{-\infty}^{\infty}\left[f(w+\nu)-f(w)-\nu \frac{\partial}{\partial w} f\right] k(w, T, \nu) d \nu\right] .
\end{aligned}
$$

We now employ integration by parts one and two times to rewrite the first two components as

$$
\int_{-\infty}^{\infty} d w q(w, T) a(w, T) \frac{\partial}{\partial w} f(w)=-\int_{-\infty}^{\infty} d y f(y) \frac{\partial}{\partial y}[q(y, T) a(y, T)],
$$




$$
\int_{-\infty}^{\infty} d w q(w, T) \frac{1}{2} b(w, T) \frac{\partial^{2}}{\partial w^{2}} f(w)=\int_{-\infty}^{\infty} d y f(y) \frac{1}{2} \frac{\partial^{2}}{\partial y^{2}}[q(y, T) b(y, T)],
$$

For the analysis of the jump integral we proceed as follows

$$
\begin{aligned}
& \int_{-\infty}^{\infty} d w q(w, T) \int_{-\infty}^{\infty}\left[f(w+\nu)-f(w)-\nu \frac{\partial}{\partial w} f\right] k(w, T, \nu) d \nu \\
= & \int_{-\infty}^{\infty} d w q(w, T) \int_{-\infty}^{\infty} \tilde{\tilde{k}}(w, T, \nu) \frac{\partial^{2}}{\partial \nu^{2}} f(w+\nu) d \nu \\
= & \int_{-\infty}^{\infty} d y \frac{\partial^{2}}{\partial y^{2}}[f(y)] \int_{-\infty}^{\infty} d \nu q(y-\nu, T) \tilde{\tilde{k}}(y-\nu, T, \nu) \\
= & \int_{-\infty}^{\infty} d y f(y) \frac{\partial^{2}}{\partial y^{2}}\left[\int_{-\infty}^{\infty} d \nu q(y-\nu, T) \tilde{\tilde{k}}(y-\nu, T, \nu)\right] .
\end{aligned}
$$

Substituting equations $(6.21,6.22,6.23)$ back into equation (6.20) we obtain

$$
\begin{array}{r}
\frac{\partial}{\partial T} V_{f}(T)=\int_{-\infty}^{\infty} d y f(y)[- \\
\frac{\partial}{\partial y}[q(y, T) a(y, T)]+\frac{1}{2} \frac{\partial^{2}}{\partial y^{2}}[q(y, T) b(y, T)] \\
\left.+\frac{\partial^{2}}{\partial y^{2}} \int_{-\infty}^{\infty} d \nu q(y-\nu, T) \tilde{\tilde{k}}(y-\nu, T, \nu)\right] .
\end{array}
$$

Comparing equations (6.19) and (6.24) for all test functions $f$ we deduce

$$
\begin{array}{r}
q_{T}(y, T)=-\frac{\partial}{\partial y}[q(y, T) a(y, T)]+\frac{1}{2} \frac{\partial^{2}}{\partial y^{2}}[q(y, T) b(y, T)] \\
+\frac{\partial^{2}}{\partial y^{2}} \int_{-\infty}^{\infty} d \nu q(y-\nu, T) \tilde{\tilde{k}}(y-\nu, T, \nu) .
\end{array}
$$

Applying this result we derive a forward equation for the prices of European options in the strike and maturity arguments. For our Markov process we take the logarithm of the stock price

$$
X(t)=\ln (S(t))
$$


For the risk neutral process we have the generator for $X$ given by

$$
\begin{aligned}
I_{X}(f)(x, u)= & \left(r-l-\frac{\sigma^{2}}{2}+\omega(x, u)\right) \frac{\partial}{\partial x} f+\frac{\sigma^{2}}{2} \frac{\partial^{2}}{\partial x^{2}} f \\
& +\int_{-\infty}^{\infty}\left[f(x+\nu)-f(x)-\nu \frac{\partial}{\partial x} f\right] k(x, u, \nu) d \nu
\end{aligned}
$$

where $r$ is the interest rate and $l$ is the dividend yield. We allow for a general space time dependent Lévy system that permits processes of infinite variation while the diffusion component is relatively simplistic and uninteresting and will in most applications be in face assumed to be null. The risk neutral drift is $r-l$. The exponential compensation of the jump component in the log price process is

$$
\omega(x, u)=-\int_{-\infty}^{\infty}\left(e^{\nu}-1-\nu\right) k(x, u, \nu) d \nu
$$

In this particular case, we may write

$$
\begin{aligned}
q_{T}(y, T)=-\frac{\partial}{\partial y}[(r-l & \left.\left.-\frac{\sigma^{2}}{2}+\omega(y, T)\right) q(y, T)\right]+\frac{\sigma^{2}}{2} \frac{\partial^{2}}{\partial y^{2}} q(y, T) \\
& +\frac{\partial^{2}}{\partial y^{2}} \int_{-\infty}^{\infty} d \nu q(y-\nu, T) \tilde{\tilde{k}}(y-\nu, T, \nu) .
\end{aligned}
$$

We now consider the price of a European call option which is given by $C(K, T)=$ $e^{-r T} \int_{\ln K}^{\infty} q(y, T)\left(e^{y}-K\right) d y$. It follows that

$$
C_{T}=-r C+e^{-r T} \int_{\ln K}^{\infty} q_{T}(y, T)\left(e^{y}-K\right) d y
$$

and

$$
\begin{array}{r}
C_{K}=-e^{-r T} \int_{\ln K}^{\infty} q(y, T) d y, \\
e^{-r T} \int_{\ln K}^{\infty} e^{y} q(y, T) d y=C-K C_{K}, \\
K C_{K K}(K, T)=e^{-r T} q(\ln K, T) .
\end{array}
$$


Substituting from equation (6.26) for $q_{T}$ we get

$$
\begin{aligned}
& C_{T}=-r C+e^{-r T} \int_{\ln K}^{\infty} d y\left(e^{y}-K\right)\left[-\frac{\partial}{\partial y}\left[\left(r-l-\frac{\sigma^{2}}{2}+\omega(y, T)\right) q(y, T)\right]+\right. \\
& \left.+\frac{\sigma^{2}}{2} \frac{\partial^{2}}{\partial y^{2}} q(y, T)+\frac{\partial^{2}}{\partial y^{2}} \int_{-\infty}^{\infty} d \nu q(y-\nu, T) \tilde{\tilde{k}}(y-\nu, T, \nu)\right] \\
& =-r C+e^{-r T} \int_{\ln K}^{\infty} d y e^{y} q(y, T)\left(r-l-\frac{\sigma^{2}}{2}+\omega(y, T)\right) \\
& +\frac{\sigma^{2}}{2} e^{-r T} \int_{\ln K}^{\infty} d y e^{y} q(y, T)+\frac{\sigma^{2}}{2} e^{-r T} K q(\ln K, T) \\
& +e^{-r T} \int_{\ln K}^{\infty} d y e^{y} \int_{-\infty}^{\infty} d \nu q(y-\nu, T) \tilde{\tilde{k}}(y-\nu, T, \nu) \\
& +e^{-r T} K \int_{-\infty}^{\infty} d \nu q(\ln K-\nu, T) \tilde{\tilde{k}}(\ln K-\nu, T, \nu) \\
& =-r C+(r-l)\left(C-K C_{K}\right)+e^{-r T} \int_{\ln K}^{\infty} d y e^{y} q(y, T) \omega(y, T)+\frac{\sigma^{2}}{2} K^{2} C_{K K} \\
& +e^{-r T} \int_{\ln K}^{\infty} d y e^{y} \int_{-\infty}^{\infty} d \nu q(y-\nu, T) \tilde{\tilde{k}}(y-\nu, T, \nu) \\
& +K^{2} \int_{-\infty}^{\infty} e^{-\nu} C_{K K}\left(K e^{-\nu}, T\right) \tilde{\tilde{k}}(\ln K-\nu, T, \nu) d \nu \\
& =-l C-(r-l) K C_{K}+\frac{\sigma^{2}}{2} K^{2} C_{K K}+e^{-r T} \int_{\ln K}^{\infty} d y e^{y} q(y, T) \omega(y, T) \\
& +K^{2} \int_{-\infty}^{\infty} e^{-\nu} C_{K K}\left(K e^{-\nu}, T\right) \tilde{\tilde{k}}(\ln K-\nu, T, \nu) d \nu \\
& +e^{-r T} \int_{\ln K}^{\infty} d y e^{y} \int_{-\infty}^{\infty} d \nu q(y-\nu, T) \tilde{\tilde{k}}(y-\nu, T, \nu) .
\end{aligned}
$$


We analyze the final two integrals as follows,

$$
\begin{aligned}
& e^{-r T} \int_{\ln K}^{\infty} d y e^{y} q(y, T) \omega(y, T) \\
& +e^{-r T} \int_{\ln K}^{\infty} d y e^{y} \int_{-\infty}^{\infty} d \nu q(y-\nu, T) \tilde{\tilde{k}}(y-\nu, T, \nu) \\
& =-e^{-r T} \int_{\ln K}^{\infty} d y e^{y} q(y, T) \int_{-\infty}^{\infty}\left(e^{\nu}-1-\nu\right) k(y, T, \nu) d \nu \\
& +e^{-r T} \int_{\ln K}^{\infty} d y e^{y} \int_{-\infty}^{\infty} d \nu q(y-\nu, T) \tilde{\tilde{k}}(y-\nu, T, \nu) \\
& =-e^{-r T} \int_{\ln K}^{\infty} d y e^{y} q(y, T) \int_{-\infty}^{\infty} e^{\nu} \tilde{\tilde{k}}(y, T, \nu) d \nu \\
& +e^{-r T} \int_{\ln K}^{\infty} d y e^{y} \int_{-\infty}^{\infty} d \nu q(y-\nu, T) \tilde{\tilde{k}}(y-\nu, T, \nu) \\
& =-e^{-r T} \int_{-\infty}^{\infty} d \nu \int_{\ln K+\nu}^{\infty} d w e^{w} q(w-\nu, T) \tilde{\tilde{k}}(w-\nu, T, \nu) \\
& +e^{-r T} \int_{-\infty}^{\infty} d \nu \int_{\ln K}^{\infty} d w e^{w} q(w-\nu, T) \tilde{\tilde{k}}(w-\nu, T, \nu) \\
& =e^{-r T} \int_{0}^{\infty} d \nu \int_{\ln K}^{\ln K+\nu} d w e^{w} q(w-\nu, T) \tilde{\tilde{k}}(w-\nu, T, \nu) \\
& -e^{-r T} \int_{-\infty}^{0} d \nu \int_{\ln K+\nu}^{\ln K} d w e^{w} q(w-\nu, T) \tilde{\tilde{k}}(w-\nu, T, \nu) \\
& =e^{-r T} \int_{\ln K}^{\infty} d w \int_{w-\ln K}^{\infty} d \nu e^{w} q(w-\nu, T) \tilde{\tilde{k}}(w-\nu, T, \nu) \\
& -e^{-r T} \int_{-\infty}^{\ln K} d w \int_{-\infty}^{w-\ln K} d w e^{w} q(w-\nu, T) \tilde{\tilde{k}}(w-\nu, T, \nu) \\
& =\int_{K}^{\infty} d U \int_{\ln \frac{U}{K}}^{\infty} d \nu e^{-r T} q(\ln U-\nu, T) \tilde{\tilde{k}}(\ln U-\nu, T, \nu) \\
& -\int_{0}^{K} d U \int_{-\infty}^{\ln \frac{U}{K}} d \nu e^{-r T} q(\ln U-\nu, T) \tilde{\tilde{k}}(\ln U-\nu, T, \nu) \\
& =\int_{K}^{\infty} d U \int_{\ln \frac{U}{K}}^{\infty} d \nu U e^{-\nu} C_{K K}\left(U e^{-\nu}, T\right) \tilde{\tilde{k}}(\ln U-\nu, T, \nu) \\
& -\int_{0}^{K} d U \int_{-\infty}^{\ln \frac{U}{K}} d \nu U e^{-\nu} C_{K K}\left(U e^{-\nu}, T\right) \tilde{\tilde{k}}(\ln U-\nu, T, \nu) .
\end{aligned}
$$

Hence we may write the final partial integral-differential equation in the call price as 


$$
\begin{aligned}
C_{T}= & -l C-(r-l) K C_{K}+\frac{\sigma^{2}}{2} K^{2} C_{K K} \\
& +K^{2} \int_{-\infty}^{\infty} e^{-\nu} C_{K K}\left(K e^{-\nu}, T\right) \tilde{\tilde{k}}(\ln K-\nu, T, \nu) d \nu \\
& +\int_{K}^{\infty} d U \int_{\ln \frac{U}{K}}^{\infty} d \nu U e^{-\nu} C_{K K}\left(U e^{-\nu}, T\right) \tilde{\tilde{k}}(\ln U-\nu, T, \nu) \\
& -\int_{0}^{K} d U \int_{-\infty}^{\ln \frac{U}{K}} d \nu U e^{-\nu} C_{K K}\left(U e^{-\nu}, T\right) \tilde{\tilde{k}}(\ln U-\nu, T, \nu),
\end{aligned}
$$

where the final three integrals may be seen as the costs of jumps to the strike, plus the costs of downcrossing and the costs of upcrossing.

The at, down and upcrossing costs are all measured by the likelihood times the level of the post jump double tail.

\subsection{Using CGMY process as the underlying Lévy}

In Section 6.1, our proposed model in (6.1), $S_{t} e^{-\mu t}$ is taken to be an exponential Lévy process which is a Martingale. We now consider CGMY pure jump process as the initial Lévy process $Z(t)$, denoted as $Z_{C G M Y}(t)$. Since introduced in [11] as a generalization of $V G$ processes, many researchers have studied the CGMY processes and have used CGMY pure jump processes in securities modeling. The Lévy triplet of CGMY process $Z(t)$ is $\left(\gamma, 0, k_{C G M Y}(x) d x\right)$, where

$$
k_{C G M Y}(x)=\frac{C e^{A x-B|x|}}{|x|^{1+Y}}
$$

is the Lévy density of $Z_{C G M Y}$ and $A=\frac{G-M}{2}, B=\frac{G+M}{2} . C>0, G \geq 0, M \geq 0$, and $Y<2$. The condition $Y<2$ is induced by the requirement that Lévy densities 
integrate $x^{2}$ in the neighborhood of 0 . The characteristic exponent is

$$
\omega(u)=i u \gamma+\int_{R-\{0\}}\left(e^{i u x}-1-i u h(x)\right) k_{C G M Y}(x) d x .
$$

The value of $\gamma$ is dependent on the choice of the truncation function $h(x)$ and particularly

$$
\gamma=\int_{R-\{0\}} h(x) k_{C G M Y}(x) d x .
$$

We know that $\exp (Z(t)-t \omega(-i))$ is a Martingale, in fact we define

$$
\omega=-\omega(-i)=-\int_{R-\{0\}}\left(e^{x}-1\right) k_{C G M Y}(x) d x,
$$

hence $\exp (Z(t)+\omega t)$ is a Martingale, which is exactly what we need.

According to (6.4), our specific Lévy process which is related to the Lamperti $L^{\xi^{(\beta)}(t)}(t)$ becomes $\xi^{(\beta)}(t)=\frac{1}{\beta}\left(Z_{C G M Y}(t)+\omega t\right)$. It is easy to see that the Lévy triplet for $\xi^{(\beta)}(t)$ is $\left(a, 0, k_{\xi}(x) d x\right)$, where $a$ and $k_{\xi}(x)$ are now related to CGMY process by

$$
a=\frac{\gamma+\omega}{\beta}=-\frac{1}{\beta} \int_{R-\{0\}}\left(e^{x}-1-h(x)\right) k_{C G M Y}(x) d x,
$$

and

$$
k_{\xi}(x) d x=\beta k_{C G M Y}(\beta x) d x .
$$

The infinitesimal generator of $\xi^{(\beta)}(t)$ is

$$
A g(\xi)=a g^{\prime}(\xi)+\int_{R-\{0\}}\left[g(\xi+\eta)-g(\xi)-g^{\prime}(\xi) h(\eta)\right] k_{\xi}(\eta) d \eta
$$

Again, we use the relation between the generators of the Lévy and the associated Lamperti to see that the generator of $L^{\left(\xi^{(\beta)}(t)\right)}(t)$ is given by

$$
B g(x)=\frac{1}{x}\left[a x g^{\prime}(x)+\int_{R-\{0\}}\left[g\left(x e^{\eta}\right)-g(x)-x g^{\prime}(x) h(\eta)\right] k_{\xi}(\eta) d \eta\right] .
$$


From (6.8), the generator of $S_{t}$ is

$$
\begin{aligned}
& I_{S}(f)(x, u) \\
= & {\left[(\mu-q)+\left(\frac{x}{S_{0} e^{(\mu-q) u} \sigma^{2 \beta}}\right)^{-1 / \beta} a \beta\right] x f^{\prime}(x) } \\
& +\left(\frac{x}{S_{0} e^{(\mu-q) u} \sigma^{2 \beta}}\right)^{-1 / \beta} \int_{R-\{0\}}\left[f\left(x e^{\beta \eta}\right)-f(x)-\beta x f^{\prime}(x) h(\eta)\right] k_{\xi}(\eta) d \eta .
\end{aligned}
$$

We may take the truncation function to be the identity function, $h(\eta)=\eta$, and by (6.28) and a simple variable change, the generator of $S_{t}$ can be written in terms of $k_{C G M Y}(x)$

$$
\begin{aligned}
& I_{S}(f)(x, u) \\
= & {\left[(\mu-q)+\left(\frac{x}{S_{0} e^{(\mu-q) u} \sigma^{2 \beta}}\right)^{-1 / \beta} a \beta\right] x f^{\prime}(x) } \\
& +\left(\frac{x}{S_{0} e^{(\mu-q) u} \sigma^{2 \beta}}\right)^{-1 / \beta} \int_{R-\{0\}}\left[f\left(x e^{\eta}\right)-f(x)-x f^{\prime}(x) \eta\right] k_{C G M Y}(\eta) d \eta .
\end{aligned}
$$

\subsubsection{Backward PIDE}

In this particular case, (6.17) is equivalent to

$$
\begin{aligned}
\frac{\partial c}{\partial \tau} & =\left[(r-q)+a \beta e^{-y / \beta+(r-q)(T-\tau) / \beta}\right] \frac{\partial c}{\partial y} \\
& +e^{\frac{-y+(r-q)(T-\tau)}{\beta}} \int_{R-\{0\}}\left[c(y+\eta, \tau)-c(y, \tau)-\frac{\partial c}{\partial y} \eta\right] k_{C G M Y}(\eta) d \eta
\end{aligned}
$$


where $a=\frac{\gamma+\omega}{\beta}$. Please refer to the Appendix about the detail evaluation of $\gamma+\omega$,

$$
\begin{aligned}
& \gamma+\omega= \\
& \left\{\begin{array}{c}
C\left[\Gamma(1-Y)\left(M^{Y-1}-G^{Y-1}\right)-\Gamma(-Y)\left[(M-1)^{Y}-M^{Y}+(G+1)^{Y}-G^{Y}\right]\right] \\
Y<0 \\
C \Gamma(1-Y)\left[M^{Y-1}-G^{Y-1}+\frac{(M-1)^{Y}}{Y}-\frac{M^{Y}}{Y}+\frac{(G+1)^{Y}}{Y}-\frac{G^{Y}}{Y}\right] \\
0<Y<1 ; \\
C \frac{\Gamma(2-Y)}{Y(1-Y)}\left[(M-1)^{Y}-M^{Y}+(G+1)^{Y}-G^{Y}+Y M^{Y-1}-Y G^{Y-1}\right] \\
1<Y<2 .
\end{array}\right.
\end{aligned}
$$

\subsubsection{Forward PIDE}

We derive the Forward PIDE in order to more efficiently calculate the option prices of all maturities and all strikes on a fixed asset. We follow the general derivation of Section 6.5

In the Risk-Neutral world $\mu=r$, the interest rate. For our Markov process we take the logarithm of the stock price $X(t)=\ln S(t)$. Since the infinitesimal generator of $S(t)$ has been derived earlier to be

$$
\begin{aligned}
I_{S}(f)(x, u)= & {\left[(r-l) x+a \beta x\left(\frac{x}{S_{0} e^{(r-l) u} \sigma^{2 \beta}}\right)^{-\frac{1}{\beta}}\right] \frac{\partial}{\partial x} f } \\
& +\left(\frac{x}{S_{0} e^{(r-l) u} \sigma^{2 \beta}}\right)^{-\frac{1}{\beta}} \int_{R-\{0\}}\left[f\left(x e^{\eta}\right)-f(x)-x \eta \frac{\partial}{\partial x} f\right] k_{C G M Y}(\eta) d \eta,
\end{aligned}
$$

where the truncation function has been taken to be identity function, and $l$ denotes the dividend yield to avoid confusion with density $q$. 
It is easy to derive the generator for $X(t)$

$$
I_{X}(g)(x, u)=a(x, u) \frac{\partial}{\partial x} g+\int_{R-\{0\}}\left[g(x+\eta)-g(x)-\eta \frac{\partial}{\partial x} g\right] k(x, u, \eta) d \eta
$$

where

$$
\begin{aligned}
& a(x, u)=(r-l)+a \beta\left(\frac{e^{x}}{S_{0} e^{(r-l) u} \sigma^{2 \beta}}\right)^{-\frac{1}{\beta}}, \\
& k(x, u, \nu)=\left(\frac{e^{x}}{S_{0} e^{(r-l) u} \sigma^{2 \beta}}\right)^{-\frac{1}{\beta}} k_{C G M Y}(\nu) .
\end{aligned}
$$

Recall that $a=\frac{\gamma+\omega}{\beta}$, and $\gamma+\omega=-\int_{R-\{0\}}\left(e^{\nu}-1-\nu\right) k_{C G M Y}(\nu) d \nu$, so now

$$
a(x, u)=(r-l)+w(x, u),
$$

where

$$
w(x, u)=-\int_{R-\{0\}}\left(e^{\nu}-1-\nu\right) k(x, u, \nu) d \nu
$$

Here, we have $b(x, u)=0$, which implies no diffusion part. Hence by the result in ( 6.26), we have

$$
\begin{gathered}
q_{T}(y, T)=-\frac{\partial}{\partial y}[(r-l+w(y, T)) q(y, T)] \\
+\frac{\partial^{2}}{\partial y^{2}} \int_{R-\{0\}} \tilde{\tilde{k}}(y-\nu, T, \nu) q(y-\nu, T) d \nu .
\end{gathered}
$$

For European Call option, we know that

$$
C(K, T)=e^{-r T} \int_{\ln K}^{\infty} q(y, T)\left(e^{y}-K\right) d y
$$


Again $q(t, x, T, y)$ denote the transition density for the process $X(t)$ to be at level $y$ at time $T>t$ give it is at level $x$ at time $t$. We keep the dependence of $q$ on $y$ and $T$, and suppress the dependence on $x$ and $t$.

It is easy to see that

$$
\begin{array}{r}
C_{K}=-e^{-r T} \int_{\ln K}^{\infty} q(y, T) d y, \\
e^{-r T} \int_{\ln K}^{\infty} e^{y} q(y, T) d y=C-K C_{K}, \\
K C_{K K}(K, T)=e^{-r T} q(\ln K, T),
\end{array}
$$

and

$$
C_{T}=-r C+e^{-r T} \int_{\ln K}^{\infty} q_{T}(y, T)\left(e^{y}-K\right) d y
$$

Putting $q_{T}(y, T)$ into $(6.30)$, we obtain an equation analogous to what we have in (6.27) except that the diffusion part is no longer present

$$
\begin{aligned}
C_{T}= & -l C-(r-l) K C_{K} & \\
& +K^{2} \int_{R-\{0\}} e^{-\nu} C_{K K}\left(K e^{-\nu}, T\right) \tilde{\tilde{k}}(\ln K-\nu, T, \nu) d \nu & \text { part1 } \\
& +\int_{K}^{\infty} d U \int_{\ln \left(\frac{U}{K}\right)}^{\infty} d \nu U e^{-\nu} C_{K K}\left(U e^{-\nu}, T\right) \tilde{\tilde{k}}(\ln U-\nu, T, \nu) & \text { part2 } \\
& -\int_{0}^{K} d U \int_{-\infty}^{\ln \left(\frac{U}{K}\right)} d \nu U e^{-\nu} C_{K K}\left(U e^{-\nu}, T\right) \tilde{\tilde{k}}(\ln U-\nu, T, \nu) . & \text { part3 }
\end{aligned}
$$

We now introduce the density function of CGMY process into the system, we obtain from (6.29)

$$
k(x, u, \nu)=\left(\frac{e^{x}}{S_{0} e^{(r-l) u} \sigma^{2 \beta}}\right)^{-\frac{1}{\beta}} \frac{C e^{A \nu-B|\nu|}}{|\nu|^{1+Y}},
$$


where $A=\frac{G-M}{2}, B=\frac{G+M}{2}$, and $C>0, G, M \geq 0$ and $Y<2$.

By setting

$$
D=\left(S_{0} \sigma^{2 \beta}\right)^{\frac{1}{\beta}}
$$

then,

$$
k(x, u, \nu)=C D e^{-\frac{x}{\beta}} e^{\frac{r-l}{\beta} u+A \nu-B|\nu|}|\nu|^{-(1+Y)},
$$

Recall that the double tail $\tilde{\tilde{k}}$ is defined by

$$
\tilde{\tilde{k}}(x, t, \nu)=\left\{\begin{array}{cc}
\int_{-\infty}^{\nu} \int_{-\infty}^{u} k(x, t, w) d w d u & \nu<0 \\
\int_{\nu}^{\infty} \int_{u}^{\infty} k(x, t, w) d w d u & \nu>0
\end{array}\right.
$$

We now take care of the three integral parts one by one.

$$
\begin{aligned}
\operatorname{part1}= & K^{2} \int_{R-\{0\}} e^{-\nu} C_{K K}\left(K e^{-\nu}, T\right) \tilde{\tilde{k}}(\ln K-\nu, T, \nu) d \nu \\
= & K^{2} \int_{-\infty}^{0} d \nu e^{-\nu} C_{K K}\left(K e^{-\nu}, T\right)\left(\int_{-\infty}^{\nu} \int_{-\infty}^{u} k(\ln K-\nu, T, w) d w d u\right) \\
& +K^{2} \int_{0}^{\infty} d \nu e^{-\nu} C_{K K}\left(K e^{-\nu}, T\right)\left(\int_{\nu}^{\infty} \int_{u}^{\infty} k(\ln K-\nu, T, w) d w d u\right) \\
= & K^{2} C D e^{\frac{r-l}{\beta} T} \int_{-\infty}^{0} e^{-\nu} C_{K K}\left(K e^{-\nu}, T\right)\left(e^{-\nu} K\right)^{-\frac{1}{\beta}} f_{1}(\nu), d \nu \\
& +K^{2} C D e^{\frac{r-l}{\beta} T} \int_{0}^{\infty} e^{-\nu} C_{K K}\left(K e^{-\nu}, T\right)\left(e^{-\nu} K\right)^{-\frac{1}{\beta}} f_{2}(\nu) d \nu,
\end{aligned}
$$

where

$$
\begin{aligned}
f_{1}(\nu) & =\int_{-\infty}^{\nu} \int_{-\infty}^{u} e^{G w}(-w)^{-(1+Y)} d w d u \\
f_{2}(\nu) & =\int_{\nu}^{\infty} \int_{u}^{\infty} e^{-M w}(w)^{-(1+Y)} d w d u
\end{aligned}
$$


after change of variable by setting $U=e^{-\nu} K$, we have then

$$
\begin{aligned}
\text { part } 1= & K C D e^{\frac{r-l}{\beta} T} \int_{K}^{\infty} C_{K K}(U, T) U^{-\frac{1}{\beta}} f_{1}\left(\ln \frac{K}{U}\right) d U \\
& +K C D e^{\frac{r-l}{\beta} T} \int_{0}^{K} C_{K K}(U, T) U^{-\frac{1}{\beta}} f_{2}\left(\ln \frac{K}{U}\right) d U .
\end{aligned}
$$

Now let us take care of part2 and part3 together,

$$
\begin{array}{ccc} 
& \text { part2 }+ \text { part3 } \\
= & \int_{K}^{\infty} d U \int_{\ln \left(\frac{U}{K}\right)}^{\infty} d \nu U e^{-\nu} C_{K K}\left(U e^{-\nu}, T\right) \tilde{\tilde{k}}(\ln U-\nu, T, \nu) \\
& -\int_{0}^{K} d U \int_{-\infty}^{\ln \left(\frac{U}{K}\right)} d \nu U e^{-\nu} C_{K K}\left(U e^{-\nu}, T\right) \tilde{\tilde{k}}(\ln U-\nu, T, \nu) \\
=\quad & C D e^{\frac{r-l}{\beta} T} \int_{K}^{\infty} d U \int_{\ln \left(\frac{U}{K}\right)}^{\infty} d \nu\left(U e^{-\nu} C_{K K}\left(U e^{-\nu}, T\right)\left(e^{-\nu} U\right)^{-\frac{1}{\beta}} f_{2}(\nu)\right) \\
& -C D e^{\frac{r-l}{\beta} T} \int_{0}^{K} d U \int_{-\infty}^{\ln \left(\frac{U}{K}\right)} d \nu\left(U e^{-\nu} C_{K K}\left(U e^{-\nu}, T\right)\left(e^{-\nu} U\right)^{-\frac{1}{\beta}} f_{1}(\nu)\right) \\
& C D e^{\frac{r-l}{\beta} T} \int_{K}^{\infty} d U \int_{0}^{K} C_{K K}(W, T) W^{-\frac{1}{\beta}} f_{2}\left(\ln \frac{U}{W}\right) d W \\
& -C D e^{\frac{r-l}{\beta} T} \int_{0}^{K} d U \int_{K}^{\infty} C_{K K}(W, T) W^{-\frac{1}{\beta}} f_{1}\left(\ln \frac{U}{W}\right) d W .
\end{array}
$$

Finally, our forward PIDE in $K$ and $T$ is

$$
\begin{aligned}
& C_{T}=-l C-(r-l) K C_{K} \\
& +K C D e^{\frac{r-l}{\beta} T} \int_{K}^{\infty} C_{K K}(U, T) U^{-\frac{1}{\beta}} f_{1}\left(\ln \frac{K}{U}\right) d U \\
& +K C D e^{\frac{r-l}{\beta} T} \int_{0}^{K} C_{K K}(U, T) U^{-\frac{1}{\beta}} f_{2}\left(\ln \frac{K}{U}\right) d U \quad \text { part1 } \\
& +C D e^{\frac{r-l}{\beta} T} \int_{K}^{\infty} d U \int_{0}^{K} C_{K K}(W, T) W^{-\frac{1}{\beta}} f_{2}\left(\ln \frac{U}{W}\right) d W \quad \text { part2 } \\
& -C D e^{\frac{r-l}{\beta} T} \int_{0}^{K} d U \int_{K}^{\infty} C_{K K}(W, T) W^{-\frac{1}{\beta}} f_{1}\left(\ln \frac{U}{W}\right) d W . \quad \text { part3 }
\end{aligned}
$$

We switch to the log strike space by defining 


$$
k=\ln K \text {. }
$$

This transform will benefit the discretization of the system. Strike prices are often times sparsely distributed in a large range, which brings difficulty to the discretization of the system when we always require the spacing of $K$ be small enough to achieve convergence. Because of the large range of strike prices, small spacing will lead to too many points which will substantially slow down the calculation, although most of these points are not even traded strikes of our interest. By the transform from strike space to log strike space, we achieve small spacing without making the system huge. We define

$$
c(k, T)=C\left(e^{k}, T\right)=C(K, T),
$$

so that

$$
K C_{K}(K, T)=c_{k}(k, T), \quad C_{K K}(K, T)=e^{-2 k}\left[c_{k k}(k, T)-c_{k}(k, T)\right]
$$


then, the forward PIDE becomes

$$
\begin{aligned}
& c_{T} \\
= & -l c(k, T)-(r-l) c_{k}(k, T) \\
+ & K C D e^{\frac{r-l}{\beta} T}\left[\int_{K}^{\infty}\left[c_{k k}(\ln U, T)-c_{k}(\ln U, T)\right] U^{-\frac{1}{\beta}-2} f_{1}\left(\ln \frac{K}{U}\right) d U\right. \\
& \left.+\int_{0}^{K}\left[c_{k k}(\ln U, T)-c_{k}(\ln U, T)\right] U^{-\frac{1}{\beta}-2} f_{2}\left(\ln \frac{K}{U}\right) d U\right] \quad \text { part } 1 \\
+ & C D e^{\frac{r-l}{\beta} T} \int_{K}^{\infty} d U \int_{0}^{K}\left[c_{k k}(\ln W, T)-c_{k}(\ln W, T)\right] W^{-\frac{1}{\beta}-2} f_{2}\left(\ln \frac{U}{W}\right) d W \text { part2 } \\
- & C D e^{\frac{r-l}{\beta} T} \int_{0}^{K} d U \int_{K}^{\infty}\left[c_{k k}(\ln W, T)-c_{k}(\ln W, T)\right] W^{-\frac{1}{\beta}-2} f_{1}\left(\ln \frac{U}{W}\right) d W . \text { part3 }
\end{aligned}
$$

\subsubsection{Discretization}

We discretize this system with $N+1$ mesh points in $k$-direction and $M+1$ mesh points in $T$-direction. So:

$$
\Delta k=\frac{k_{\max }-k_{\min }}{N}, \quad \Delta T=\frac{T_{\max }-T_{\min }}{M} .
$$

Hence $T_{1}=T_{\min }, T_{M+1}=T_{\max }, T_{j}=T_{1}+(j-1) \Delta T$ for all $j=1, \ldots, M+1$, and $k_{1}=k_{\text {min }}=\ln K_{\text {min }}, k_{N+1}=k_{\max }=\ln K_{\text {max }}, k_{i}=k_{1}+(i-1) \Delta k$ for all $i=1, \ldots, N+1$. We notice that the values in $k$ and values in $K$ (from real data) do not necessarily form a one to one correspondance, but we can always recover the prices at desired strike prices by performing numerical interpolation.

Using

$$
c_{i, j} \approx c\left(k_{i}, T_{j}\right)
$$

we approximate the derivatives by: 


$$
\begin{gathered}
c_{T}\left(k_{i}, T_{j}\right) \approx \frac{c_{i, j+1}-c_{i, j}}{\Delta T}, \\
c_{k}\left(k_{i}, T_{j}\right) \approx \frac{c_{i+1, j}-c_{i-1, j}}{2 \Delta k}, \\
c_{k k}\left(k_{i}, T_{j}\right) \approx \frac{c_{i+1, j}+c_{i-1, j}-2 c_{i, j}}{(\Delta k)^{2}} .
\end{gathered}
$$

Initial condition

$$
c\left(k_{i}, T_{1}\right)=\left[S_{T_{1}}-e^{k_{i}}\right]^{+} \text {for all } i=1, \ldots, N+1 .
$$

Boundary conditions

$$
\begin{gathered}
c\left(k_{1}, T_{j}\right)=S_{T_{1}} e^{-l T_{j}}-e^{k_{1}} e^{-r T_{j}} \text { for all } j=1, \ldots, M+1, \\
c\left(k_{N+1}, T_{j}\right)=0 \text { for all } j=1, \ldots, M+1 .
\end{gathered}
$$

Using these notations, now we discretize equation (6.32). Here we adopt a mixed approach. For the jump terms, we use an explicit approach so that the matrix to be inverted at each time step is tri-diagonal. On the rest of the PIDE, a fully implicit approach is used.

$$
\frac{c_{i, j+1}-c_{i, j}}{\Delta T}=-l c_{i, j+1}-(r-l) \frac{c_{i+1, j+1}-c_{i-1, j+1}}{2 \Delta k}+\operatorname{part} 1+\text { part } 2+\text { part } 3,
$$

or setting $\lambda=\frac{\Delta T}{2 \Delta k}$, we have

$$
\begin{array}{r}
c_{i, j+1}-c_{i, j}=-l c_{i, j+1} \Delta T-(r-l) \lambda\left(c_{i+1, j+1}-c_{i-1, j+1}\right) \\
+(\operatorname{part} 1+\text { part } 2+\text { part } 3) \Delta T .
\end{array}
$$


The discretization of part1 and part2+part3 are taken care of as following, denoting $K_{i}=e^{k_{i}}$.

part 1

$$
\begin{aligned}
& =K_{i} C D e^{\frac{r-l}{\beta} T_{j}}\left[\int_{K_{i}}^{\infty}\left[c_{k k}\left(\ln U, T_{j}\right)-c_{k}\left(\ln U, T_{j}\right)\right] U^{-\frac{1}{\beta}-2} f_{1}\left(\ln \frac{K_{i}}{U}\right) d U\right. \\
& \left.+\int_{0}^{K_{i}}\left[c_{k k}\left(\ln U, T_{j}\right)-c_{k}\left(\ln U, T_{j}\right)\right] U^{-\frac{1}{\beta}-2} f_{2}\left(\ln \frac{K_{i}}{U}\right) d U\right] \\
& =K_{i} C D e^{\frac{r-l}{\beta} T_{j}}\left[\int_{K_{i}}^{K_{N+1}}\left[c_{k k}\left(\ln U, T_{j}\right)-c_{k}\left(\ln U, T_{j}\right)\right] U^{-\frac{1}{\beta}-2} f_{1}\left(\ln \frac{K_{i}}{U}\right) d U\right. \\
& \left.+\int_{K_{1}}^{K_{i}}\left[c_{k k}\left(\ln U, T_{j}\right)-c_{k}\left(\ln U, T_{j}\right)\right] U^{-\frac{1}{\beta}-2} f_{2}\left(\ln \frac{K_{i}}{U}\right) d U\right] \\
& =K_{i} C D e^{\frac{r-l}{\beta} T_{j}}\left[\sum_{m=i+1}^{N} \int_{K_{m}}^{K_{m+1}}\left[c_{k k}\left(\ln U, T_{j}\right)-c_{k}\left(\ln U, T_{j}\right)\right] U^{-\frac{1}{\beta}-2} f_{1}\left(\ln \frac{K_{i}}{U}\right) d U\right. \\
& \left.+\sum_{m=2}^{i-1} \int_{K_{m-1}}^{K_{m}}\left[c_{k k}\left(\ln U, T_{j}\right)-c_{k}\left(\ln U, T_{j}\right)\right] U^{-\frac{1}{\beta}-2} f_{2}\left(\ln \frac{K_{i}}{U}\right) d U\right] \\
& =K_{i} C D e^{\frac{r-l}{\beta} T_{j}}[ \\
& \sum_{m=i+1}^{N} \int_{K_{m}}^{K_{m+1}}\left[\frac{c_{m+1, j}+c_{m-1, j}-2 c_{m, j}}{(\Delta k)^{2}}-\frac{c_{m+1, j}-c_{m-1, j}}{2 \Delta k}\right] U^{-\frac{1}{\beta}-2} f_{1}\left(\ln \frac{K_{i}}{U}\right) d U \\
& \left.+\sum_{m=2}^{i-1} \int_{K_{m-1}}^{K_{m}}\left[\frac{c_{m+1, j}+c_{m-1, j}-2 c_{m, j}}{(\Delta k)^{2}}-\frac{c_{m+1, j}-c_{m-1, j}}{2 \Delta k}\right] U^{-\frac{1}{\beta}-2} f_{2}\left(\ln \frac{K_{i}}{U}\right) d U\right] \\
& =\frac{K_{i} C D e^{\frac{r-l}{\beta} T_{j}}}{2(\Delta k)^{2}}[ \\
& \sum_{m=i+1}^{N}\left[(2-\Delta k) c_{m+1, j}-4 c_{m, j}+(2+\Delta k) c_{m-1, j}\right] \int_{K_{m}}^{K_{m+1}} U^{-\frac{1}{\beta}-2} f_{1}\left(\ln \frac{K_{i}}{U}\right) d U \\
& \left.+\sum_{m=2}^{i-1}\left[(2-\Delta k) c_{m+1, j}-4 c_{m, j}+(2+\Delta k) c_{m-1, j}\right] \int_{K_{m-1}}^{K_{m}} U^{-\frac{1}{\beta}-2} f_{2}\left(\ln \frac{K_{i}}{U}\right) d U\right] .
\end{aligned}
$$


Similarly,

$$
\begin{aligned}
& \text { part } 2+\text { part } 3 \\
& =C D e^{\frac{r-l}{\beta} T_{j}}\left[\int_{K_{i}}^{\infty} d U \int_{0}^{K_{i}}\left[c_{k k}\left(\ln W, T_{j}\right)-c_{k}\left(\ln W, T_{j}\right)\right] W^{-\frac{1}{\beta}-2} f_{2}\left(\ln \frac{U}{W}\right) d W\right. \\
& \left.-\int_{0}^{K_{i}} d U \int_{K_{i}}^{\infty}\left[c_{k k}\left(\ln W, T_{j}\right)-c_{k}\left(\ln W, T_{j}\right)\right] W^{-\frac{1}{\beta}-2} f_{1}\left(\ln \frac{U}{W}\right) d W\right] \\
& =C D e^{\frac{r-l}{\beta} T_{j}}\left[\int_{K_{i}}^{K_{N+1}} d U \int_{K_{1}}^{K_{i}}\left[c_{k k}\left(\ln W, T_{j}\right)-c_{k}\left(\ln W, T_{j}\right)\right] W^{-\frac{1}{\beta}-2} f_{2}\left(\ln \frac{U}{W}\right) d W\right. \\
& \left.-\int_{K_{1}}^{K_{i}} d U \int_{K_{i}}^{K_{N+1}}\left[c_{k k}\left(\ln W, T_{j}\right)-c_{k}\left(\ln W, T_{j}\right)\right] W^{-\frac{1}{\beta}-2} f_{1}\left(\ln \frac{U}{W}\right) d W\right] \\
& =C D e^{\frac{r-l}{\beta} T_{j}}\left[\int _ { K _ { i } } ^ { K _ { N + 1 } } d U \sum _ { m = 2 } ^ { i - 1 } \int _ { K _ { m - 1 } } ^ { K _ { m } } \left(\frac{c_{m+1, j}+c_{m-1, j}-2 c_{m, j}}{(\Delta k)^{2}}\right.\right. \\
& \left.-\frac{c_{m+1, j}-c_{m-1, j}}{2 \Delta k}\right) W^{-\frac{1}{\beta}-2} f_{2}\left(\ln \frac{U}{W}\right) d W \\
& -\int_{K_{1}}^{K_{i}} d U \sum_{m=i+1}^{N} \int_{K_{m}}^{K_{m+1}}\left(\frac{c_{m+1, j}+c_{m-1, j}-2 c_{m, j}}{(\Delta k)^{2}}\right. \\
& \left.\left.-\frac{c_{m+1, j}-c_{m-1, j}}{2 \Delta k}\right) W^{-\frac{1}{\beta}-2} f_{1}\left(\ln \frac{U}{W}\right) d W\right] \\
& =\frac{C D e^{\frac{r-l}{\beta} T_{j}}}{2(\Delta k)^{2}}\left[\sum _ { m = 2 } ^ { i - 1 } \left[(2-\Delta k) c_{m+1, j}-4 c_{m, j}\right.\right. \\
& \left.+(2+\Delta k) c_{m-1, j}\right] \int_{K_{i}}^{K_{N+1}} d U \int_{K_{m-1}}^{K_{m}} W^{-\frac{1}{\beta}-2} f_{2}\left(\ln \frac{U}{W}\right) d W \\
& -\sum_{m=i+1}^{N}\left[(2-\Delta k) c_{m+1, j}-4 c_{m, j}\right. \\
& \left.\left.+(2+\Delta k) c_{m-1, j}\right] \int_{K_{1}}^{K_{i}} d U \int_{K_{m}}^{K_{m+1}} W^{-\frac{1}{\beta}-2} f_{1}\left(\ln \frac{U}{W}\right) d W\right] .
\end{aligned}
$$

Rearranging (6.33), we have

$$
\begin{array}{r}
(l-r) \lambda c_{i-1, j+1}+(1+l \Delta T) c_{i, j+1}+(r-l) \lambda c_{i+1, j+1} \\
=c_{i, j}+(\text { part } 1+\operatorname{part} 2+\operatorname{part} 3) \Delta T .
\end{array}
$$

In fact, putting similar summations together in part1, part2 and part3, we eventually 
obtain the discretized system

$$
\begin{array}{r}
(l-r) \lambda c_{i-1, j+1}+(1+l \Delta T) c_{i, j+1}+(r-l) \lambda c_{i+1, j+1} \\
=c_{i, j}+\frac{\Delta T C D e^{\frac{r-l}{\beta} T_{j}}}{2(\Delta k)^{2}}(I+I I),
\end{array}
$$

where

$$
\begin{aligned}
I= & \sum_{m=2}^{i-1}\left[(2-\Delta k) c_{m+1, j}-4 c_{m, j}+(2+\Delta k) c_{m-1, j}\right][ \\
& K_{i} \int_{K_{m-1}}^{K_{m}} U^{-\frac{1}{\beta}-2} f_{2}\left(\ln \frac{K_{i}}{U}\right) d U \\
& \left.+\int_{K_{i}}^{K_{N+1}} d U \int_{K_{m-1}}^{K_{m}} W^{-\frac{1}{\beta}-2} f_{2}\left(\ln \frac{U}{W}\right) d W\right]
\end{aligned}
$$

and

$$
\begin{aligned}
I I= & \sum_{m=i+1}^{N}\left[(2-\Delta k) c_{m+1, j}-4 c_{m, j}+(2+\Delta k) c_{m-1, j}\right][ \\
& K_{i} \int_{K_{m}}^{K_{m+1}} U^{-\frac{1}{\beta}-2} f_{1}\left(\ln \frac{K_{i}}{U}\right) d U \\
& \left.-\int_{K_{1}}^{K_{i}} d U \int_{K_{m}}^{K_{m+1}} W^{-\frac{1}{\beta}-2} f_{1}\left(\ln \frac{U}{W}\right) d W\right]
\end{aligned}
$$

We see that at each time step, we will solve a tridiagonal matrix system.

In the calculation of I and II, we would come to the calculation of four particular integrals, which are

$$
\begin{array}{r}
\int_{K_{m}}^{K_{m+1}} U^{-\frac{1}{\beta}-2} f_{1}\left(\ln \frac{K_{i}}{U}\right) d U, \\
\int_{K_{m-1}}^{K_{m}} U^{-\frac{1}{\beta}-2} f_{2}\left(\ln \frac{K_{i}}{U}\right) d U, \\
\int_{K_{i}}^{K_{N+1}} d U \int_{K_{m-1}}^{K_{m}} W^{-\frac{1}{\beta}-2} f_{2}\left(\ln \frac{U}{W}\right) d W, \\
\int_{K_{1}}^{K_{i}} d U \int_{K_{m}}^{K_{m+1}} W^{-\frac{1}{\beta}-2} f_{1}\left(\ln \frac{U}{W}\right) d W,
\end{array}
$$


where recall that

$$
\begin{array}{cc}
f_{1}(\nu)=\int_{-\infty}^{\nu} \int_{-\infty}^{u} e^{G w}(-w)^{-(1+Y)} d w d u & \text { for } \nu<0, \\
f_{2}(\nu)=\int_{\nu}^{\infty} \int_{u}^{\infty} e^{-M w}(w)^{-(1+Y)} d w d u & \text { for } \nu>0 .
\end{array}
$$

To simplify, we set $\hat{\beta}=\frac{\beta}{1+2 \beta}$. Hence, we need to calculate the integrals

$$
\begin{array}{r}
\int_{K_{m}}^{K_{m+1}} U^{-\frac{1}{\beta}} f_{1}\left(\ln \frac{K_{i}}{U}\right) d U, \\
\int_{K_{m-1}}^{K_{m}} U^{-\frac{1}{\beta}} f_{2}\left(\ln \frac{K_{i}}{U}\right) d U, \\
\int_{K_{i}}^{K_{N+1}} d U \int_{K_{m-1}}^{K_{m}} W^{-\frac{1}{\beta}} f_{2}\left(\ln \frac{U}{W}\right) d W, \\
\int_{K_{1}}^{K_{i}} d U \int_{K_{m}}^{K_{m+1}} W^{-\frac{1}{\beta}} f_{1}\left(\ln \frac{U}{W}\right) d W .
\end{array}
$$

The evaluation of these integrals is included in the Appendix B considering the lengthy integral manipulation. Section 6.6.4 contains the evaluation of $f_{1}$ and $f_{2}$ employing the numerical Fast Fourier Transformation. Section 6.6.5 concludes the discretized system by putting all the results together.

\subsubsection{The Evaluation of $f_{1}(a)$ and $f_{2}(a)$}

We define

$$
f(\nu)=I_{\{\nu<0\}} f_{1}(\nu)+I_{\{\nu>0\}} f_{2}(\nu),
$$

where

$$
\begin{aligned}
& f_{1}=\int_{-\infty}^{\nu} \int_{-\infty}^{u} e^{G w}(-w)^{-(1+Y)} d w d u, \\
& f_{2}=\int_{\nu}^{\infty} \int_{u}^{\infty} e^{-M w} w^{-(1+Y)} d w d u .
\end{aligned}
$$


We can see that function $f(\nu)$ is actually the double tail which was introduced in section (6.5) of the CGMY density. It is well defined and square integrable.

The Fourier Transform of $f(\nu)$ is:

$$
\begin{aligned}
& \hat{f}(\xi)=\int_{-\infty}^{\infty} e^{i \xi \nu} f(\nu) d \nu \\
& =\int_{-\infty}^{0} e^{i \xi \nu} d \nu\left(\int_{-\infty}^{\nu} \int_{-\infty}^{u} e^{G w}(-w)^{-(1+Y)} d w d u\right) \\
& +\int_{0}^{\infty} e^{i \xi \nu} d \nu\left(\int_{\nu}^{\infty} \int_{u}^{\infty} e^{-M w} w^{-(1+Y)} d w d u\right) \\
& =\frac{1}{i \xi}\left[\int_{-\infty}^{0} \int_{-\infty}^{u} e^{G w}(-w)^{-(1+Y)} d w d u-\int_{-\infty}^{0} e^{i \xi \nu} \int_{-\infty}^{\nu} e^{G w}(-w)^{-(1+Y)} d w d \nu\right] \\
& -\frac{1}{i \xi} \int_{0}^{\infty} \int_{u}^{\infty} e^{-M w} w^{-(1+Y)} d w d u+\frac{1}{i \xi} \int_{0}^{\infty} e^{i \xi \nu} \int_{\nu}^{\infty} e^{-M w} w^{-(1+Y)} d w d \nu \\
& =\frac{1}{i \xi}\left[-\int_{-\infty}^{0} u e^{G u}(-u)^{-(1+Y)} d u-\frac{1}{i \xi} \int_{-\infty}^{0} e^{G w}(-w)^{-(1+Y)} d w\right. \\
& \left.+\frac{1}{i \xi} \int_{-\infty}^{0} e^{i \xi+G}(-\nu)^{-(1+Y)} d \nu\right]-\frac{1}{i \xi} \int_{0}^{\infty} u e^{-M u} u^{-(1+Y)} d u \\
& -\frac{1}{\xi^{2}}\left(-\int_{0}^{\infty} e^{-M w} w^{-(1+Y)} d w+\int_{0}^{\infty} e^{i \xi \nu} e^{-M \nu} \nu^{-(1+Y)} d \nu\right) \\
& =\frac{1}{i \xi} \int_{-\infty}^{0} e^{G u}(-u)^{-Y} d u+\frac{1}{\xi^{2}} \int_{-\infty}^{0} e^{G w}(-w)^{-(1+Y)} d w \\
& -\frac{1}{\xi^{2}} \int_{-\infty}^{0} e^{(G+i \xi) \nu}(-\nu)^{-(1+Y)} d \nu-\frac{1}{i \xi} \int_{0}^{\infty} e^{-M u} u^{-Y} d u \\
& +\frac{1}{\xi^{2}} \int_{0}^{\infty} e^{-M w} w^{-(1+Y)} d w-\frac{1}{\xi^{2}} \int_{0}^{\infty} e^{-(M-i \xi) \nu} \nu^{-(1+Y)} d \nu \\
& =\frac{G^{Y-1} \Gamma(1-Y)}{i \xi}+\frac{G^{Y} \Gamma(-Y)}{\xi^{2}}-\frac{(G+i \xi)^{Y} \Gamma(-Y)}{\xi^{2}}-\frac{M^{Y-1} \Gamma(1-Y)}{i \xi} \\
& +\frac{M^{Y} \Gamma(-Y)}{\xi^{2}}-\frac{(M-i \xi)^{Y} \Gamma(-Y)}{\xi^{2}} .
\end{aligned}
$$

So that, when $\xi \neq 0$, we have the Fourier Transform of $f$ as

$$
\hat{f}(\xi)=\frac{G^{Y-1}-M^{Y-1}}{i \xi} \Gamma(1-Y)-\frac{(G+i \xi)^{Y}+(M-i \xi)^{Y}-G^{Y}-M^{Y}}{\xi^{2}} \Gamma(-Y)
$$

When $\xi=0, \hat{f}(0)$ is just the quadratic variation, we simply refer to (6.18) to see we 
have

$$
\begin{aligned}
\hat{f}(0) & =\frac{1}{2} \int_{-\infty}^{0} \nu^{2} e^{G \nu}(-\nu)^{-(1+Y)} d \nu+\frac{1}{2} \int_{0}^{\infty} \nu^{2} e^{-M \nu} \nu^{-(1+Y)} d \nu \\
& =\frac{1}{2} \int_{0}^{\infty} e^{-G \nu} \nu^{1-Y} d \nu+\frac{1}{2} \int_{0}^{\infty} e^{-M \nu} \nu^{1-Y} d \nu \\
& =\frac{1}{2}\left(G^{Y-2}+M^{Y-2}\right) \Gamma(2-Y) .
\end{aligned}
$$

Putting these results together

$$
\hat{f}(\xi)=\left\{\begin{array}{cl}
\frac{G^{Y-1}-M^{Y-1}}{i \xi} \Gamma(1-Y)-\frac{(G+i \xi)^{Y}+(M-i \xi)^{Y}-G^{Y}-M^{Y}}{\xi^{2}} \Gamma(-Y) & \xi \neq 0, \\
\frac{1}{2}\left(G^{Y-2}+M^{Y-2}\right) \Gamma(2-Y) & \xi=0
\end{array}\right.
$$

Conversely, we should have

$$
f(\nu)=\frac{1}{2 \pi} \int_{-\infty}^{+\infty} e^{-i \xi \nu} \hat{f}(\xi) d \xi
$$

This is carried out using the FFT function in Matlab. The value of $\nu$, or actually the sign of $\nu$ will automatically decide which function we are calculating, $f_{1}$ or $f_{2}$. Specifically, when $\nu<0$, we have $f_{1}(\nu)$; and when $\nu>0$, we have $f_{2}(\nu)$.

Since $f(\nu)$ is a real function, $\hat{f}(\xi)$ is even in its real part and odd in its imaginary part, we thus have

$$
f(\nu)=\text { real part of }\left(\frac{1}{\pi} \int_{0}^{+\infty} e^{-i \xi \nu} \hat{f}(\xi) d \xi\right)
$$

When calculating the integral, we follow the same technique as in [14], using Simpson's Rule and choosing $\eta$ as the spacing for $\xi$, so that $\xi_{j}=\eta(j-1)$ for $j=1, \ldots, \mathcal{N}$; and $\nu_{u}=-b+\lambda *(u-1)$ for $u=1, \ldots, \mathcal{N}$, where $\lambda$ is the spacing for $\nu$ and the range of $\nu_{u}$ is from $-b$ to $b$. The choice of $\lambda$ and $\eta$ must also satisfy the restriction $\lambda \eta=\frac{2 \pi}{\mathcal{N}} \cdot \mathcal{N}$ is usually chosen to be a power of 2 . Now, the discretized 
problem is

$$
f\left(\nu_{u}\right)=\frac{1}{\pi} \sum_{j=1}^{\mathcal{N}} e^{-i \frac{2 \pi}{\mathcal{N}}(j-1)(u-1)} e^{i b \xi_{j}} \hat{f}\left(\xi_{j}\right) \frac{\eta}{3}\left(3+(-1)^{j}-\delta_{j-1}\right)
$$

where $\delta_{n}$ is the Kroneker delta function that is unity for $n=0$ and zero otherwise. The effective upper limit for the integration is $a=\mathcal{N} \eta$. The summation in (6.45) is an exact application of the FFT.

We take $\mathcal{N}=2^{13}, \eta=0.15$ and hence $\lambda \approx 0.005113$. Then calculate $b$ since $b=\lambda(N-1) / 2$. In our case $b \approx 20.9414$. Figure 6.4 is an illustration of (truncated) calculated $f$ versus $\nu$ with a specification of $G=10, M=10$ and $Y=0.5$. 
Figure 6.4: Calculated values of $f$ versus $\nu$ using FFT in Matlab with $G=10$, $M=10$ and $Y=0.5$

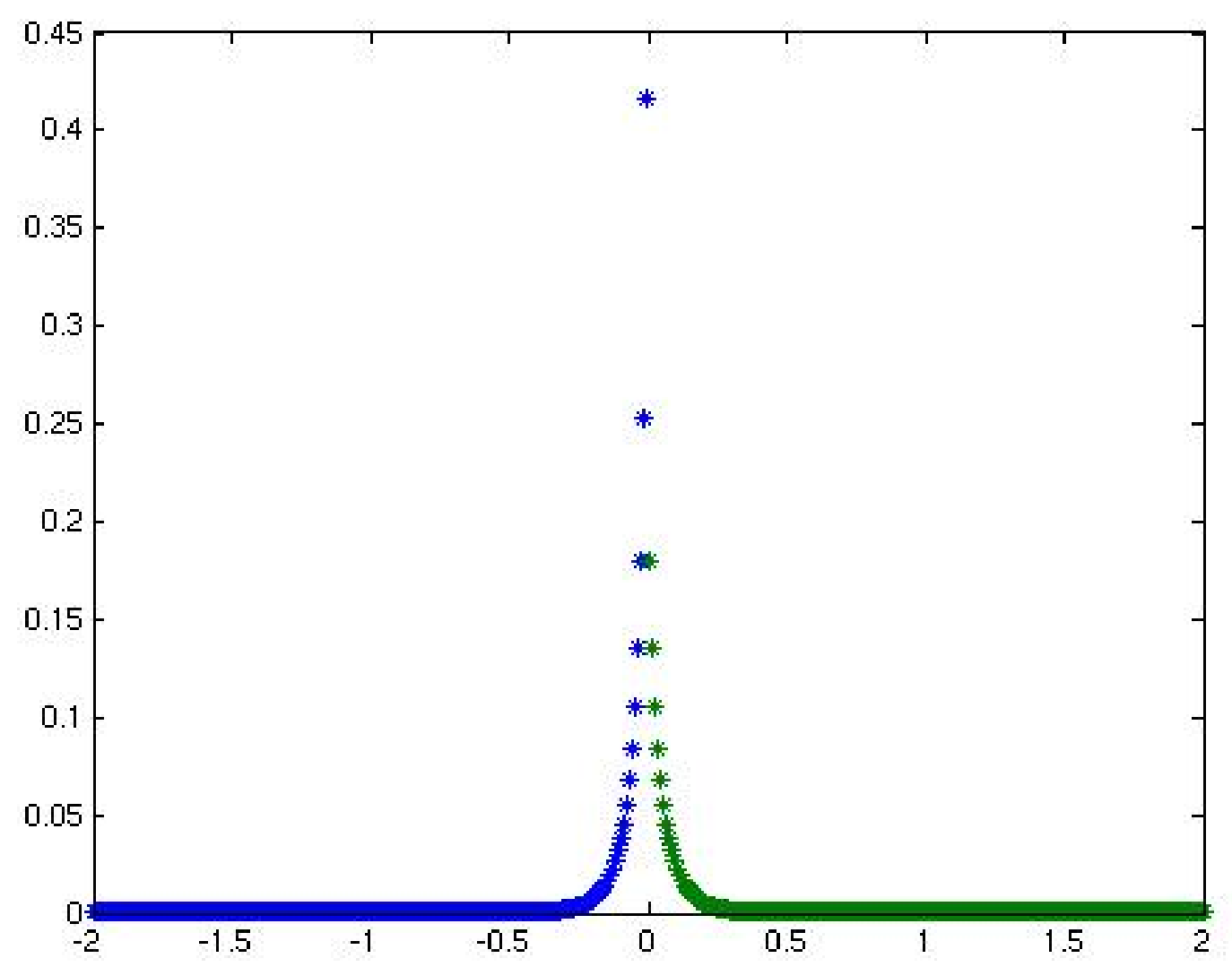

\subsubsection{The System}

We put all the results in section (B.0.8), (B.0.9), (B.0.10) and (B.0.11) together, after careful rearranging and cancellations, in system (6.34), (6.35) and (6.36), we see that during each time step $j, j$ from 2 to $M+1$, we solve a tridiago- 
nal system

$$
\begin{aligned}
(l-r) \lambda C_{i-1, j+1}+ & (1+l \Delta T) C_{i, j+1}+(r-l) \lambda C_{i+1, j+1} \\
= & C_{i, j}+\frac{\Delta T C D e^{\frac{r-l}{\beta} T_{j}}}{2(\Delta k)^{2}}(I+I I) .
\end{aligned}
$$

Function myint is defined as

$$
\begin{aligned}
& \operatorname{myint}(G, x, Y) \\
= & \int_{x}^{\infty} e^{-G w} w^{-(1+Y)} d w \\
\text { or } & \left\{\begin{array}{c}
G^{Y} \Gamma(-Y, G x), \\
\frac{x^{-Y} e^{-G x}}{Y}-\frac{G e^{-G x} x^{1-Y}}{Y(Y-1)}+\frac{G^{Y}}{Y(Y-1)} \Gamma(2-Y, G x), \quad 0<Y<2, Y \neq 1 .
\end{array}\right. \\
= & \left\{\begin{array}{c}
G^{Y} g a m m a i n c(G x,-Y, T A I L), \\
\frac{x^{-Y} e^{-G x}}{Y}-\frac{G e^{-G x} x^{1-Y}}{Y(Y-1)}+\frac{G^{Y}}{Y(Y-1)} \operatorname{gammainc}(G x, 2-Y, T A I L) . \\
0<Y<2, Y \neq 1 .
\end{array}\right.
\end{aligned}
$$

We have two cases depending on different $\hat{\beta}\left(\hat{\beta}=\frac{\beta}{1+2 \beta}\right)$ values. $K_{i}=e^{k_{i}}$ for all $i$. 
When $\hat{\beta} \neq 1$ or $\frac{1}{2}$

$$
\begin{aligned}
& I= \\
& \sum_{m=2}^{i-1}\left[(2-\Delta k) c_{m+1, j}-4 c_{m, j}+(2+\Delta k) c_{m-1, j}\right]\{ \\
& \frac{\hat{\beta} K_{N+1}}{\hat{\beta}-1}\left[\left(K_{m}\right)^{1-\frac{1}{\hat{\beta}}} f_{2}\left(\ln \frac{K_{N+1}}{K_{m}}\right)-\left(K_{m-1}\right)^{1-\frac{1}{\hat{\beta}}} f_{2}\left(\ln \frac{K_{N+1}}{K_{m-1}}\right)\right] \\
& +\frac{\hat{\beta} K_{N+1}}{(\hat{\beta}-1)^{2}}\left[\left(K_{m-1}\right)^{1-\frac{1}{\hat{\beta}}} \operatorname{myint}\left(M, \ln \frac{K_{N+1}}{K_{m-1}}, Y\right)\right. \\
& \left.-\left(K_{m}\right)^{1-\frac{1}{\hat{\beta}}} \operatorname{myint}\left(M, \ln \frac{K_{N+1}}{K_{m}}, Y\right)\right] \\
& +\frac{\hat{\beta}}{2 \hat{\beta}-1}\left[\left(K_{m-1}\right)^{2-\frac{1}{\beta}} \operatorname{myint}\left(M-1, \ln \frac{K_{N+1}}{K_{m-1}}, Y\right)\right. \\
& \left.-\left(K_{m}\right)^{2-\frac{1}{\hat{\beta}}} \operatorname{myint}\left(M-1, \ln \frac{K_{N+1}}{K_{m}}, Y\right)\right] \\
& +\frac{\hat{\beta}^{3} K_{N+1}{ }^{2-\frac{1}{\hat{\beta}}}}{(\hat{\beta}-1)^{2}(2 \hat{\beta}-1)}\left[\operatorname{myint}\left(M+\frac{\hat{\beta}-1}{\hat{\beta}}, \ln \frac{K_{N+1}}{K_{m}}, Y\right)\right. \\
& \left.-\operatorname{myint}\left(M+\frac{\hat{\beta}-1}{\hat{\beta}}, \ln \frac{K_{N+1}}{K_{m-1}}, Y\right)\right] \\
& +\frac{\hat{\beta} K_{i}}{\hat{\beta}-1}\left[\left(K_{m-1}\right)^{1-\frac{1}{\hat{\beta}}} \operatorname{myint}\left(M, \ln \frac{K_{i}}{K_{m-1}}, Y\right)\right. \\
& \left.-\left(K_{m}\right)^{1-\frac{1}{\hat{\beta}}} \operatorname{myint}\left(M, \ln \frac{K_{i}}{K_{m}}, Y\right)\right] \\
& +\frac{\left(K_{i}\right)^{2-\frac{1}{\hat{\beta}}} \hat{\beta}^{2}}{(2 \hat{\beta}-1)(\hat{\beta}-1)}\left[\operatorname{myint}\left(M+\frac{\hat{\beta}-1}{\hat{\beta}}, \ln \frac{K_{i}}{K_{m}}, Y\right)\right. \\
& \left.-\operatorname{myint}\left(M+\frac{\hat{\beta}-1}{\hat{\beta}}, \ln \frac{K_{i}}{K_{m-1}}, Y\right)\right] \\
& +\frac{\hat{\beta}}{2 \hat{\beta}-1}\left[\left(K_{m}\right)^{2-\frac{1}{\hat{\beta}}} \operatorname{myint}\left(M-1, \ln \frac{K_{i}}{K_{m}}, Y\right)\right. \\
& \left.\left.-\left(K_{m-1}\right)^{2-\frac{1}{\hat{\beta}}} \operatorname{myint}\left(M-1, \ln \frac{K_{i}}{K_{m-1}}, Y\right)\right]\right\} \text {, }
\end{aligned}
$$


and

$$
\begin{aligned}
& I I= \\
& \sum_{m=i+1}^{N}\left[(2-\Delta k) c_{m+1, j}-4 c_{m, j}+(2+\Delta k) c_{m-1, j}\right]\{ \\
& \frac{\hat{\beta} K_{1}}{\hat{\beta}-1}\left[\left(K_{m+1}\right)^{1-\frac{1}{\hat{\beta}}} f_{1}\left(\ln \frac{K_{1}}{K_{m+1}}\right)-\left(K_{m}\right)^{1-\frac{1}{\hat{\beta}}} f_{1}\left(\ln \frac{K_{1}}{K_{m}}\right)\right] \\
& +\frac{\hat{\beta} K_{1}}{(\hat{\beta}-1)^{2}}\left[\left(K_{m+1}\right)^{1-\frac{1}{\hat{\beta}}} \operatorname{myint}\left(G, \ln \frac{K_{m+1}}{K_{1}}, Y\right)\right. \\
& \left.-\left(K_{m}\right)^{1-\frac{1}{\hat{\beta}}} \operatorname{myint}\left(G, \ln \frac{K_{m}}{K_{1}}, Y\right)\right] \\
& +\frac{\hat{\beta}}{2 \hat{\beta}-1}\left[\left(K_{m+1}\right)^{2-\frac{1}{\beta}} \operatorname{myint}\left(G+1, \ln \frac{K_{m+1}}{K_{1}}, Y\right)\right. \\
& \left.-\left(K_{m}\right)^{2-\frac{1}{\hat{\beta}}} \operatorname{myint}\left(G+1, \ln \frac{K_{m}}{K_{1}}, Y\right)\right] \\
& +\frac{\hat{\beta}^{3} K_{1}{ }^{2-\frac{1}{\hat{\beta}}}}{(2 \hat{\beta}-1)(\hat{\beta}-1)^{2}}\left[\operatorname{myint}\left(G-\frac{\hat{\beta}-1}{\hat{\beta}}, \ln \frac{K_{m}}{K_{1}}, Y\right)\right. \\
& \left.-\operatorname{myint}\left(G-\frac{\hat{\beta}-1}{\hat{\beta}}, \ln \frac{K_{m+1}}{K_{1}}, Y\right)\right] \\
& +\frac{\hat{\beta} K_{i}}{\hat{\beta}-1}\left[\left(K_{m+1}\right)^{1-\frac{1}{\hat{\beta}}} \operatorname{myint}\left(G, \ln \frac{K_{m+1}}{K_{i}}, Y\right)\right. \\
& \left.-\left(K_{m}\right)^{1-\frac{1}{\hat{\beta}}} \operatorname{myint}\left(G, \ln \frac{K_{m}}{K_{i}}, Y\right)\right] \\
& +\frac{\left(K_{i}\right)^{2-\frac{1}{\hat{\beta}}} \hat{\beta}^{2}}{(2 \hat{\beta}-1)(\hat{\beta}-1)}\left[\operatorname{myint}\left(G-\frac{\hat{\beta}-1}{\hat{\beta}}, \ln \frac{K_{m}}{K_{i}}, Y\right)\right. \\
& \left.-\operatorname{myint}\left(G-\frac{\hat{\beta}-1}{\hat{\beta}}, \ln \frac{K_{m+1}}{K_{i}}, Y\right)\right] \\
& +\frac{\hat{\beta}}{2 \hat{\beta}-1}\left[\left(K_{m}\right)^{2-\frac{1}{\beta}} \operatorname{myint}\left(G+1, \ln \frac{K_{m}}{K_{i}}, Y\right)\right. \\
& \left.\left.-\left(K_{m+1}\right)^{2-\frac{1}{\hat{\beta}}} \operatorname{myint}\left(G+1, \ln \frac{K_{m+1}}{K_{i}}, Y\right)\right]\right\} \text {. }
\end{aligned}
$$


When $\hat{\beta}=1$

$$
\begin{aligned}
I= & \sum_{m=2}^{i-1}\left[(2-\Delta k) c_{m+1, j}-4 c_{m, j}+(2+\Delta k) c_{m-1, j}\right]\{ \\
& K_{N+1}\left(1+\ln K_{m}\right) f_{2}\left(\ln \frac{K_{N+1}}{K_{m}}\right)-K_{N+1}\left(1+\ln K_{m-1}\right) f_{2}\left(\ln \frac{K_{N+1}}{K_{m-1}}\right) \\
+ & K_{i}\left[f_{2}\left(\ln \frac{K_{i}}{K_{m-1}}\right)-f_{2}\left(\ln \frac{K_{i}}{K_{m}}\right)\right] \\
+ & K_{N+1}\left[1+\frac{1}{2}\left(\ln K_{N+1}\right)^{2}-\frac{1}{2}\left(\ln K_{m}\right)^{2}\right] \text { myint }\left(M, \ln \frac{K_{N+1}}{K_{m}}, Y\right) \\
+ & K_{N+1}\left[1+\frac{1}{2}\left(\ln K_{N+1}\right)^{2}-\frac{1}{2}\left(\ln K_{m-1}\right)^{2}\right] \text { myint }\left(M, \ln \frac{K_{N+1}}{K_{m-1}}, Y\right) \\
+ & K_{i}\left[m y i n t\left(M, \ln \frac{K_{i}}{K_{m-1}}, Y\right)-m y i n t\left(M, \ln \frac{K_{i}}{K_{m}}, Y\right)\right] \\
+ & K_{m}\left[\text { myint }\left(M-1, \ln \frac{K_{i}}{K_{m}}, Y\right)-m y i n t\left(M-1, \ln \frac{K_{N+1}}{K_{m}}, Y\right)\right] \\
+ & K_{m-1}\left[\operatorname{myint}\left(M-1, \ln \frac{K_{N+1}}{K_{m-1}}, Y\right)-m y i n t\left(M-1, \ln \frac{K_{i}}{K_{m-1}}, Y\right)\right] \\
+ & K_{N+1} \ln K_{N+1}\left[m y i n t\left(M, \ln \frac{K_{N+1}}{K_{m-1}}, Y-1\right)\right. \\
& \left.-m y i n t\left(M, \ln \frac{K_{N+1}}{K_{m}}, Y-1\right)\right] \\
+ & \frac{1}{2} K_{N+1}\left[\operatorname{myint}\left(M, \ln \frac{K_{N+1}}{K_{m-1}}, Y-2\right)\right. \\
& \left.\left.-m y i n t\left(M, \ln \frac{K_{N+1}}{K_{m}}, Y-2\right)\right]\right\}
\end{aligned}
$$


and

$$
\begin{aligned}
& I I= \\
& \sum_{m=i+1}^{N}\left[(2-\Delta k) c_{m+1, j}-4 c_{m, j}+(2+\Delta k) c_{m-1, j}\right]\{ \\
& K_{1}\left(1+\ln K_{m+1}\right) f_{1}\left(\ln \frac{K_{1}}{K_{m+1}}\right)-K_{1}\left(1+\ln K_{m}\right) f_{1}\left(\ln \frac{K_{1}}{K_{m}}\right) \\
+ & K_{i}\left[f_{1}\left(\ln \frac{K_{i}}{K_{m}}\right)-f_{1}\left(\ln \frac{K_{i}}{K_{m+1}}\right)\right] \\
+ & K_{1}\left[1+\frac{1}{2}\left(\ln K_{1}\right)^{2}-\frac{1}{2}\left(\ln K_{m}\right)^{2}\right] \text { myint }\left(G, \ln \frac{K_{m}}{K_{1}}, Y\right) \\
- & K_{1}\left[1+\frac{1}{2}\left(\ln K_{1}\right)^{2}-\frac{1}{2}\left(\ln K_{m+1}\right)^{2}\right] \text { myint }\left(G, \ln \frac{K_{m+1}}{K_{1}}, Y\right) \\
+ & K_{i}\left[\text { myint }\left(G, \ln \frac{K_{m+1}}{K_{i}}, Y\right)-m y i n t\left(G, \ln \frac{K_{m}}{K_{i}}, Y\right)\right] \\
+ & K_{m+1}\left[\text { myint }\left(G+1, \ln \frac{K_{m+1}}{K_{1}}, Y\right)-m y i n t\left(G+1, \ln \frac{K_{m+1}}{K_{i}}, Y\right)\right] \\
+ & K_{m}\left[\text { myint }\left(G+1, \ln \frac{K_{m}}{K_{i}}, Y\right)-m y i n t\left(G+1, \ln \frac{K_{m}}{K_{1}}, Y\right)\right] \\
- & K_{1} \ln K_{1}\left[\text { myint }\left(G, \ln \frac{K_{m+1}}{K_{1}}, Y-1\right)-\text { myint }\left(G, \ln \frac{K_{m}}{K_{1}}, Y-1\right)\right] \\
- & \left.\frac{1}{2} K_{1}\left[\text { myint }\left(G, \ln \frac{K_{m+1}}{K_{1}}, Y-2\right)-\text { myint }\left(G, \ln \frac{K_{m}}{K_{1}}, Y-2\right)\right]\right\} .(6.5
\end{aligned}
$$




\subsubsection{An Alternative Scheme for Faster Computations}

The evaluation of gamma function is time consuming especially when we have several of them in each loop and many loops during each time step. On top of solving of the PIDE, we are also performing optimization, this makes the program very slow. Hence an alternative faster resolution is needed. We turn back to our discretized system in (6.34):

$$
\begin{array}{r}
(l-r) \lambda c_{i-1, j+1}+(1+l \Delta T) c_{i, j+1}+(r-l) \lambda c_{i+1, j+1} \\
=c_{i, j}+\frac{\Delta T C D e^{\frac{r-l}{\beta} T_{j}}}{2(\Delta k)^{2}}(I+I I),
\end{array}
$$

where

$$
\begin{aligned}
I= & \sum_{m=2}^{i-1}\left[(2-\Delta k) c_{m+1, j}-4 c_{m, j}+(2+\Delta k) c_{m-1, j}\right][ \\
& \left.K_{i} \int_{K_{m-1}}^{K_{m}} U^{-\frac{1}{\beta}-2} f_{2}\left(\ln \frac{K_{i}}{U}\right) d U+\int_{K_{i}}^{K_{N+1}} d U \int_{K_{m-1}}^{K_{m}} W^{-\frac{1}{\beta}-2} f_{2}\left(\ln \frac{U}{W}\right) d W\right],
\end{aligned}
$$

and

$$
\begin{aligned}
I I= & \sum_{m=i+1}^{N}\left[(2-\Delta k) c_{m+1, j}-4 c_{m, j}+(2+\Delta k) c_{m-1, j}\right][ \\
& \left.K_{i} \int_{K_{m}}^{K_{m+1}} U^{-\frac{1}{\beta}-2} f_{1}\left(\ln \frac{K_{i}}{U}\right) d U-\int_{K_{1}}^{K_{i}} d U \int_{K_{m}}^{K_{m+1}} W^{-\frac{1}{\beta}-2} f_{1}\left(\ln \frac{U}{W}\right) d W\right] .
\end{aligned}
$$

All the heavy calculation is focused on the evaluation of the integrals. In previous sections we try our best to find the analytical values for these integrals. In practice, these values (the big second term on the right hand side of (6.51)) are normally of much smaller magnitude relative to the values of $c$ (the first term on the right hand side), and yet we spend so much time calculating them, which seem to be 
unnecessary. Now instead we seek to numerically calculate the integrals. Realizing that once we have the grid in strike $k$ direction ready, we can refine our grid and calculate all the $f$ values (as in section(6.6.4 )) using FFT at all the refined grid points. $f_{1}$ or $f_{2}$ is automatically distinguished by the sign of the input value for receiving $f$. Once all the possible $f$ values are ready, we use a simple trapezoidal rule to calculate the integrals. This numerical approximation is much more efficient and it acquires reasonable accuracy as long as our grid is fine, in fact we have the step size of the refined grid set to be a magnitude less than $10^{-6}$. Most importantly, notice that all the calculation can be done beforehand (before the iteration in the time direction) and all we need is to store the values of these integrals and use them when needed.

We define notation

$$
(i, m) \triangleq \int_{K_{m}}^{K_{m+1}} U^{-\frac{1}{\beta}-2} f\left(\ln \frac{K_{i}}{U}\right) d U=\int_{k_{m}}^{k_{m+1}} e^{-\frac{1+\beta}{\beta} y} f\left(k_{i}-y\right) d y
$$

where

$$
f(\nu)=I_{\{\nu<0\}} f_{1}(\nu)+I_{\{\nu>0\}} f_{2}(\nu) .
$$

This integral value is calculated numerically by using refined grid of $k$ (we use the original step size divided by 1000 to be the new step size). We then store the values of all the integrals in the following matrix 


$$
\text { IntVal }=\left[\begin{array}{cccc}
(1,1) & (1,2) & \ldots & (1, N) \\
(2,1) & (2,2) & \ldots & (2, N) \\
\ldots & & & \\
(N+1,1) & (N+2,2) & \ldots & (N+1, N)
\end{array}\right]
$$

Again, this can all be done before the time iteration begins, which allows us to even use a fully implicit scheme. Using implicit scheme for the first few time steps will also solve the issue of discontinuities in the initial conditions.

Classical convergence results typically rely on smoothness assumptions for the underlying data. However, many financial contracts have discontinuities in the payoff conditions or their derivatives. For the European options we are considering, the initial condition is naturally non-smooth which causes discontinuous first derivatives in the payoffs. Methods for handling discontinuities involves smoothing out the initial condition. Other methods for dealing with initial discontinuities have also been proposed. In Heston and Zhou (2000, [36]), and averaging method discussed in Thomée and Wahlbin (1974, [67]) was used to improve convergence. In Tavella and Randall (2000, [65]), it was suggested that shifting the grid such that discontinuities occur midway between grid points can increase accuracy. Note that this idea is similar to placing nodes equidistantly from a discretely observed barrier (Cheuk and Vorst, 1996, [16]). Perhaps the most general method for handling discontinuities was given in Wahlbin (1980, [68]). Under this method, the initial conditions undergo an $L_{2}$ projection onto the space spanned by a given set of basis functions. To effectively smooth out our initial data, we let the strike prices of interest occur 
not at the mesh nodes but in between them. This method, called shifting the mesh was proposed by Pooley, Forsyth and Vetzal $(2003,[55])$ together with two other methods.

A fully implicit scheme used for the first few time steps could also avoid the problem of discontinuities. Using fully implicit schemes, one only refers to $T=0$ for the time derivatives, so that no derivatives in the $k$ direction are calculated. This is also what Pooley, Forsyth and Vetzal suggest. One may get explicit after a few time steps and this takes care of the issue at the boundary. In fact, we consistently use fully implicit scheme across time since it is a fairly fast computation.

To transform from explicit to implicit, in (6.51) for call options, time index is changed from $j$ to $j+1$ in the far right term, leaving only the value from the previous time step in the equation coming from the time discretization. Hence, we no longer have a tri-diagonal system in each time step.

\subsubsection{Numerical Experiments}

The parameters employed in our study are obtained by calibrating our leveraged Lévy model prices to market data. The forward PIDE formulation allows us to receive option prices for all maturities and all strikes after one execution of the PDE solver. The prices used in the calibration are those of all exchange traded strikes lying within $20 \%$ of the forward price on either side. The data is drawn from CRSP daily option data on $S \& P 500$ for December 31,2003 . The criterion for selection of the parameters is the minimization over the parameter space, $(\beta, \sigma, C, G, M, Y)$, 
of the root mean squared error on an equally weighted basis between market prices and model prices, specifically:

$$
\min _{\beta, \sigma, C, G, M, Y} z=\sqrt{\frac{1}{n} \sum_{i=1}^{n}\left(\text { marketprice }_{i}-\text { modelprice }_{i}(\beta, \sigma, C, G, M, Y)\right)^{2}} .
$$

The S\&P500 spot price on December 31, 2003 was 1110.595285. Market prices used in parameter estimation are for out-of-money options on account of their relative liquidity. More exactly, for strikes below the forward price we use put prices and for strikes above the forward price we use call prices. In total there are 32 calls and 66 puts of 5 maturities. The summary of detail can be found in Table 6.4 .

Table 6.4: Summary of T, $r$ and $q$ for $S \& P 500$ on December 31, 2003

\begin{tabular}{|c|c|c|c|}
\hline$S_{0}$ & $T$ & $r$ & $q$ \\
\hline 1110.595285 & 0.139350188 & 0.010861433 & 0.01615076 \\
1110.595285 & 0.215852921 & 0.011020496 & 0.016844507 \\
1110.595285 & 0.464372957 & 0.011821679 & 0.01599182 \\
1110.595285 & 0.713006837 & 0.012776774 & 0.015944081 \\
1110.595285 & 0.96175456 & 0.014074942 & 0.01647996 \\
\hline
\end{tabular}

The model prices are calculated on a fine mesh of both maturities and strikes and the ones that correspond to the strikes and maturities of the market prices are calculated using cubic spline interpolation. The fully implicit scheme was used 
to solve the forward PIDE as discussed in Section 6.6.6. Table 6.5 contains the calibrated parameters. The fitted data plot is shown in Figure 6.5.

Table 6.5: Calibrated Parameters for S\&P500 on December 31, 2003

\begin{tabular}{|c|c|c|c|c|c|c|}
\hline$\beta$ & $\sigma$ & $C$ & $G$ & $M$ & $Y$ & $z$ \\
\hline 0.1705 & 0.01721 & 14.9970 & 27.7224 & 36.9222 & 1.9505 & 0.3333 \\
\hline
\end{tabular}

The calibrated value for parameter $\beta$ is 0.1705 , which implies that $\alpha=-2.9325$ $\left(\beta=-\frac{1}{2 \alpha}\right)$, negative $\alpha$ value indicates the leverage effect. We argue that since Lévy processes can internally explain both long-tailedness and skewness without the addition of leverage, the estimation of a significant leverage effect is likely just that and not a proxy for other well known and stylized features of the return density.

Comparing the values of $z$, the minimized root mean squared error, which indicates how well the actual data is fitted by prices calculated from our calibrated model, we see that $z=0.3333$ is much smaller than $z=1.4203$ in the BESQ case (Chapter 5). The minimized root mean squared error of all 98 option values is only about thirty three cents, whereas the error in leveraged diffusion with BESQ is one dollar. Again we empirically show that pure jump processes (with infinite activity) generally describe the stock prices' stochastic movement much better compared to the continuous case where Brownian motion is the only driving uncertainty. 
Figure 6.5: Data Fitting For Calls of All Strikes and Maturities on 12-31-2003. Strikes vs. Option prices, $\star$ - Model prices, o - Market prices

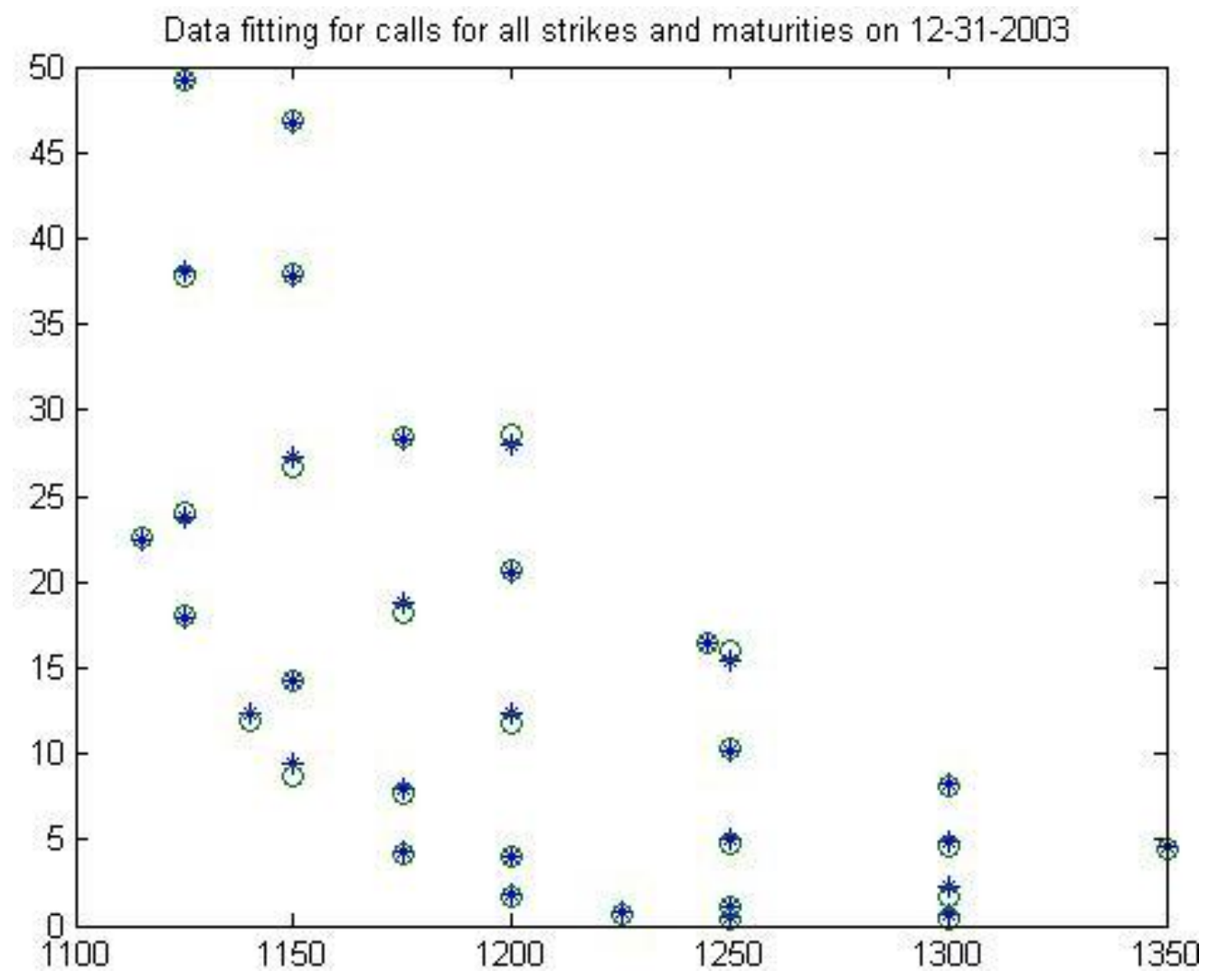




\section{Chapter 7}

\section{CONCLUSION}

It has been observed in Konikov and Madan (2002) that these homogeneous Lévy processes (e.g. NIG, VG, CGMY models) impose strict conditions on the term structure of the risk-neutral variance, skewness, and kurtosis. Specifically, the variance rate is constant over the term. It may be desirable to incorporate a richer behavior by introducing a leverage consideration.

The basic intuition underlying our approach to incorporate leverage in the form of time change arises from the scaling property of semi-stable Markov processes and their one-to-one correspondence to Lévy processes. The scaling property relates changes in scale to changes in time and thus random changes in volatility can alternatively be captured by random changes in time.

The leverage effect is modeled in the context of diffusion by allowing the volatility to be a deterministic function of the spot price and has led to the development of the constant elasticity of variance models and the local volatility models. The analogs in the context of Lévy processes are absent. Stochastic volatility models in the context of Lévy processes have introduced correlation terms to capture this leverage effect but such an approach is relatively indirect. Recently, Carr, Geman, Madan and Yor (2003) have extended the local volatility models to allow for local Lévy models. In this thesis we address leverage directly in the context of a Lévy 
process.

In our study, we build in the leverage effect by introducing a time change dependent on the level of asset and hence affect the expected local volatility in an explicit manner. This is a fairly direct approach in the context of Lévy processes. The continuous case in our study coincides with the development of the constant elasticity of variance models. We however, conduct our investigation in the continuous case through our incorporation of BESQ process as the semi-stable Markov process. The estimated parameters on $S \& P 500$ daily index data show leverage effect with the power being -0.5 . This is the same as many others have documented as for the leverage relation in $S \& P 500$ market. In the pure jump case with underlying time changed Lévy process being specified as CGMY process, we hope to engage the leverage effect as well as the ability of explaining long-tailedness and skewness as already being provided by using such pure jump Lévy process with infinite activity. We show how to implement Generalized Method of Moments in this case to estimate parameters without the assumption of knowing the law of the process.

The development of forward Partial Integro-Differential Equations is under a general setup and shows great advantage over the backward ones. In both the continuous case and the pure jump case, we show how to calibrate our model parameters by solving such forward PIDEs and compare model prices to the market data. Although the numerical approach used in the pure jump case is discussed in the context of CGMY process, it is evident that the approach can be extended to a general frame work indifferent of the choice of Lévy process and shall be similarly carried out where other Lévy processes are specified in our model. 
We expect the subsequent developments will allow for both stochastic volatility and leverage by incorporating stochastic volatility into Lamperti processes. Our method of calibration in the context of such leverage Lévy model and its results shall be then followed by simulation of these models with leverage consideration. It will eventually enable an assessment of the impact of leverage on the valuation of claims otherwise analyzed in a zero leverage context. 


\section{Appendix A}

\section{Evaluation of $\gamma+\omega$}

We want to evaluate $\gamma+\omega=-\int_{R-\{0\}}\left(e^{x}-1-x\right) k_{C G M Y}(x) d x$, where

$$
k_{C G M Y}(x)=\frac{C e^{A x-B|x|}}{|x|^{1+Y}}
$$

is the Lévy density of $X_{C G M Y}$ and $A=\frac{G-M}{2}, B=\frac{G+M}{2} . C>0, G \geq 0, M \geq 0$, and $Y<2$. The condition $Y<2$ is induced by the requirement that Lévy densities integrate $x^{2}$ in the neighborhood of 0 . Let us now begin the evaluation of $\gamma+\omega$ :

$$
\begin{aligned}
& \gamma+\omega \\
= & -\int_{R-\{0\}}\left(e^{x}-1-x\right) k_{C G M Y}(x) d x \\
= & -C\left[\int_{0}^{\infty}\left(e^{x}-1-x\right) \frac{e^{-M x}}{x^{1+Y}} d x+\int_{-\infty}^{0}\left(e^{x}-1-x\right) \frac{e^{G x}}{(-x)^{1+Y}} d x\right] \\
= & C\left[-\int_{0}^{\infty} \frac{e^{-(M-1) x}}{x^{1+Y}} d x+\int_{0}^{\infty} \frac{e^{-M x}}{x^{1+Y}} d x+\int_{0}^{\infty} \frac{e^{-M x}}{x^{Y}} d x\right. \\
& \left.-\int_{-\infty}^{0} \frac{e^{(G+1) x}}{(-x)^{1+Y}} d x+\int_{-\infty}^{0} \frac{e^{G x}}{(-x)^{1+Y}} d x-\int_{-\infty}^{0} \frac{e^{G x}}{(-x)^{Y}} d x\right] \\
= & C\left[-\int_{0}^{\infty} \frac{e^{-(M-1) x}}{x^{1+Y}} d x+\int_{0}^{\infty} \frac{e^{-M x}}{x^{1+Y}} d x+\int_{0}^{\infty} \frac{e^{-M x}}{x^{Y}} d x\right. \\
& \left.-\int_{0}^{\infty} \frac{e^{-(G+1) x}}{x^{1+Y}} d x+\int_{0}^{\infty} \frac{e^{-G x}}{x^{1+Y}} d x-\int_{0}^{\infty} \frac{e^{-G x}}{x^{Y}} d x\right] \\
= & C\left[-(M-1)^{Y} \int_{0}^{\infty} e^{-u} u^{-(1+Y)} d u+M^{Y} \int_{0}^{\infty} e^{-u} u^{-(1+Y)} d u\right. \\
& +M^{Y-1} \int_{0}^{\infty} e^{-u} u^{-Y} d u-(G+1)^{Y} \int_{0}^{\infty} e^{-u} u^{-(1+Y)} d u \\
+ & \left.G^{Y} \int_{0}^{\infty} e^{-u} u^{-(1+Y)} d u-G^{Y-1} \int_{0}^{\infty} e^{-u} u^{-Y} d u\right] .
\end{aligned}
$$


When $Y<0$, all the integrals are finite and we have

$$
\begin{aligned}
& \gamma+\omega \\
= & C \Gamma(1-Y)\left(M^{Y-1}-G^{Y-1}\right)-C \Gamma(-Y)\left[(M-1)^{Y}-M^{Y}+(G+1)^{Y}-G^{Y}\right] .
\end{aligned}
$$

For $0<Y<1$, we evaluate the value of $\gamma+\omega$ as follows.

$$
\begin{aligned}
& \gamma+\omega \\
& =C \lim _{\epsilon \rightarrow 0}\left[-\int_{\epsilon}^{\infty} \frac{e^{-(M-1) x}}{x^{1+Y}} d x+\int_{\epsilon}^{\infty} \frac{e^{-M x}}{x^{1+Y}} d x+\int_{\epsilon}^{\infty} \frac{e^{-M x}}{x^{Y}} d x\right. \\
& \left.-\int_{\epsilon}^{\infty} \frac{e^{-(G+1) x}}{x^{1+Y}} d x+\int_{\epsilon}^{\infty} \frac{e^{-G x}}{x^{1+Y}} d x-\int_{\epsilon}^{\infty} \frac{e^{-G x}}{x^{Y}} d x\right] \\
& =C \lim _{\epsilon \rightarrow 0}\left[-(M-1)^{Y} \int_{(M-1) \epsilon}^{\infty} e^{-u} u^{-(1+Y)} d u+M^{Y} \int_{M \epsilon}^{\infty} e^{-u} u^{-(1+Y)} d u\right. \\
& -(G+1)^{Y} \int_{(G+1) \epsilon}^{\infty} e^{-u} u^{-(1+Y)} d u+G^{Y} \int_{G \epsilon}^{\infty} e^{-u} u^{-(1+Y)} d u \\
& \left.+M^{Y-1} \int_{M \epsilon}^{\infty} e^{-u} u^{-Y} d u-G^{Y-1} \int_{G \epsilon}^{\infty} e^{-u} u^{-Y} d u\right] \\
& =C \lim _{\epsilon \rightarrow 0}\left[\left.\frac{(M-1)^{Y}}{Y} e^{-u} u^{-Y}\right|_{(M-1) \epsilon} ^{\infty}+\frac{(M-1)^{Y}}{Y} \int_{(M-1) \epsilon}^{\infty} e^{-u} u^{-Y} d u\right. \\
& +\left.\frac{M^{Y}}{-Y} e^{-u} u^{-Y}\right|_{M \epsilon} ^{\infty}+\frac{M^{Y}}{-Y} \int_{M \epsilon}^{\infty} e^{-u} u^{-Y} d u \\
& +\left.\frac{(G+1)^{Y}}{Y} e^{-u} u^{-Y}\right|_{(G+1) \epsilon} ^{\infty}+\frac{(G+1)^{Y}}{Y} \int_{(G+1) \epsilon}^{\infty} e^{-u} u^{-Y} d u \\
& \left.+\left.\frac{G^{Y}}{-Y} e^{-u} u^{-Y}\right|_{G \epsilon} ^{\infty}+\frac{G^{Y}}{-Y} \int_{G \epsilon}^{\infty} e^{-u} u^{-Y} d u\right]+C \Gamma(-Y)\left(M^{Y-1}-G^{Y-1}\right) \\
& =C \lim _{\epsilon \rightarrow 0}\left[\frac{e^{-(M-1) \epsilon} \epsilon^{-Y}}{-Y}+\frac{e^{-M \epsilon} \epsilon^{-Y}}{Y}+\frac{e^{-(G+1) \epsilon} \epsilon^{-Y}}{-Y}+\frac{e^{-G \epsilon} \epsilon^{-Y}}{Y}\right] \\
& +\frac{\Gamma(1-Y)}{Y}\left[(M-1)^{Y}-M^{Y}+(G+1)^{Y}-G^{Y}\right] \\
& +C \Gamma(1-Y)\left(M^{Y-1}-G^{Y-1}\right) .
\end{aligned}
$$

In the last line, the quantity in the parenthesis is zero after taking limit and cancellation, so for $0<Y<1$, 


$$
\gamma+\omega=C \Gamma(1-Y)\left[M^{Y-1}-G^{Y-1}+\frac{(M-1)^{Y}}{Y}-\frac{M^{Y}}{Y}+\frac{(G+1)^{Y}}{Y}-\frac{G^{Y}}{Y}\right]
$$

At last, let us consider the case where $1<Y<2$. We evaluate $\gamma+\omega$ from following line (A.1): 


$$
\begin{aligned}
& \gamma+\omega=(A .1) \\
& =C \lim _{\epsilon \rightarrow 0}\left[\frac{(M-1)^{Y}}{Y} \int_{(M-1) \epsilon}^{\infty} e^{-u} u^{-Y} d u+\frac{M^{Y}}{-Y} \int_{M \epsilon}^{\infty} e^{-u} u^{-Y} d u\right. \\
& +\frac{(G+1)^{Y}}{Y} \int_{(G+1) \epsilon}^{\infty} e^{-u} u^{-Y} d u+\frac{G^{Y}}{-Y} \int_{G \epsilon}^{\infty} e^{-u} u^{-Y} d u \\
& +\frac{M^{Y-1}}{1-Y}\left(\left.e^{-u} u^{1-Y}\right|_{M \epsilon} ^{\infty}+\int_{M \epsilon}^{\infty} e^{-u} u^{1-Y} d u\right) \\
& \left.-\frac{G^{Y-1}}{1-Y}\left(\left.e^{-u} u^{1-Y}\right|_{G \epsilon} ^{\infty}+\int_{G \epsilon}^{\infty} e^{-u} u^{1-Y} d u\right)\right] \\
& =\quad C \lim _{\epsilon \rightarrow 0}\left[\frac{(M-1)^{Y}}{Y(1-Y)}\left(\left.e^{-u} u^{1-Y}\right|_{(M-1) \epsilon} ^{\infty}+\int_{(M-1) \epsilon}^{\infty} e^{-u} u^{1-Y} d u\right)\right. \\
& +\frac{M^{Y}}{-Y(1-Y)}\left(\left.e^{-u} u^{1-Y}\right|_{M \epsilon} ^{\infty}+\int_{M \epsilon}^{\infty} e^{-u} u^{1-Y} d u\right) \\
& +\frac{(G+1)^{Y}}{Y(1-Y)}\left(\left.e^{-u} u^{1-Y}\right|_{(G+1) \epsilon} ^{\infty}+\int_{(G+1) \epsilon}^{\infty} e^{-u} u^{1-Y} d u\right) \\
& +\frac{G^{Y}}{-Y(1-Y)}\left(\left.e^{-u} u^{1-Y}\right|_{G \epsilon} ^{\infty}+\int_{G \epsilon}^{\infty} e^{-u} u^{1-Y} d u\right) \\
& +\frac{M^{Y-1}}{1-Y}\left(\left.e^{-u} u^{1-Y}\right|_{M \epsilon} ^{\infty}+\int_{M \epsilon}^{\infty} e^{-u} u^{1-Y} d u\right) \\
& \left.-\frac{G^{Y-1}}{1-Y}\left(\left.e^{-u} u^{1-Y}\right|_{G \epsilon} ^{\infty}+\int_{G \epsilon}^{\infty} e^{-u} u^{1-Y} d u\right)\right] \\
& =C \lim _{\epsilon \rightarrow 0}\left[\frac{\epsilon^{1-Y} e^{-(M-1) \epsilon}-\epsilon^{1-Y} e^{-M \epsilon}+\epsilon^{1-Y} e^{-(G+1) \epsilon}-\epsilon^{1-Y} e^{-G \epsilon}}{-Y(1-Y)}\right. \\
& +\frac{-\epsilon^{1-Y} e^{-M \epsilon}+\epsilon^{1-Y} e^{-G \epsilon}}{1-Y} \\
& +\frac{(M-1)^{Y}}{Y(1-Y)} \Gamma(2-Y)+\frac{M^{Y}}{-Y(1-Y)} \Gamma(2-Y)+\frac{(G+1)^{Y}}{Y(1-Y)} \Gamma(2-Y) \\
& \left.-\frac{G^{Y}}{-Y(1-Y)} \Gamma(2-Y)+\frac{M^{Y-1}}{1-Y} \Gamma(2-Y)-\frac{G^{Y-1}}{1-Y} \Gamma(2-Y)\right] \\
& =C \frac{\Gamma(2-Y)}{Y(1-Y)}\left[(M-1)^{Y}-M^{Y}+(G+1)^{Y}-G^{Y}+Y M^{Y-1}-Y G^{Y-1}\right] .
\end{aligned}
$$


Putting the results together, we have

$$
=\left\{\begin{array}{c}
C\left[\Gamma(1-Y)\left(M^{Y-1}-G^{Y-1}\right)-\Gamma(-Y)\left[(M-1)^{Y}-M^{Y}+(G+1)^{Y}-G^{Y}\right]\right] \\
Y<0 ; \\
C \Gamma(1-Y)\left[M^{Y-1}-G^{Y-1}+\frac{(M-1)^{Y}}{Y}-\frac{M^{Y}}{Y}+\frac{(G+1)^{Y}}{Y}-\frac{G^{Y}}{Y}\right] \\
0<Y<1 ; \\
C \frac{\Gamma(2-Y)}{Y(1-Y)}\left[(M-1)^{Y}-M^{Y}+(G+1)^{Y}-G^{Y}+Y M^{Y-1}-Y G^{Y-1}\right] \\
1<Y<2 .
\end{array}\right.
$$




\section{Appendix B}

\section{Integral Evaluation in the CGMY Case}

In fact when $\hat{\beta}=1$ the evaluation will be different from the evaluation when $\hat{\beta} \neq 1$. We leave this special case to later in section (B.0.12) and let us now calculate these integrals when $\hat{\beta} \neq 1$ one by one in the following sections.

\section{B.0.8 Evaluation of Integral (6.41), when $\hat{\beta} \neq 1$}

In each time step, we need to evaluate for $m=i+1, \ldots, N$

$$
\int_{K_{m}}^{K_{m+1}} U^{-\frac{1}{\hat{\beta}}} f_{1}\left(\ln \frac{K_{i}}{U}\right) d U
$$

Consider a variable change $y=\ln U$, we have

$$
\begin{aligned}
& \int_{K_{m}}^{K_{m+1}} U^{-\frac{1}{\hat{\beta}}} f_{1}\left(\ln \frac{K_{i}}{U}\right) d U \\
= & \int_{\ln K_{m}}^{\ln K_{m+1}} e^{\frac{\hat{\beta}-1}{\hat{\beta}} y} f_{1}\left(\ln K_{i}-y\right) d y \\
= & \frac{\hat{\beta}}{\hat{\beta}-1}\left[\left(K_{m+1}\right)^{\frac{\hat{\beta}-1}{\hat{\beta}}} f_{1}\left(\ln \frac{K_{i}}{K_{m+1}}\right)-\left(K_{m}\right)^{\frac{\hat{\beta}-1}{\hat{\beta}}} f_{1}\left(\ln \frac{K_{i}}{K_{m}}\right)\right. \\
& \left.+\int_{\ln K_{m}}^{\ln K_{m+1}} e^{\frac{\hat{\beta}-1}{\hat{\beta}} y}\left(\int_{-\infty}^{\ln K_{i}-y} e^{G w}(-w)^{-(1+Y)} d w\right) d y\right] \\
= & \frac{\hat{\beta}}{\hat{\beta}-1}\left[\left(K_{m+1}\right)^{\frac{\hat{\beta}-1}{\hat{\beta}}} f_{1}\left(\ln \frac{K_{i}}{K_{m+1}}\right)-\left(K_{m}\right)^{\frac{\hat{\beta}-1}{\hat{\beta}}} f_{1}\left(\ln \frac{K_{i}}{K_{m}}\right)\right] \\
& +\frac{\hat{\beta}}{\hat{\beta}-1} \int_{-\infty}^{\ln \frac{K_{i}}{K_{m}}} d w e^{G w}(-w)^{-(1+Y)} \int_{\ln K_{m}}^{\left(\ln K_{i}-w\right) \wedge \ln K_{m+1}} e^{\frac{\hat{\beta}-1}{\hat{\beta}} y} d y .
\end{aligned}
$$

To evaluate $f_{1}(a)$, where $a$ is a constant, we first analytically calculate the Fourier Transform of

$$
f(\nu)=I_{\{\nu<0\}} f_{1}(\nu)+I_{\{\nu>0\}} f_{2}(\nu),
$$


and then get $f_{1}$ by Fourier Inversion numerically. The evaluation of $f_{1}$ or $f_{2}$ will be determined by the sign of the constant $a$ that we put in function $f$. The detail of the analytical Fourier Transform and how to carry out the inversion numerically will be discussed in section (6.6.4).

We now come to the integral in (B.1). We can achieve the evaluation by using the incomplete gamma function. Specifically, we can first separate the integral into two parts,

$$
\begin{aligned}
& \frac{\hat{\beta}}{\hat{\beta}-1} \int_{-\infty}^{\ln K_{i}-\ln K_{m}} d w e^{G w}(-w)^{-(1+Y)} \int_{\ln K_{m}}^{\left(\ln K_{i}-w\right) \wedge \ln K_{m+1}} e^{\frac{\hat{\beta}-1}{\hat{\beta}} y} d y \\
& =\frac{\hat{\beta}}{\hat{\beta}-1} \int_{-\infty}^{\ln K_{i}-\ln K_{m+1}} d w e^{G w}(-w)^{-(1+Y)} \int_{\ln K_{m}}^{\ln K_{m+1}} e^{\frac{\hat{\beta}-1}{\hat{\beta}} y} d y \\
& +\frac{\hat{\beta}}{\hat{\beta}-1} \int_{\ln K_{i}-\ln K_{m+1}}^{\ln K_{i}-\ln K_{m}} d w e^{G w}(-w)^{-(1+Y)} \int_{\ln K_{m}}^{\ln K_{i}-w} e^{\frac{\hat{\beta}-1}{\hat{\beta}} y} d y \\
& =\left(\frac{\hat{\beta}}{\hat{\beta}-1}\right)^{2} \int_{-\infty}^{\ln K_{i}-\ln K_{m+1}} d w e^{G w}(-w)^{-(1+Y)}\left[\left(K_{m+1}\right)^{\frac{\hat{\beta}-1}{\hat{\beta}}}-\left(K_{m}\right)^{\frac{\hat{\beta}-1}{\hat{\beta}}}\right] \\
& +\left(\frac{\hat{\beta}}{\hat{\beta}-1}\right)^{2} \int_{\ln K_{i}-\ln K_{m+1}}^{\ln K_{i}-\ln K_{m}} d w e^{G w}(-w)^{-(1+Y)}\left[\left(K_{i}\right)^{\frac{\hat{\beta}-1}{\hat{\beta}}} e^{-\frac{\hat{\beta}-1}{\hat{\beta}} w}-\left(K_{m}\right)^{\frac{\hat{\beta}-1}{\hat{\beta}}}\right] \\
& =\left(\frac{\hat{\beta}}{\hat{\beta}-1}\right)^{2}\left[\left(K_{m+1}\right)^{\frac{\hat{\beta}-1}{\hat{\beta}}}-\left(K_{m}\right)^{\frac{\hat{\beta}-1}{\hat{\beta}}}\right] \int_{\ln \frac{K_{m+1}}{K_{i}}}^{+\infty} e^{-G w}(w)^{-(1+Y)} d w \\
& +\left(\frac{\hat{\beta}}{\hat{\beta}-1}\right)^{2}\left[\left(K_{i}\right)^{\frac{\hat{\beta}-1}{\hat{\beta}}} \int_{\ln K_{i}-\ln K_{m+1}}^{\ln K_{i}-\ln K_{m}} e^{\left(G-\frac{\hat{\beta}-1}{\hat{\beta}}\right) w}(-w)^{-(1+Y)} d w\right. \\
& \left.-\left(K_{m}\right)^{\frac{\hat{\beta}-1}{\hat{\beta}}} \int_{\ln K_{i}-\ln K_{m+1}}^{\ln K_{i}-\ln K_{m}} e^{G w}(-w)^{-(1+Y)} d w\right] \\
& =\left(\frac{\hat{\beta}}{\hat{\beta}-1}\right)^{2}\left[\left(K_{m+1}\right)^{\frac{\hat{\beta}-1}{\hat{\beta}}}-\left(K_{m}\right)^{\frac{\hat{\beta}-1}{\hat{\beta}}}\right] \text { integral1 } \\
& +\left(\frac{\hat{\beta}}{\hat{\beta}-1}\right)^{2}[\text { integral } 2-\text { integral } 3] \text {. }
\end{aligned}
$$

It is easily seen that these integral can be expressed by using the upper tail incomplete gamma function, since 


$$
\Gamma(a, x)=\int_{x}^{\infty} e^{-t} t^{a-1} d t
$$

and it is a Matlab function implemented as $\operatorname{gammainc}(X, A, T A I L)$. However, here since the power $-Y$ is not necessarily always positive, we need to be a little more careful. Let us in general work with integral

$$
\int_{x}^{\infty} e^{-G w} w^{-(1+Y)} d w
$$

or after a variable change

$$
G^{Y} \int_{G x}^{\infty} e^{-t} t^{-(1+Y)} d t
$$

When $Y \leq 0$, this is equal to $G^{Y} \Gamma(-Y, G x)$, while $0<Y<2, Y \neq 1$,

$$
\begin{aligned}
& G^{Y} \int_{G x}^{\infty} e^{-t} t^{-(1+Y)} d t \\
= & \frac{-G^{Y}}{Y} \int_{G x}^{\infty} e^{-t} d\left(t^{-Y}\right) \\
= & \frac{-G^{Y}}{Y}\left[-e^{-G x}(G x)^{-Y}+\int_{G x}^{\infty} t^{-Y} e^{-t} d t\right] \\
= & \frac{-G^{Y}}{Y}\left[-e^{-G x}(G x)^{-Y}+\frac{1}{1-Y} \int_{G x}^{\infty} e^{-t} d\left(t^{1-Y}\right)\right] \\
= & \frac{x^{-Y} e^{-G x}}{Y}+\frac{G^{Y}}{(Y-1) Y}\left[-e^{-G x}(G x)^{1-Y}+\int_{G x}^{\infty} t^{1-Y} e^{-t} d t\right] \\
= & \frac{x^{-Y} e^{-G x}}{Y}-\frac{G e^{-G x} x^{1-Y}}{Y(Y-1)}+\frac{G^{Y}}{Y(Y-1)} \Gamma(2-Y, G x) .
\end{aligned}
$$

For the sake of convenience for later use, I will denote the integral (B.2) as function 
$\operatorname{myint}(G, x, a)$ and

$$
\begin{aligned}
& \operatorname{myint}(G, x, Y) \\
= & \int_{x}^{\infty} e^{-G w} w^{-(1+Y)} d w \\
= & \left\{\begin{array}{c}
G^{Y} \Gamma(-Y, G x), \\
\frac{x^{-Y} e^{-G x}}{Y}-\frac{G e^{-G x} x^{1-Y}}{Y(Y-1)}+\frac{G^{Y}}{Y(Y-1)} \Gamma(2-Y, G x) . \quad 0<Y<2, Y \neq 1 . \\
G^{Y} g a m m a i n c(G x,-Y, T A I L),
\end{array} \leq 0,\right. \\
\text { or }= & \left\{\begin{array}{c}
\frac{x^{-Y} e^{-G x}}{Y}-\frac{G e^{-G x} x^{1-Y}}{Y(Y-1)}+\frac{G^{Y}}{Y(Y-1)} \text { gammainc }(G x, 2-Y, T A I L) . \\
0<Y<2, Y \neq 1 .
\end{array}\right.
\end{aligned}
$$

Now, we have

$$
\text { integral1 }=\operatorname{myint}\left(G, \ln \frac{K_{m+1}}{K_{i}}, Y\right)
$$


integral2

$$
\begin{aligned}
& =\left(K_{i}\right)^{\frac{\hat{\beta}-1}{\hat{\beta}}} \int_{\ln K_{i}-\ln K_{m+1}}^{\ln K_{i}-\ln K_{m}} e^{\left(G-\frac{\hat{\beta}-1}{\hat{\beta}}\right) w}(-w)^{-(1+Y)} d w \\
& =\left(K_{i}\right)^{\frac{\hat{\beta}-1}{\hat{\beta}}} \int_{-\infty}^{\ln \frac{K_{i}}{K_{m}}} e^{\left(G-\frac{\hat{\beta}-1}{\hat{\beta}}\right) w}(-w)^{-(1+Y)} d w \\
& -\left(K_{i}\right)^{\frac{\hat{\beta}-1}{\hat{\beta}}} \int_{-\infty}^{\ln \frac{K_{i}}{K_{m+1}}} e^{\left(G-\frac{\hat{\beta}-1}{\hat{\beta}}\right) w}(-w)^{-(1+Y)} d w \\
& =\left(K_{i}\right)^{\frac{\hat{\beta}-1}{\hat{\beta}}} \int_{\ln \frac{K_{m}}{K_{i}}}^{+\infty} e^{-\left(G-\frac{\hat{\beta}-1}{\hat{\beta}}\right) w} w^{-(1+Y)} d w \\
& -\left(K_{i}\right)^{\frac{\hat{\beta}-1}{\hat{\beta}}} \int_{\ln \frac{K_{m+1}}{K_{i}}}^{+\infty} e^{-\left(G-\frac{\hat{\beta}-1}{\hat{\beta}}\right) w} w^{-(1+Y)} d w \\
& =\left(K_{i}\right)^{\frac{\hat{\beta}-1}{\hat{\beta}}} \operatorname{myint}\left(G-\frac{\hat{\beta}-1}{\hat{\beta}}, \ln \frac{K_{m}}{K_{i}}, Y\right) \\
& -\left(K_{i}\right)^{\frac{\hat{\beta}-1}{\hat{\beta}}} \operatorname{myint}\left(G-\frac{\hat{\beta}-1}{\hat{\beta}}, \ln \frac{K_{m+1}}{K_{i}}, Y\right) \\
& =\left(K_{i}\right)^{\frac{\hat{\beta}-1}{\hat{\beta}}}\left[\operatorname{myint}\left(G-\frac{\hat{\beta}-1}{\hat{\beta}}, \ln \frac{K_{m}}{K_{i}}, Y\right)\right. \\
& \left.-\operatorname{myint}\left(G-\frac{\hat{\beta}-1}{\hat{\beta}}, \ln \frac{K_{m+1}}{K_{i}}, Y\right)\right] \text {, }
\end{aligned}
$$

and

integral3

$$
\begin{aligned}
= & \left(K_{m}\right)^{\frac{\hat{\beta}-1}{\hat{\beta}}} \int_{\ln K_{i}-\ln K_{m+1}}^{\ln K_{i}-\ln K_{m}} e^{G w}(-w)^{-(1+Y)} d w \\
= & \left(K_{m}\right)^{\frac{\hat{\beta}-1}{\hat{\beta}}} \int_{-\infty}^{\ln \frac{K_{i}}{K_{m}}} e^{G w}(-w)^{-(1+Y)} d w \\
& -\left(K_{m}\right)^{\frac{\hat{\beta}-1}{\hat{\beta}}} \int_{-\infty}^{\ln \frac{K_{i}}{K_{m+1}}} e^{G w}(-w)^{-(1+Y)} d w \\
= & \left(K_{m}\right)^{\frac{\hat{\beta}-1}{\hat{\beta}}} \int_{\ln \frac{K_{m}}{K_{i}}}^{+\infty} e^{-G w} w^{-(1+Y)} d w-\left(K_{m}\right)^{\frac{\hat{\beta}-1}{\hat{\beta}}} \int_{\ln \frac{K_{m+1}}{K_{i}}}^{+\infty} e^{-G w} w^{-(1+Y)} d w \\
= & \left(K_{m}\right)^{\frac{\hat{\beta}-1}{\hat{\beta}}}\left[\operatorname{myint}\left(G, \ln \frac{K_{m}}{K_{i}}, Y\right)-\operatorname{myint}\left(G, \ln \frac{K_{m+1}}{K_{i}}, Y\right)\right] .
\end{aligned}
$$


So putting things together, we have

$$
\begin{aligned}
& \int_{K_{m}}^{K_{m+1}} U^{-\frac{1}{\hat{\beta}}} f_{1}\left(\ln \frac{K_{i}}{U}\right) d U \\
= & \frac{\hat{\beta}}{\hat{\beta}-1}\left[\left(K_{m+1}\right)^{\frac{\hat{\beta}-1}{\hat{\beta}}} f_{1}\left(\ln \frac{K_{i}}{K_{m+1}}\right)-\left(K_{m}\right)^{\frac{\hat{\beta}-1}{\hat{\beta}}} f_{1}\left(\ln \frac{K_{i}}{K_{m}}\right)\right] \\
& +\left(\frac{\hat{\beta}}{\hat{\beta}-1}\right)^{2}\left\{\left(K_{m+1}\right)^{\frac{\hat{\beta}-1}{\hat{\beta}}} \operatorname{myint}\left(G, \ln \frac{K_{m+1}}{K_{i}}, Y\right)\right. \\
& -\left(K_{m}\right)^{\frac{\hat{\beta}-1}{\hat{\beta}}} \operatorname{myint}\left(G, \ln \frac{K_{m}}{K_{i}}, Y\right) \\
& +\left(K_{i}\right)^{\frac{\hat{\beta}-1}{\hat{\beta}}}\left[\operatorname{myint}\left(G-\frac{\hat{\beta}-1}{\hat{\beta}}, \ln \frac{K_{m}}{K_{i}}, Y\right)\right. \\
& \left.\left.-\operatorname{myint}\left(G-\frac{\hat{\beta}-1}{\hat{\beta}}, \ln \frac{K_{m+1}}{K_{i}}, Y\right)\right]\right\} .
\end{aligned}
$$

\section{B.0.9 Evaluation of Integral (6.42), when $\hat{\beta} \neq 1$}

In each time step, we need to evaluate for $m=2, \ldots, i-1$

$$
\int_{K_{m-1}}^{K_{m}} U^{-\frac{1}{\hat{\beta}}} f_{2}\left(\ln \frac{K_{i}}{U}\right) d U
$$

Similarly as the evaluation in the last section

$$
\begin{aligned}
& \int_{K_{m-1}}^{K_{m}} U^{-\frac{1}{\hat{\beta}}} f_{2}\left(\ln \frac{K_{i}}{U}\right) d U \\
= & \int_{\ln K_{m-1}}^{\ln K_{m}} e^{\frac{\hat{\beta}-1}{\hat{\beta}} y} f_{2}\left(\ln K_{i}-y\right) d y \\
= & \frac{\hat{\beta}}{\hat{\beta}-1}\left[\left(K_{m}\right)^{\frac{\hat{\beta}-1}{\hat{\beta}}} f_{2}\left(\ln \frac{K_{i}}{K_{m}}\right)-\left(K_{m-1}\right)^{\frac{\hat{\beta}-1}{\hat{\beta}}} f_{2}\left(\ln \frac{K_{i}}{K_{m-1}}\right)\right. \\
& \left.-\int_{\ln K_{m-1}}^{\ln K_{m}} e^{\frac{\hat{\beta}-1}{\hat{\beta}} y}\left(\int_{\ln K_{i}-y}^{\infty} e^{-M w} w^{-(1+Y)} d w\right) d y\right] \\
= & \frac{\hat{\beta}}{\hat{\beta}-1}\left[\left(K_{m}\right)^{\frac{\hat{\beta}-1}{\hat{\beta}}} f_{2}\left(\ln \frac{K_{i}}{K_{m}}\right)-\left(K_{m-1}\right)^{\frac{\hat{\beta}-1}{\hat{\beta}}} f_{2}\left(\ln \frac{K_{i}}{K_{m-1}}\right)\right] \\
& -\frac{\hat{\beta}}{\hat{\beta}-1} \int_{\ln K_{i}-\ln K_{m}}^{\infty} d w e^{-M w} w^{-(1+Y)} \int_{\left(\ln K_{i}-w\right) \vee \ln K_{m-1}}^{\ln K_{m}} e^{\frac{\hat{\beta}-1}{\hat{\beta}} y} d y .
\end{aligned}
$$


The evaluation $f_{2}(a)$ when $a$ is a constant, is the same as the evaluation of $f_{1}(a)$. We analytically derive the Fourier Transform of function $f(\nu)=I_{\{\nu<0\}} f_{1}(\nu)+$ $I_{\{\nu>0\}} f_{2}(\nu)$, and then calculate either $f_{1}$ or $f_{2}$ evaluated at certain point by numerical Fourier Inversion. Section (6.6.4) talks about the detail.

We now come to the integral in (B.3). We can achieve the evaluation by using the incomplete gamma function. Specifically, we can first separate the integral into two parts,

$$
\begin{aligned}
& -\frac{\hat{\beta}}{\hat{\beta}-1} \int_{\ln K_{i}-\ln K_{m}}^{\infty} d w e^{-M w} w^{-(1+Y)} \int_{\left(\ln K_{i}-w\right) \ln K_{m-1}}^{\ln K_{m}} e^{\frac{\hat{\beta}-1}{\hat{\beta}} y} d y \\
= & -\frac{\hat{\beta}}{\hat{\beta}-1}\left[\int_{\ln \frac{K_{i}}{K_{m}}}^{\ln \frac{K_{i}}{K_{m}-1}} d w e^{-M w} w^{-(1+Y)} \int_{\ln K_{i}-w}^{\ln K_{m}} e^{\frac{\hat{\beta}-1}{\hat{\beta}} y} d y\right. \\
& \left.+\int_{\ln \frac{K_{i}}{K_{m}-1}}^{+\infty} d w e^{-M w} w^{-(1+Y)} \int_{\ln K_{m-1}}^{\ln K_{m}} e^{\frac{\hat{\beta}-1}{\hat{\beta}} y} d y\right] \\
= & -\left(\frac{\hat{\beta}}{\hat{\beta}-1}\right)^{2}\left[\int_{\ln \frac{K_{i}}{K_{m}}}^{\ln \frac{K_{i}}{K_{m}-1}} d w e^{-M w} w^{-(1+Y)}\left[\left(K_{m}\right)^{\frac{\hat{\beta}-1}{\hat{\beta}}}-\left(K_{i}\right)^{\frac{\hat{\beta}-1}{\hat{\beta}}} e^{-\frac{\hat{\beta}-1}{\hat{\beta}} w}\right]\right. \\
& \left.+\int_{\ln \frac{K_{i}}{K_{m}-1}}^{+\infty} d w e^{-M w} w^{-(1+Y)}\left[\left(K_{m}\right)^{\frac{\hat{\beta}-1}{\hat{\beta}}}-\left(K_{m-1}\right)^{\frac{\hat{\beta}-1}{\hat{\beta}}}\right]\right] \\
= & -\left(\frac{\hat{\beta}}{\hat{\beta}-1}\right)^{2}\left[\left(K_{m}\right)^{\frac{\hat{\beta}-1}{\hat{\beta}}}-\left(K_{m-1}\right)^{\frac{\hat{\beta}-1}{\hat{\beta}}}\right] m y i n t\left(M, \ln \frac{K_{i}}{K_{m-1}}, Y\right) \\
& +\left(K_{m}\right)^{\frac{\hat{\beta}-1}{\hat{\beta}}} \int_{\ln \frac{K_{i}}{K_{m}}}^{\ln \frac{K_{i}}{K_{m}-1}} e^{-M w} w^{-(1+Y)} d w \\
& \left.-\left(K_{i}\right)^{\frac{\hat{\beta}-1}{\hat{\beta}}} \int_{\ln \frac{K_{i}}{K_{m}}}^{\ln \frac{K_{i}}{K_{m}-1}} e^{-\left(M+\frac{\hat{\beta}-1}{\hat{\beta}}\right) w} w^{-(1+Y)} d w\right],
\end{aligned}
$$

where

$$
\begin{aligned}
& \int_{\ln \frac{K_{i}}{K_{m}}}^{\ln \frac{K_{i}}{K_{m}-1}} e^{-M w} w^{-(1+Y)} d w \\
& =\int_{\ln \frac{K_{i}}{K m}}^{+\infty} e^{-M w} w^{-(1+Y)} d w-\int_{\ln \frac{K_{i}}{K_{m}-1}}^{+\infty} e^{-M w} w^{-(1+Y)} d w \\
& =\operatorname{myint}\left(M, \ln \frac{K_{i}}{K_{m}}, Y\right)-\operatorname{myint}\left(M, \ln \frac{K_{i}}{K_{m-1}}, Y\right) \text {, }
\end{aligned}
$$


and

$$
\begin{aligned}
& \int_{\ln \frac{K_{i}}{K_{m}}}^{\ln \frac{K_{i}}{K_{m-1}}} e^{-\left(M+\frac{\hat{\beta}-1}{\hat{\beta}}\right) w} w^{-(1+Y)} d w \\
= & \int_{\ln \frac{K_{i}}{K_{m}}}^{+\infty} e^{-\left(M+\frac{\hat{\beta}-1}{\hat{\beta}}\right) w} w^{-(1+Y)} d w-\int_{\ln \frac{K_{i}}{K_{m-1}}}^{+\infty} e^{-\left(M+\frac{\hat{\beta}-1}{\hat{\beta}}\right) w} w^{-(1+Y)} d w \\
= & \operatorname{myint}\left(M+\frac{\hat{\beta}-1}{\hat{\beta}}, \ln \frac{K_{i}}{K_{m}}, Y\right)-\operatorname{myint}\left(M+\frac{\hat{\beta}-1}{\hat{\beta}}, \ln \frac{K_{i}}{K_{m-1}}, Y\right) .
\end{aligned}
$$

So putting things together, we have

$$
\begin{aligned}
& \int_{K_{m-1}}^{K_{m}} U^{-\frac{1}{\hat{\beta}}} f_{2}\left(\ln \frac{K_{i}}{U}\right) d U \\
= & \frac{\hat{\beta}}{\hat{\beta}-1}\left[\left(K_{m}\right)^{\frac{\hat{\beta}-1}{\hat{\beta}}} f_{2}\left(\ln \frac{K_{i}}{K_{m}}\right)-\left(K_{m-1}\right)^{\frac{\hat{\beta}-1}{\hat{\beta}}} f_{2}\left(\ln \frac{K_{i}}{K_{m-1}}\right)\right] \\
& -\left(\frac{\hat{\beta}}{\hat{\beta}-1}\right)^{2}\left\{\left(K_{m}\right)^{\frac{\hat{\beta}-1}{\hat{\beta}}} \operatorname{myint}\left(M, \ln \frac{K_{i}}{K_{m}}, Y\right)\right. \\
& -\left(K_{m-1}\right)^{\frac{\hat{\beta}-1}{\hat{\beta}}} \operatorname{myint}\left(M, \ln \frac{K_{i}}{K_{m-1}}, Y\right) \\
& -\left(K_{i}\right)^{\frac{\hat{\beta}-1}{\hat{\beta}}}\left[\text { myint }\left(M+\frac{\hat{\beta}-1}{\hat{\beta}}, \ln \frac{K_{i}}{K_{m}}, Y\right)\right. \\
& \left.\left.- \text { myint }\left(M+\frac{\hat{\beta}-1}{\hat{\beta}}, \ln \frac{K_{i}}{K_{m-1}}, Y\right)\right]\right\} .
\end{aligned}
$$

\section{B.0.10 Evaluation of Integral (6.43), when $\hat{\beta} \neq 1$}

In each time step, we need to evaluate for $m=2, \ldots, i-1$

$$
\int_{K_{i}}^{K_{N+1}} d U \int_{K_{m-1}}^{K_{m}} W^{-\frac{1}{\beta}} f_{2}\left(\ln \frac{U}{W}\right) d W .
$$


Rearrange the integral, we have

$$
\begin{aligned}
& \int_{K_{i}}^{K_{N+1}} d U \int_{K_{m-1}}^{K_{m}} W^{-\frac{1}{\hat{\beta}}} f_{2}\left(\ln \frac{U}{W}\right) d W \\
& =\int_{K_{m-1}}^{K_{m}} W^{-\frac{1}{\hat{\beta}}} d W \int_{K_{i}}^{K_{N+1}} f_{2}\left(\ln \frac{U}{W}\right) d U \\
& \ln \frac{U}{\underline{\underline{W}}}=t \quad \int_{K_{m-1}}^{K_{m}} W^{-\frac{1}{\hat{\beta}}} d W \int_{\ln \frac{K_{i}}{W}}^{\ln \frac{K_{N+1}}{W}} f_{2}(t) W e^{t} d t \\
& =\int_{K_{m-1}}^{K_{m}} W^{1-\frac{1}{\hat{\beta}}} d W \int_{\ln \frac{K_{i}}{W}}^{\ln \frac{K_{N+1}}{W}} f_{2}(t) e^{t} d t \\
& =\frac{1}{2-\frac{1}{\hat{\beta}}}\left[\left(K_{m}\right)^{2-\frac{1}{\hat{\beta}}} \int_{\ln \frac{K_{i}}{K_{m}}}^{\ln \frac{K_{N+1}}{K_{m}}} f_{2}(t) e^{t} d t-\left(K_{m-1}\right)^{2-\frac{1}{\hat{\beta}}} \int_{\ln \frac{K_{i}}{K_{m-1}}}^{\ln \frac{K_{N+1}}{K_{m-1}}} f_{2}(t) e^{t} d t\right. \\
& +\int_{K_{m-1}}^{K_{m}} W^{2-\frac{1}{\hat{\beta}}} f_{2}\left(\ln \frac{K_{i}}{W}\right) \frac{K_{i}}{W} \frac{W}{K_{i}} K_{i}\left(-\frac{1}{W^{2}}\right) d W \\
& \left.-\int_{K_{m-1}}^{K_{m}} W^{2-\frac{1}{\hat{\beta}}} f_{2}\left(\ln \frac{K_{N+1}}{W}\right) \frac{K_{N+1}}{W} \frac{W}{K_{N+1}} K_{N+1}\left(-\frac{1}{W^{2}}\right) d W\right] \\
& =\frac{1}{2-\frac{1}{\hat{\beta}}}\left[\left(K_{m}\right)^{2-\frac{1}{\hat{\beta}}} \int_{\ln \frac{K_{i}}{K_{m}}}^{\ln \frac{K_{N+1}}{K_{m}}} f_{2}(t) e^{t} d t-\left(K_{m-1}\right)^{2-\frac{1}{\hat{\beta}}} \int_{\ln \frac{K_{i}}{K_{m-1}}}^{\ln \frac{K_{N+1}}{K_{m-1}}} f_{2}(t) e^{t} d t\right. \\
& \left.-K_{i} \int_{K_{m-1}}^{K_{m}} W^{-\frac{1}{\hat{\beta}}} f_{2}\left(\ln \frac{K_{i}}{W}\right) d W+K_{N+1} \int_{K_{m-1}}^{K_{m}} W^{-\frac{1}{\hat{\beta}}} f_{2}\left(\ln \frac{K_{N+1}}{W}\right) d W\right] .
\end{aligned}
$$

Here $\hat{\beta} \neq \frac{1}{2}$, this is true since $\hat{\beta}$ is in fact $\frac{\beta}{1+2 \beta}$. Noticing that integral

$$
\int_{K_{m}}^{K_{m+1}} W^{-\frac{1}{\hat{\beta}}} f_{2}\left(\ln \frac{K_{i}}{W}\right) d W
$$

has been evaluated in section (B.0.9), we only need to evaluate two other integrals, 
which are

$$
\begin{aligned}
& \int_{\ln \frac{K_{i}}{K_{m}}}^{\ln \frac{K_{N+1}}{K_{m}}} f_{2}(t) e^{t} d t \\
= & \frac{K_{N+1}}{K_{m}} f_{2}\left(\ln \frac{K_{N+1}}{K_{m}}\right)-\frac{K_{i}}{K_{m}} f_{2}\left(\ln \frac{K_{i}}{K_{m}}\right)+\int_{\ln \frac{K_{i}}{K_{m}}}^{\ln \frac{K_{N+1}}{K_{m}}} e^{t}\left(\int_{t}^{\infty} e^{-M w} w^{-(1+Y)} d w\right) d t \\
= & \frac{K_{N+1}}{K_{m}} f_{2}\left(\ln \frac{K_{N+1}}{K_{m}}\right)-\frac{K_{i}}{K_{m}} f_{2}\left(\ln \frac{K_{i}}{K_{m}}\right)+\frac{K_{N+1}}{K_{m}} \int_{\ln \frac{K_{N+1}}{K_{m}}}^{\infty} e^{-M w} w^{-(1+Y)} d w \\
= & \frac{K_{N}}{K_{m}} \int_{\ln }^{\infty} \frac{K_{i}}{K_{m}} f_{2}\left(\ln \frac{K_{N+1}}{K_{m}}\right)-\frac{K_{i}}{K_{m}} f_{2}\left(\ln \frac{K_{i}}{K_{m}}\right) \\
& +\frac{K_{N+1}}{K_{m}} m y i n t\left(M, \ln \frac{K_{N+1}}{K_{m}}, Y\right)-\frac{K_{i}}{K_{m}} e^{t} e^{-M t} t^{-(1+Y)} d t \\
& +m y i n t\left(M i n t\left(M, \ln \frac{K_{i}}{K_{m}}, Y\right)\right. \\
& \left.m-1, \ln \frac{K_{i}}{K_{m}}, Y\right)-m y i n t\left(M-1, \ln \frac{K_{N+1}}{K_{m}}, Y\right)
\end{aligned}
$$

and

$$
\begin{aligned}
& \int_{\ln \frac{K_{i}}{K_{m-1}}}^{\ln \frac{K_{N+1}}{K_{m-1}}} f_{2}(t) e^{t} d t \\
= & \frac{K_{N+1}}{K_{m-1}} f_{2}\left(\ln \frac{K_{N+1}}{K_{m-1}}\right)-\frac{K_{i}}{K_{m-1}} f_{2}\left(\ln \frac{K_{i}}{K_{m-1}}\right) \\
& +\frac{K_{N+1}}{K_{m-1}} \operatorname{myint}\left(M, \ln \frac{K_{N+1}}{K_{m-1}}, Y\right)-\frac{K_{i}}{K_{m-1}} \operatorname{myint}\left(M, \ln \frac{K_{i}}{K_{m-1}}, Y\right) \\
& + \text { myint }\left(M-1, \ln \frac{K_{i}}{K_{m-1}}, Y\right)-\operatorname{myint}\left(M-1, \ln \frac{K_{N+1}}{K_{m-1}}, Y\right) .
\end{aligned}
$$

\section{B.0.11 Evaluation of Integral (6.44), when $\hat{\beta} \neq 1$}

In each time step, we need to evaluate for $m=i+1, \ldots, N$

$$
\int_{K_{1}}^{K_{i}} d U \int_{K_{m}}^{K_{m+1}} W^{-\frac{1}{\hat{\beta}}} f_{1}\left(\ln \frac{U}{W}\right) d W
$$


Rearrange the integral, we have

$$
\begin{aligned}
& \int_{K_{1}}^{K_{i}} d U \int_{K_{m}}^{K_{m+1}} W^{-\frac{1}{\hat{\beta}}} f_{1}\left(\ln \frac{U}{W}\right) d W \\
=\quad & \int_{K_{m}}^{K_{m+1}} W^{-\frac{1}{\hat{\beta}}} d W \int_{K_{1}}^{K_{i}} f_{1}\left(\ln \frac{U}{W}\right) d U \\
\ln \frac{U}{\underline{\underline{W}}=t} & \int_{K_{m}}^{K_{m+1}} W^{-\frac{1}{\hat{\beta}}} d W \int_{\ln \frac{K_{1}}{W}}^{\ln \frac{K_{i}}{W}} f_{1}(t) W e^{t} d t \\
= & \int_{K_{m}}^{K_{m+1}} W^{1-\frac{1}{\hat{\beta}}} d W \int_{\ln \frac{K_{1}}{W}}^{\ln \frac{K_{i}}{W}} f_{1}(t) e^{t} d t \\
= & \frac{1}{2-\frac{1}{\hat{\beta}}}\left[\left(K_{m+1}\right)^{2-\frac{1}{\hat{\beta}}} \int_{\ln \frac{K_{1}}{K_{m+1}}}^{\ln \frac{K_{i}}{K_{m+1}}} f_{1}(t) e^{t} d t-\left(K_{m}\right)^{2-\frac{1}{\hat{\beta}}} \int_{\ln \frac{K_{1}}{K_{m}}}^{\ln \frac{K_{i}}{K_{m}}} f_{1}(t) e^{t} d t\right. \\
& -\int_{K_{m}}^{K_{m+1}} W^{2-\frac{1}{\hat{\beta}}} f_{1}\left(\ln \frac{K_{i}}{W}\right) \frac{K_{i}}{W} \frac{W}{K_{i}} K_{i}\left(-\frac{1}{W^{2}}\right) d W \\
& \left.+\int_{K_{m}}^{K_{m+1}} W^{2-\frac{1}{\hat{\beta}}} f_{1}\left(\ln \frac{K_{1}}{W}\right) \frac{K_{1}}{W} \frac{W}{K_{1}} K_{1}\left(-\frac{1}{W^{2}}\right) d W\right] \\
& \frac{1}{2-\frac{1}{\hat{\beta}}}\left[\left(K_{m+1}\right)^{2-\frac{1}{\hat{\beta}}} \int_{\ln \frac{K_{1}}{K_{m+1}}}^{\ln \frac{K_{i}}{K_{m+1}}} f_{1}(t) e^{t} d t-\left(K_{m}\right)^{2-\frac{1}{\hat{\beta}}} \int_{\ln \frac{K_{1}}{K_{m}}}^{\ln \frac{K_{i}}{K_{m}}} f_{1}(t) e^{t} d t\right. \\
+ & \left.K_{i} \int_{K_{m}}^{K_{m+1}} W^{-\frac{1}{\hat{\beta}}} f_{1}\left(\ln \frac{K_{i}}{W}\right) d W-K_{1} \int_{K_{m}} W^{-\frac{1}{\hat{\beta}}} f_{1}\left(\ln \frac{K_{1}}{W}\right) d W\right] .
\end{aligned}
$$

Here again $\hat{\beta} \neq \frac{1}{2}$. Noticing that integral $\int_{K_{m}}^{K_{m+1}} W^{-\frac{1}{\hat{\beta}}} f_{1}\left(\ln \frac{K_{i}}{W}\right) d W$ has been evalu- 
ated in section (B.0.8), we only need to evaluate two other integrals, which are

$$
\begin{aligned}
& \int_{\ln \frac{K_{1}}{K_{m+1}}}^{\ln \frac{K_{i}}{K_{m+1}}} f_{1}(t) e^{t} d t \\
= & \frac{K_{i}}{K_{m+1}} f_{1}\left(\ln \frac{K_{i}}{K_{m+1}}\right)-\frac{K_{1}}{K_{m+1}} f_{1}\left(\ln \frac{K_{1}}{K_{m+1}}\right) \\
& -\int_{\ln \frac{K_{1}}{K_{m+1}}}^{\ln \frac{K_{i}}{K_{m+1}}} e^{t}\left(\int_{-\infty}^{t} e^{G w}(-w)^{-(1+Y)} d w\right) d t \\
= & \frac{K_{i}}{K_{m+1}} f_{1}\left(\ln \frac{K_{i}}{K_{m+1}}\right)-\frac{K_{1}}{K_{m+1}} f_{1}\left(\ln \frac{K_{1}}{K_{m+1}}\right)-\frac{K_{i}}{K_{m+1}} \int_{-\infty}^{\ln \frac{K_{i}}{K_{m+1}}} e^{G w}(-w)^{-(1+Y)} d w \\
& +\frac{K_{1}}{K_{m+1}} \int_{-\infty}^{\ln \frac{K_{1}}{K_{m+1}}} e^{G w}(-w)^{-(1+Y)} d w+\int_{\ln } \frac{K_{1}}{K_{m+1}} e^{(1+G) t}(-t)^{-(1+Y)} d t \\
= & \frac{K_{i}}{K_{m+1}} f_{1}\left(\ln \frac{K_{i}}{K_{m+1}}\right)-\frac{K_{1}}{K_{m+1}} f_{1}\left(\ln \frac{K_{1}}{K_{m+1}}\right) \\
& -\frac{K_{i}}{K_{m+1}} m y i n t\left(G, \ln \frac{K_{m+1}}{K_{i}}, Y\right)+\frac{K_{1}}{K_{m+1}} m y i n t\left(G, \ln \frac{K_{m+1}}{K_{1}}, Y\right) \\
& +m y i n t\left(G+1, \ln \frac{K_{m+1}}{K_{i}}, Y\right)-m y i n t\left(G+1, \ln \frac{K_{m+1}}{K_{1}}, Y\right),
\end{aligned}
$$

and

$$
\begin{aligned}
& \int_{\ln \frac{K_{1}}{K_{m}}}^{\ln \frac{K_{i}}{K_{m}}} f_{1}(t) e^{t} d t \\
= & \frac{K_{i}}{K_{m}} f_{1}\left(\ln \frac{K_{i}}{K_{m}}\right)-\frac{K_{1}}{K_{m}} f_{1}\left(\ln \frac{K_{1}}{K_{m}}\right) \\
& -\frac{K_{i}}{K_{m}} \operatorname{myint}\left(G, \ln \frac{K_{m}}{K_{i}}, Y\right)+\frac{K_{1}}{K_{m}} \operatorname{myint}\left(G, \ln \frac{K_{m}}{K_{1}}, Y\right) \\
& + \text { myint }\left(G+1, \ln \frac{K_{m}}{K_{i}}, Y\right)-\operatorname{myint}\left(G+1, \ln \frac{K_{m}}{K_{1}}, Y\right) .
\end{aligned}
$$


B.0.12 Evaluation of Integrals (6.41-6.44) when $\hat{\beta}=1$

$$
\begin{aligned}
& \int_{K_{m}}^{K_{m+1}} U^{-1} f_{1}\left(\ln \frac{K_{i}}{U}\right) d U \\
& =\int_{\ln K_{m}}^{\ln K_{m+1}} d y f_{1}\left(\ln K_{i}-y\right) \\
& =\ln K_{m+1} f_{1}\left(\ln \frac{K_{i}}{K_{m+1}}\right)-\ln K_{m} f_{1}\left(\ln \frac{K_{i}}{K_{m}}\right) \\
& +\int_{\ln K_{m}}^{\ln K_{m+1}} y\left(\int_{-\infty}^{\ln K_{i}-y} e^{G w}(-w)^{-(1+Y)} d w\right) d y \\
& =\ln K_{m+1} f_{1}\left(\ln \frac{K_{i}}{K_{m+1}}\right)-\ln K_{m} f_{1}\left(\ln \frac{K_{i}}{K_{m}}\right) \\
& +\frac{1}{2}\left(\ln K_{m+1}\right)^{2} \int_{-\infty}^{\ln \frac{K_{i}}{K_{m+1}}} e^{G w}(-w)^{-(1+Y)} d w \\
& -\frac{1}{2}\left(\ln K_{m}\right)^{2} \int_{-\infty}^{\ln \frac{K_{i}}{K_{m}}} e^{G w}(-w)^{-(1+Y)} d w \\
& +\frac{1}{2} \int_{\ln K_{m}}^{\ln K_{m+1}} y^{2} e^{G\left(\ln K_{i}-y\right)}\left(y-\ln K_{i}\right)^{-(1+Y)} d y \\
& =\ln K_{m+1} f_{1}\left(\ln \frac{K_{i}}{K_{m+1}}\right)-\ln K_{m} f_{1}\left(\ln \frac{K_{i}}{K_{m}}\right)+\frac{1}{2}\left(\ln K_{m+1}\right)^{2} m y i n t\left(G, \ln \frac{K_{m+1}}{K_{i}}, Y\right) \\
& -\frac{1}{2}\left(\ln K_{m}\right)^{2} \operatorname{myint}\left(G, \ln \frac{K_{m}}{K_{i}}, Y\right) \\
& -\frac{1}{2} \int_{\ln \frac{K_{i}}{K_{m}}}^{\ln \frac{K_{i}}{K_{m+1}}}\left[\left(\ln K_{i}\right)^{2}-2 t \ln K_{i}+t^{2}\right] e^{G t}(-t)^{-(1+Y)} d t \\
& =\ln K_{m+1} f_{1}\left(\ln \frac{K_{i}}{K_{m+1}}\right)-\ln K_{m} f_{1}\left(\ln \frac{K_{i}}{K_{m}}\right)+\frac{1}{2}\left(\ln K_{m+1}\right)^{2} m y i n t\left(G, \ln \frac{K_{m+1}}{K_{i}}, Y\right) \\
& -\frac{1}{2}\left(\ln K_{m}\right)^{2} \text { myint }\left(G, \ln \frac{K_{m}}{K_{i}}, Y\right)-\frac{1}{2}\left(\ln K_{i}\right)^{2}\left[\operatorname{myint}\left(G, \ln \frac{K_{m+1}}{K_{i}}, Y\right)\right. \\
& \left.-\operatorname{myint}\left(G, \ln \frac{K_{m}}{K_{i}}, Y\right)\right]-\ln K_{i}\left[\operatorname{myint}\left(G, \ln \frac{K_{m+1}}{K_{i}}, Y-1\right)\right. \\
& \left.-\operatorname{myint}\left(G, \ln \frac{K_{m}}{K_{i}}, Y-1\right)\right] \\
& -\frac{1}{2}\left[\operatorname{myint}\left(G, \ln \frac{K_{m+1}}{K_{i}}, Y-2\right)-\operatorname{myint}\left(G, \ln \frac{K_{m}}{K_{i}}, Y-2\right)\right] \text {, }
\end{aligned}
$$




$$
\begin{aligned}
& \int_{K_{m}}^{K_{m+1}} U^{-1} f_{1}\left(\ln \frac{K_{i}}{U}\right) d U \\
= & \ln K_{m+1} f_{1}\left(\ln \frac{K_{i}}{K_{m+1}}\right)-\ln K_{m} f_{1}\left(\ln \frac{K_{i}}{K_{m}}\right) \\
& +\frac{1}{2}\left[\left(\ln K_{m+1}\right)^{2}-\left(\ln K_{i}\right)^{2}\right] \operatorname{myint}\left(G, \ln \frac{K_{m+1}}{K_{i}}, Y\right) \\
& +\frac{1}{2}\left[\left(\ln K_{i}\right)^{2}-\left(\ln K_{m}\right)^{2}\right] \operatorname{myint}\left(G, \ln \frac{K_{m}}{K_{i}}, Y\right) \\
& -\ln K_{i}\left[\operatorname{myint}\left(G, \ln \frac{K_{m+1}}{K_{i}}, Y-1\right)-\operatorname{myint}\left(G, \ln \frac{K_{m}}{K_{i}}, Y-1\right)\right] \\
& -\frac{1}{2}\left[\operatorname{myint}\left(G, \ln \frac{K_{m+1}}{K_{i}}, Y-2\right)-\operatorname{myint}\left(G, \ln \frac{K_{m}}{K_{i}}, Y-2\right)\right],
\end{aligned}
$$

and

$$
\begin{aligned}
& \int_{K_{m-1}}^{K_{m}} U^{-1} f_{2}\left(\ln \frac{K_{i}}{U}\right) d U \\
= & \int_{\ln K_{m-1}}^{\ln K_{m}} d y f_{2}\left(\ln K_{i}-y\right) \\
= & \ln K_{m} f_{2}\left(\ln \frac{K_{i}}{K_{m}}\right)-\ln K_{m-1} f_{2}\left(\ln \frac{K_{i}}{K_{m-1}}\right) \\
& -\int_{\ln K_{m-1}}^{\ln K_{m}} y\left(\int_{\ln K_{i}-y}^{\infty} e^{-M w} w^{-(1+Y)} d w\right) d y \\
= & \ln K_{m} f_{2}\left(\ln \frac{K_{i}}{K_{m}}\right)-\ln K_{m-1} f_{2}\left(\ln \frac{K_{i}}{K_{m-1}}\right)-\frac{1}{2}\left(\ln K_{m}\right)^{2} \int_{\ln \frac{K_{i}}{K_{m}}}^{\infty} e^{-M w} w^{-(1+Y)} d w \\
& +\frac{1}{2}\left(\ln K_{m-1}\right)^{2} \int_{\ln \frac{K_{i}}{K_{m-1}}}^{\infty} e^{-M w} w^{-(1+Y)} d w \\
& +\frac{1}{2} \int_{\ln K_{m-1}}^{\ln K_{m}} y^{2} e^{-M\left(\ln K_{i}-y\right)}\left(\ln K_{i}-y\right)^{-(1+Y)} d y \\
= & \ln K_{m} f_{2}\left(\ln \frac{K_{i}}{K_{m}}\right)-\ln K_{m-1} f_{2}\left(\ln \frac{K_{i}}{K_{m-1}}\right)-\frac{1}{2}\left(\ln K_{m}\right)^{2} m y i n t\left(M, \ln \frac{K_{i}}{K_{m}}, Y\right) \\
& +\frac{1}{2}\left(\ln K_{m-1}\right)^{2} m y i n t\left(M, \ln \frac{K_{i}}{K_{m-1}}, Y\right) \\
& -\frac{1}{2} \int_{\ln \frac{K_{i}}{K_{m}-1}}^{\ln \frac{K_{i}}{K_{m}}}\left[\left(\ln K_{i}\right)^{2}-2 t \ln K_{i}+t^{2}\right] e^{-M t} t^{-(1+Y)} d t .
\end{aligned}
$$


Next we have

$$
\begin{aligned}
& \int_{K_{m-1}}^{K_{m}} U^{-1} f_{2}\left(\ln \frac{K_{i}}{U}\right) d U \\
& =\ln K_{m} f_{2}\left(\ln \frac{K_{i}}{K_{m}}\right)-\ln K_{m-1} f_{2}\left(\ln \frac{K_{i}}{K_{m-1}}\right)-\frac{1}{2}\left(\ln K_{m}\right)^{2} \operatorname{myint}\left(M, \ln \frac{K_{i}}{K_{m}}, Y\right) \\
& +\frac{1}{2}\left(\ln K_{m-1}\right)^{2} \operatorname{myint}\left(M, \ln \frac{K_{i}}{K_{m-1}}, Y\right) \\
& -\frac{1}{2}\left(\ln K_{i}\right)^{2}\left[\operatorname{myint}\left(M, \ln \frac{K_{i}}{K_{m-1}}, Y\right)-\operatorname{myint}\left(M, \ln \frac{K_{i}}{K_{m}}, Y\right)\right] \\
& +\ln K_{i}\left[\operatorname{myint}\left(M, \ln \frac{K_{i}}{K_{m-1}}, Y-1\right)-\operatorname{myint}\left(M, \ln \frac{K_{i}}{K_{m}}, Y-1\right)\right] \\
& -\frac{1}{2}\left[\operatorname{myint}\left(M, \ln \frac{K_{i}}{K_{m-1}}, Y-2\right)-\operatorname{myint}\left(M, \ln \frac{K_{i}}{K_{m}}, Y-2\right)\right] \\
& =\ln K_{m} f_{2}\left(\ln \frac{K_{i}}{K_{m}}\right)-\ln K_{m-1} f_{2}\left(\ln \frac{K_{i}}{K_{m-1}}\right) \\
& +\frac{1}{2}\left[\left(\ln K_{i}\right)^{2}-\left(\ln K_{m}\right)^{2}\right] \operatorname{myint}\left(M, \ln \frac{K_{i}}{K_{m}}, Y\right) \\
& +\frac{1}{2}\left[\left(\ln K_{m-1}\right)^{2}-\left(\ln K_{i}\right)^{2}\right] \operatorname{myint}\left(M, \ln \frac{K_{i}}{K_{m-1}}, Y\right) \\
& +\ln K_{i}\left[\operatorname{myint}\left(M, \ln \frac{K_{i}}{K_{m-1}}, Y-1\right)-\operatorname{myint}\left(M, \ln \frac{K_{i}}{K_{m}}, Y-1\right)\right] \\
& -\frac{1}{2}\left[\operatorname{myint}\left(M, \ln \frac{K_{i}}{K_{m-1}}, Y-2\right)-\operatorname{myint}\left(M, \ln \frac{K_{i}}{K_{m}}, Y-2\right)\right] \text {, }
\end{aligned}
$$




$$
\begin{aligned}
& \int_{K_{i}}^{K_{N+1}} d U \int_{K_{m-1}}^{K_{m}} W^{-1} f_{2}\left(\ln \frac{U}{W}\right) d W \\
\ln \underline{\underline{\underline{W}}}=t & \int_{K_{m-1}}^{K_{m}} d W \int_{\ln \frac{K_{i}}{W}}^{\ln \frac{K_{N+1}}{W}} f_{2}(t) e^{t} d t \\
= & K_{m} \int_{\ln \frac{K_{i}}{K_{m}}}^{\ln \frac{K_{N+1}}{K_{m}}} f_{2}(t) e^{t} d t-K_{m-1} \int_{\ln \frac{K_{i}}{K_{m-1}}}^{\ln \frac{K_{N+1}}{K_{m-1}}} f_{2}(t) e^{t} d t \\
& -\int_{K_{m-1}}^{K_{m}} W f_{2}\left(\ln \frac{K_{i}}{W}\right) \frac{K_{i}}{W} \frac{W}{K_{i}} \frac{K_{i}}{W^{2}} d W \\
& +\int_{K_{m-1}}^{K_{m}} W f_{2}\left(\ln \frac{K_{N+1}}{W}\right) \frac{K_{N+1}}{W} \frac{W}{K_{N+1}} \frac{K_{N+1}}{W^{2}} d W \\
& K_{m} \int_{\ln \frac{K_{i}}{K_{m}}}^{\ln \frac{K_{N+1}}{K_{m}}} f_{2}(t) e^{t} d t-K_{m-1} \int_{\ln \frac{K_{i}}{K_{m-1}}}^{\ln \frac{K_{N+1}}{K_{m-1}}} f_{2}(t) e^{t} d t \\
& -K_{i} \int_{K_{m-1}}^{K_{m}} W^{-1} f_{2}\left(\ln \frac{K_{i}}{W}\right) d W+K_{N+1} \int_{K_{m-1}}^{K_{m}} W^{-1} f_{2}\left(\ln \frac{K_{N+1}}{W}\right) d W
\end{aligned}
$$

and

$$
\begin{aligned}
& \int_{K_{1}}^{K_{i}} d U \int_{K_{m}}^{K_{m+1}} W^{-1} f_{1}\left(\ln \frac{U}{W}\right) d W \\
\ln \frac{U}{\underline{\underline{W}}=t} & \int_{K_{m}}^{K_{m+1}} d W \int_{\ln \frac{K_{1}}{W}}^{\ln \frac{K_{i}}{W}} f_{1}(t) e^{t} d t \\
=\quad & K_{m+1} \int_{\ln \frac{K_{1}}{K_{m+1}}}^{\ln \frac{K_{i}}{K_{m}+1}} f_{1}(t) e^{t} d t-K_{m} \int_{\ln \frac{K_{1}}{K_{m}}}^{\ln \frac{K_{i}}{K_{m}}} f_{1}(t) e^{t} d t \\
& +\int_{K_{m}}^{K_{m+1}} W f_{1}\left(\ln \frac{K_{i}}{W}\right) \frac{K_{i}}{W} \frac{W}{K_{i}} \frac{K_{i}}{W^{2}} d W \\
& -\int_{K_{m}}^{K_{m+1}} W f_{1}\left(\ln \frac{K_{1}}{W}\right) \frac{K_{1}}{W} \frac{W}{K_{1}} \frac{K_{i}}{W^{2}} d W \\
= & K_{m+1} \int_{\ln \frac{K_{1}}{K_{m+1}}}^{\ln \frac{K_{i}}{K_{m}}} f_{1}(t) e^{t} d t-K_{m} \int_{\ln \frac{K_{1}}{K_{m}}}^{\ln \frac{K_{i}}{K_{m}}} f_{1}(t) e^{t} d t \\
& +K_{i} \int_{K_{m}}^{K_{m+1}} W^{-1} f_{1}\left(\ln \frac{K_{i}}{W}\right) d W-K_{1} \int_{K_{m}}^{K_{m+1}} W^{-1} f_{1}\left(\ln \frac{K_{1}}{W}\right) d W .
\end{aligned}
$$


Putting them together, we have

$$
\begin{aligned}
I & =\sum_{m=2}^{i-1}\left[(2-\Delta k) c_{m+1, j}-4 c_{m, j}+(2+\Delta k) c_{m-1, j}\right]\{ \\
& K_{N+1}\left(1+\ln K_{m}\right) f_{2}\left(\ln \frac{K_{N+1}}{K_{m}}\right)-K_{N+1}\left(1+\ln K_{m-1}\right) f_{2}\left(\ln \frac{K_{N+1}}{K_{m-1}}\right) \\
& +K_{i}\left[f_{2}\left(\ln \frac{K_{i}}{K_{m-1}}\right)-f_{2}\left(\ln \frac{K_{i}}{K_{m}}\right)\right] \\
& +K_{N+1}\left[1+\frac{1}{2}\left(\ln K_{N+1}\right)^{2}-\frac{1}{2}\left(\ln K_{m}\right)^{2}\right] \text { myint }\left(M, \ln \frac{K_{N+1}}{K_{m}}, Y\right) \\
& -K_{N+1}\left[1+\frac{1}{2}\left(\ln K_{N+1}\right)^{2}-\frac{1}{2}\left(\ln K_{m-1}\right)^{2}\right] \text { myint }\left(M, \ln \frac{K_{N+1}}{K_{m-1}}, Y\right) \\
& +K_{i}\left[\text { myint }\left(M, \ln \frac{K_{i}}{K_{m-1}}, Y\right)-m y i n t\left(M, \ln \frac{K_{i}}{K_{m}}, Y\right)\right] \\
& +K_{m}\left[\text { myint }\left(M-1, \ln \frac{K_{i}}{K_{m}}, Y\right)-m y i n t\left(M-1, \ln \frac{K_{N+1}}{K_{m}}, Y\right)\right] \\
& +K_{m-1}\left[\text { myint }\left(M-1, \ln \frac{K_{N+1}}{K_{m-1}}, Y\right)-m y i n t\left(M-1, \ln \frac{K_{i}}{K_{m-1}}, Y\right)\right] \\
& +K_{N+1} \ln K_{N+1}\left[m y i n t\left(M, \ln \frac{K_{N+1}}{K_{m-1}}, Y-1\right)-m y i n t\left(M, \ln \frac{K_{N+1}}{K_{m}}, Y-1\right)\right] \\
& \left.-\frac{1}{2} K_{N+1}\left[\operatorname{myint}\left(M, \ln \frac{K_{N+1}}{K_{m-1}}, Y-2\right)-\operatorname{myint}\left(M, \ln \frac{K_{N+1}}{K_{m}}, Y-2\right)\right]\right\}
\end{aligned}
$$


and

$$
\begin{aligned}
I I & =\sum_{m=i+1}^{N}\left[(2-\Delta k) c_{m+1, j}-4 c_{m, j}+(2+\Delta k) c_{m-1, j}\right]\left\{\begin{array}{l}
K_{1} \\
K_{m+1}
\end{array}\right. \\
& K_{1}\left(1+\ln K_{m+1}\right) f_{1}\left(\ln \left(1+\ln K_{m}\right) f_{1}\left(\ln \frac{K_{1}}{K_{m}}\right)\right. \\
& +K_{i}\left[f_{1}\left(\ln \frac{K_{i}}{K_{m}}\right)-f_{1}\left(\ln \frac{K_{i}}{K_{m+1}}\right)\right] \\
& +K_{1}\left[1+\frac{1}{2}\left(\ln K_{1}\right)^{2}-\frac{1}{2}\left(\ln K_{m}\right)^{2}\right] \text { myint }\left(G, \ln \frac{K_{m}}{K_{1}}, Y\right) \\
& -K_{1}\left[1+\frac{1}{2}\left(\ln K_{1}\right)^{2}-\frac{1}{2}\left(\ln K_{m+1}\right)^{2}\right] \text { myint }\left(G, \ln \frac{K_{m+1}}{K_{1}}, Y\right) \\
& +K_{i}\left[\text { myint }\left(G, \ln \frac{K_{m+1}}{K_{i}}, Y\right)-m y i n t\left(G, \ln \frac{K_{m}}{K_{i}}, Y\right)\right] \\
& +K_{m+1}\left[\operatorname{myint}\left(G+1, \ln \frac{K_{m+1}}{K_{1}}, Y\right)-m y i n t\left(G+1, \ln \frac{K_{m+1}}{K_{i}}, Y\right)\right] \\
& +K_{m}\left[\text { myint }\left(G+1, \ln \frac{K_{m}}{K_{i}}, Y\right)-m y i n t\left(G+1, \ln \frac{K_{m}}{K_{1}}, Y\right)\right] \\
& -K_{1} \ln K_{1}\left[\operatorname{myint}\left(G, \ln \frac{K_{m+1}}{K_{1}}, Y-1\right)-m y i n t\left(G, \ln \frac{K_{m}}{K_{1}}, Y-1\right)\right] \\
& \left.-\frac{1}{2} K_{1}\left[\text { myint }\left(G, \ln \frac{K_{m+1}}{K_{1}}, Y-2\right)-\text { myint }\left(G, \ln \frac{K_{m}}{K_{1}}, Y-2\right)\right]\right\}
\end{aligned}
$$




\section{BIBLIOGRAPHY}

[1] Leif Andersen and Jesper Andersen. Jump-diffusion processes: Volatility smile fitting and numerical methods for option pricing. Rev. Derivatives Research, 4:231-262, 2000.

[2] Donald W. K. Andrews and J. Christopher Monahan. An improved heteroskedasticity and autocorrelation consistent covariance matrix estimator. Econometrica, 60:953-966, 1992.

[3] David Applebaum. Lévy Processes and Stochastic Calculus. Cambridge University Press, 2004.

[4] L. Bachelier. Théorie de la spéculation. Annales Scientifiques L'École Normale Supérieure, 17:21-86, 1900.

[5] N. H. Bingham. Fluctuation theory in continutous time. Adv. Appl. Probab., 7:705-766, 1975.

[6] N. H. Bingham, C. M. Goldie, and J. L. Teugels. Regular Variation. Cambridge University Press, 1987.

[7] N. H. Bingham and R. Kiesel. Risk-Neutral Valuation. Pricing and Hedging of Financial Derivatives. Springer Finance, London, 1998.

[8] F. Black. Studies of stock price volatility changes. Proceedings of the Business and Economic Statistic Section, pages 177-181, 1976. 
[9] F. Black and M. Scholes. The pricing of options and corporate liabilities. Journal of Political Economy, 81:637-654, 1973.

[10] J. Y. Campbell and A. S. Kyle. Smart money, noise trading and stock price behaviour. Rev. Economic Studies, 60:1-34, 1993.

[11] Peter Carr, Hélyette Geman, Dilip B. Madan, and Marc Yor. The fine structure of asset returns: An empirical investigation. Journal of Business, 75, 2002.

[12] Peter Carr, Hélyette Geman, Dilip B. Madan, and Marc Yor. Leveraging lévy via lamperti. preprint notes, 2003.

[13] Peter Carr, Hélyette Geman, Dilip B. Madan, and Marc Yor. From local volatility to local lévy models. Soumis Quantitative Finance, 2004.

[14] Peter Carr and Dilip B. Madan. Option valuation using the fast fourier transform. Journal of Computational Finance, 1999.

[15] T. Chan. Pricing contigent claims on stocks driven by lévy processes. Ann. Appl. Probab., 9:504-528, 1999.

[16] T. H. F. Cheuk and T. C. F. Vorst. Complex barrier options. Journal of Derivatives, 4:8-22, 1996.

[17] R. Cont and P. Tankov. Financial Modelling with Jump Processes. Chapman \& Hall CRC Press, 2003. 
[18] Rama Cont and Ekaterina Voltchkova. Intergro-differential equations for option prices in exponential lévy models. Ecole Polytechnique, Centre De Mathématiques Appliquées, R.I. No. 547, 2004.

[19] J. C. Cox. The constant elasticity of variance option pricing model. The Journal of Portfolio Management, pages 15-17, 1996.

[20] J. C. Cox, J. E. Jr. Ingersoll, and S. A. Ross. A theory of the term structure of interest rates. Econometrica, 53(2), 1985.

[21] E. B. Davies. One-Parameter Semigroups,. Academic Press, 1980.

[22] Freddy Delbaen and Hiroshi Shirakawa. A note of option pricing for constant elasticity of variance model. Asia-Pacific Financial Markets, 2002.

[23] Emanuel Derman and Iraj Kani. Riding on a simile. Risk, 7(2):32-39, 1994.

[24] Y. D'Halluin, P. Forsyth, and G. Labahn. A penalty method for american options with jump-diffusion processes. Working Paper, University of Waterloo, 2003.

[25] Bruno Dupire. Pricing with a smile. Risk, 7(1):18-20, 1994.

[26] E. B. Dynkin. Markov Processes I. Berlin-Heidelber-New York: Springer, 1965.

[27] B. E. Fristedt. Sample functions of stochastic processes with stationary, independent increments. Advances in Probability, 3:241-396, 1974.

[28] Chi fu Huang and Robert H. Litzenberger. Foundations for Financial Economics. Elsevir Science Pub. Co., 1988. 
[29] A. Ronald Gallant. Nonlinear Statistical Models. John Wiley \& Sons: New York, 1987.

[30] I. Gihman and A. V. Skorohod. The Theory of Stochastic Processes II. Springer, Berlin, 1975.

[31] L. R. Glosten, R. Jagannathan, and D. Runkle. Relationship between the expected value and the volatility of the nominal excess return on sotcks. Journal of Finance, 48:1779-1802, 1993.

[32] Alastair R. Hall. Generalized Method of Moments. Oxford University Press, 2005.

[33] Lars Peter Hansen. Large sample properties of generalized method of moments estimators. Econometrica, 50:1029-1054, 1982.

[34] Lars Peter Hansen and Robert J. Hodrick. Forward exchange rates as optimal predictions of future spot rates: An empirical analysis. Journal of Political Economy, 88:829-853, 1980.

[35] Lars Peter Hansen and Kenneth J. Singleton. Generalized instrumental variables estimation of nonlinear rational expectations models. Econometrica, 50:1269-1286, 1982.

[36] S. Heston and G. Zhou. On the rate of convergence of discrete-time contingent claims. Mathematical FInance, 10:53-75, 2000. 
[37] Ali Hirsa and Dilip B. Madan. Pricing american options under variance gamma. Journal of Computational Finance, 7, 2003.

[38] K. Itô. Lectures on Stochastic Processes. Tata Institute of Fundamental Research. Springer, Berlin, 1961.

[39] Martin Jacobsen and Marc Yor. Multi-self-similar markov processes on $\mathbb{R}_{+}^{n}$ and thier lamperti representations. Probability Theory and Related Fields, 2003.

[40] J. Jacod. Calcul Stochastique et Problémes de Martingales. Lecture Notes in Math. 714. Springer, Berlin, 1979.

[41] J. Jacod and A. N. Shiryaev. Limit Theorems for Stochastic Processes. Springer, Berlin, 1987.

[42] Robert Jarrow and Stuart Turnbull. Derivatives Securities. South-Western College Publishing, 1996.

[43] Jr. Jonathan E. Ingersoll. Theory of Financial Decision Making. Rowman \& Littlefield Publishers, Inc., 1987.

[44] John Lamperti. Semi-stable stochastic processes. Transactions of the American Mathematical Society, 104(1):62-78, July 1962.

[45] John Lamperti. Semi-stable markov processes. i. Z. Wahrscheinlichkeitstheorie und Verw. Gebiete, 1972.

[46] P. Lévy. Theorie de l'addition des variables aleatoires. Gauthier-Villers, Paris, 1937. 
[47] P. Lévy. Théorie de l'addition des variables aléatoires. 2nd edn. GauthierVillars, Paris, 1954.

[48] M. Loeve. Probability Theory, Sec. ed. Princeton: Van Nostrand, 1960.

[49] B. Mandelbrot. The variation of certain speculative prices. The Journal of Business, 36:394-419, 1963.

[50] A. M. Matache, T. Von Petersdorff, and C. Schwab. Fast deterministic pricing of options on lévy driven assets. M2AN Math. Model. Numer. Anal, 38:37-71, 2004.

[51] R. C. Merton. Theory of rational option pricing. Bell Journal of Economics and Management Science, 4:141-183, 1973.

[52] D. B. Nelson. Conditional heteroskedasticity in asset pricing: a new approach. Econometrica, 59:347-370, 1991.

[53] Whitney Newey and Kenneth West. A simple positive semi-definite, heteroskedasticity and autocorrelation consistent covariance matrix. Econometrica, 55:703-708, 1987.

[54] M. F. M. Osborne. Brownian motion in the stock market. Operation Research, 7:145-173, 1959.

[55] D. M. Pooley, K. R. Vetzal, and P. A. Forsyth. Convergence remedies for non-smooth payoffs in option pricing. Journal of Computational Finance, 6, 2003. 
[56] S. J. Press. A compound events model for security prices. Journal of Business, 40:317-335, 1967.

[57] P. Protter. Stochastic Integration and Differential Equations: A New Approach. Springer Berlin, 1990.

[58] L. C. G. Rogers and D. Williams. Diffusions, Markov Processes, and Martingales vol. I: Foundations. Wiley, New York, 1994.

[59] P. Samuelson. Rational theory of warrant pricing. Industrial Management Review, 6:13-32, 1965.

[60] K. Sato. Stochastic Processes with Stationary Independent Increments (in Japanese). Kinokuniya, Tokyo, 1990.

[61] K. Sato. Lévy Processes on the Euclidean Spaces. University of Zurich, 1995.

[62] Wim Schoutens. Lévy Processes in Finance, Pricing Financial Derivatives. John Wiley \& Sons Ltd., 1999.

[63] T. Shiga and S. Watanabe. Bessel diffusions as a one-parameter family of diffusion processes. Z. Wahrscheinlichkeitstheorie, 27:37-46, 1973.

[64] A. V. Skorohod. Random Processes with Independent Increments. Kluwer, Dordrecht, Netherlands, 1991.

[65] D. Tavella and C. Randall. Pricing Financial Instruments: The Finite Difference Method. Wiley, New York, 2000. 
[66] S. J. Taylor. Sample path properties of processes with stationary independent increments. Stochastic Analysis. Wiley London, pages 387-414, 1973.

[67] V. Thomée and L. B. Wahlbin. Convergence rates of parabolic difference schemes for non-smooth data. Mathematics of Computation, 28(125):1-13, 1974.

[68] L. B. Wahlbin. A remark on parabolic smoothing and the finite element method. SIAM Journal on Numerical Analysis, 17(1):33-38, 1980.

[69] Halbert White. A heteroskedasticity-consistent covariance matrix estimator and a direct test for heteroskedasticity. Econometrica, 48:817-838, 1980.

[70] D. Williams. Path decomposition and continuity of local time for onedimensional diffusions. Proc. London Math. Soc, 28:738-768, 1974.

[71] M. Yor, P. Carmona, and F. Petit. On exponential functionals of certain lévy processes. Stochastics and Stochastic Report, 47:71-101, 1994.

[72] Marc Yor. Exponential Functionals of Brownian Motion and Related Processes. Springer-Verlag Berlin Heidelber New York, 2001. 